\title{
Supersymmetric Versions of the Standard Model
}

\author{
André Luiz Carvalho de Gouvêa \\ Ph.D. Thesis \\ Department of Physics \\ University of California, Berkeley \\ and \\ Physics Division \\ Ernest Orlando Lawrence Berkeley National Laboratory \\ University of California \\ Berkeley, CA 94720
}

May 1999

This work was supported by the Director, Office of Energy Research, Office of High Energy and Nuclear Physics, Division of High Energy Physics, of the U.S. Department of Energy under Contract No. DE-AC0376 SF00098. 


\section{DISCLAIMER}

This report was prepared as an account of work sponsored by an agency of the United States Government. Neither the United States Government nor any agency thereof, nor any of their employees, make any warranty, express or implied, or assumes any legal liability or responsibility for the accuracy, completeness, or usefulness of any information, apparatus, product, or process disclosed, or represents that its use would not infringe privately owned rights. Reference herein to any specific commercial product, process, or service by trade name, trademark, manufacturer, or otherwise does not necessarily constitute or imply its endorsement, recommendation, or favoring by the United States Government or any agency thereof. The views and opinions of authors expressed herein do not necessarily state or reflect those of the United States Government or any agency thereof. 


\section{DISCLAIMER}

Portions of this document may be illegible in electronic image products. Images are produced from the best available original document. 
André Luiz Carvalho de Gouvêa

B.A. (Pontifícia Universidade Católica do Rio de Janeiro, Brazil) 1994

A dissertation submitted in partial satisfaction of the

requirements for the degree of

Doctor of Philosophy

in

Physics

in the

GRADUATE DIVISION

of the

UNIVERSITY of CALIFORNIA, BERKELEY

Committee in charge:

Professor Hitoshi Murayama, Chair

Professor Lawrence Hall

Professor Nicolai Reshetikhin

Spring 1999 


\section{Supersymmetric Versions of the Standard Model}

Copyright $\odot 1999$

by

André Luiz Carvalho de Gouvêa

The U.S. Department of Energy has the right to use this document for any purpose whatsoever including the right to reproduce all or any part thereof. 


\begin{abstract}
SUPERSYMMETRIC VERSIONS OF THE STANDARD MODEL

by
\end{abstract}

André Luiz Carvalho de Gouvêa

Doctor of Philosophy in Physics

University of California, Berkeley

Professor Hitoshi Murayama, Chair

The Standard Model of particle physics is one of the most successful models in physics. With only a handful of parameters, it is capable of predicting/explaining the results of all high energy physics experiments, with astounding precision. The Standard Model, however, is by no means free of problems.

The Standard Model, which is a quantum field theory, must be an effective description of some other, unknown, theory. This is known because of ultraviolet divergences, which have to be regularized and renormalized. Furthermore, because of the so-called hierarchy problem, there must be new, beyond the Standard Model, physics at or slightly below the $\mathrm{TeV}$ energy scale, which is within the reach of the next generation of accelerator experiments.

Low-energy supersymmetry is one of the most popular solutions to the hierarchy problem. It is, therefore, important to try and predict what experimental 
signals one should look for if Nature is supersymmetric at the $\mathrm{TeV}$ scale.

This dissertation addresses some of the possible manifestations of supersymmetric versions of the Standard Model. Most of the issues will concern the socalled Minimal Supersymmetric Standard Model (MSSM), where the Standard Model Lagrangian is supersymmetrized and the smallest allowed number of extra fields/parameters is added. The parameter space of the MSSM can already be constrained by current experiments. One particular method for constraining the gluino (supersymmetric partner of the gluon) mass will be studied, where one uses the existing LEP 4-jet data to rule out the existence of light gluinos.

The minimal parameterizations of the MSSM (in particular of the supersymmetry breaking parts of the MSSM) will be presented and criticized, and a different (less minimal) alternative will be suggested and analyzed. Some attention will be given to models with the gauge mediation of supersymmetry breaking, in particular the cosmology of such models will be studied. The $\mu$-problem in such models is also discussed in detail, and the Next-to-Minimal Supersymmetric Standard Model is presented and studied as a solution. 
20. 


\section{Contents}

1 Introduction 1

2 The Minimal Supersymmetric Standard Model $\quad 6$

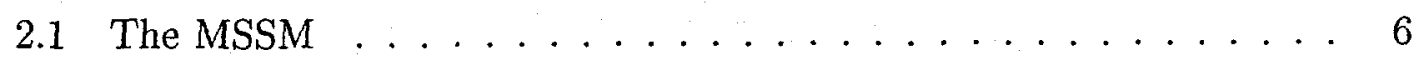

2.2 What is known about the MSSM ........... 16

2.3 Excluding Light Gluinos from Z Decays . . . . . . . . . . . . . 22

3 Parameterizing the MSSM 39

3.1 Introduction . . . . . . . . . . . . . 39

3.2 The Standard Parameterizations . . . . . . . . . . . 40

3.3 Consequences of the Minimal Parameterization . . . . . . . . . 43

3.4 A Less Constrained Parameterization . . . . . . . . . . . . 46

3.5 Some Phenomenological Consequences ... . . . . . . 50

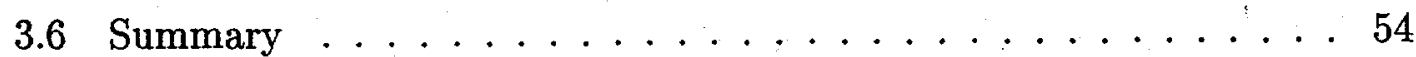

4 Cosmology of Models with Gauge Mediated SUSY Breaking 55

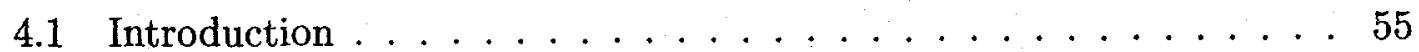

4.2 Cosmology of a Light Gravitino . . . . . . . . . . 57

4.3 Light Gravitino in the LEGM Models . . . . . . . . . . . . 61

4.4 Flat Directions in the LEGM Models . . . . . . . . . . . 71

4.5 Affleck-Dine Baryogenesis in the LEGM Models . . . . . . . . . . 74 
4.6 Cosmology of String Moduli _ . . . . . . . . . . . 990

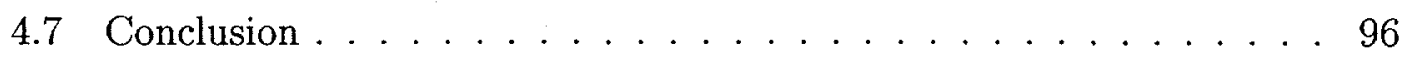

5 The $\mu$-Problem and the Next-to-Minimal SSM 98

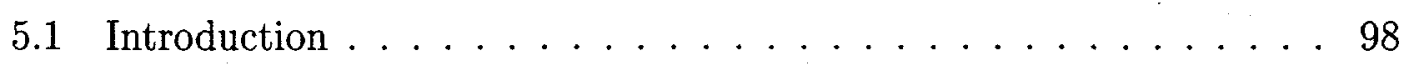

5.2 The $\mu$-problem in the GMSB . . . . . . . . . . 101

5.3 The $\mu$-parameter in the MSSM $\ldots \ldots \ldots \ldots \ldots \ldots$

5.4 The NMSSM with the GMSB $\ldots \ldots \ldots \ldots \ldots \ldots$

5.5 Possible Modifications to the NMSSM . . . . . . . . . 139

5.6 Conclusion . . . . . . . . . . . . . . . 148

6 Conclusions 151

$\begin{array}{ll}\text { A Computing the Effective Potential } & 156\end{array}$

B Effective Potential and Wave-function Renormalization 165

C Time Evolution of the Flat Direction $\quad 167$

D Estimation of the Primordial Baryon Asymmetry 169

E Affleck-Dine Baryogenesis in Hidden Sector Scenarios $\quad 171$

F The Renormalization Group Equations of the (N)MSSM $\quad 176$

$\begin{array}{ll}\text { G Scalar Higgs Mass-Squared Matrix } & 180\end{array}$

$\begin{array}{lc}\text { H Comments on Naturalness } & 182\end{array}$ 
I The Dependence of the Higgs VEVs on the couplings of the modified NMSSM 


\section{List of Figures}

2.1 One of the many proton decay diagrams which arise in the MSSM in the absence of R-parity. . . . . . . . . . . . . 11

2.2 One of the SUSY contributions to $\mu^{-} \rightarrow e^{-} \gamma$. The cross represents the insertion of the $\left(m_{\tilde{e}}^{2}\right)^{12}$ off-diagonal slepton mass-squared parameter (see Eq. $(2.1 .14)) \ldots \ldots \ldots \ldots \ldots \ldots \ldots$

2.3 One of the SUSY contributions to $K^{0} \leftrightarrow \bar{K}^{0}$ mixing. $\tilde{q}$ represents any of the squarks. Here, the flavor-changing effects have been rotated to the $\tilde{g} \tilde{q} q^{\prime}$ couplings. ..................... 18

2.4 Extracted QCD color factors from the OPAL analysis [29]. The shown $\chi^{2}$ values correspond to $39.3 \%, 68 \%$ and $95 \%$ confidence levels with two degrees of freedom. The constraint $C_{A} / C_{F}=9 / 4$ is imposed (vertical solid line) and one is limited to the unshaded region $\left(T_{F} / C_{F} \geq 3 / 8\right)$ in order to put constraints on a possible light gluino contribution to the four-jet events from $Z$ decays. See the text for more details. . . . . . . 29

2.5 Effective contribution to $T_{F} / C_{F}$ of a massive secondary quark relative to the massless case. The solid line shows the reduction in the rate alone. The other two lines include the effect that the distributions in $\mathrm{BZ}$ and NR angles change due to finite quark mass. $y_{\text {cut }}=0.03$ and $\sqrt{s}=m_{Z} c^{2}=91.17 \mathrm{GeV}$ was assumed 
2.6 The distributions in BZ and NR angles of the $q \bar{q} q \bar{q}$ final state where the secondary quark has a mass of $5 \mathrm{GeV} / c^{2}$. They can be fit extremely well as a linear combination of massless $q \bar{q} q \bar{q}$ and $q \bar{q} g g$ distributions.

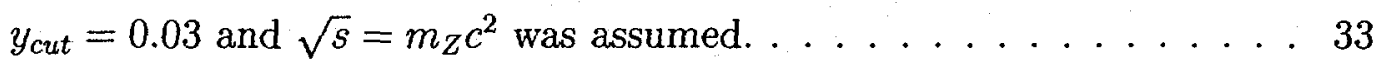

2.7 Exclusion confidence level of a light gluino as a function of its mass. Two curves are shown depending on the method of estimating the finite mass effects. In either case, a light gluino of mass below $1.5 \mathrm{GeV} / c^{2}$ is excluded at more than $90 \%$ confidence level. . . . . . . . . 34

3.1 Parameter space analysis indicating the nature of the LSP. The solid line indicates the points allowed by the VMSSM and the dashed line represents points where the gaugino content of $\tilde{\chi}_{1}^{0}$ is $50 \% . A_{0}=0$, $m_{0}^{2}=500^{2}(\mathrm{GeV})^{2}$ and $\tan \beta=10$. The bounds $m_{A}>65 \mathrm{GeV}$, $m_{\tilde{\nu}}>43 \mathrm{GeV}, m_{\tilde{\tau}}>67 \mathrm{GeV}$ (if $m_{\tilde{\tau}}<m_{\tilde{\chi}_{1}^{0}}$ ), and $m_{\tilde{\chi}_{1}^{ \pm}}>65 \mathrm{GeV}$ were imposed. . . . . . . . . . . . . . . 49

3.2 Same as Fig. 3.1, for $A_{0}=0, m_{0}^{2}=700^{2}(\mathrm{GeV})^{2}$ and $\tan \beta=2 \ldots \ldots 50$ 
4.1 The upper bound on $T_{\max }$ as a function of the gravitino mass from the requirement that the relic stable gravitinos do not overclose the Universe. The Hubble parameter is taken to be $H_{0}=100 \mathrm{Mpc} / \mathrm{km} / \mathrm{sec}$. There is no constraint below $m_{3 / 2}=2 \mathrm{keV}$, which is represented by the vertical line. For smaller $H_{0}$, the constraints become more stringent. The upper bound on $T_{\max }$ shifts towards smaller $T_{\max }$ as $\left(H_{0}\right)^{2}$. The vertical line moves towards smaller $m_{3 / 2}$ also as $\left(H_{0}\right)^{2}$. Note that the current data prefer $H_{0} \sim 70 \mathrm{Mpc} / \mathrm{km} / \mathrm{sec} . \ldots \ldots \ldots 62$

4.2 The initial motion of the flat direction with the potential given in Eq. (4.5.22). Here, $m_{3 / 2}=100 \mathrm{keV}$, and $\phi_{0}=0.2 M_{*} e^{i \pi / 8}$ was taken. . . . 84

4.3 The resulting baryon-to-entropy ratio as a function of the initial amplitude $\phi_{0}$. The parameters are taken to be $V_{0}=\left(3 \times 10^{3} \mathrm{GeV}\right)^{4}, \theta_{0}=\pi / 8$, $\phi_{\mathrm{dec}}=10^{5} \mathrm{GeV}$, and $m_{3 / 2}=1 \mathrm{keV}$ (dotted line), $m_{3 / 2}=100 \mathrm{keV}$ (solid

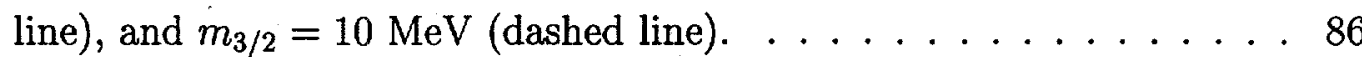

5.1 Lower bounds on $\mu$ in models with the GMSB subject to the constraint $M_{Z}=91 \mathrm{GeV}$ and to the lower bounds on superparticle masses (see text), (a) as a function of the messenger scale, for $\tan \beta=2,10$, and 30 , and (b) as a function of $\tan \beta$ for a fixed messenger scale of $10^{8} \mathrm{GeV} .120$ 
5.2 (a) Lower bounds on $\left|m_{H_{u}}^{2}\right|^{1 / 2}$ and $\left|m_{H_{d}}^{2}\right|^{1 / 2}$ as a function of the messenger scale $\Lambda$ from the selectron mass constraint $m_{\tilde{e}}>80 \mathrm{GeV}$. Here $n=1, h_{t}=1.07, k=0.3$ and $\lambda=0.29$ at the weak scale. These bounds do not change for different values of $k$ or $\lambda$. The other plots show typical values of (b) $A_{\lambda}$, (c) $A_{k}$, and (d) $m_{N}^{2}$, for the same choice of parameters that yielded (a). The values of these parameters do not change significantly for different values of $k$ or $\lambda$.

5.3 The value of $v \equiv \sqrt{v_{d}^{2}+v_{u}^{2}}$ as a function of $\lambda$ and $k$. The inputs are $n=1, m_{N}^{2}=-(190 \mathrm{GeV})^{2}, B=50 \mathrm{TeV}, \Lambda=100 \mathrm{TeV}, h_{t}=0.99 \ldots 142$

5.4 The dependence of $v$ on the value of $\lambda$ for the high- and low-energy GMSB. The other input parameters are the same as in Fig. 5.3. . . . 142

A.1 Feynman diagrams which contribute to the vacuum energy in the background of the flat direction $\phi=\bar{\phi}$. The vertices are due to the $D$-term potential. The scalar field with mass $m=2 g\langle\phi\rangle$ is the scalar component of the massive gauge multiplet in the presence of the background $\phi$. The scalar fields with masses $M_{+}$and $M_{-}$are the messenger scalars. 157

A.2 A Feynman diagram with the gaugino of mass $m$, the messenger fermion of mass $M$, and the messenger scalars of mass $M_{ \pm} \ldots \ldots \ldots 157$

A.3 Feynman diagrams with vacuum polarization due to (C1) messenger scalar loops, (C2) "seagull" diagram with messenger scalars, and (C3) messenger fermions. . . . . . . . . . . . . . . . . 158 
A.4 A plot of the effective potential Eq. (A.15) for $z \equiv 2 g|\phi| / M<30$, in the unit of $g^{2}(M B)^{2} /\left(128 \pi^{4}\right)$. The solid line is the exact result, and the dotted line shows the asymptotic form Eq. (A.16) valid for large $z .162$

A.5 A plot of the effective potential Eq. (A.15) (or equivalently, Eq. (A.19)) for the small field amplitude, $z \equiv 2 g|\phi| / M<1$, in the unit of $g^{2}(M B)^{2} /\left(128 \pi^{4}\right)$. The solid line is the exact result, and the dotted line shows the approximate form Eq. (A.20) valid for small $z$. . . . 164

H.1 The probability densities of finding specific values of $v$ in the NMSSM with extra vector-like quarks upon random choices of $\lambda$. All other parameters are the same as in Fig. 5.4. The probability densities are normalized so that $P(v=174 \mathrm{GeV})=1 \ldots \ldots \ldots \ldots$ 


\section{List of Tables}

3.1 SUSY-breaking parameters at a scale of $500 \mathrm{GeV}$ from the 1-loop RG equations with the VMSSM boundary conditions at $M_{G U T}=1.86 \times$ $10^{16} \mathrm{GeV}$; for (A) the first/second generation sfermions and (B) the rest with $\tan \beta=10$. The masses of first/second generation fermions have been neglected, and $h_{t}\left(m_{t}\right)=165 /(174 \sin \beta)$ was used. The table is to be read as follows: each soft parameter is a linear combination of the input parameters, with the coefficients given in the table. For example, $m_{H_{d}}^{2}=0.95 m_{0}^{2}+0.38\left(M_{1 / 2}\right)^{2}-0.01\left(A_{0}\right)^{2}-0.04 M_{1 / 2} A_{0}-1 / 2 D_{Y}$ and $A_{\tilde{d}}=A_{0}+3.41 M_{1 / 2} \ldots \ldots \ldots \ldots \ldots$

5.1 The numerically determined NMSSM parameters for five sample points in the parameter space. Here $m_{h_{i}}$ and $m_{A_{i}}$ refer to the eigenvalues of the scalar and pseudoscalar Higgs mass matrices respectively, and $m_{\tilde{e}}$ denotes the mass of the right-handed selectron. The values of $\lambda, k$, and $h_{t}$ are given at the weak scale. All the other quantities have been defined earlier in the text. .................. 131 


\section{Acknowledgements}

It is with great pleasure that I thank my advisor, Hitoshi Murayama, for his wisdom, his patience, his eternal good mood, and for coming up with the most informative and interesting research topics. Were it not for him, none of the work done here would have happened. I thank Alex Friedland for collaborating in most of the topics included in this dissertation, and also for many insightful discussions and nice talks. I also thank Kaustubh Agashe and Michael Graesser, my office mates, Dave Smith and Neal Weiner for many interesting discussions, Takeo Moroi for his collaboration in part of this thesis, and the whole theory group at LBNL for making my experience here pleasant and incredibly fruitful. In particular I thank Professors Mahiko Suzuki, Lawrence Hall, and Hiroshi Ooguri for teaching me some very important physics during courses at UC Berkeley. I would also like to acknowledge Bob Jacobsen for his orientation during my first year at Berkeley and Marjori Shapiro and Nikolai Reshetikhin for agreeing to participate in my qualifying exam and/or dissertation committee. I am grateful to Anne Takizawa and Donna Sakima of the Physics Department and Barbara Gordon and Mary Kihanya at LBNL for help with administrative work. I thank CNPq (Brazil) for their financial support. I am indebted to all of the friends I was lucky enough to make during my stay at Berkeley, and some of the old ones, for making the last four years outstanding. Finally, I thank my wife, Marcia, for her love and unconditional support and my daughter, Andréa. 


\section{Chapter 1}

\section{Introduction}

The Standard Model (SM) of particle physics [1] is one of the most successful models in the entire history of physics. It contains only a handful of fundamental fields (quarks, leptons, gauge bosons, the Higgs boson) and free parameters (gauge and Yukawa couplings, Higgs self-coupling and Higgs mass-squared) and is capable of explaining all experimental particle physics results.* Some physical quantities, such as the anomalous magnetic moments of the muon and the electron [3] have been calculate and measured with unprecedented accuracy, and the agreement between theory and experiment is astounding.

The SM is a quantum field theory (more specifically a gauge theory), and, as such, is plagued with ultraviolet divergences. These divergences can be properly regularized and renormalized away, such that, in the end, one has a finite and predictive theory. From a more fundamental point of view, one is led to describe the SM as a "low" energy effective theory of some, yet unknown, more fundamental theory. From this perspective, the need for regularizing/renormalizing the SM is simply an indication that the $\mathrm{SM}$ is not appropriate for describing physical processes which involve energy scales higher than some unknown cut-off. It is

*Recently there has been evidence of physics beyond the SM in the neutrino sector [2]. The new experimental data can be explained, however, if one adds neutrino masses to the SM. 
important to remember that the dependence of physical quantities on the cut-off energy scale is removed by allowing the physical parameters (masses and coupling constants) to vary as a function of the probed energy scale (running parameters), via a renormalization group analisis [1].

If this modern understanding of the SM is correct, beyond the SM physics should be detected already at the multi-hundred GeV level. This is due to the "hierarchy problem", which is closely related to the presence of fundamental scalars in the SM, and is briefly discussed in the next paragraphs.

Quantum field theory is supposed to be valid up to the Planck Scale $\left(M_{P l}=\right.$ $\left.\left(G_{N} / \hbar c\right)^{-1 / 2} \approx 10^{19} \mathrm{GeV}\right)$, where gravitational effects become comparable to the SM gauge interactions and quantum field theory is necessarily inapplicable. In order to determine the range of validity for the Standard Model, however, one must be more careful.

In the presence of fundamental scalars, which is the case of the Standard Model Higgs field, one must worry about the following issue: the scalar Higgs potential is

$$
V(H)=\frac{m_{0}^{2}}{2}|H|^{2}+\frac{\lambda_{0}}{4}|H|^{4},
$$

where both $m_{0}^{2}$ and $\lambda_{0}$ are bare parameters, which are to be be renormalized. Quantum corrections to $m_{0}^{2}$ are quadratically divergent, and, after renormalization, the effective Higgs mass-squared parameter is $m_{s}^{2} \approx m_{0}^{2}+\Lambda^{2}$, where $\Lambda$ is the scale where the SM ceases to be an appropriate description for physics (cut-off). Numerically, electroweak symmetry breaking requires $m_{s}^{2} \sim-(100)^{2}(\mathrm{GeV})^{2}$. If 
$\Lambda \sim M_{P l}$, an incredible amount of fine tuning (1 part in $\left.10^{34}\right)$ is required in order to explain electroweak symmetry breaking. It is clear that a "natural" order of magnitude value for the Higgs mass-squared parameter is $\Lambda^{2}$. This is the hierarchy problem. It is a clash between the fact that quantum field theory should be a good language for describing physics up to the Planck Scale $\left(\Lambda \approx M_{P l}\right)$ and the fact that the Higgs mass-squared parameter, which should "naturally" be of order $\Lambda^{2}$, is forced, in order explain electroweak symmetry, to be 34 orders of magnitude smaller. Any satisfactory solution to this problems predicts that there is new physics at the several hundred GeV energy scale.

There are different types of solution to the hierarchy problem. Some imply that there are no fundamental scalars, and that the Higgs boson is in reality a composite field, made up of some (extra) fundamental fermions [4]. Another, very recent, solution [5] implies that quantum field theory actually breaks down at the $\mathrm{TeV}$ scale, where gravity becomes strong. In order to be phenomenologically viable, this solution requires that there are more, compactified, dimensions, in which the gravitional field propagates.

The other type of solution implies that there is some symmetry reason which forbids scalar mass-squared parameters from being renormalized by $\Lambda^{2}$. This is exactly what happens in the case of fermion masses. Chiral symmetry prevents massless fermions from acquiring a mass due to quantum effects and furthermore guarantees that fermion masses are only logarithmically renormalized, as $m_{f}=$ $m_{f, 0}(1+\log (\Lambda / \mu))$, where $m_{f, 0}$ is the bare fermion mass, $\mu$ is the renormalization 
scale and $\Lambda$ is the cut-off. Supersymmetry (SUSY) is a symmetry which transforms scalars into fermions (and vice-versa) [6], and therefore, because fermion masses only receive logarithmic corrections, so do the scalar mass-squared parameters.

Presently, despite a lot of experimental effort, there is no evidence for physics beyond the SM. There are many manifestations for new physics, and they fall into three "general" categories: (i) precision measurements, or measurements of very suppreseed quantities, such as anomalous magnetic moments, very rare decay processes (such as $K \rightarrow \pi \nu \bar{\nu}$ ), CP-violation, etc; (ii) violation of global (accidental within the SM) symmetries, such as baryon number (proton decay), lepton number $(\mu \rightarrow e \gamma)$, etc; (iii) direct detection of new "fundamental" degrees of freedom (in the case of SUSY, supersymmetric partners of the SM fields).

Given the situation of experimental particle physics today, unbiased/nonmotivated searches for new physics are impractical, if not impossible, and phenomenological models are fundamental. This dissertation discusses low energy SUSY as the solution to the hierarchy problem, in particular how supersymmetric versions of the SM are modeled and constrained.

This thesis is organized as follows: in Chap. 2 The minimal supersymmetric standard model (MSSM) is briefly introduced and discussed. In particular its parameter space will be introduced and its particle spectrum spelled out. Present experimental constraints on the MSSM are briefly discussed and one procedure for excluding light gluinos, which are particularly elusive, with LEP data is proposed and discussed. In Chap. 3 the need for properly parameterizing of the MSSM, 
in particular its SUSY breaking sector, is addressed, and a brief review of the "standard" paraterizations is provided. These are analyzed and criticized, and an improved parameterization is proposed and discussed. In Chap. 4 the gauge mediation of SUSY breaking is addressed in some detail, and, in particular, the cosmology of such models is studied. In Chap. 5 the "problem of the $\mu$-parameter" is carefully introduced and studied in models with the gauge mediation of SUSY breaking. The Next-to-Minimal Supersymmetric SM is presented and studied as a possible solution. Chap. 6 contains a summary of the results obtained and some conclusions. 


\section{Chapter 2}

\section{The Minimal Supersymmetric Standard Model}

In this chapter, the Minimal Supersymmetric Standard Model (MSSM) is introduced. In the first section, its particle content at low energies (below the electroweak symmetry breaking scale) is described. In the second section, a brief discussion of what is known about the parameter space of the MSSM follows, and the third section discusses one particular way of searching for light gluinos.

\subsection{The MSSM}

The Minimal Supersymmetric Standard Model (MSSM) is the smallest (as far as the number of fundamental fields is concerned) extension of the Standard Model of particle physics (SM) which is supersymmetric. It contains all the SM fermion and gauge boson fields, plus their supersymmetric partners, the "sfermions," which are scalar fields, and the "gauginos," which are fermions. It also contains two scalar Higgs doublets, plus their fermionic superpartners, the "higgsinos" [7].

The SM Lagrangian consists of the most general renormalizable Lagrangian which is invariant under a specific gauge group, with matter fields which transform under certain representations of this gauge group.

The SM gauge group is the direct product of three simple compact groups, 
$S U(3)_{c}$, the color gauge group, which mediates strong interactions, $S U(2)_{L}$, and $U(1)_{Y}$, which are responsible for mediating the electroweak interactions. The subscripts $L$ and $Y$ stand for "left-handed" and "weak hypercharge" respectively. The electroweak part of the SM gauge group is spontaneously broken by a scalar field (the Higgs field) condensate, yielding the electromagnetic gauge interactions $\left(U(1)_{e m}\right)$ and the weak interactions, which are mediated by massive $W^{ \pm}, Z^{0}$ vector bosons.

The SM fermions can be described in terms of left-handed chiral fermions and their anti-particles. Explicitly they are, according to their gauge quantum numbers:

$$
\begin{array}{llll}
q_{i}= & \left(\begin{array}{c}
u \\
d
\end{array}\right)_{L}, & \left(\begin{array}{c}
c \\
s
\end{array}\right)_{L}, & \left(\begin{array}{c}
t \\
b
\end{array}\right)_{L}, \\
l_{i}= & \left(\begin{array}{c}
\nu_{e} \\
e
\end{array}\right)_{L}, & \left(\begin{array}{c}
\nu_{\mu} \\
\mu
\end{array}\right)_{L}, & \left(\begin{array}{c}
\nu_{\tau} \\
\tau
\end{array}\right)_{L}, \\
u_{i}= & \left(u^{c}\right)_{L}, & \left(c^{c}\right)_{L}, & \left(t^{c}\right)_{L}, \\
d_{i}= & \left(d^{c}\right)_{L}, & \left(s^{c}\right)_{L}, & \left(b^{c}\right)_{L}, \\
e_{i}= & \left(e^{c}\right)_{L}, & \left(\mu^{c}\right)_{L}, & \left(\tau^{c}\right)_{L} .
\end{array}
$$

The superscript $c$ stands for charge conjugate. $u_{1} \equiv\left(u^{c}\right)_{L}$ is therefore a lefthanded anti-up-quark. It is an $S U(2)_{L}$ singlet. Its anti-particle is the righthanded up-quark. This representation will prove to be particularly useful when one describes the MSSM Lagrangian.

Before writing the MSSM Lagrangian, it is useful to describe chiral and vector 
superfields (see [6]). Matter particles and their supersymmetric partners can be nicely accommodated into chiral superfields, which can be. written in terms of component fields as

$$
\Phi(y)=\phi(y)+\sqrt{2} \theta \psi(y)+\theta^{2} F(y)
$$

$y^{\mu}=x^{\mu}+i \theta \sigma^{\mu} \bar{\theta}$, where $x^{\mu}(\mu=0,1,2,3)$ are the space-time coordinates, and $\theta_{\alpha}, \bar{\theta}_{\dot{\alpha}}(\alpha, \dot{\alpha}=1,2$, spinorial indices $)$ are the fermionic coordinates. $\phi$ is the scalar component of the superfield, $\psi$ is the left-handed chiral fermion component and $F$ is an auxiliary complex scalar field.

Gauge bosons and their fermionic components fit into a so-called vector superfield, which, in the Wess-Zumino gauge [6], can be written in terms of component fields as

$$
V=-\theta \sigma^{\mu} \bar{\theta} A_{\mu}(x)+\left(-i \bar{\theta}^{2} \theta \lambda(x)+H . c .\right)+\frac{1}{2} \bar{\theta}^{2} \theta^{2} D(x)
$$

$A_{\mu}$ is the vector (gauge) field, $\lambda_{\alpha}$ is a left-handed chiral fermion field (gaugino) and $D$ is an auxiliary real scalar field. In the case of nonabelian gauge groups, it is understood that $V \equiv V_{a} T^{a}$, where $T^{a}$ are group generators. Furthermore, the field strength of a given gauge field is part of a chiral superfield $W_{\alpha}$, where $\alpha=1,2$ is a spinorial index, which is given by

$$
W_{\alpha}=-\frac{1}{4} \bar{D}^{2} e^{-2 V} D_{\alpha} e^{2 V}
$$

Where $D, \bar{D}$ are the derivatives with respect to the fermionic directions, and $V$ is a vector superfield. 
In the case of an abelian gauge group, and in the Wess-Zumino gauge,

$$
W_{\alpha}(y)=-i \lambda_{\alpha}(y)+\left(\delta_{\alpha}^{\beta} D(y)-\frac{i}{2}\left(\sigma^{\mu} \bar{\sigma}^{\nu}\right)_{\alpha}^{\beta} F_{\mu \nu}\right) \theta_{\beta}+\theta^{2}\left(\sigma^{\mu} \partial_{\mu} \bar{\lambda}\right)_{\alpha}
$$

Where $F^{\mu \nu}=\partial^{\mu} A^{\nu}-\partial^{\nu} A^{\mu}$ is the field strength tensor for the abelian gauge field $A^{\mu}$.

The most general renormalizable Lagrangian for the MSSM can be written explicitly, with the help of the superfield notation briefly described above and the algebra of the fermionic coordinates, $\theta_{\alpha}$ and $\bar{\theta}_{\dot{\alpha}}[6]$. The only inputs are the gauge group and the matter content.

$$
\mathcal{L}_{\text {MSSM }}=\mathcal{L}_{\text {matter }}+\mathcal{L}_{Y M}+\mathcal{L}_{\text {soft }}
$$

Each one of the components of $\mathcal{L}_{\text {MSSM }}$ will be described in detail below.

The "matter" content of the Lagrangian is contained in $\mathcal{L}_{\text {matter }}$, given by

$$
\begin{aligned}
\mathcal{L}_{\text {matter }}= & \int \mathrm{d}^{4} \theta\left(Q_{i}^{\dagger} e^{-2 V(Q)} Q_{i}+u_{i}^{\dagger} e^{-2 V(u)} u_{i}+d_{i}^{\dagger} e^{-2 V(d)} d_{i}+L_{i}^{\dagger} e^{-2 V(L)} L_{i}\right. \\
& \left.+e_{i}^{\dagger} e^{-2 V(e)} e_{i}+H_{u}^{\dagger} e^{-2 V\left(H_{u}\right)} H_{u}+H_{d}^{\dagger} e^{-2 V\left(H_{d}\right)} H_{d}\right) \\
& +\left(\int \mathrm{d}^{2} \theta W+\text { h.c. }\right)
\end{aligned}
$$

where $i=1,2,3$ is a family index, $Q_{i}$ are the quark doublet chiral superfields, which transform like $(3,2,+1 / 6)$ under the $S U(3)_{c} \times S U(2)_{L} \times U(1)_{Y}$ gauge group, $u_{i}$ are the up-type quark chiral superfields, which transform like $(\overline{\mathbf{3}}, \mathbf{1},-2 / 3), d_{i}$ are the down-type quark chiral superfields, which transform like $(\overline{\mathbf{3}}, \mathbf{1},+1 / 3), L_{i}$ are the lepton doublet chiral superfields, which transform like $(1,2,-1 / 2), e_{i}$ are 
the charged lepton chiral superfields, which transform like $(1,1,+1), H_{u}$ is the uptype Higgs doublet chiral superfield, which transforms like $(1,2,+1 / 2)$, and $H_{d}$ is the down-type Higgs doublet chiral superfield, which transforms like $(1,2,-1 / 2)$. $V(\Phi)$ is short hand notation for $V_{a}^{G} T_{\Phi}^{a}+V_{l}^{W} T_{\Phi}^{l}+V^{B} q_{\Phi} . V$ is the vector superfield containing the $S U(3)_{c}, S U(2)_{L}$, or $U(1)_{Y}$ gauge bosons. $T_{\Phi}$ are the generators of the group in the representation which acts on $\Phi, q_{\Phi}$ is the weak hypercharge of $\Phi$, $a=1 \ldots 8$ colors and $l=1,2,3$.

The superpotential $W$ is given by

$$
\begin{aligned}
W= & \mu H_{u} H_{d}+\lambda_{i j}^{l} L_{i} e_{j} H_{d}+\lambda_{i j}^{d} Q_{i} d_{j} H_{d}+\lambda_{i j}^{u} Q_{i} u_{j} H_{u} \\
& +\tilde{\mu}_{i} H_{u} L_{i}+\tilde{\lambda}_{i j k}^{l} L_{i} L_{j} e_{k}+\tilde{\lambda}_{i j k}^{d} Q_{i} d_{j} L_{k}+\tilde{\lambda}_{i j k}^{q} u_{i} d_{j} d_{k}
\end{aligned}
$$

where the $\lambda$ 's are the Yukawa couplings which yield the fermion masses, $\mu$ is a dimensionful parameter (with dimension of mass). The interactions described in the second line of Eq.(2.1.12) have no SM analog, and are a big source of concern. In particular, a non-zero $\tilde{\lambda}^{l}$ implies violation of lepton number, $\tilde{\lambda}^{q}$ violation of baryon number and $\tilde{\lambda}^{d}$ violation of both.* Constraints, mainly from flavor changing neutral current processes and proton decay, on some of these baryon/lepton number violating couplings are particularly severe [8]. Fig. 2.1 depicts one of the contributions to proton decay due to the $\tilde{\lambda}^{d}$ and $\tilde{\lambda}^{q}$ interactions.

One can get rid of the "tilde" couplings in Eq.(2.1.12) by imposing a global symmetry, namely R-parity, on the Lagrangian. R-parity is a gobal symmetry

*Note that the $\tilde{\mu}_{i} H_{u} L_{i}$ can be made to vanish by appropriately gauge rotating the $L_{i}$ and $H_{u}$ superfields. 


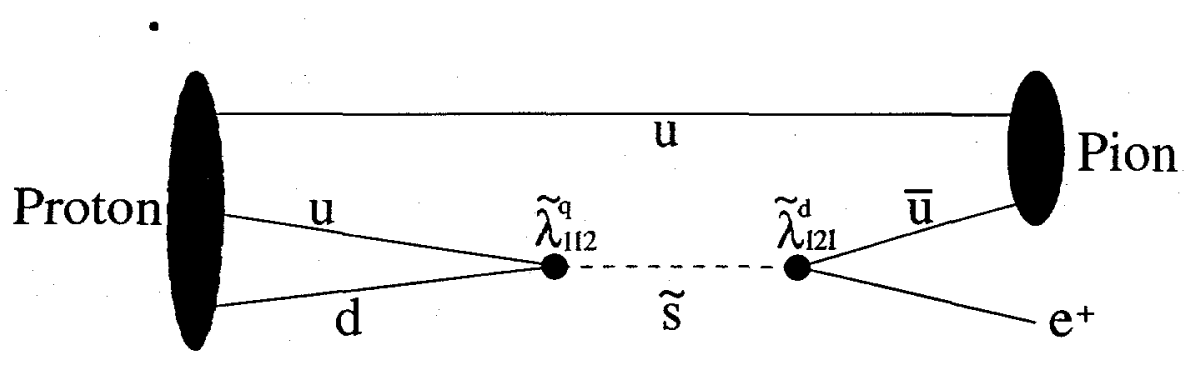

Figure 2.1: One of the many proton decay diagrams which arise in the MSSM in the absence of R-parity.

which does not commute with supersymmetry (SUSY), and therefore particles and their supersymmetric partners have different charges. If one chooses charges such that the "normal" SM fields (quarks, gauge bosons, Higgs scalars) have charge +1 and all other fields have charge -1 , the second line in Eq.(2.1.12) is forbidden. An interesting consequence of R-parity is that superpartners can only be pair produced, and that the lightest superpartner is absolutely stable. This feature plays a fundamental role in cosmology and in collider searches for SUSY.

It is important to remember that, in the SM, all the renormalizable interactions allowed by the gauge symmetries are present. All global symmetries, such as lepton and baryon number, are accidental, and do not have to be imposed a priori. In the MSSM, on the other hand, it seems that imposing R-parity is necessary for rendering it phenomenologically viable.

The Young-Mills part of the Lagrangian, $\mathcal{L}_{Y M}$, contains the kinetic energy and self interaction terms for the gauge bosons and their superpartners.

$$
\mathcal{L}_{Y M}=\int \mathrm{d}^{2} \theta \frac{1}{16 g_{3}^{2}} W_{a}^{\alpha} W_{\alpha}^{a}+\frac{1}{16 g^{2}} W_{i}^{\alpha} W_{\alpha}^{i}+\frac{1}{16 g^{\prime 2}} W^{\alpha} W_{\alpha}+\text { h.c. },
$$

where $W_{\alpha}^{a}, a=1 \ldots 8$ are the $S U(3)_{c}$ chiral superfield field strengths, $W_{\alpha}^{i}, i=$ 
$1 \ldots 3$ are the $S U(2)_{L}$ chiral superfield field strengths and $W_{\alpha}$ is the $U(1)_{Y}$ field strength chiral superfield. $g$ 's are the appropriate gauge coupling constants. Note that the gauge fields and their SUSY partners are not canonically normalized. This can be done by redefining $V \rightarrow g V$.

In order for the MSSM to be phenomenologically viable, it is necessary to break SUSY at some high scale. The main reason for this is that SUSY implies that fermions and their superpartnes have the same mass, and, e.g., a light ( $m=511 \mathrm{keV})$ scalar electron is experimentally ruled out. SUSY breaking can be parameterized at low energies by a set of explicitly SUSY breaking terms. It is important, however, that the explicit SUSY breaking parameters do not reintroduce quadratic divergences in the theory. Terms which fall in this category are referred to as "soft." The soft SUSY breaking part of the Lagrangian, $\mathcal{L}_{\text {soft }}$, is given by

$$
\begin{aligned}
-\mathcal{L}_{\text {soft }}= & \frac{1}{2}\left(M_{\tilde{g}} \tilde{g}^{2}+M_{\tilde{w}} \tilde{w}^{2}+M_{\tilde{b}} \tilde{b}^{2}\right)+m_{H_{d}}^{2}\left|H_{d}\right|^{2}+m_{H_{u}}^{2}\left|H_{u}\right|^{2} \\
& +m_{\tilde{Q}}^{2 i j} \tilde{Q}_{i}^{\dagger} \tilde{Q}_{j}+m_{\tilde{L}}^{2 i j} \tilde{L}_{i}^{\dagger} \tilde{L}_{j}+m_{\tilde{u}}^{2 i j} \tilde{u}_{i}^{\dagger} \tilde{u}_{j}+m_{\tilde{d}}^{2 i j} \tilde{d}_{i}^{\dagger} \tilde{d}_{j}+m_{\tilde{e}}^{2 i j} \tilde{e}_{i}^{\dagger} \tilde{e}_{j} \\
& -m_{3}^{2} H_{u} H_{d}+\mathcal{A}_{d}^{i j} \tilde{Q}_{i} \tilde{d}_{j} H_{d}+\mathcal{A}_{u}^{i j} \tilde{Q}_{i} \tilde{u}_{j} H_{u}+\mathcal{A}_{l}^{i j} \tilde{L}_{i} \tilde{e}_{j} H_{d},
\end{aligned}
$$

where $\tilde{g}$ are the gluino fields, $\tilde{w}$ are the $\mathrm{W}$-ino fields, and $\tilde{b}$ is the B-ino field. In order to solve the gauge hierarchy problem, SUSY breaking parameters must be of $O(1) \mathrm{TeV}$ or less.

It is important to check if electroweak symmetry breaking can occur within the MSSM. This is, of course, required in order to make the model phenomenologically viable. 
The tree-level Higgs potential of the MSSM is given by

$$
\begin{aligned}
V= & m_{1}^{2}\left|H_{d}\right|^{2}+m_{2}^{2}\left|H_{u}\right|^{2}-m_{3}^{2}\left(H_{d} H_{u}+\text { c.c. }\right)+ \\
& +\frac{g_{2}^{2}}{8}\left(H_{d}^{\dagger} \vec{\sigma} H_{d}+H_{u}^{\dagger} \vec{\sigma} H_{u}\right)^{2}+\frac{g^{\prime 2}}{8}\left(\left|H_{d}\right|^{2}-\left|H_{u}\right|^{2}\right)^{2},
\end{aligned}
$$

where

$$
\begin{aligned}
& m_{1}^{2}=\mu^{2}+m_{H_{d}}^{2}, \\
& m_{2}^{2}=\mu^{2}+m_{H_{u}}^{2} .
\end{aligned}
$$

In the MSSM, one can show that the vacuum can always be gauge rotated to the following configuration

$$
H_{d}=\left(\begin{array}{c}
v_{d} \\
0
\end{array}\right), \quad H_{u}=\left(\begin{array}{c}
0 \\
v_{u}
\end{array}\right) .
$$

It is assumed that no other scalar fields, such as squarks and sleptons, acquire non-zero vacuum expectation values. This has to be checked in order to guarantee that the color gauge group is unbroken and baryon/lepton number is conserved. The two expectation values need to satisfy $v_{d}^{2}+v_{u}^{2}=v^{2}=(174 \mathrm{GeV})^{2}$ in order to reproduce the observed $M_{Z}$, and it is conventional to parameterize them by $v_{d}=v \cos \beta, v_{u}=v \sin \beta$. The minimization condition of the potential can be rewritten in the following form:

$$
\begin{aligned}
\frac{M_{Z}^{2}}{2} & =-\mu^{2}+\frac{m_{H_{d}}^{2}-m_{H_{u}}^{2} \tan ^{2} \beta}{\tan ^{2} \beta-1}, \\
2 m_{3}^{2} & =\left(2 \mu^{2}+m_{H_{d}}^{2}+m_{H_{u}}^{2}\right) \sin 2 \beta .
\end{aligned}
$$

It is important to note that $\mu^{2}$ is positive definite because $\mu$ is a parameter in the superpotential. It turns out that $m_{3}^{2} /(\sin 2 \beta)$ must also be positive in order to 
avoid a runaway behavior in the Higgs potential. Electroweak symmetry breaking occurs, therefore, if Eqs. (2.1.19) and (2.1.20) can be simultaneously satisfied.

Eqs. (2.1.19) and (2.1.20) are more readily satisfied if $-m_{H_{u}}^{2}$ is positive at the electroweak symmetry breaking scale. Interestingly enough, it seems that the MSSM has a tendency to favor $-m_{H_{u}}^{2}>0$ at the weak scale, even if $+m_{H_{u}}^{2}>0$ at a higher energy scale. Note also that, in the absence of SUSY breaking terms, electroweak symmetry is not be broken. It is, therefore, part of the common lore that the MSSM plus SUSY breaking leads "naturally" to electroweak symmetry breaking, and that SUSY breaking "triggers" electroweak symmetry breaking. This issue will be further analyzed and this lore criticized in future chapters.

After electroweak symmetry breaking, one can identify the remaining propagating degrees of freedom and their masses [7]. They are:

(1) the usual SM fermions, the quarks and the leptons, which acquire masses through interactions with the Higgs boson fields, contained in the superpotential (Eq. (2.1.12)). Note that in order for all fermions to become massive, both Higgs doublets must acquire non-zero vacuum expectation values. This is one of the reasons why there are two Higgs doublets in the MSSM. Another reason is to cancel the gauge anomalies introduced by the fermionic partner of one Higgs boson.

(2) squarks and sleptons, the scalar partners of "ordinary" matter. These are usually denoted by the corresponding SM fermion symbol with a tilde (e.g. $\tilde{\mu}_{L}$ is the supersymmetric partner of the left-handed muon, the "smuon"). Note that they already have SUSY breaking mass-squared parameters, and they acquire 
"left-right" ${ }^{\dagger}$ masses after electroweak symmetry breaking.

(3) the gluino (usually referred to as $\tilde{g}$ ), the supersymmetric partner of the gluon, which has a SUSY breaking mass $M_{\tilde{g}}$ (see Eq.(2.1.14)). Note that the gluino is a Majorana $S U(3)_{c}$ octet fermion.

(4) the supersymmetric partners of the electroweak gauge bosons (usually referred to as W-inos, $\tilde{w}_{1,2,3}$ and B-ino $\tilde{b}$ ) and the Higgs bosons (Higgsinos, $\tilde{H}_{u, d}$ ), which acquire mixing mass terms after electroweak symmetry breaking. The mass matrices can be diagonalized and the final propagating degrees of freedom are two "charginos," $\tilde{\chi}_{1,2}^{ \pm}$, which are Dirac fermions, and four Majorana neutral fermions, the "neutralinos," $\tilde{\chi}_{1,2,3,4}^{0}$. The interactions of the charginos and neutralinos depend heavily on the mixing parameters.

(5) the Higgs scalars, which are the analog of the Higgs boson in the SM. Because there are two Higgs scalar fields which acquire vacuum expectation values, there are five real degrees of freedom left after electroweak symmetry breaking (three are "eaten" by the weak vector bosons). They are: two real scalars, the "light" Higgs scalar, $h^{0}$ and the "heavy" Higgs scalar, $H^{0}$, one pseudo-scalar, $A^{0}$ and one charged scalar, $H^{ \pm}$. It is easy to compute, at tree level, the mass of the light Higgs boson

$$
m_{h^{0}}^{2}=\frac{1}{2}\left(M_{A}^{2}+M_{Z}^{2}-\sqrt{\left(M_{A}^{2}+M_{Z}^{2}\right)^{2}-4 M_{Z}^{2} M_{A}^{2} \cos ^{2} 2 \beta}\right),
$$

tIt is clear that scalar fields do not have a "handedness" degree of freedom. It is, however, common to refer to right/left-handed sfermions in the sense that they are partners of particular right/left-handed chiral fermions. 
where $M_{Z}^{2}$ is the $Z^{0}$ vector boson mass-squared and $M_{A}^{2}=2 m_{3}^{2} /(\sin 2 \beta)$ (see Eq. (2.1.20)) is the pseudo-scalar Higgs mass-squared. One can therefore verify that, at tree level, $m_{h^{0}}^{2} \leq M_{Z}^{2}$. Since the light Higgs boson is in general very similar to the SM Higgs boson, the MSSM would be almost completely ruled out by SM Higgs boson searches [3]. Quantum corrections, however, have a tendency to push the light Higgs boson mass up, and the constraint above is somewhat alleviated. Still, the light Higgs boson mass is relatively light, and, in the MSSM, cannot exceed $m_{h^{0}} \lesssim 130 \mathrm{GeV}[9]$. Note that this constraint seems to agree with the present day electroweak precision data analysis [10], which currently indicates the existence of a light Higgs boson.

\subsection{What is known about the MSSM}

In this section, a very brief description of what is known about the MSSM, in particular its parameters such as scalar masses and mixing angles, is given. A few constraints from rare/forbidden processes will be mentioned, along with a few of the most popular search strategies at accelerator facilities.

The MSSM, briefly introduced in the previous section, has a huge number of parameters. In particular, one can easily note that the soft SUSY breaking part of the Lagrangian (Eq.( 2.1.14)) alone contains over 100 complex parameters! Most of these are severely constraint by rare or forbidden processes [11].

Some of the flavor non-diagonal components of the soft scalar mass-squared parameters lead to unacceptable flavor changing neutral currents. A non-zero $\left(m_{\bar{e}}^{2}\right)^{12}$ 


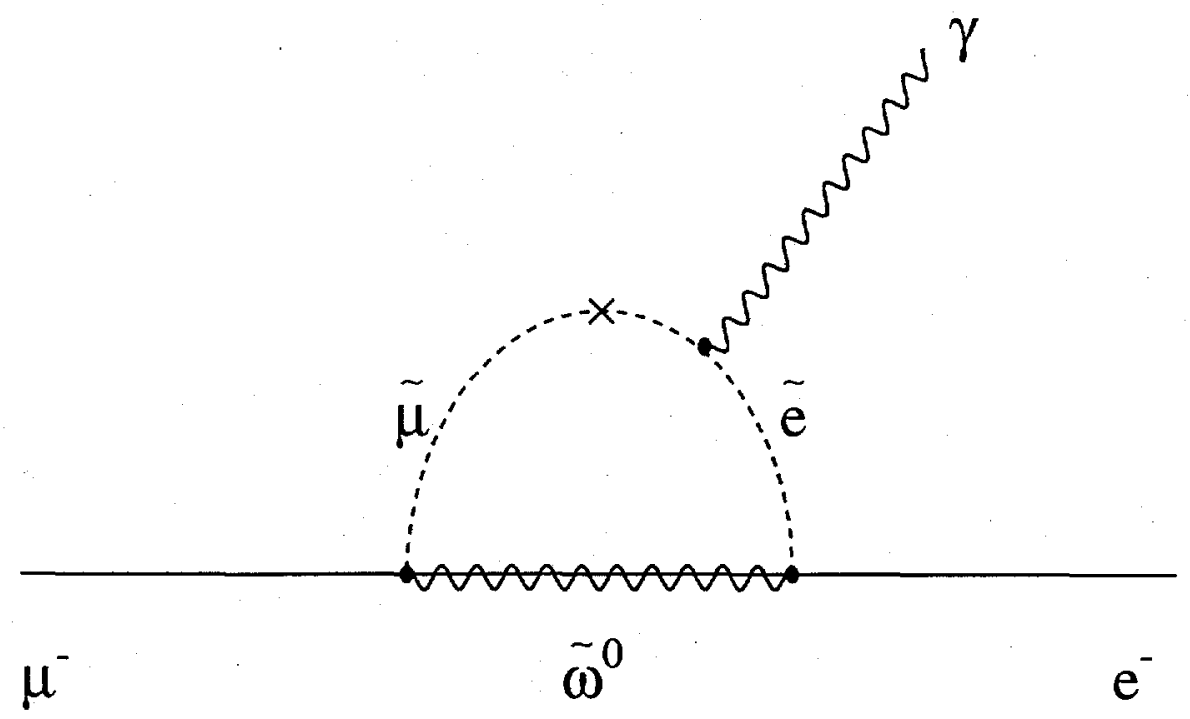

Figure 2.2: One of the SUSY contributions to $\mu^{-} \rightarrow e^{-} \gamma$. The cross represents the insertion of the $\left(m_{\tilde{e}}^{2}\right)^{12}$ off-diagonal slepton mass-squared parameter (see Eq. (2.1.14)).

can lead to a large $\mu \rightarrow e \gamma$ (muon and electron number violation) branching ratio. Fig. 2.2 illustrates one of the dominant contributions, while other contributions come from virtual sneutrinos and neutralinos running in the loop. The cross indicates the insertion of the flavor changing parameter $\left(m_{\tilde{e}}^{2}\right)^{12} \tilde{e} \tilde{\mu} . \mu \rightarrow e \gamma$ is a particularly powerful way of seaching for flavor changing interactions, given that theoretical predictions are particularly clean and are not plagued by strong interaction long-distance effects. See $[12,13]$ for details and numerical constraints on SUSY breaking parameters.

Another big constraint to the MSSM parameter space comes from $K^{0} \leftrightarrow \bar{K}^{0}$ mixing and CP violation. Fig. 2.3 depicts one of the many SUSY contributions 


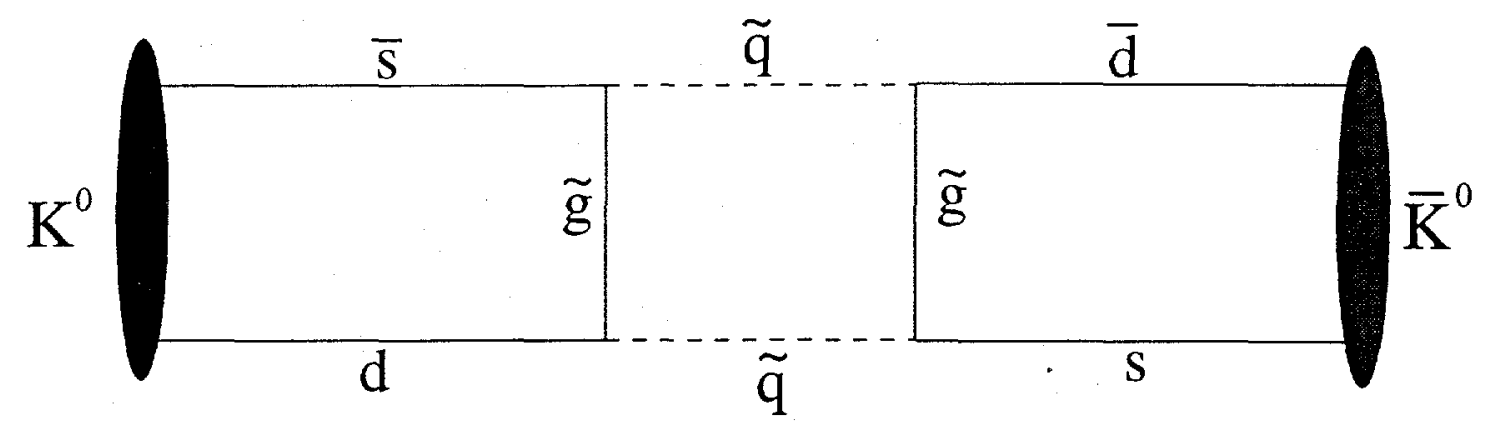

Figure 2.3: One of the SUSY contributions to $K^{0} \leftrightarrow \bar{K}^{0}$ mixing. $\tilde{q}$ represents any of the squarks. Here, the flavor-changing effects have been rotated to the $\tilde{g} \tilde{q} q^{\prime}$ couplings.

to $K^{0} \leftrightarrow \bar{K}^{0}$ mixing. Note that, unlike in Fig. 2.2, in Fig. 2.3 the flavor changing interactions have been "rotated" to the quark-squark-gluino vertex. This happens because the unitary rotation that diagonalizes the quark mass matrix is not necessarily equal to the one that diagonalizes the squark mass matrix, due to the SUSY breaking parameters in Eq.(2.1.14). This is similar to what happens in weak interactions, where the unitary rotation that diagonalizes the up-type masses is different from the one that diagonalizes down-type masses. The end result is flavor changing neutral currents mediated by weak effects [1]. For a quantitative description of constraints on the MSSM parameter space due to flavor changing neutral currents and Cp-violation in the kaon system, see for example [13].

Direct searches for supersymmetric particles are also underway, mostly at the LEP (Large Electron-Positron) collider at CERN and the Tevatron collider at Fermilab. Future experiments, such as the LHC (Large Hadron Collider) at CERN and a possible NLC (Next Linear Collider), whose future location is still unknown, 
will spent a large fraction of their resources looking for low-energy SUSY. No sign of superparners has been discovered to the present date, and what the experiments have been able to do is set upper bounds on parameters, such as couplings and masses, of the MSSM. This, in general, tends to be very much model dependent, in particular because the MSSM has too many parameters. Setting model independent bounds on sparticle masses, for example, is very tricky.

A few of the SUSY search strategies for hadron and lepton machines will be briefly presented below. For thorough reviews, see, for example, $[14,15,16]$. For a complete listing of all bounds on superpartner masses and couplings, see [3].

In most of the SUSY collider searches, R-parity is assumed. There are two very important consequences, which have already been briefly mentioned: (i) superpartners can only be produced in pairs at collider facilities and (ii) a superpartner has to decay to another superpartner plus any number of ordinary matter particles. In particular, this implies that the lightest superpartner (LSP) must be stable. Even if a small amount of R-parity violation is allowed, it is usually true that the LSP is long-lived enough that it does not decay inside high energy physics detectors. The effects of a stable LSP on collider experiments depend on whether or not the LSP is charged. A neutral LSP will leave the detector without interacting, and yields a large missing energy signature. An electrically charged LSP will can be detected via $d E / d x$ measurements as a heavy stable charged object. It is interesting to note that cosmological considerations prefer a neutral LSP, in particular the lightest neutralino, $\tilde{\chi}_{1}^{0}$. 
At lepton colliders $[14,16]$, the hope is to produce a pair of superpartners, such as $\tilde{e}^{+} \tilde{e}^{-}, \tilde{\chi}_{1}{ }^{+} \tilde{\chi}_{1}^{-}, \tilde{\nu}_{e}^{+} \tilde{\nu_{e}}{ }^{-}$, etc, and directly look for their decay products. Note that the decay products of each particular superpartner depend on the values of the MSSM parameters. Standard signs for SUSY at lepton colliders include many energetic leptons and large missing energy. As an example, assume the following production and decay processes:

$$
\begin{aligned}
e^{+}+e^{-} \longrightarrow & \tilde{\mu}_{R}+\tilde{\mu}_{R}^{*}, \\
& \tilde{\mu}_{R} \rightarrow \tilde{\chi}_{1}^{0}+\mu^{+},
\end{aligned}
$$

where $\tilde{\chi}_{1}^{0}$ is the LSP. The signal to look for is two energetic muons, plus a large amount of missing energy. The largest physics background one would have to worry about is $W^{+} W^{-}$production, followed by the decay $W^{ \pm} \rightarrow \mu^{ \pm}+\nu_{\mu}\left(\bar{\nu}_{\mu}\right)$. Another model independent constraint comes from the precise measurement of the $Z^{0}$ boson decay width at LEP [3]. This is a particularly robust bound because it consists of an inclusive process, i.e., the result does not depend on the identification and reconstruction of particular final states. For example, all $\tilde{\nu}$ masses are bound to be larger than $\gtrsim 43 \mathrm{GeV}$ because of this measurement. This is similar to the bound on the number of light neutrino species [17].

At hadron colliders $[14,15]$, it is expected that mostly strongly interacting particles, squarks and gluinos, will be produced. Furthermore, it is often thought that squarks and gluinos are heavier than other superpartners, which only interact via electroweak interactions. The reason for this prejudice will be discussed in the 
next chapter. It is therefore assumed that gluinos and squarks will generate long decay chains. Typical SUSY signatures are multi-jet events with large amounts of missing energy or multi-leptons plus jets plus missing energy. The main physics backgrounds come from the production of massive vector bosons which further decay into neutrinos, which carry away the missing energy. As an example, assume

$$
\begin{aligned}
& p+\bar{p} \longrightarrow \tilde{g}+\tilde{g}, \\
& \tilde{g} \rightarrow \tilde{q}+q, \\
& \tilde{q} \rightarrow \tilde{\chi}_{1}^{+}+q^{\prime} \text { and } \tilde{q} \rightarrow \tilde{\chi}_{1}^{0}+q \\
& \tilde{\chi}_{1}^{+} \rightarrow \tilde{\chi}_{1}^{0}+q+q^{\prime},
\end{aligned}
$$

where $\tilde{\chi}_{1}^{0}$ is the LSP. The final state for the decay chain outline above is 6 jets plus missing energy.

Another sigature of SUSY at hadron machines is 3 charged leptons plus missing energy [18]. This comes from the production of a virtual $W$-boson, which decays into $\chi_{1}^{+}+\chi_{2}^{0}$. This is likely to happen if $\chi_{1}^{+}\left(\chi_{2}^{0}\right)$ has a large W-ino (Z-ino) component. It is further assumed that $\chi_{1}^{+} \rightarrow l+\nu_{l}+\chi_{1}^{0}$ and $\chi_{2}^{0} \rightarrow l+\bar{l}+\chi_{1}^{0}$. In some of the most popular parameterizations of the MSSM (see next chapter) this a realistic possibility. Its biggest advantage is the fact that it is very clean, and almost background free. The only physics background consists of $W^{ \pm}+Z^{0}$ production, with both $W$ and $Z$ decaying leptonically. It is, however, easy to remove this background by imposing that the invariant mass of each lepton pair is different from the $Z$-boson mass. 
In summary, there are already several constraints on the MSSM parameter space, some of them very strong. On the other hand, the MSSM has too many parameters, and it is hard to constrain those parameters in a model independent way. Finally, it is worthwhile to mention that, if some sign of new physics is discovered in the next generation of collider, it is still very challenging to determine if SUSY has been discovered or not. Furthermore, even if it is established that low-energy SUSY has been detected, determining the MSSM parameters is still very challenging (see [19] for a study of how SUSY parameters might be measured at the LHC).

\subsection{Excluding Light Gluinos from Z Decays}

Supersymmetry is one of the primary targets of extensive searches at various collider experiments, most importantly at the CERN $e^{+} e^{-}$collider, LEP, and the Fermilab $p \bar{p}$ collider, Tevatron $[14,15]$. Negative searches at these and previous colliders have already put significant constraints on the parameter space of lowenergy supersymmetry, as briefly described earlier. However, a light gluino below the few $\mathrm{GeV}$ mass range has surprisingly weak experimental constraints as emphasized recently by various authors $[20,21,22]$ (see, however, an opposing view [23]). It is an extremely important task to verify or exclude a gluino in this light window experimentally. While the Tevatron Run II is expected to extend the reach of heavy gluinos up to a few hundred $\mathrm{GeV}$, little effort is devoted to defini-

tively exclude or verify the light gluino window. On the other hand, a careful 
reexamination of the existent data may reveal an overlooked constraint on a light gluino; this is the motivation to study the existent data in detail.

In this section the published data on $Z$ decays into four jets $[24,25,26,27$, $28,29]$ is reanalyzed, and it is found that they already exclude a gluino lighter than $1.5 \mathrm{GeV} / c^{2}$ at more than $90 \%$ confidence level. It is assumed that the gluino does not decay inside the detector. Since the published results use only 1991 and 1992 data, it is conceivable that the currently available data, if analyzed properly, could put a much more significant constraint on a light gluino.

First, the existent constraints on a light gluino are briefly reviewed (see $[21,30]$ for more details). The negative searches at beam dump experiments have excluded a light gluino which decays inside the detector into photino, which in turn interacts with the neutrino detector. However, a gluino tends to leave the detector without decaying if the squark mass is above a few hundred $\mathrm{GeV} / c^{2}[31,32]$. Even if the gluino decays, the photino interacts very weakly in this case and cannot be detected. If the gluino does not decay, it forms bound states such as gluinoball $\tilde{g} \tilde{g}$, glueballino $g \tilde{g}$ or baryon-like states, especially $u d s \tilde{g}[33]$. Other states are likely to decay into these neutral bound states, and searches for exotic charged hadrons may not apply unless a charged gluino bound state decays only weakly. On the other hand, the mass region above $1.5 \mathrm{GeV} / c^{2}$ and below $4 \mathrm{GeV} / c^{2}$ is excluded from quarkonium decay $\Upsilon \rightarrow \gamma \eta_{\tilde{g}}$, where $\eta_{\tilde{g}}$ is the pseudo-scalar gluinoball, independent of the gluino lifetime $[34,21]$. Whether the bound extends to lower masses is controversial because of the applicability of perturbative QCD calculations [34]. 
The mass range above $4 \mathrm{GeV} / c^{2}$ is expected to give a shorter lifetime and is excluded by a negative search for events with missing energy at UA1 [35]. The authors of [36] claim that the limit from UA1 extends down to $3 \mathrm{GeV} / c^{2}$. In any case, the least constrained region is the mass range below $1.5 \mathrm{GeV} / c^{2}$, where the gluino is relatively stable so that it does not decay inside detectors. This is the window of interest in this section.

It is important to emphasize that the best method to exclude the gluino mass range below $1.5 \mathrm{GeV} / c^{2}$ is to use inclusive processes rather than searching for specific bound states with certain decay modes. The latter search would heavily depend on assumptions such as the mass spectrum of various gluino bound states and their decay modes and decay lifetimes. One would have to design experiments and put constraints with all possible theoretical assumptions on gluino bound states in order to exclude the light gluino definitively. On the other hand, the constraints would be much less sensitive to theoretical assumptions if they were based on inclusive processes where perturbative QCD is applicable. There are several possibilities pointed out in the literature along this line. The most popular one is to study the effect of light gluinos in the running of the QCD coupling constant $\alpha_{s}$. It was even pointed out that the values of $\alpha_{s}$ from higher energy measurements tend to be higher than those extrapolated from lower energies using QCD with the ordinary quark flavors, and that the data actually prefer the existence of a light gluino to compensate the slight discrepancy $[20,37,38]$. However, this issue remains controversial $[39,40,41]$. Even though the discrepancy 
between low-energy and high-energy measurements is diminishing [42], still the data are not precise enough to exclude or verify a light gluino definitively. The second one is its effect on the Altarelli-Parisi evolution of the nucleon structure functions $[43,44]$. Unfortunately the effect is too small to be tested using the present experimental data. It might be that the more recent HERA data could improve the situation, but making a definite statement on the existence of a light gluino appears to be difficult. The third one is to study the angular correlations in the so-called " $3+1$ " jet events at HERA [45]. However, the effect of the light gluino was found to be negligible. The final one, which is employed in this section, is the study of four jet correlations in $e^{+} e^{-}$collisions $[46,41,47]$. Previous studies did not find significant constraints, but given the size of the current LEP data, this seems to be the most promising direction.

The only data used in this section are studies of QCD color factors $[27,28$, 29]. The experimental groups at LEP have performed impressive analyses of the hadronic $Z$ decays into four jets, extracting QCD color factors $C_{A} / C_{F}$ and $T_{F} / C_{F}^{\ddagger}$ from jet angular distributions, to confirm $S U(3)$ as the QCD gauge group and five light quark flavors. The angular distributions of $q \bar{q} q \bar{q}$ final state differ from those of $q \bar{q} g g$, where $q$ refers to a generic quark and $g$ to a gluon. Three angles are commonly used in four-jet analyses: the Bengtsson-Zerwas (BZ) angle $\chi_{B Z}$ [48],

\footnotetext{
\$The QCD color factors are defined by $C_{F} \mathbf{1}=\sum_{a} T^{a} T^{a}$ and $T_{F} \delta^{a b}=\operatorname{Tr}\left(T^{a} T^{b}\right)$ for the fundamental representation, and $C_{A} \delta^{a b}=\operatorname{Tr}\left(T^{a} T^{b}\right)$ for the adjoint representation. $T^{a}(a=$ $1, \ldots, 8)$ are group generators in each representations.
} 
the modified Nachtmann-Reiter (NR) angle $\theta_{N R}^{*}[49]$, and the opening angle of the two less energetic jets $\alpha_{34}$. If there exists a light gluino $\tilde{g}$, the final state $q \bar{q} \tilde{g} \tilde{g}$ also contributes to the $Z$ decays into four jets. The angular distributions of $q \bar{q} \tilde{g} \tilde{g}$ would be identical to those of $q \bar{q} q \bar{q}$. Therefore, a possible light gluino would change the extracted $T_{F} / C_{F}$ but not $C_{A} / C_{F}$. Apart from the mass effects, $T_{F} / C_{F}$ should increase by a factor of $(5+3) / 5$, because the gluino is a color-octet and counts effectively as three additional massless quarks. Note that these analyses do not use the overall rate of four-jet events since it is sensitive to the choice of $\alpha_{s}$ in the absence of next-to-leading order (NLO) calculations. So far the experimental analysis which used the highest statistics is the one by OPAL [29], which also briefly discussed constraints on a light gluino. They found that the light gluino is barely outside the $68 \%$ confidence level contour and decided the data did not put a significant constraint.

However, most of the previous analyses are not carefully designed to study the effect of a light gluino because of the following reason. When one discusses a possible light gluino, QCD with the color group $S U(3)$ should be assumed. Given overwhelming experimental evidences of QCD, it is not wise to, for instance, vary the number of colors $N_{c}=3$ when one studies the effect of a particle (light gluino) added to $\mathrm{QCD}$. Therefore, the QCD color factor $C_{A} / C_{F}$ is fixed to be that of the $S U(3)$ group, $9 / 4$. Second, it is already known that there are five quark flavors $u$, $d, s, c$ and $b$, which appear in $Z$ hadronic decays. When one puts constraints on an additional contribution from a light gluino, one should not vary the number 
of flavors below 5 , or equivalently, $T_{F} / C_{F}$ below $3 / 8$. The only LEP paper which analyzed data in a way close to this spirit, and put an upper bound on possible additional $q \bar{q} q \bar{q}$-type final states, is the one from OPAL [25]; but it used very limited statistics. All more recent papers $[27,28,29]$ varied both $C_{A} / C_{F}$ and $T_{F} / C_{F}$ without constraints. By reanalyzing data with these constraints a much more significant bound on a light gluino than reported can be placed. Actually, fixing the group to be $S U(3)\left(C_{A} / C_{F}=9 / 4\right)$ has the greatest impact on the confidence level, while restricting $T_{F} / C_{F} \geq 3 / 8$ has a much smaller effect (actually it makes the significance worse). Furthermore the finite mass of the bottom quark is included in the analysis, which slightly improves the significance. Overall, a massless gluino is excluded already better than at $90 \%$ confidence level by the OPAL 1991 and 1992 data only [29].

The reported contour on the $C_{A} / C_{F}, T_{F} / C_{F}$ plane is shown in Fig. 2.4. $C_{A} / C_{F}=9 / 4$ is fixed because of the philosophy of the present study, stated above. Since one-dimensional $\chi^{2}$ distributions have much higher confidence levels than two-dimensional ones, this change improves the significance of the data drastically. From their $\chi^{2}$ contours, $\chi^{2}$ is minimized with fixed $C_{A} / C_{F}=9 / 4$, and $\Delta \chi^{2}$ is defined relative to the $\chi^{2}$ at the minimum $\left(T_{F} / C_{F}=0.36\right)$. The confidence levels are calculated using a one-dimensional $\chi^{2}$ distribution with $\Delta \chi^{2}$ defined in this manner. This is a conservative choice because $\Delta \chi^{2}<\chi^{2}$. One obtains $T_{F} / C_{F}=0.36 \pm 0.15$ with fixed $C_{A} / C_{F}$. If one had used this central value and the standard deviation, a massless gluino would be excluded at $95 \%$ confidence 
level. However, one also needs to impose another constraint, $T_{F} / C_{F} \geq 3 / 8$, which can be easily taken into account. The standard method is to use the Gaussian distribution only in the physical region, and scale the normalization of the distribution so that the total probability in the physical region becomes unity. Since the central value is very close to the theoretical value of the $\mathrm{QCD}$, this effectively increases the probability of allowing light gluinos by a factor of two; numerically the confidence level is $88 \%$.

Finally, the effect of the finite mass of the bottom quark and gluinos on the extracted $T_{F} / C_{F}$ are studied. The authors of [50] studied the effect of the finite mass of quarks on the four-jet rates. They also looked at the angular distributions and reported there were little changes. Even though it is true that the distributions do not change drastically, they gradually become similar to those of $q \bar{q} g g$ final state as one increases the mass of the quark, and hence the extracted $T_{F} / C_{F}$ from the fit to the distributions has a relatively large effect due to the finite mass of the bottom quark. The papers $[27,28]$ do not take this effect into account at all. The OPAL experiment [29] used parton level event generators by the authors of [50] and [47] to study the effect. They have found a surprisingly large effect: the bottom quark contribution to $T_{F} / C_{F}$ was about one half of a massless quark at $y_{c u t}=0.03$. Their estimate was confirmed in a detailed parton-level calculation based on the done in [51], neglecting the interference between primary and secondary quarks. This approximation is known to be better than a few percent. On the other hand, this approximation has the clear advantage of enabling us to distinguish primary 


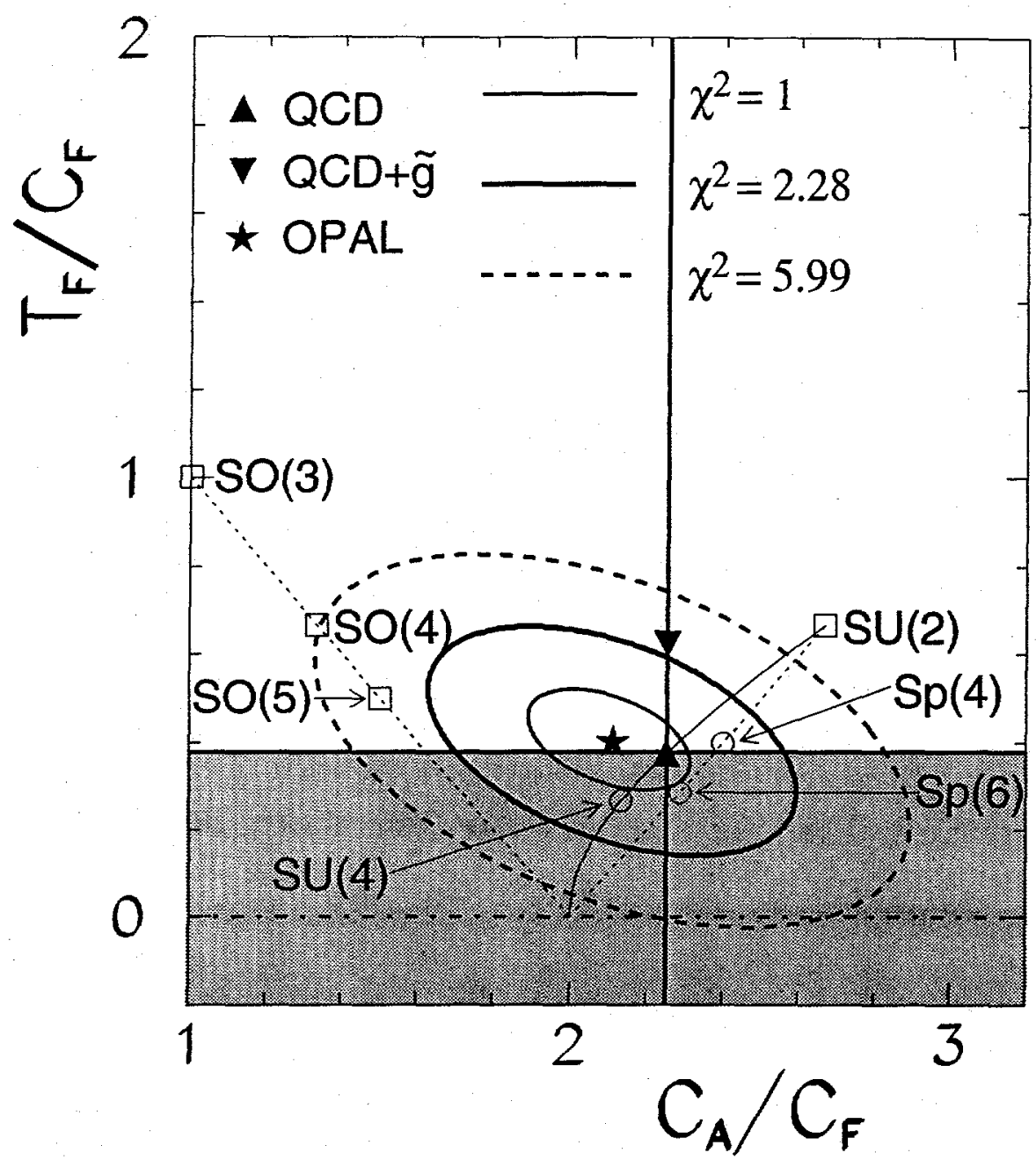

Figure 2.4: Extracted QCD color factors from the OPAL analysis [29]. The shown $\chi^{2}$ values correspond to $39.3 \%, 68 \%$ and $95 \%$ confidence levels with two degrees of freedom. The constraint $C_{A} / C_{F}=9 / 4$ is imposed (vertical solid line) and one is limited to the unshaded region $\left(T_{F} / C_{F} \geq 3 / 8\right)$ in order to put constraints on a possible light gluino contribution to the four-jet events from $Z$ decays. See the text for more details. 
and secondary quarks unambiguously. The numerical code used in this section employs helicity amplitude techniques using the HELAS package [52], which made it straight-forward to incorporate finite masses in the four-jet distributions.

The finite mass affects the extracted $T_{F} / C_{F}$ in two ways. First, the rate of producing secondary massive quarks is suppressed compared to the massless case as shown with the solid line in Fig. 2.5. For instance, there is about $20 \%$ suppression with $m_{q}=5 \mathrm{GeV} / c^{2}$ and $y_{c u t}=0.03$. This result is consistent with [50]. The mass of the primary quark has little effect on the rate: only a $6 \%$ suppression for $m_{q}=5 \mathrm{GeV} / c^{2}$. That the distributions in. $\mathrm{BZ}$ and $\mathrm{NR}$ angles with a massive primary quark are indistinguishable from the massless case was also checked. These observations are consistent with naive expectations, because the primary quarks are much more energetic than the secondary ones and hence the mass effect is suppressed by $m^{2} / E^{2}$. Therefore the finite mass of primary quarks is neglected hereafter: Second, the NR and BZ angle distributions gradually approach those of the $q \bar{q} g g$ final state as one increases the mass of the secondary quarks. We are not aware of detailed analyses of these distributions with massive quarks in the literature. The distributions are shown in Fig. 2.6 normalized so that the total area below the curve is unity, in order for the effect on the rate and that on the distribution to be clearly separated. The distributions are fit as linear combinations of $q \bar{q} g g$ and massless $q \bar{q} q \bar{q}$ distributions to determine the effective $T_{F} / C_{F}$, in order to mimic the experimental analyses. The fit is surprisingly good; this was checked for quark masses between 0 and $5 \mathrm{GeV} / c^{2}$. Combined with the 
reduction in the rate, the net effect of the finite mass of secondary quarks is shown in Fig. 2.5. With $m_{b}=5 \mathrm{GeV} / c^{2}$ for secondary bottom quarks, the overall rate of $q \bar{q} b \bar{b}$ final state is reduced to $82.5 \%$, while the fit to angular distributions gives a $T_{F} / C_{F}$ reduced to $76.4 \%(\mathrm{BZ})$ or $85.5 \%$ (NR) compared to that of a massless quark flavor (3/8), on top of the reduction in the rate. In total, secondary bottom quarks contribute to $T_{F} / C_{F}$ as $3 / 8 * 0.630$ or $3 / 8 * 0.705$, which is not a negligible suppression. The extracted $T_{F} / C_{F}$ from the data is an average of $T_{F} / C_{F}$ from five flavors. The reported $T_{F} / C_{F}$ in [29] includes a correction to compensate the apparent suppression due to the finite bottom quark mass. Such a correction in turn effectively enhances the additional contribution from gluinos by a factor of $5 /(4+0.630)$ or $5 /(4+0.705)$. Note that this slight enhancement effect does not change significantly even when one varies $m_{b}$ from 4 to $5 \mathrm{GeV} / c^{2}$, as can be seen in Fig. 2.5.

The actual OPAL analysis [29] fits the data in the three dimensional space spanned by BZ, NR and $\alpha_{34}$ angles after bin-by-bin systematic corrections from Monte Carlo simulations. Such an analysis is beyond the scope of this dissertation. The total effect of the finite mass is assumed to be somewhere between the effects on $\mathrm{BZ}$ or NR angles since $\alpha_{34}$ is not as effective in extracting $T_{F} / C_{F}$. As it is clear from Fig. 2.6, fits to distributions of massive quarks give apparent additional contributions to $q \bar{q} g g$ and hence $C_{A} / C_{F}$. They are completely negligible, however, compared to the size of the true $q \bar{q} g g$ which is about one order of magnitude larger than the sum of all $q \bar{q} q \bar{q}$ final states, and hence such contributions will be 


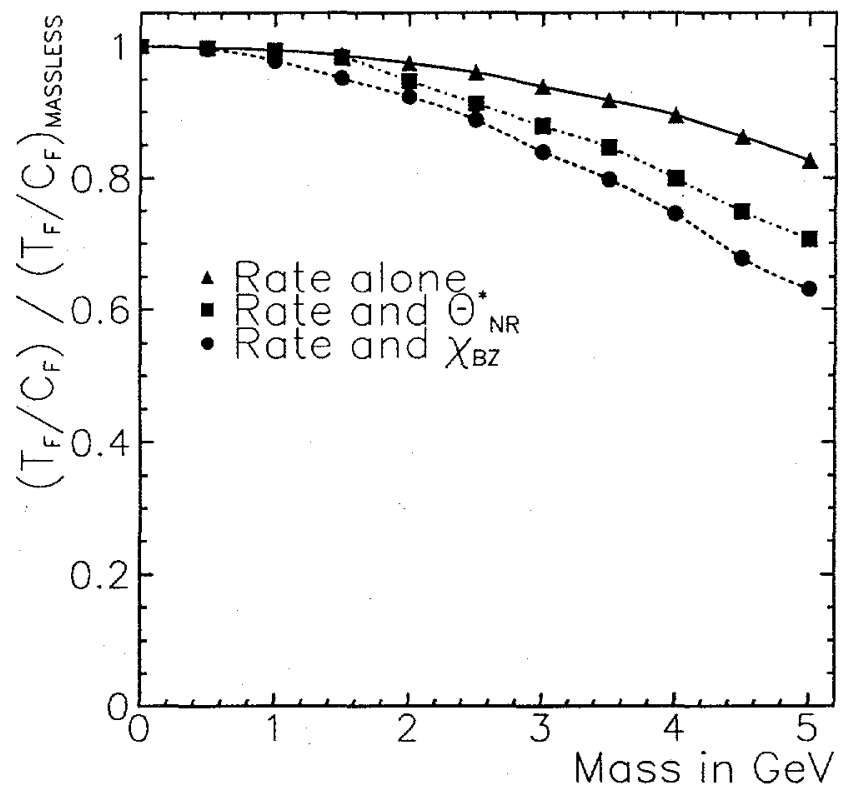

Figure 2.5: Effective contribution to $T_{F} / C_{F}$ of a massive secondary quark relative to the massless case. The solid line shows the reduction in the rate alone. The other two lines include the effect that the distributions in BZ and NR angles change due to finite quark mass. $y_{\text {cut }}=0.03$ and $\sqrt{s}=m_{Z} c^{2}=91.17 \mathrm{GeV}$ was assumed.

neglected hereafter.

Given the above considerations, the exclusion confidence levels on a light gluino for varying gluino masses is presented in Fig. 2.7. For both curves, $m_{b}=5 \mathrm{GeV} / c^{2}$ was used together with the effective $T_{F} / C_{F}$ extracted from the fits to $\mathrm{BZ}$ and NR angles. The finite mass effect of the gluino is treated in the same manner. First of all, it is clear that the finite mass effect which was studied depends little on the choice of BZ or NR angles, and hence we believe it mimics the true experimental 

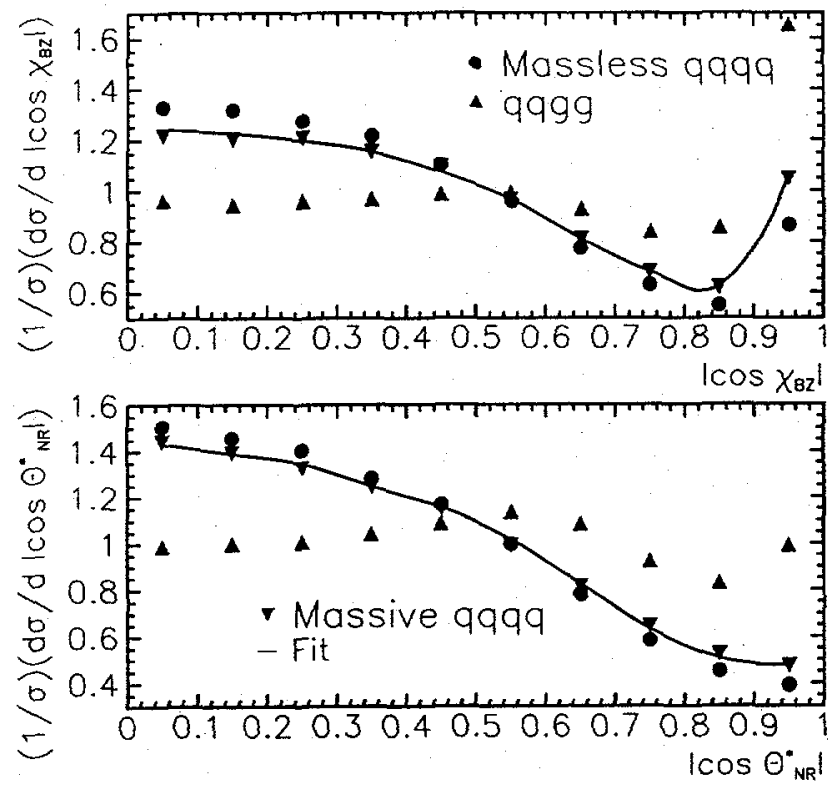

Figure 2.6: The distributions in BZ and NR angles of the $q \bar{q} q \bar{q}$ final state where the secondary quark has a mass of $5 \mathrm{GeV} / c^{2}$. They can be fit extremely well as a linear combination of massless $q \bar{q} q \bar{q}$ and $q \bar{q} g g$ distributions. $y_{\text {cut }}=0.03$ and $\sqrt{s}=m_{Z} c^{2}$ was assumed.

fits (which use BZ, NR and $\alpha_{34}$ angles simultaneously in a three-dimensional fit with 295 bins) quite well. Second, the confidence level is extremely flat up to $2 \mathrm{GeV} / c^{2}$. This implies that one does not need to worry about complication due to non-perturbative dynamics in defining the gluino mass. ${ }^{\S}$ The lower bound of ${ }^{\S}$ There are several possible definitions of gluino mass which may appear in experimental constraints: the mass of glueballino $m\left(R^{0}\right)$, the constituent mass $m_{\text {const }}(\tilde{g})$, the $\overline{\mathrm{MS}}$ current mass $\bar{m}_{\tilde{g}}\left(\bar{m}_{\tilde{g}}\right)$, the on-shell (pole) mass $m_{\text {pole }}(\tilde{g})$, and one half the mass of the pseudo-scalar gluinoball $m\left(\eta_{\tilde{g}}\right) / 2$. The various definitions are not expected to differ much from each other if $m_{\tilde{g}} \gtrsim 2 \mathrm{GeV} / c^{2}$. One may worry about this ambiguity for smaller gluino masses, but the result 


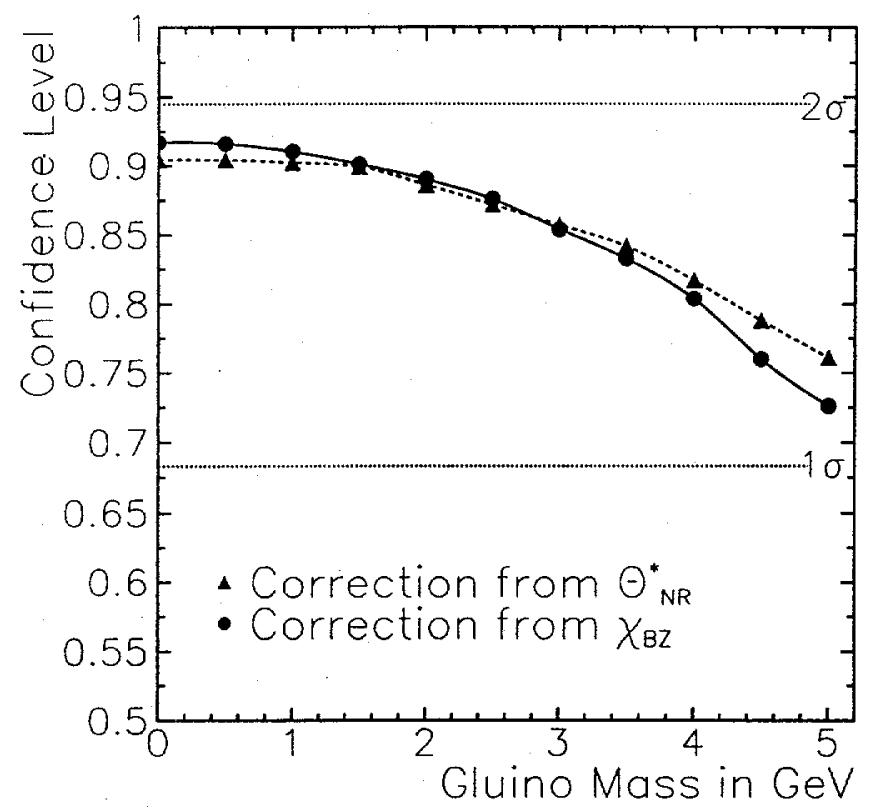

Figure 2.7: Exclusion confidence level of a light gluino as a function of its mass. Two curves are shown depending on the method of estimating the finite mass effects. In either case, a light gluino of mass below $1.5 \mathrm{GeV} / c^{2}$ is excluded at more than $90 \%$ confidence level.

$\simeq 1.5 \mathrm{GeV} / c^{2}$ at $90 \%$ confidence level is already in the perturbative region. It is quite likely that the gluino mass relevant to this analysis is a running mass defined at the scale $Q^{2} \sim y_{c u t} m_{Z}^{2}$. It is then straight-forward to convert the bound to the on-shell gluino mass: the lower bound of $\bar{m}_{\tilde{g}}\left(0.03 m_{Z}^{2}\right)=1.5 \mathrm{GeV} / c^{2}$ in the $\overline{\mathrm{MS}}$ scheme corresponds to $m_{\text {pole }}(\tilde{g})=2.8 \mathrm{GeV} / c^{2}$.

It is important to comment that the clever jet reconstruction method used presented here is insensitive to it since the confidence level in Fig. 2.7 is extremely flat up to $2 \mathrm{GeV} / c^{2}$. 
in the OPAL analysis [29] is particularly suited for the study of light gluinos in four-jet events. They did not scale the measured jet energies by an overall ratio $E_{v i s} / m_{Z}$, as done traditionally in similar analyses, but instead used the angular information of the jets to calculate the energy of each jet using energy and momentum conservation. This method avoids uncertainties in the gluino fragmentation. Since it is not well understood how a gluino fragments, one should use a similar method to avoid dependence on assumptions about the gluino fragmentation in future studies.

Unfortunately, the present analysis is limited to leading-order (LO) calculations. It is a natural question whether NLO corrections may change the present conclusions. First of all, one expects that the corrections to the angular variables used in the analysis are presumably not large. The NLO corrections are important when a variable involves $\alpha_{s}$, such as 3 - and 4-jet rates, thrust, etc. The variables used in the present analysis are not proportional to powers of $\alpha_{s}$, and hence scale-independent at the LO approximations. This is analogous to the case of the forward-backward asymmetry which is an (integrated) angular variable and is $\alpha_{s}$ independent at the LO. It does receive an NLO correction of $c\left(\alpha_{s} / \pi\right)$, where $c \sim 0.89$ in the case of a massless quark [53]. In the case at hand, one also expects a correction to the angular distributions of the order of $\alpha_{s}(\mu) / \pi$, where $\mu^{2} \sim y_{c u t} m_{Z}^{2}$ is probably an educated guess. Then a typical size of the NLO correction is about $5 \%$. However, a correction of this order of magnitude may still be of concern because of the following reason. The $q \bar{q} g g$ final state is roughly an 
order of magnitude larger than the $q \bar{q} q \bar{q}$ final state. Therefore, a $5 \%$ correction to $q \bar{q} g g$ may result in a $50 \%$ correction to $q \bar{q} q \bar{q}$ final state, to be compared with a possible $60 \%$ contribution from the gluino.

One may argue, however, that such a higher order correction is not likely to change the conclusion reached in this section. First of all, the helicity structure and the color flow in the $q \bar{q} g g$ final state and $q \bar{q} q \bar{q}$ final state are quite different. If a correction to the $q \bar{q} g g$ final state changes the conclusion, the following must be happening: the correction term to the $q \bar{q} g g$ exactly mimics an additional contribution to the $q \bar{q} q \bar{q}$ final state in the angular distributions with a negative sign such as to mask the contribution from the $q \bar{q} \tilde{g} \tilde{g}$ final state. This does not seem to be likely because they have different structures in the helicities and colors. Moreover, the data do not indicate that the NLO correction is large. OPAL data [29] are fit very well by the LO Monte Carlo on three-dimensional histograms of 295 bins with $\chi^{2} /$ d.o.f $=290 / 292$. This excellent agreement between the matrix element calculation and the data found in [29] supports the smallness of the NLO corrections empirically. However, the calculations of NLO corrections are necessary to justify it. ${ }^{\mathbb{T}}$ For future studies, it is also desirable to compare different Monte Carlo programs, while only JETSET was used in recent experimental papers [27, 28, 29].

Finally, it is worth emphasizing that the result in this section is based on

\footnotetext{
IIt is encouraging that partial NLO calculations were done after the completion of this work
} [54]. A preliminary study shows that the correction from leading terms in $1 / N_{c}^{2}$ expansion is small [55]. 
the 1991 and 1992 OPAL data with 1.1M hadronic $Z$ 's [29]. The statistical and systematic uncertainties are comparable in their paper. Given the current size of the LEP data, which is more than an order of magnitude larger, the statistical uncertainty should reduce substantially once all of the data has been analyzed. This change alone could drastically improve the sensitivity to the light gluino in four-jet events. On the other hand, it is not obvious how systematic uncertainties can be further reduced. The largest systematic uncertainty originates in the binby-bin acceptance corrections which needed to be done before performing a fit in $\mathrm{BZ}, \mathrm{NR}$, and the opening angle space. It is not clear how this uncertainty can be reduced if one employs the same method. Perhaps choosing larger values of $y_{\text {cut }}$ reduces the uncertainty while reducing the statistics at the same time. There could be an optimal choice of $y_{c u t}$ for this particular purpose. Some of the other large systematic uncertainties are specific to the OPAL experiment and could be reduced by averaging results from all four experiments. In any case, there is no doubt that a better result from the currently available data set can be expected.

In summary, the published OPAL 1991 and 1992 data on the QCD color factors [29] was reanalyzed in order to constrain possible additional contributions to fourjet events in $Z$ decays due to $q \bar{q} \tilde{g} \tilde{g}$ final states. The main difference from the original OPAL study is to fix $C_{A} / C_{F}=9 / 4$ as required by QCD. $T_{F} / C_{F} \geq$ $3 / 8$ is further imposed, and the finite mass effects of both the bottom quark and the gluino are carefully included. One finds that a light gluino with a mass below $1.5 \mathrm{GeV} / c^{2}$ is excluded at better than $90 \%$ confidence level. The result is 
insensitive to assumptions about what bound state it forms, the definition of its mass, and the gluino fragmentation provided that it does not decay inside the detectors. The currently available data set should be much more sensitive to a possible additional contribution from the light gluino." It is also argued that the NLO corrections are unlikely to modify the conclusion; still, this assertion needs to be justified by explicit calculations in the future. As a by-product of this analysis, the effect of finite bottom quark mass on $\mathrm{BZ}$ and NR distributions was discussed in detail, which turn out to be not negligible when extracting QCD color factors at current precisions.

\footnotetext{
"A paper by ALEPH [56] came out after the completion of this work, which claims to exclude a light gluino below $6.3 \mathrm{GeV}$ by combining the four-jet angular variables with the two-jet rate. This type of analysis may be more sensitive to the NLO corrections.
} 


\section{Chapter 3}

\section{Parameterizing the MSSM}

In this chapter, the "standard" parameterizations of the MSSM will be described. Furthermore, it will be shown that the phenomenology arising from these parameterizations is far too constrained, and a less constrained parameterization will be proposed. Some phenomenological implications of the proposed parameterization will be discussed.

\subsection{Introduction}

Supersymmetry (SUSY) is regarded as one of the most promising extensions of the Standard Model. A supersymmetric version of the Standard Model will be the subject of exhaustive searches in this and the next generation of collider experiments.

The Lagrangian of the minimal supersymmetric extension of the Standard Model, the so-called "Minimal Supersymmetric Standard Model" (MSSM), consists of a SUSY-preserving piece and a SUSY-breaking piece [57], as described in the previous chapter. The SUSY-preserving piece contains all of the Standard Model gauge and Yukawa couplings plus the so-called $\mu$-term, once $R$-parity is imposed to prevent baryon/lepton number violation. In this chapter, an exact or 
approximate $R$-parity is assumed, which implies that the lightest supersymmetric particle (LSP) does not decay inside detectors.

The SUSY-breaking Lagrangian will, ultimately, be determined by the physics of supersymmetry breaking and flavor but at the moment the best approach is to simply parameterize it with a general set of explicitly SUSY-breaking parameters. A general explicit soft SUSY-breaking Lagrangian

$$
\begin{aligned}
\mathcal{L}_{\mathrm{SUSYY}} & =-m_{H_{d}}^{2}\left|H_{d}\right|^{2}-m_{H_{u}}^{2}\left|H_{u}\right|^{2}+\left(B \mu H_{u} H_{d}+\text { H.c. }\right) \\
& -\left(\mathcal{A}_{d}^{i j} \tilde{Q}_{i} \tilde{d}_{j} H_{d}+\mathcal{A}_{u}^{i j} \tilde{Q}_{i} \tilde{u}_{j} H_{u}+\mathcal{A}_{l}^{i j} \tilde{L}_{i} \tilde{e}_{j} H_{d}+\text { H.c. }\right) \\
& -\sum_{F} m_{\tilde{F}}^{2 i j} \tilde{F}_{i}^{\dagger} \tilde{F}_{j}-\sum_{a=1,2,3}\left(M_{a} \lambda_{a} \lambda_{a}+\text { H.c. }\right)
\end{aligned}
$$

where $F=Q, L, u, d, e$ and $i, j=1,2,3$ for each generation, contains more than 100 new parameters and makes the study of the MSSM parameter space impractical. Furthermore, a random choice of SUSY-breaking parameters is most likely ruled out, because of flavor changing effects and CP-violation. In light of this situation, simplifying assumptions are not only welcome but necessary.

\subsection{The Standard Parameterizations}

In this section, the two most common parameterizations of the SUSY breaking sector of the MSSM will be described. They solve the experimental constraints mentioned above by imposing that most of the MSSM parameters vanish, and that there is a large degeneracy in the scalar mass-squared parameters. Both are inspired by SUSY breaking mechanisms, even though in the "hidden sector" or 
"minimal supergravity" models a very special form (which is, in general, hard to justify) for the high energy Lagrangian is required in order to yield the required scalar mass-squared degeneracy.

The "minimal supergravity" or "hidden sector" framework is the most commonly used set of assumptions imposed on the MSSM. In these models, SUSY is broken in a hidden sector and SUSY breaking effects are transmitted to the electroweak sector via nonrenormalizable operators, which are supressed by inverse powers of the Planck mass. Because it has nothing to do with supergravity itself, this framework will be referred to as the "Very Minimal Supersymmetric Standard Model" (VMSSM), to avoid confusion. It assumes a universal scalar mass-squared, gaugino mass, and trilinear coupling:

$$
\begin{gathered}
m_{\tilde{F}}^{2 i j}=m_{0}^{2} \delta^{i j} \text { for all } \tilde{F}, \\
m_{H_{u}}^{2}=m_{H_{d}}^{2}=m_{0}^{2}, \\
M_{a}=M_{1 / 2} \text { for all } a, \\
\mathcal{A}_{f}^{i j}=A_{0} \lambda_{f}^{i j} \text { for all } f,
\end{gathered}
$$

where $\lambda_{f}^{i j}$ are the ordinary Yukawa couplings, at the grand unified (GUT) scale. The VMSSM is, therefore, parameterized by five real parameters: $m_{0}^{2}, M_{1 / 2}, A_{0}$, $\mu$, and $B$ [58].

More recently a lot of work has been done on models with the gauge mediation of SUSY breaking (GMSB) [59]. In these models, SUSY is broken in a secluded sector, and the effects of SUSY breaking are mediated to the electroweak sector 
via gauge interaction loop effects. More will be said about these models in future chapters. For now, the only relevant information one needs to extract from these models is that the MSSM soft SUSY breaking parameters are predicted to be, at the messenger scale $M$,

$$
\begin{gathered}
M_{a}=\frac{\alpha_{a}}{4 \pi} N \frac{F}{M}, \\
m_{\tilde{F}}^{2 i j}=m_{\tilde{F}}^{2} \delta^{i j}, \\
m_{\tilde{F}}^{2}=2 N\left(\frac{F}{M}\right)^{2}\left(\frac{3}{5} Y_{\tilde{F}}^{2}\left(\frac{\alpha_{1}}{4 \pi}\right)^{2}+C_{2 \tilde{F}}\left(\frac{\alpha_{2}}{4 \pi}\right)^{2}+C_{3 \tilde{F}}\left(\frac{\alpha_{3}}{4 \pi}\right)^{2}\right), \\
\mathcal{A}_{\tilde{f}}^{i j}=0 \text { for all } f,
\end{gathered}
$$

where $\alpha_{a}=g_{a}^{2} / 4 \pi$ and $g_{a}$ are the gauge coupling constants in the $S U(5)$ normalization. $Y_{\tilde{F}}$ is the hypercharge of the $\tilde{F}=\tilde{Q}, \tilde{u}, \tilde{d}, \tilde{L}, \tilde{e}, H_{u}, H_{d}$ scalars, $C_{2 \tilde{F}}=3 / 4$ for weak $S U(2)$ doublets (zero for singlets) and $C_{3 \tilde{F}}=4 / 3$ for color triplets (zero for color singlets). Again, just five real parameters are introduced: $F / M, M, N, \mu$, and $B$. Note that Eq. (3.2.2) guarantees that squarks of different families are degenerate at the messenger scale and therefore FCNC effects are safely suppressed. The fact that FCNC are naturally suppressed is one of the main motivations for models with the GMSB [59].

It is important to note that the particle spectra of models with the GMSB are similar to those of the VMSSM* and, therefore, the remainder of this chapter will concentrate on the VMSSM and possible modifications to it.

*The major difference from the VMSSM is that the gravitino $(\tilde{G})$ is most likely the LSP. This can lead, e.g., to photonic signatures from the decay $\tilde{\chi}_{1}^{0} \rightarrow \gamma \tilde{G}$. 


\subsection{Consequences of the Minimal Parameterization}

The issue which is important to address is how restrictive the VMSSM is to collider phenomenology. It is important to be able to explore diverse particle spectra while still satisfying all experimental bounds and keeping the number of parameters small, and the fact that the VMSSM has only a handful of parameters (instead of over 100) is of some concern. In this section the issue of how experimental constraits restrict the VMSSM will be addressed.

Only constraints from particle physics will be considered throughout the chapter. In our opinion, it is not wise to impose any cosmological constraints on the parameter space for the experimental analysis of collider data. Even though cosmology does provide many useful constraints on parameters of particle physics, cosmology at temperatures between the electroweak scale and nucleosynthesis may be much more complex than usually assumed. For instance, most models of SUSY breaking create cosmological problems, which can be avoided only by invoking inflation at temperatures below the electroweak scale [60]. Such a drastic change removes the constraints that the LSP must be neutral and should not overclose the Universe. Very small $R$-parity violating couplings can also evade the cosmological constraints without any consequences to collider phenomenology [61]. In light of this discussion, the parameter space should be explored without much theoretical prejudice.

First, the VMSSM parameter space and spectrum will be briefly reviewed. 
The soft SUSY-breaking parameters at the weak scale are found by solving the renormalization group $(\mathrm{RG})$ equations [62], which are quoted in Appendix $\mathrm{F}$. In Table 3.1 the results of numerically running the 1-loop RG equations from the GUT scale down to $500 \mathrm{GeV}$ as a function of $m_{0}^{2}, M_{1 / 2}$, and $A_{0}$, for $\tan \beta=10$ are quoted as an example. The parameters $\mu$ and $B$ run "by themselves", and one can, therefore, specify their input values at the weak scale.

It is necessary to check that the electroweak symmetry has been broken and that $M_{Z}=91 \mathrm{GeV}$. This is done by choosing $\mu^{2}$ such that

$$
\mu^{2}=-\frac{M_{Z}^{2}}{2}+\frac{m_{H_{d}}^{2}-m_{H_{u}}^{2} \tan ^{2} \beta}{\tan ^{2} \beta-1},
$$

where $\tan \beta$ is the ratio of Higgs boson vacuum expectation values, $v_{u} / v_{d}$. This is, of course, a tree-level result, which is known to receive corrections of order a few percent [63] at one-loop. In order to keep the presentation simple, however, only use the tree-level results will be used. Another condition which must be satisfied involves the $B$-term. Once $\tan \beta$ is specified, the $B$-parameter is uniquely determined and is related to the pseudoscalar Higgs mass squared,

$$
m_{A}^{2}=m_{H_{d}}^{2}+m_{H_{u}}^{2}+2 \mu^{2}=2 \frac{B \mu}{\sin (2 \beta)} .
$$

To prevent a runaway behavior in the Higgs scalar potential $m_{A}^{2}$ must be positive. After imposing Eqs. (3.3.1,3.3.2), the VMSSM contains only four extra real free parameters: $m_{0}^{2}, M_{1 / 2}, A_{0}, \tan \beta$, plus a discrete choice, $\operatorname{sign}(\mu)$.

Table 3.1 indicates the structure of the particle spectrum: colored sparticles are heavier than sparticles that only transform under $S U(2)_{L} \times U(1)_{Y}$ which in 
Table 3.1: SUSY-breaking parameters at a scale of $500 \mathrm{GeV}$ from the 1-loop RG equations with the VMSSM boundary conditions at $M_{G U T}=1.86 \times 10^{16} \mathrm{GeV}$, for (A) the first/second generation sfermions and (B) the rest with $\tan \beta=10$. The masses of first/second generation fermions have been neglected, and $h_{t}\left(m_{t}\right)=$ $165 /(174 \sin \beta)$ was used. The table is to be read as follows: each soft parameter is a linear combination of the input parameters, with the coefficients given in the table. For example, $m_{H_{d}}^{2}=0.95 m_{0}^{2}+0.38\left(M_{1 / 2}\right)^{2}-0.01\left(A_{0}\right)^{2}-0.04 M_{1 / 2} A_{0}-1 / 2 D_{Y}$ and $A_{\tilde{d}}=A_{0}+3.41 M_{1 / 2}$.

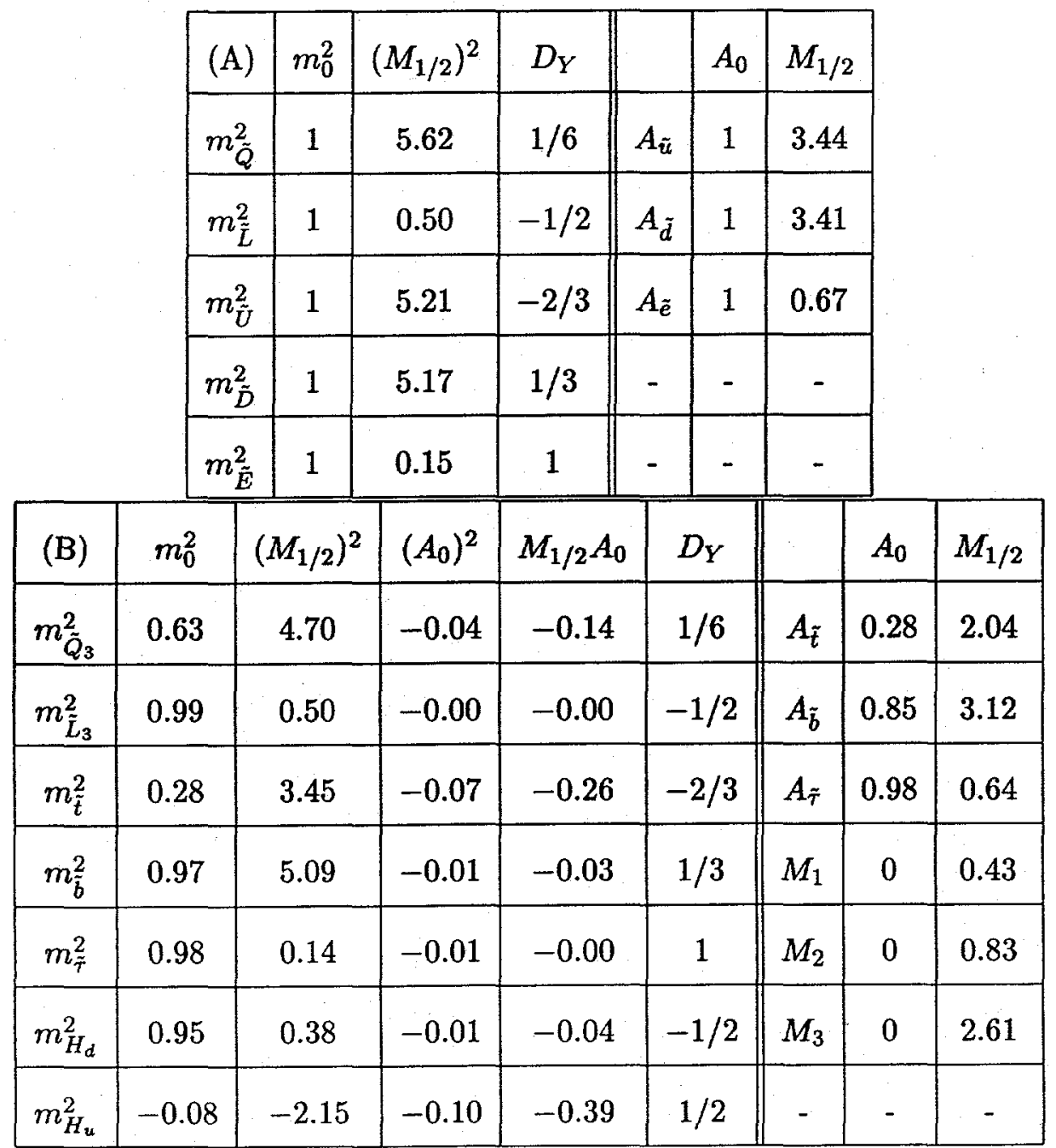


turn are heavier than those that only transform under $U(1)_{Y}$. Furthermore $\mu^{2}$ can be numerically evaluated with the help of Eq. (3.3.1),

$$
\begin{aligned}
\mu^{2} & =2.18\left(M_{1 / 2}\right)^{2}+0.09 m_{0}^{2}+0.10\left(A_{0}\right)^{2}+ \\
& +0.39 M_{1 / 2} A_{0}-\frac{1}{2} M_{Z}^{2}
\end{aligned}
$$

for $\tan \beta=10$. From gluino searches, $M_{1 / 2} \gtrsim 77 \mathrm{GeV}$ (for $M_{3} \gtrsim 200 \mathrm{GeV}$ ), and therefore $\mu^{2} \gtrsim 2.14 M_{2}^{2}$. It is then safe to say that the lightest neutralino is an almost pure B-ino of mass $m_{\tilde{\chi}_{1}^{0}} \simeq M_{1}[64]$.

There are two LSP candidates: the right-handed scalar tau $\left(\tilde{\tau}_{R}\right)$ and the lightest neutralino $\left(\tilde{\chi}_{1}^{0}\right)$. It is easy to see that $\tilde{\chi}_{1}^{0}$ is always the LSP unless $m_{0}^{2} \lesssim\left(0.04 M_{1 / 2}^{2}-\right.$ 1890) $(\mathrm{GeV})^{2}$, for $\tan \beta=10$. In theories with the GMSB one can actually have a $\tilde{\tau}_{R}$ LSP for a larger portion of the parameter space if the number of messengers $(N)$ is large enough [59].

\subsection{A Less Constrained Parameterization}

In this section a "Less Minimal Supersymmetric Standard Model" (LMSSM) is proposed, which adds only one extra parameter to the VMSSM: the FayetIliopoulos $D$-term for the $U(1)_{Y}$ gauge group, $D_{Y}$. Unlike the VMSSM, this framework will prove to be general enough to allow the following additional phenomenological possibilities: a stable charged slepton, a higgsino-like neutralino, or a sneutrino as the LSP. Different particle spectra result in very different decay patterns, lifetimes and branching ratios which lead to different signals for SUSY 
searches, as will be discussed later.

A Fayet-Iliopoulos $D$-term for the $U(1)_{Y}$ gauge group is indeed generated in many interesting theoretical scenarios. A kinetic mixing between $U(1)_{Y}$ and a different $U(1)$ can induce a $D$-term once the other $U(1)$ develops a $D$-component vacuum expectation value [65]. The other $U(1)$ can be a part of the gauge group responsible for dynamical SUSY breaking, or an anomalous $U(1)$ in superstring theory whose anomaly is canceled by the Green-Schwarz mechanism. In models with the GMSB it can also be the messenger $U(1)$ [66]. The goal of this section is, however, to study the effect of the parameter $D_{Y}$ on phenomenology, therefore, its origin will not be discuused any further.

In the LMSSM the Fayet-Iliopoulos $D$-term $\left(D_{Y}\right)$ changes the mass squared parameters of all the scalars to $m_{\tilde{F}}^{2}=m_{\tilde{F}, V}^{2}+Y_{\tilde{F}} D_{Y}$ at some energy scale, where the subscript $V$ stands for VMSSM and $Y_{\tilde{F}}$ is the hypercharge of the scalar $\tilde{F}$. Note that $Y_{\tilde{F}} D_{Y}$ is flavor-blind and, therefore, the flavor-changing constraints are safely avoided.

There is one very important simplification which is peculiar to the parameter $D_{Y} . D_{Y}$ runs by itself and hence it does not matter at what energy scale the scalar mass-squared parameters are modified. Therefore, it is convenient to calculate $m_{\tilde{F}, V}^{2}$ at the weak scale from the inputs $m_{0}^{2}, M_{1 / 2}$, and $A_{0}$ (see Table 3.1 ) and add the weak-scale value of $Y_{\tilde{F}} D_{Y}$.

Similarly to the VMSSM, electroweak symmetry breaking imposes constraints 
on the parameter space. One way to satisfy Eq. (3.3.1) is to choose $D_{Y}$ such that

$$
\frac{M_{Z}^{2}}{2}+\mu^{2}-\frac{m_{H_{d}, V}^{2}-m_{H_{u}, V}^{2} \tan ^{2} \beta}{\tan ^{2} \beta-1}=\frac{D_{Y}}{2 \cos 2 \beta}
$$

Note that the form of Eq. (3.3.2) is unchanged. The free parameters are, therefore,

$$
m_{0}^{2}, M_{1 / 2}, A_{0}, \tan \beta, \text { and } \mu .
$$

Unlike in the VMSSM, $\mu$ is a free parameter in the LMSSM. It does not, for example, have to be larger than $M_{2}$ or even $M_{1}$. This will change phenomenology drastically. Note that exactly the same strategy can be followed to add $D_{Y}$ as an extra parameter to models with the GMSB. Again, it is important to note that the one-loop effective potential changes the value of $D_{Y}$ extracted from Eq. (3.4.1) and, therefore, the plots presented in Figs. 3.1 and 3.2 will be somewhat distorted. ${ }^{\dagger}$ However, none of the qualitative features of the parameterization are lost.

Varying $D_{Y}$ (or $\mu$ ) affects different parameters in different ways. For negative $D_{Y}, \tilde{e}_{i}, \tilde{d}_{i}$, and $\tilde{Q}_{i}\left(i\right.$ is a generation index) become lighter (the effect on $m_{\tilde{d}}^{2}$ and $m_{\tilde{Q}}^{2}$ is, however, small because of their hypercharges), while other sfermions become heavier. In this case the absolute value of the $\mu$-term is larger than in the VMSSM (see Eq. (3.4.1)). If $D_{Y}$ is large enough compared to $M_{1 / 2}, \tilde{\tau}_{R}$ becomes the LSP. Note that, unlike in the VMSSM, this happens for a large range of values of $m_{0}^{2}$. Fig. 3.1 depicts the nature of the LSP in the $\left(\mu, M_{1 / 2}\right)$ plane for fixed values of

${ }^{\dagger}$ We have numerically verified that the extracted value of $D_{Y}$ changes only by $10-20 \%$ when the full one-loop effective Higgs potential is taken into account. For a complete discussion of one-loop effects in the VMSSM spectrum see, e.g., [63]. 


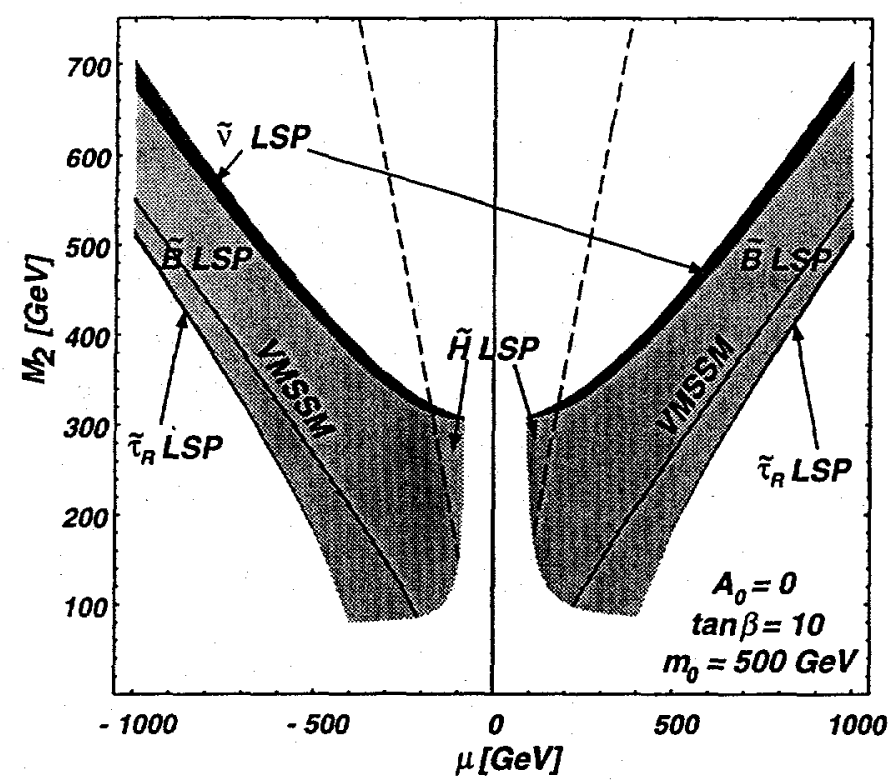

Figure 3.1: Parameter space analysis indicating the nature of the LSP. The solid line indicates the points allowed by the VMSSM and the dashed line represents points where the gaugino content of $\tilde{\chi}_{1}^{0}$ is $50 \% . A_{0}=0, m_{0}^{2}=500^{2}(\mathrm{GeV})^{2}$ and $\tan \beta=10$. The bounds $m_{A}>65 \mathrm{GeV}, m_{\tilde{\nu}}>43 \mathrm{GeV}, m_{\tilde{\tau}}>67 \mathrm{GeV}$ (if $\left.m_{\tilde{\tau}}<m_{\tilde{\chi}_{1}^{0}}\right)$, and $m_{\tilde{\chi}_{1}^{ \pm}}>65 \mathrm{GeV}$ were imposed.

$m_{0}^{2}$ and $\tan \beta$. For smaller (larger) values of $m_{0}^{2}$ or larger (smaller) values of $\tan \beta$, the size of the physically allowed region decreases (increases), but the qualitative features of the figure remain the same (with the exception of large $\tan \beta \gtrsim 30$, see below). See Fig. 3.2.

For positive $D_{Y}, \tilde{L}_{i}$ and $\tilde{u}_{i}$ become lighter, while all other sfermion masses increase. In this case the absolute value of $\mu$ is smaller than in the VMSSM. The consequences of this are many (see Fig. 3.1). $\tilde{\nu}_{\tau}$ can become the LSP. If $\mu$ is small enough, $\tilde{\chi}_{1}^{0}$ can be the LSP but with a large higgsino content. The mass splitting 


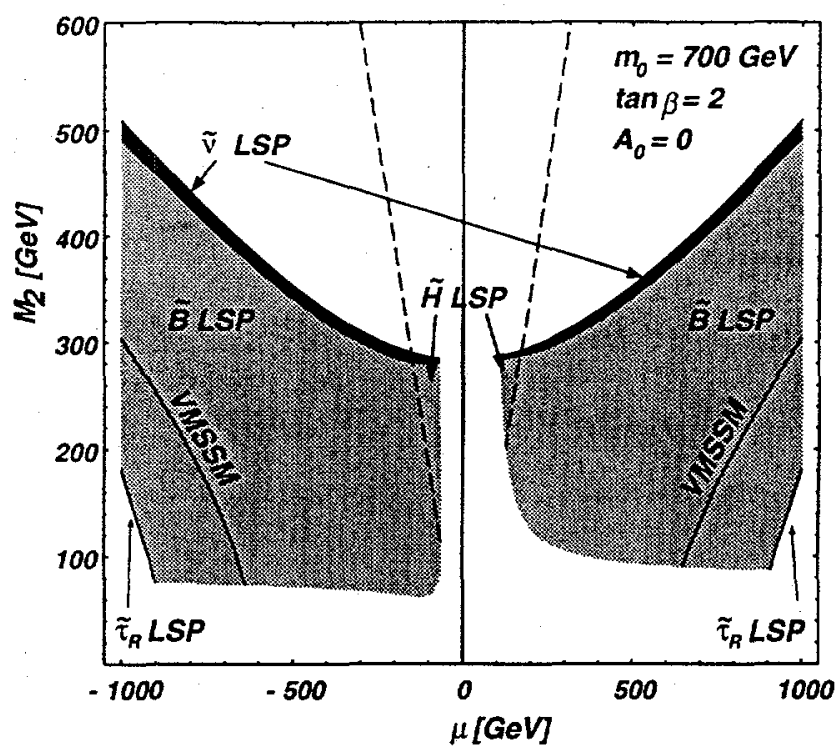

Figure 3.2: Same as Fig. 3.1, for $A_{0}=0, m_{0}^{2}=700^{2}(\mathrm{GeV})^{2}$ and $\tan \beta=2$.

between $\tilde{t}^{\prime}$ s is enhanced with respect to the VMSSM. Finally, if $\tan \beta \gtrsim 30$ and $\mu$ is large, the left-handed $\tilde{\tau}$ can become the LSP due to left-right mixing in the mass squared matrix.

We would like to draw attention to the existence of different particle spectra for different regions in the parameter space rather than the size of those regions (see Fig. 3.1). Like the VMSSM, the LMSSM should be considered as a parameterization and not a model, and the fact that diverse spectra can occur is what is of interest.

\subsection{Some Phenomenological Consequences}

In this section, interesting aspects of the phenomenology of the spectra outlined above are discussed semi-quantitatively. 
If $\tilde{\tau}$ is the LSP, heavy stable charged particles become a good signature for SUSY searches. An analysis of this situation was done in the context of models with the GMSB where the $\tilde{\tau}_{R}$ is the LSP [67]. Heavy stable charged particles might be found by looking for an excess of hits in the muon chambers, or tracks with anomalously large $d E / d x$ in the tracking chambers.

If the LSP is a higgsino-like neutralino, the phenomenology is very different from the VMSSM case, where the LSP is an almost pure B-ino [68]. In this case there are four fermions relatively close in mass: $\tilde{\chi}_{1}^{0}, \tilde{\chi}_{2}^{0}$ and $\tilde{\chi}_{1}^{ \pm}$, which are all higgsino-like. In this situation experimental searches are much harder. Chargino searches become more difficult because the mass splitting between $\tilde{\chi}_{1}^{ \pm}$and $\tilde{\chi}_{1}^{0}$ becomes very small $\left(m_{\tilde{\chi}_{1}^{ \pm}}-m_{\tilde{\chi}_{1}^{0}} \simeq m_{W}^{2} / M_{1 / 2}\right.$ in the limit of $\left.M_{2} \gg \mu, m_{W}\right)$, and $\tilde{\chi}_{1}^{ \pm}$will decay into missing transverse energy $\left(\not_{T}\right)$ plus low energy leptons or jets $\left(E_{l, j} \simeq 6 \mathrm{GeV}\right.$ if $\left.M_{1 / 2}=600 \mathrm{GeV}\right)$. Experimental searches for chargino signals at the Tevatron usually require that $E_{T}^{l, j}>15 \mathrm{GeV}[58] .{ }^{\ddagger}$

At hadron machines the amount of $E_{T}$ is reduced because of the small coupling between first and second generation squarks and $\tilde{\chi}_{1}^{0, \pm}$. The main decay mode of a squark is $\tilde{q} \rightarrow q \tilde{\chi}_{3,4}^{0}$ or $q^{\prime} \tilde{\chi}_{2}^{ \pm}$, and the heavier chargino/neutralinos, which are gaugino-like, further decay via, e.g., $\tilde{\chi}_{2}^{ \pm} \rightarrow \tilde{\chi}_{1}^{0} H^{ \pm}$. The decay chains are therefore much longer and the amount of $E_{T}$ should decrease. It is interesting to note that there might be a significant increase in the number of top quark, b-jet, and $\tau$

\footnotetext{
${ }^{\ddagger}$ If the splitting between $\tilde{\chi}_{1}^{ \pm}$and $\tilde{\chi}_{1}^{0}$ is small enough, i.e. $\Delta m \lesssim 1 \mathrm{GeV}$, the chargino will decay with a displaced vertex. This, however, requires that $M_{1 / 2} \gtrsim 6 \mathrm{TeV}$.
} 
events because of the production of heavy Higgs boson states $\left(H^{0, \pm}, A^{0}\right)$, which have large branching ratios into third generation fermions.

The clean tri-lepton signature at hadron machines will decrease by an order of magnitude mainly because of the smaller leptonic branching ratio for $\tilde{\chi}_{2}^{0}$ and $\tilde{\chi}_{1}^{ \pm}$. Note that this effect is not restricted to the pure higgsino-like neutralino limit, but also applies to a mixed $\tilde{\chi}_{1}^{0}[58]$.

If the LSP is $\tilde{\nu}_{\tau}$, the decay modes of the heavier particles change dramatically. There are different possibilities, depending on $m_{\tilde{l}}$ and $m_{\tilde{\chi}_{1}^{0}}$.

If $m_{\tilde{l}}<m_{\tilde{\chi}_{1}^{0}}$ the main decay mode for sleptons is $\tilde{l} \rightarrow \tilde{\nu} j j$ or $\tilde{l} \rightarrow \tilde{\nu} l^{\prime} \nu_{l^{\prime}}$. Charginos, on the other hand, decay into two particles, namely $\tilde{\chi}^{ \pm} \rightarrow \tilde{\nu} l$ or $\rightarrow \tilde{l} \nu$. The pair production of two sleptons at an $e^{+} e^{-}$machine will yield, for instance, $l j j \not E$, which is the typical chargino pair production signal in the VMSSM. The production of a chargino-pair will yield acoplanar leptons plus $E$, which is the typical slepton signal at $e^{+} e^{-}$machines in the VMSSM. The two leptons, however, do not have to be of the same flavor. There are, of course, ways of distinguishing a slepton signature in the VMSSM from the chargino signal in this scenario because the cross sections and angular distributions are quite different.

Another important feature is the visible decay $\tilde{\chi}_{1}^{0} \rightarrow \tilde{l} l$. This makes the production $q \bar{q} \rightarrow \tilde{\chi}_{1}^{0} \tilde{\chi}_{1}^{0}$ a feasible SUSY signature. Furthermore squarks decay dominantly as $\tilde{q} \rightarrow q \tilde{\chi}_{1}^{0}$ because $\tilde{u}_{i}$ are much lighter than $\tilde{Q}_{i}$ or $\tilde{d}_{i}$, and hence the squarks produced are dominantly $\tilde{u}_{i}$. This can lead to a rather impressive four leptons plus jets plus $\not_{T}$ signature at hadron machines. The total fraction of $4 l$ events is only 
about $0.5 \%$ because typically $B R\left(\tilde{\chi}_{1}^{0} \rightarrow \tilde{l}\right) \simeq 1 / 3$ and $B R\left(\tilde{l} \rightarrow \tilde{\nu}_{l} \bar{l}^{\prime} \nu_{l^{\prime}}\right) \simeq 20 \%$ for $l, l^{\prime}=e$ or $\mu$, but they have much lower backgrounds [58].

In the case $m_{\tilde{l}}>m_{\tilde{\chi}_{1}^{0}}$ both the $\tilde{\chi}_{1}^{ \pm}$and the $\tilde{l}$ decay into two on-shell particles $\left(\tilde{l} \rightarrow \tilde{\chi}_{1}^{0} l\right)$. The $\tilde{\chi}_{1}^{0}$, though unstable, is still invisible, because its only allowed decay mode is $\tilde{\chi}_{1}^{0} \rightarrow \nu \tilde{\nu}$. This scenario has, therefore, four "virtual LSPs" (3 $\tilde{\nu}$ and the $\left.\tilde{\chi}_{1}^{0}\right)$. In this case the amount of $\not_{T}$ in hadron machines is virtually unchanged with respect to the VMSSM [69]. Note that the clean tri-lepton signature is enhanced (given that $\tilde{\chi}_{2}^{0} \rightarrow l \tilde{l}$ is allowed with reasonable branching ratio) because both the $\tilde{\chi}_{1}^{ \pm}$and the $\tilde{l}$ always decay into one charged lepton.

Finally, there is another type of signature, which has no VMSSM analog, if the sneutrino is the LSP and $\tan \beta \gtrsim 4$ : visible sneutrino decays, $\tilde{\nu}_{l} \rightarrow l^{-} \tau^{+} \tilde{\nu}_{\tau}$. In this case the first and second generation sneutrinos are heavier than $\tilde{\nu}_{\tau}$ enough to decay visibly. The other allowed sneutrino decays are $\tilde{\nu}_{l} \rightarrow \nu_{l} \tilde{\nu}_{\tau} \bar{\nu}_{\tau}$ and $\tilde{\nu}_{l} \rightarrow \nu_{l} \tilde{\nu}_{\tau}^{*} \nu_{\tau}$. For $\tan \beta=10, m_{0}^{2}=500^{2}(\mathrm{GeV})^{2}, m_{\tilde{\nu}_{\tau}}=75 \mathrm{GeV}$ and $M_{1}=185 \mathrm{GeV}, \Delta m \simeq 15 \mathrm{GeV}$ and the visible branching ratio is approximately $7 \%$. In this scenario, there is a very striking signature for $\tilde{\nu}_{l} \tilde{\nu}_{l}^{*}(l=\mu, e)$ production in $e^{+} e^{-}$machines if one of the sneutrinos decays visibly and the other invisibly. One expects to see $l^{ \pm} \tau^{\mp}$ plus $\not_{T}$ for $2 \times(.07 \times .93)=13 \%$ of all $\tilde{\nu}_{l} \tilde{\nu}_{l}^{*}$ produced, for the parameters mentioned earlier. It is important to note that, for larger $\tan (\beta), \Delta m$ can be significantly larger, and therefore both the $\tau$ and the $l$ are easily visible at LEP (for $\tan \beta \simeq 16$, $\Delta m \simeq 35 \mathrm{GeV})$. The main backgrounds for this signal are $e^{+} e^{-} \rightarrow W^{+} W^{-}$and $\gamma \gamma \rightarrow \tau^{+} \tau^{-}$. However, simple kinematic cuts should efficiently suppress these 
events, because their kinematics are quite different from the signal's. A systematic study of the appropriate cuts is beyond the scope of this chapter. There is also the possibility that $\tilde{\nu}_{l}$ decays with a displaced vertex, if $\Delta m$ is small enough. In this case, however, the visible branching ratio is significantly smaller because of the phase space reduction due to the tau mass.

\subsection{Summary}

In summary, the standard parameterizations of the MSSM, as far as collider phenomenology is concerned, were described. It was shown that the so-called "Minimal Supergravity Inspired" Supersymmetric Standard Model is too restrictive as far as collider phenomenology is concerned. The addition of only one extra parameter to the VMSSM, the Fayet-Iliopoulos $D$-term for $U(1)_{Y}$, was proposed and it was shown that it is capable of yielding a much more diverse phenomenology while still satisfying all experimental constraints.

While the VMSSM almost always yields a B-ino-like LSP, the LMSSM also allows $\tilde{\nu}, \tilde{\tau}$ or Higgsino-like $\tilde{\chi}_{1}^{0}$ LSP. It was verified that for each one of these cases there are important phenomenological consequences, including new signatures for SUSY and the disappearance of other "standard" signatures. Even though the LMSSM is not advocted as the model of SUSY breaking, it is important to emphasize that it is a much less restrictive, and yet workable, parameterization of the SUSY breaking sector. 


\section{Chapter 4}

\section{Cosmology of Models with Gauge Mediated}

\section{SUSY Breaking}

\subsection{Introduction}

The promise of low-energy supersymmetry (SUSY) is to stabilize the hierarchy between the weak scale and a higher scale of new physics, e.g. the Planck scale (see, e.g., [70]). SUSY, however, has to be spontaneously broken because we do not see degenerate pairs of particles and their superpartners. Moreover, there are stringent phenomenological constraints on the spectrum of superparticles such as the degeneracy among squarks or sleptons at the percent level (see, e.g., [71]). Therefore, constructing viable mechanisms of SUSY breaking has been regarded as one of the most important issues in SUSY model building.

Recently, the idea of generating SUSY-breaking masses via gauge interactions has attracted interests (Low-Energy Gauge Mediation, or LEGM) [72, 73, 74]. In this scheme, the SUSY breaking effects appear in the supersymmetric Standard Model in the following manner. There is a sector which breaks SUSY dynamically at around a $10^{7} \mathrm{GeV}$ scale, and it generates SUSY breaking effects in the socalled messenger sector at around $10^{5} \mathrm{GeV}$, which further induce SUSY breaking 
masses of order $10^{3} \mathrm{GeV}$ in the supersymmetric Standard Model via ordinary gauge interactions. This mechanism guarantees the required degeneracy among squarks, sleptons at a high degree, and also generates the masses for scalars and gauginos at comparable magnitudes as desired phenomenologically. There have been active studies on the phenomenology of such models $[75,76]$. On the other hand, there has been little discussion on cosmological consequences of this mechanism, except issues concerning stable particles in the messenger sector [77]. Since the scheme completely differs from the conventional hidden sector scenario at high energies, early cosmology is expected to differ substantially as well.

There are (at least) two ingredients in the LEGM models which may lead to a cosmology different from the hidden sector case. The first is a very small gravitino mass. Since SUSY is broken at around $10^{7} \mathrm{GeV}$, compared to around $10^{10} \mathrm{GeV}$ in the hidden sector scenario, the gravitino mass is much lighter: $m_{3 / 2} \sim 100 \mathrm{keV}$ compared to $100 \mathrm{GeV}$. The second is that the SUSY breaking effects "shut off" at high energies.* In particular, the flat directions in the supersymmetric Standard Model have very different potentials at large field amplitudes.

In this chapter, the implications of the LEGM models to cosmology are studied. In Sec. 4.2, the cosmological constraints on light gravitinos mainly based on the analysis by Moroi, Murayama and Yamaguchi [79], is firts discussed. Then, in Sec. 4.3, particular attention is paid to the estimate of the gravitino mass in the LEGM models, and it is argued that it is highly unlikely to be lighter than $2 h^{2} \mathrm{keV}$

\footnotetext{
*A similar effect was discussed in [78] in the context of the sliding singlet mechanism.
} 
as required by cosmology. This point implies that there must be a substantial entropy production, which casts a concern on the baryon asymmetry. Therefore, the attention will be turned to a possible mechanism of baryogenesis at a relatively low temperature, using the idea of Affleck and Dine [80]. The important point in the LEGM models is that the SUSY breaking effects due to the messenger interaction "shut off" at high energies. Therefore, in Sec. 4.4, a 2-loop calculation to determine the shape of the potential for the flat direction was performed. In Sec. 4.5 , the possible value of the baryon-to-entropy ratio which can be induced by the Affleck-Dine baryogenesis is estimated. For a sufficiently large amplitude of the flat direction, the potential is dominated by the supergravity contribution rather than the LEGM contribution, and one will see that the Affleck-Dine baryogenesis works well enough to explain the present value of the baryon asymmetry. Furthermore, in Sec. 4.6, it is pointed out that the string moduli, if present within the LEGM models, cause a serious problem because they are stable and their coherent oscillations grossly overclose the Universe. However, a possible solution to the problem is also shown. Since the Affleck-Dine baryogenesis is so efficient, the baryon asymmetry can survive the enormous entropy production required to dilute the moduli fields, possibly by thermal inflation [81]. Finally, Sec. 4.7, contains a summary and some conclusions.

\subsection{Cosmology of a Light Gravitino}

In this section, a brief review of the cosmology with a light stable gravitino [79] 
is given. ${ }^{*}$ If a stable gravitino is thermalized in the early Universe, and if it is not diluted by some mechanism (such as a late inflation and/or a substantial entropy production), its mass density may exceed the closure limit: $\Omega_{3 / 2}<1$. Since the number density of the gravitino is fixed once the gravitinos are thermalized, the above argument sets an upper bound on the gravitino mass [82]:

$$
m_{3 / 2} \lesssim 2 h^{2} \mathrm{keV}: \text { without dilution, }
$$

where $h$ is the Hubble constant in units of $100 \mathrm{~km} / \mathrm{sec} / \mathrm{Mpc}$. In other words, if the gravitino mass is heavier than $2 h^{2} \mathrm{keV}$, some mechanism is necessary to dilute the gravitino in order not to overclose the Universe. Since the gravitinos are produced more at a higher temperature, one can obtain an upper bound on the maximal temperature, $T_{\max }$, from which the ordinary radiation dominated Universe starts. For example, in the inflationary Universe, $T_{\max }$ corresponds to the so-called reheating temperature $T_{\mathrm{RH}}$ which is typically higher than $T_{\mathrm{RH}} \gtrsim 10^{8}$ $\mathrm{GeV}$, if there is no significant entropy production after reheating. If $T_{\max }$ turns out to be less than $10^{8} \mathrm{GeV}$ or so, we judge that one needs a substantial entropy production below $T_{\mathrm{RH}}$. It is worthwhile to recall that the recent measurements prefer $h \sim 0.7$ and hence the upper bound is about $1 \mathrm{keV}$.

The crucial point about the light gravitino is that the interaction of the (longitudinal component of) gravitino becomes stronger as the gravitino mass gets lighter. This is because the longitudinal component of the gravitino behaves like the goldstino, whose interaction is proportional to $\langle F\rangle^{-1} \sim\left(m_{3 / 2} M_{*}\right)^{-1}$, where

\footnotetext{
*In this chapter, the absolute conservation of $R$-parity is assumed.
} 
$M_{*}=2.4 \times 10^{18} \mathrm{GeV}$ is the reduced Planck scale. For the light gravitino, the interaction of the longitudinal component of the gravitino ( $\sim$ the goldstino) $\psi$ to the chiral multiplet $(\phi, \chi)$ and to the gauge multiplet $\left(A_{\mu}, \lambda\right)$ is given by [83]

$$
\mathcal{L}=\frac{i m_{\lambda}}{8 \sqrt{6} m_{3 / 2} M_{*}} \bar{\psi}\left[\gamma_{\mu} \gamma_{\nu}\right] \lambda F^{\mu \nu}+\frac{m_{\chi}^{2}-m_{\phi}^{2}}{\sqrt{3} m_{3 / 2} M_{*}}\left(\bar{\psi} \chi_{\mathrm{L}}\right) \phi^{*}+\text { h.c. }
$$

where $m_{\phi}, m_{\chi}$, and $m_{\lambda}$ represent the masses of $\phi, \chi$, and $\lambda .^{\dagger}$ As indicated in Eq. (4.2.2), the interaction of $\psi$ becomes stronger as the gravitino mass gets smaller.

In the thermal bath, two types of the processes may contribute to overproduce the gravitino: one is the decay of the sparticle $\tilde{X}$ into its superpartner $X$ and the gravitino, $\tilde{X} \rightarrow \dot{\psi}+X$, and the other is the scattering processes, $x+y \rightarrow \psi+z$, where $x, y, z$ are relevant (s)particles. The decay process is significant especially for the case $m_{3 / 2} \lesssim 100 \mathrm{keV}$. The partial decay width of a sparticle $\tilde{X}$ into the gravitino is estimated as

$$
\Gamma(\tilde{X} \rightarrow \psi+X) \sim \frac{1}{48 \pi} \frac{m_{\tilde{X}}^{5}}{m_{3 / 2}^{2} M_{*}^{2}}
$$

with $m_{\tilde{X}}$ being the mass of $\tilde{X}$, and it becomes large as the gravitino mass gets small. This decay process produces gravitinos as $\dot{n}_{3 / 2}+3 H n_{3 / 2}=\Gamma(\tilde{X} \rightarrow \psi+X) n_{\tilde{X}}$ where $H$ is the expansion rate of the Universe at the given time. ${ }^{\ddagger}$ Here and below,

\footnotetext{
${ }^{\dagger}$ Here, $\psi$ represents the spin $\frac{1}{2}$ field, though the gravitino has spin $\frac{3}{2}$. In the high energy limit, $\psi$ is related to the longitudinal (helicity $\pm 1 / 2$ ) component of the gravitino, $\psi_{1 / 2}^{\mu}$, as $\psi_{1 / 2}^{\mu} \sim \sqrt{2 / 3} \partial^{\mu} \psi / m_{3 / 2}$.

If the gravitino number density is large, there is also a damping term because of the detailed balance, (r.h.s.) $=\Gamma(\tilde{X} \rightarrow \psi+X) n_{\tilde{X}}\left(1-n_{3 / 2} / n_{3 / 2}^{\text {eq }}\right)$, where $n_{3 / 2}^{\text {eq }}$ is the thermal equilibrium value of $n_{3 / 2}$.
} 
$n_{3 / 2}$ is the number density of gravitinos in the Universe at a given time. If the gravitino mass is in the range $2 h^{2} \mathrm{keV} \lesssim m_{3 / 2} \lesssim 100 \mathrm{keV}$, the decay rate becomes so large that the decay process overproduces the gravitino once the sparticles are thermalized [79]. Thus, if the gravitino mass is in this range, $T_{\max }$ should be lower than about $m_{\tilde{X}} \sim 100 \mathrm{GeV}-1 \mathrm{TeV}$ depending on the mass spectrum of superparticles, or the Universe is overclosed. ${ }^{\S}$

If the gravitino mass is heavier than $O(100 \mathrm{keV})$, the decay process becomes unimportant and the most important production mechanisms of gravitinos are scattering processes. In this case, the Boltzmann equation for the number density of the gravitino, $n_{3 / 2}$, is given by

$$
\dot{n}_{3 / 2}+3 H n_{3 / 2}=\Sigma_{\text {tot }} n_{\text {rad }}^{2}
$$

where $H$ is the expansion rate of the Universe, $\Sigma_{\text {tot }}$ is the thermally averaged total cross section, and $n_{\mathrm{rad}}=\left(\zeta(3) / \pi^{2}\right) T^{3}$. At high energies the first term in Eq. (4.2.2) becomes more significant than the second one, and hence $\Sigma_{\text {tot }}$ is as large as $O\left(g_{3}^{2} m_{\mathrm{G} 3}^{2} / m_{3 / 2}^{2} M_{*}^{2}\right)$. After a detailed calculation, one obtains [79]

$$
\Sigma_{\mathrm{tot}} \sim 5.9 \frac{g_{3}^{2} m_{\mathrm{G} 3}^{2}}{m_{3 / 2}^{2} M_{*}^{2}}
$$

where $g_{3}$ and $m_{\mathrm{G} 3}$ are the gauge coupling constant and the gaugino mass for SU(3) c. Solving Eq. (4.2.4), and taking account of the effect of the dilution factor,

\footnotetext{
$\S^{\S} \mathrm{A}$ similar argument can be applied to the decay process of the particles in the messenger sector or the SUSY breaking sector. In that case, the decay rate becomes much larger since the parent particle is much heavier. Thus, if the particles in those sectors are thermalized, the lower bound on the gravitino mass becomes more stringent than $\sim 100 \mathrm{keV}$.
} 
$g_{*}\left(T_{\max }\right) / g_{*}(T)$ (where $g_{*}(T)$ is the number of the relativistic degrees of freedom in the thermal bath with temperature $T$ ), the number density of the gravitino is given by

$$
\begin{aligned}
\frac{n_{3 / 2}(T)}{n_{\mathrm{rad}}} & =\left.\frac{g_{*}(T)}{g_{*}\left(T_{\max }\right)} \frac{\sum_{\mathrm{tot}} n_{\mathrm{rad}}}{H}\right|_{T=T_{\max }} \\
& \sim 3 \times 10^{-2} \frac{g_{*}(T)}{g_{*}\left(T_{\max }\right)}\left(\frac{m_{3 / 2}}{100 \mathrm{keV}}\right)^{-2}\left(\frac{m_{\mathrm{G} 3}}{1 \mathrm{TeV}}\right)^{2}\left(\frac{T_{\max }}{10 \mathrm{TeV}}\right)
\end{aligned}
$$

Using $g_{*}(T \lesssim 1 \mathrm{MeV}) \sim 3.9$, and $g_{*}\left(T_{\max }\right) \sim 200$, one obtains

$$
\Omega_{3 / 2}=\frac{m_{3 / 2} n_{3 / 2}}{\rho_{\mathrm{c}}} \sim 1 \times h^{-2}\left(\frac{m_{3 / 2}}{100 \mathrm{keV}}\right)^{-1}\left(\frac{m_{\mathrm{G} 3}}{1 \mathrm{TeV}}\right)^{2}\left(\frac{T_{\max }}{10 \mathrm{TeV}}\right)
$$

and the condition $\Omega_{3 / 2} \leq 1$ sets an upper bound on $T_{\max }$. In summary, the upper bound on $T_{\max }$ is given by [79]

$$
T_{\max } \lesssim \begin{cases}100 \mathrm{GeV}-1 \mathrm{TeV} & : 2 h^{2} \mathrm{keV} \lesssim m_{3 / 2} \lesssim 100 \mathrm{keV} \\ 10 \mathrm{TeV} \times h^{2}\left(\frac{m_{3 / 2}}{100 \mathrm{keV}}\right)\left(\frac{m_{\mathrm{G} 3}}{1 \mathrm{TeV}}\right)^{-2}: m_{3 / 2} \gtrsim 100 \mathrm{keV}\end{cases}
$$

The above constraints are summarized in the Fig. 4.1. As one can see, the upper bound on $T_{\max }$ is much lower than the usual reheating temperature after ordinary inflation, $T_{\mathrm{RH}} \gtrsim 10^{8}$. To reduce the number density of the gravitino, therefore, a large entropy production is required.

\subsection{Light Gravitino in the LEGM Models}

The cosmological constraints on a light stable gravitino was discussed in the previous section, and it was shown that one needs to dilute gravitinos produced in the early Universe somehow if $m_{3 / 2} \gtrsim 2 h^{2} \mathrm{keV}$. In this section the gravitino 


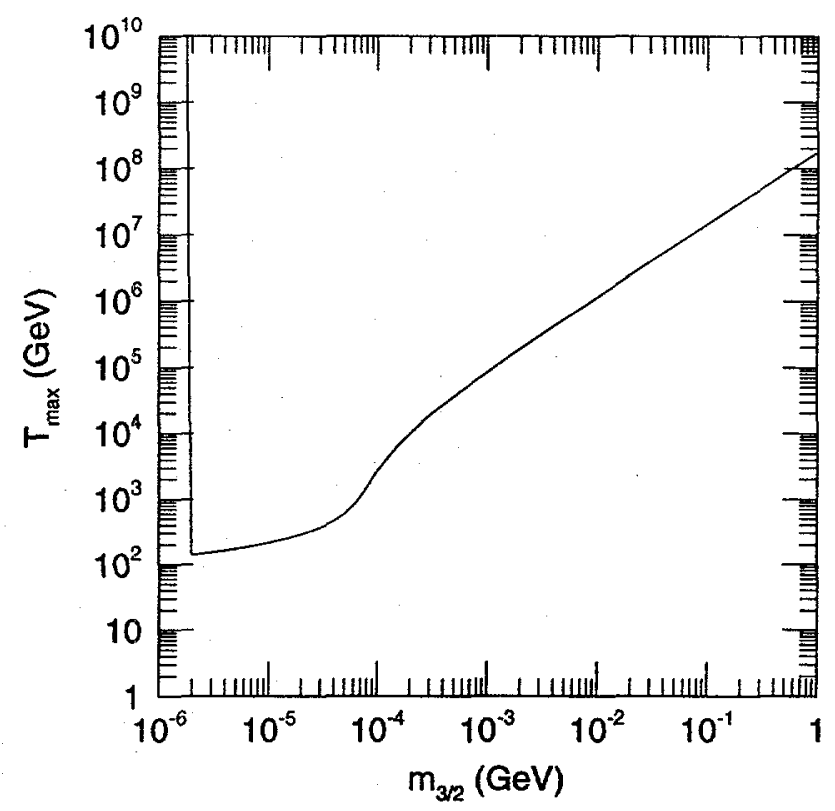

Figure 4.1: The upper bound on $T_{\max }$ as a function of the gravitino mass from the requirement that the relic stable gravitinos do not overclose the Universe. The Hubble parameter is taken to be $H_{0}=100 \mathrm{Mpc} / \mathrm{km} / \mathrm{sec}$. There is no constraint below $m_{3 / 2}=2 \mathrm{keV}$, which is represented by the vertical line. For smaller $H_{0}$, the constraints become more stringent. The upper bound on $T_{\max }$ shifts towards smaller $T_{\max }$ as $\left(H_{0}\right)^{2}$. The vertical line moves towards smaller $m_{3 / 2}$ also as $\left(H_{0}\right)^{2}$. Note that the current data prefer $H_{0} \sim 70 \mathrm{Mpc} / \mathrm{km} / \mathrm{sec}$. 
mass in the LEGM models is estimated carefully and found unlikely to be below $2 h^{2} \mathrm{keV}$.

In the scheme of the LEGM models, there are three sectors: the dynamical SUSY breaking (DSB) sector which originally breaks SUSY, the ordinary sector which consists of the particles in the minimal SUSY standard model (MSSM), and the messenger sector which mediates the SUSY breaking from the DSB sector into the ordinary sector. The scales for these sectors have a large hierarchy, since they are related by loop factors: $\Lambda_{\mathrm{DSB}} \gg \Lambda_{\text {mess }} \gg M_{\mathrm{SUSY}}$, where $\Lambda_{\mathrm{DSB}}, \Lambda_{\text {mess }}$ and $M_{\text {SUSY }}$ represent the scales for the dynamical SUSY breaking sector, messenger sector, and the ordinary sector ( electroweak scale), respectively.

In the LEGM models a gauge interaction, which becomes strong at the scale $\Lambda_{\mathrm{DSB}}$, induces a non-perturbative superpotential. Due to non-perturbative effects, $F$-components of chiral multiplets in the SUSY breaking sector acquire nonvanishing VEVs, $\left\langle F_{0}\right\rangle \sim \Lambda_{\mathrm{DSB}}^{2}$, and SUSY is dynamically broken. Assuming a vanishing cosmological constant, the gravitino mass in this model is given by

$$
m_{3 / 2}=\frac{\left\langle F_{0}\right\rangle}{\sqrt{3} M_{*}} \sim \frac{\Lambda_{\mathrm{DSB}}^{2}}{M_{*}}
$$

In the next stage, the SUSY breaking is fed down to the messenger sector by integrating out the $\mathrm{U}(1)_{\text {mess }}$ interaction. The messenger sector contains a gauge singlet $S$, whose $A$ - and $F$-component $F_{S}$ acquire VEVs after minimizing the potential.* The scale of these VEVS are related to the original SUSY breaking scales $\Lambda_{\mathrm{DSB}}$ as $\langle S\rangle \sim\left\langle F_{S}\right\rangle^{1 / 2} \sim O\left(g_{\mathrm{mess}}^{2} \Lambda_{\mathrm{DSB}} / 16 \pi^{2}\right)$. The ratio of $\left\langle F_{S}\right\rangle$ to $\langle S\rangle$

\footnotetext{
* One actually needs a substantially more complicated messenger sector than the original ones
} 
determines the masses of the sparticles in the ordinary sector. By counting the loop factors, one obtains

$$
\Lambda_{\text {mess }} \equiv \frac{\left\langle F_{S}\right\rangle}{\langle S\rangle}=\kappa_{\text {mess }} \frac{g_{\text {mess }}^{2}}{16 \pi^{2}} \sqrt{m_{3 / 2} M_{*}}
$$

where $\kappa_{\text {mess }}$ is supposed to be of $O(1)$ [74], and $g_{\text {mess }}$ is the gauge coupling constant for the $U(1)_{\text {mess }}$ gauge interaction. In the messenger sector, there are also $5+\overline{5}$ representation of $S U(5)$, i.e. $S U(2)_{L}$ doublets $(L$ and $\bar{L})$ and $S U(3)_{c}$ triplets $(Q$ and $\bar{Q})$. These have the superpotential

$$
W_{\text {mess }}=\lambda_{2} S L \bar{L}+\lambda_{3} S Q \bar{Q} \text {. }
$$

Once $S$ and $F_{S}$ acquire VEVs, the scalar components of $L$ and $\bar{L}(Q$ and $\bar{Q})$ have a mass matrix of the form

$$
\left(\begin{array}{cc}
\lambda^{2}\langle S\rangle^{2} & \lambda\left\langle F_{S}\right\rangle \\
\lambda\left\langle F_{S}\right\rangle & \lambda^{2}\langle S\rangle^{2}
\end{array}\right),
$$

while the fermionic components have mass $\lambda\langle S\rangle$. Therefore, SUSY is broken in the mass spectrum of vector-like $5+\overline{5}$ messenger fields. By integrating out the messenger fields, the soft SUSY breaking parameters are induced in the ordinary sector. With $N_{\mathbf{5}}$ pairs of vector-like $\mathbf{5}+\overline{\mathbf{5}}$ multiplet, the gaugino masses, $m_{\mathrm{G} 1}$, $m_{\mathrm{G} 2}$, and $m_{\mathrm{G} 3}$ are given by

$$
m_{\mathrm{G} i}=\frac{g_{i}^{2}}{16 \pi^{2}} c_{i} \Lambda_{\mathrm{mess}} N_{5}
$$

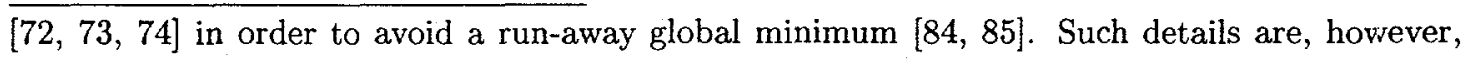
beyond the scope of this chapter. 
where $c_{3}=c_{2}=1$, and $c_{1}=\frac{5}{3}$ in our convention. On the other hand, the masses for the sfermions $m_{\tilde{f}}\left(\tilde{f}=\tilde{u}_{\mathrm{R}}, \tilde{d}_{\mathrm{R}}, \tilde{q}_{\mathrm{L}}, \tilde{l}_{\mathrm{L}}\right.$, and $\left.\tilde{e}_{\mathrm{R}}\right)$ are given by [86]

$$
m_{\tilde{f}}^{2}=2 \Lambda_{\text {mess }}^{2} N_{5} \sum_{i=1}^{3} C_{i}\left(\frac{g_{i}^{2}}{16 \pi^{2}}\right)^{2}
$$

Here, $C_{1}=\frac{5}{3} Y^{2}$ with $Y$ being the usual hypercharge, and $C_{i}=\frac{4}{3}$ and $\frac{3}{4}$ if $\tilde{f}$ is in the fundamental representation of $S U(3)_{c}$ and $S U(2)_{L}$, and $C_{i}=0$ for the gauge singlets.

Combining the above relations with the experimental bounds on the sparticle masses, one can obtain a lower bound on $\Lambda_{\text {mess, }}$, and hence the gravitino mass. A lighter state gives us a more stringent constraint. For most parameters, the lightest among the sfermions is the right handed selectron, whose mass $m_{\tilde{e}_{\mathrm{R}}}$ is given by ${ }^{\dagger}$

$$
m_{\tilde{e}_{\mathrm{R}}}^{2} \sim \frac{10}{3}\left(\frac{g_{1}^{2}\left(\Lambda_{\mathrm{mess}}\right)}{16 \pi^{2}}\right)^{2} \Lambda_{\text {mess }}^{2} N_{5}-m_{Z}^{2} \sin ^{2} \theta_{\mathrm{W}} \cos 2 \beta
$$

For $\tan \beta$ close to 1 , the right-handed selectron mass gives us a stringent bound on the messenger scale. Even if one adopts a conservative constraint of $m_{\tilde{e}_{\mathrm{R}}} \geq$

\footnotetext{
In fact, the selectron mass receives a correction from renormalization effects. However, the correction is less than $10 \%$, and the following arguments are almost unchanged even if one includes such effects.
} 


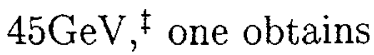

$$
\Lambda_{\text {mess }} \gtrsim 2 \times 10^{4} \mathrm{GeV} \times \frac{1}{\sqrt{N_{5}}}
$$

If $\tan \beta$ is large, the $D$-term contribution enhances the right handed selectron mass, and $m_{\tilde{e}_{\mathrm{R}}}$ can be larger than the experimental limit with smaller value of $\Lambda_{\text {mess. }}$ In that case, however, the sneutrino mass $m_{\tilde{\nu}_{\mathrm{L}}}$

$$
m_{\tilde{\nu}_{\mathrm{L}}}^{2} \sim\left\{\frac{3}{2}\left(\frac{g_{2}^{2}\left(\Lambda_{\text {mess }}\right)}{16 \pi^{2}}\right)^{2}+\frac{5}{6}\left(\frac{g_{1}^{2}\left(\Lambda_{\text {mess }}\right)}{16 \pi^{2}}\right)^{2}\right\} \Lambda_{\text {mess }}^{2} N_{5}+\frac{1}{2} m_{Z}^{2} \cos 2 \beta
$$

receives a negative contribution from the $D$-term, and $\Lambda_{\text {mess }}$ is still constrained to be larger than $\sim 2 \times 10^{4} \mathrm{GeV}$. Therefore, the bound (4.3.8) holds for all values of $\tan \beta$.

In order to translate the above constraint (4.3.8) into a lower bound on the gravitino mass, one needs information about the gauge coupling constant of the $\mathrm{U}(1)_{\text {mess }}$; larger $g_{\text {mess }}$ (at the messenger scale) gives us a less stringent constraint on the gravitino mass. However, if $g_{\text {mess }}$ is too large at the messenger scale, it blows up below the Planck scale or even below the GUT scale. $\S$ By using the 1-loop renormalization group equation, the $\mathrm{U}(1)_{\text {mess }}$ gauge coupling constant at

\footnotetext{
$\ddagger$ LEP-II has placed stronger limits on the $m_{\tilde{e}}$, but as a function of the neutralino mass. The mass difference between $m_{\tilde{e}}$ and the lightest neutralino $\sim \tilde{B}$ is not large in the LEGM models, and the constraint weakens substantially in this situation. The model-independent LEP bound is used because of this reason.

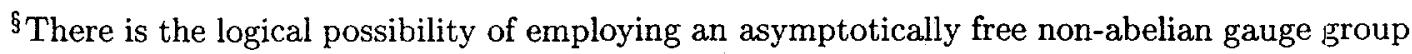
as the messenger group, and assume that its scale parameter is very close to $\Lambda_{\mathrm{DSB}}$. This would, however, require an exponential fine-tuning of parameters.
} 
the messenger scale is constrained by

$$
\frac{g_{\text {mess }}^{2}\left(\Lambda_{\text {mess }}\right)}{16 \pi^{2}} \lesssim \frac{1}{b_{\text {mess }} \ln \left(\Lambda_{\text {cut }}^{2} / \Lambda_{\text {mess }}^{2}\right)}
$$

where $\Lambda_{\text {cut }}$ is the cutoff scale where the perturbative picture may break down, and $b_{\text {mess }}=\sum_{A} Q_{\text {mess }, A}^{2}$ is the sum of the squared charge of the messengers. Typically, $b_{\text {mess }} \sim 10$. (For the model proposed in [73], $b_{\text {mess }}=\frac{34}{3}$, and for a model given in $[74], b_{\text {mess }}=14$.) Assuming $b_{\text {mess }}=10$ and $\Lambda_{\text {cut }} \sim M_{\mathrm{GUT}}, g_{\text {mess }}$ at the messenger scale is constrained to be smaller than $\sim 0.5$, and

$$
m_{3 / 2} \gtrsim 70 \mathrm{keV} \times \frac{\kappa_{\text {mess }}^{-2}}{N_{5}}\left(\frac{g_{\text {mess }}}{0.5}\right)^{-2}\left(\frac{m_{\tilde{e}_{\mathrm{R}}}}{45 \mathrm{GeV}}\right)^{2}
$$

In the minimal model, $N_{\mathbf{5}}=1$, and if the perturbative unification of the gauge coupling constants in the MSSM is assumed, $N_{5} \leq 4$ [75]. Therefore, in any case, the lower bound above is about one or two order of magnitude larger than the cosmological upper bound (4.2.1). Notice that the lower bound on the gravitino mass increases as the experimental lower bound on the sparticle masses increases. 9

Based on the above estimations, the canonical set of the parameters is defined for the following analysis:

$$
m_{3 / 2}=100 \mathrm{keV} \text {, }
$$

IA collorary to this analysis is that it is unlikely to see the decay of a sparticle into the gravitino inside a collider detector. This casts some doubts on the naturalness of $l l \gamma \gamma$ signature at CDF in the LEGM models. A possible way out is to employ the vector-like model [87] and couple a singlet field directly to the messenger fields in the superpotential [88]. This model, however, probably suffers from a tunneling to a color- and charge-breaking supersymmetric minimum if all coupling constants are $O(1)$ [85]. 


$$
\begin{aligned}
& \Lambda_{\text {mess }}=3 \times 10^{4} \mathrm{GeV}, \\
& \left\langle F_{S}\right\rangle^{1 / 2}=\langle S\rangle=\Lambda_{\text {mess }} .
\end{aligned}
$$

Note that it is easy to raise the gravitino mass; one only has to assume a smaller value for the gauge coupling constant for the $\mathrm{U}(1)_{\text {mess. }}$. In the following analysis, the above set of parameters is basically assumed, and a discussion of how the results change as the parameters vary is included.

The above estimations are based on perturbative calculations, and one may worry that a strong coupling in the dynamical sector may allow one to lower the gravitino mass. Such a scenario seems unlikely, however. To see this, it is convenient to define the "vacuum polarizations" from the DSB sector for the $\mathrm{U}(1)_{\text {mess }}$ gauge multiplet:

$$
\begin{aligned}
& \text { F.T. }\left\langle 0\left|T\left(A_{\mu} A_{\nu}\right)\right| 0\right\rangle_{1 \mathrm{PI}}=i q^{2} \Pi_{A}\left(q^{2}\right) g_{\mu \nu}, \\
& \text { F.T. }\langle 0|T(\lambda \lambda)| 0\rangle_{1 \mathrm{PI}}=(-i)\left\{g \Pi_{\lambda}\left(q^{2}\right)+\Sigma\left(q^{2}\right)\right\}, \\
& \text { F.T. }\langle 0|T(D D)| 0\rangle_{1 \mathrm{PI}}=i \Pi_{D}\left(q^{2}\right),
\end{aligned}
$$

where F.T. stands for the four-dimensional Fourier Transform to the momentum space, and 1PI for one-particle irreducible diagrams. At tree level, $\Pi_{A}=\Pi_{\lambda}=$ $\Pi_{D}=\Sigma=0$. These quantities receive radiative corrections of $O\left(g_{\text {mess }}^{2} / 16 \pi^{2}\right)$ if the perturbative calculation is reliable. The messenger scale in the LEGM model is induced by integrating out the SUSY breaking sector and the U(1) mess gauge multiplet. By using $\Pi_{A}, \Pi_{\lambda}$ and $\Pi_{D}$, the SUSY breaking scalar mass in the 
messenger sector ( $\sim$ the messenger scale) is given by

$$
m_{\mathrm{mess}}^{2} \sim g_{\mathrm{mess}}^{2} \int \frac{d^{4} q}{(2 \pi)^{4} i} \frac{1}{q^{2}}\left\{3 \Pi_{A}\left(q^{2}\right)-4 \Pi_{\lambda}\left(q^{2}\right)+\Pi_{D}\left(q^{2}\right)\right\}
$$

If one is limited to the physics of the DSB sector, there is no pole in the $\Pi$ functions at $q^{2}=0$ which, if present, implies the Higgs mechanism for the $\mathrm{U}(1)_{\text {mess }}$ gauge group. The only singularities in $\Pi$ functions are, therefore, branch cuts which appear above certain threshold $q^{2} \gtrsim \Lambda_{\mathrm{DSB}}^{2}$ which is the only scale in the problem. Then the integrations in Eq. (4.3.18) can be Wick rotated and one obtains

$$
m_{\mathrm{mess}}^{2} \sim-\frac{g_{\mathrm{mess}}^{2}}{16 \pi^{2}} \int_{0}^{\infty} d q_{E}^{2}\left\{3 \Pi_{A}\left(-q_{E}^{2}\right)-4 \Pi_{\lambda}\left(-q_{E}^{2}\right)+\Pi_{D}\left(-q_{E}^{2}\right)\right\}
$$

Now it is clear that the integration is purely Euclidean, and hence all $\Pi$ functions are far off-shell. Thus, the perturbative result is essentially reliable even when the DSB sector is strongly coupled. It is also useful to recall that similar calculations of vacuum polarization amplitudes in QCD tend to agree with lowest order perturbative results for the running of the fine-structure constant, or the scaled-up QCD estimate of the electroweak $S$-parameter." It is therefore concluded that there is no significant enhancement of the resulting $m_{\text {mess }}^{2}$, and hence the estimates of the

\footnotetext{
"Note that the $S$-parameter is defined by the vacuum polarization amplitudes at $q^{2}=0$, and hence more sensitive to the non-perturbative effects than Eq. (4.3.19), which smears them over a wide range of $q^{2}$. Still, a perturbative estimate of $S$ differs from the scaled-up QCD only by a factor of two. One may also estimate the $S$-parameter by assuming that it is dominated by the $\rho$ and $a_{1}$ poles. Then the result is obtained by the tree-level process. Even so, the coupling of the resonances to the current operator has a factor of $1 / 4 \pi$ and the counting of $1 / 4 \pi$ factors remains the same in as the perturbative one-loop result.
} 
messenger scale and the resulting gravitino mass (4.3.11) can be trusted.

The constraint (4.3.11) sets severe bounds on cosmology. In particular, some mechanism to generate a dilution factor of $\sim\left(m_{3 / 2} / 2 h^{2} \mathrm{keV}\right)$ at a relatively low temperature below the upper bound on the maximum temperature given in Eq. (4.2.8) and in Fig. 4.1 is needed, if the gravitino mass is larger than $2 h^{2} \mathrm{keV}$. Furthermore, even if one adopts such a large entropy production at a low temperature, baryogenesis may still be a problem. The Affleck-Dine mechanism [80] for baryogenesis is one of the possibilities to generate baryons at a relatively low temperature.**

However, in the LEGM models, the behavior of the flat direction at large amplitude is quite different from the usual supergravity case. Thus, even if one assumes the Affleck-Dine mechanism, it is a non-trivial question whether there can be enough baryon number density. In the following sections, this possibility is pursued, and as a result, it will be shown that the Affleck-Dine mechanism works sufficiently well, enough to explain the present value of the baryon-to-entropy ratio.

**Electroweak baryogenesis may be another possibility to generate baryon asymmetry at relatively low temperatures. However, the resulting baryon-to-photon ratio depends on the details of the complicated dynamics of the phase transition. Furthermore, the generated baryon asymmetry would not be large, if any, and would probably not survive a huge entropy production to dilute the string moduli fields as discussed in Sec. 4.6. 


\subsection{Flat Directions in the LEGM Models}

As discussed in the previous sections, the constraint from the gravitino cosmology is quite severe in models with the LEGM. Therefore, it is preferable to look for baryogenesis scenarios which do not require high temperatures.

The discussion will focus on the Affleck-Dine baryogenesis in the LEGM models in this chapter. There is one crucial difference from hidden sector models: the potential along the MSSM flat directions is not simply parabolic. Therefore the form of the potential is discussed first in this section, and its implication to the Affleck-Dine baryogenesis are discussed in the next section.

In hidden sector models, where the SUSY breaking effect is mediated by Planck-scale operators, the soft SUSY breaking parameters are actually "hard", in the sense that they renormalize as usual mass terms between the Planck-scale and the weak scale. On the other hand, the SUSY breaking scalar mass terms are suppressed beyond the messenger scale in the LEGM models.

This is analogous to the situation in the QCD. The current masses of the quarks renormalize according to the ordinary perturbation theory. They are "hard" masses. However the constituent quark masses are suppressed by a power of the energy: "soft". This is because the constituent quark masses are dynamically generated by the spontaneous chiral symmetry breaking, which is characterized by the order parameter $\langle\bar{q} q\rangle$. The constituent quark mass has to be proportional to this order parameter. At high momentum transfer, a dimensional analysis then 
indicates that the effective constituent mass behaves as $m_{\text {const }}\left(Q^{2}\right) \sim\langle\bar{q} q\rangle / Q^{2}$.

The same argument applies to the soft SUSY breaking masses from the LEGM. SUSY is broken by an $F$-component of a chiral superfield, $\left\langle F_{S}\right\rangle \neq 0$. The soft SUSY breaking scalar mass is necessarily proportional to the order parameter of SUSY breaking, i.e., $m^{2} \propto\left\langle F_{S}\right\rangle^{\dagger}\left\langle F_{S}\right\rangle$. A dimensional analysis indicates that it is suppressed at high momentum transfers, $m^{2}\left(Q^{2}\right) \sim\left\langle F_{S}\right\rangle^{\dagger}\left\langle F_{S}\right\rangle / Q^{2}$. Therefore, the SUSY breaking parameters "shut off" at high energies.

The potential of a MSSM flat direction is given simply by $V=m^{2}|\phi|^{2}$, where $m^{2}$ is a soft SUSY breaking mass. A renormalization group improvement yields $V=m^{2}\left(|\phi|^{2}\right)|\phi|^{2}$. In the hidden sector case, $m^{2}\left(|\phi|^{2}\right)$ has only a logarithmic dependence on $|\phi|^{2}$ and hence can be taken approximately constant unless it crosses zero at some energy scale. For most cosmological applications, this is a sufficiently good description. In the LEGM models, however, the effective mass $m^{2}\left(|\phi|^{2}\right)$ exhibits a power dependence on $|\phi|^{2}$ which cannot be neglected. One expects that $m^{2}\left(|\phi|^{2}\right)$ behaves as $\left\langle F_{S}\right\rangle^{\dagger}\left\langle F_{S}\right\rangle /|\phi|^{2}$ for large $|\phi|$, and hence the potential behaves approximately like a constant for $|\phi|>\langle S\rangle$, which is the mass scale of the messengers.

An explicit two-loop calculation of the effective potential $V(\phi)$ was performed, and its details are presented in Appendix A. Here only the result is quoted. As expected, the potential behaves parabolically around the origin, while it becomes approximately constant for large $|\phi|$; actually it keeps growing slowly as $\left(\ln |\phi|^{2}\right)^{2}$. 
The potential of a MSSM flat direction behaves as

$$
V(\phi) \sim\left(\frac{g^{2}}{(4 \pi)^{2}}\right)^{2}\left(\frac{\left\langle F_{S}\right\rangle}{\langle S\rangle}\right)^{2}|\phi|^{2}+O\left(|\phi|^{4}\right)
$$

for small $|\phi| \ll\langle S\rangle$, and

$$
V(\phi) \sim V_{0}\left(\ln \frac{|\phi|^{2}}{\langle S\rangle^{2}}\right)^{2}
$$

with

$$
V_{0} \sim \frac{g^{2}}{(4 \pi)^{4}}\left\langle F_{S}\right\rangle^{2} \sim\left(3 \times 10^{3} \mathrm{GeV}\right)^{4}
$$

for large $|\phi| \gg\langle S\rangle$. Here, $g$ generically refers to standard model gauge coupling constants.*

For extremely large $|\phi|$, however, the contribution from supergravity becomes important. Supergravity generates a contribution to the scalar potentials $\sim m_{3 / 2}^{2}|\phi|^{2}$ for any $|\phi|{ }^{\dagger}$ To determine the relative importance of the LEGM and supergravity contributions, one should compare their derivatives $V^{\prime}$, because this is the quantity which appears in the equation of motion. The derivative of the

*One may wonder why Eqs. (4.4.2) and (4.4.3) have only two powers of gauge coupling constants despite the two-loop-ness of the effective potential. This is the result of an explicit calculation, and it can also be explained in a simple way. When the field value is large, the standard model gauge multiplets acquire large masses of order $g \phi$. The effective potential is generated by the exchange of heavy gauge multiplets, and hence it is suppressed by $1 /|g \phi|^{2}$. This cancels two powers in gauge coupling constants.

${ }^{\dagger}$ This is true for the minimal supergravity and its variants. This contribution, however, is not there in no-scale supergravity, or in general, in supergravity with Heisenberg symmetry [89, 90]. In such a case, $\phi_{\mathrm{eq}}$ must be taken at $M_{*}$ in the rest of the discussions. 
potential from the LEGM is

$$
\frac{\partial V}{\partial \phi} \sim V_{0} \frac{2 \phi^{*}}{|\phi|^{2}}\left(\ln \frac{|\phi|^{2}}{\langle S\rangle^{2}}\right)
$$

which is to be compared with the supergravity contribution $\partial V / \partial \phi=m_{3 / 2}^{2} \phi^{*}$. The supergravity contribution is more important above a threshold value $\phi_{\text {eq }}$ which is given by

$$
\begin{aligned}
\phi_{\mathrm{eq}} & \sim\left\{\frac{2 V_{0}}{m_{3 / 2}^{2}}\left(\ln \frac{\left|\phi_{\mathrm{eq}}\right|^{2}}{\langle S\rangle^{2}}\right)\right\}^{1 / 2} \\
& \sim 7 \times 10^{11} \mathrm{GeV} \times\left(\frac{m_{3 / 2}}{100 \mathrm{keV}}\right)^{-1}\left(\frac{V_{0}^{1 / 4}}{3 \times 10^{3} \mathrm{GeV}}\right)^{2}
\end{aligned}
$$

The motion of the flat direction is determined by the effective potential given in this section and the canonical kinetic term, and there is no need to include the wave function renormalization factor at this order in perturbation theory. See Appendix B for details.

\subsection{Affleck-Dine Baryogenesis in the LEGM Models}

The goal of this section is to estimate the size of the baryon-to-entropy ratio from Affleck-Dine baryogenesis in the LEGM models. Because of the multiple scales in the problem, the discussion becomes somewhat complicated. The basic conclusion is that the Affleck-Dine baryogenesis works as efficiently as in the hidden sector case, but in a much more non-trivial manner. Finally the possible dilution of gravitinos via the decay of the Affleck-Dine flat direction is discussed, and it is determined that the gravitinos can be diluted below the closure limit if 
the initial amplitude of the flat direction is sufficiently large.

\subsubsection{Generalities}

In Affleck-Dine baryogenesis [80], one assumes that a MSSM flat direction has a large amplitude at the end of the primordial inflation. The mechanism to achieve a large amplitude varies: a negative curvature from a non-minimal Kähler potential [91], or no-scale supergravity [90]. In any case, it tends to be equal to or larger than the expansion rate of the Universe during inflation $H_{\text {inf }} \sim 10^{11} \mathrm{GeV}$ - $10^{13} \mathrm{GeV}$ depending on inflationary scenarios. It will be phenomenologically parameterized just as the initial amplitude $\phi_{0}$.

A typical assumption is that there is a baryon-number violating Kähler potential term $K \sim l^{*} q^{*} u^{c} d^{c} / M_{*}^{2}$, where $M_{*}$ is the reduced Planck scale. ${ }^{*}$ The SUSY breaking effects from the LEGM generates a term in the potential ${ }^{\dagger}$

$$
\mathcal{O} \sim \int d^{4} \theta \frac{g^{4}}{(4 \pi)^{4}} \frac{\left|\theta^{2} F_{S}\right|^{2}}{g^{2}|\phi|^{2}}\left(\ln \frac{|\phi|^{2}}{\langle S\rangle^{2}}\right)^{2} \frac{1}{M_{*}^{2}} l^{*} q^{*} u^{c} d^{c}+h . c .
$$

${ }^{*}$ It could also well be the GUT-scale $M_{\mathrm{GUT}}$. However, in this chapter, this form of the baryon number violating operator is assumed for simplicity. The extension to the case with $K \sim l^{*} q^{*} u^{c} d^{c} / M_{\mathrm{GUT}}^{2}$ is trivial, and one can easily estimate the resulting baryon-to-entropy ratio.

${ }^{\dagger}$ This has not been calculated explicitly. This form is expected based on the analogy to the calculation of the effective potential in the previous section. The only difference is that the previous one arises from the kinetic term $\phi^{*} \phi$ in the Kähler potential rather than from a non-renormalizable term $l^{*} q^{*} u^{c} d^{c} / M_{*}^{2}$ here. 


$$
=\frac{V_{0}}{|\phi|^{2}}\left(\ln \frac{|\phi|^{2}}{\langle S\rangle^{2}}\right)^{2} \frac{1}{M_{*}^{2}} \tilde{l}^{*} \tilde{q}^{*} \tilde{u}^{c} \tilde{d}^{c}+h . c .
$$

while the supergravity effect induces an operator in the scalar potential

$$
\mathcal{O} \sim \int d^{4} \theta \frac{\left|\theta^{2} F_{0}\right|^{2}}{M_{*}^{2}} \frac{1}{M_{*}^{2}} l^{*} q^{*} u^{c} d^{c}+h . c .=\frac{m_{3 / 2}^{2}}{M_{*}^{2}} \tilde{l}^{*} \tilde{q}^{*} \tilde{u}^{c} \tilde{d}^{c}+h . c .
$$

The LEGM operator is dominant if $\left|\phi_{0}\right| \lesssim \phi_{\text {eq }}$, while the supergravity one dominates if $\left|\phi_{0}\right| \gtrsim \phi_{\text {eq }}$. Therefore, the two cases are discussed separately below. In either case, the size of the baryon-number violating operator is much smaller than in the hidden sector case (Appendix E). It turns out, however, that the baryogenesis proceeds efficiently with these operators.

Below, the fields are generically referred to as $\phi$, without distinction amnong various species. The baryon number in the scalar sector is given by

$$
n_{B}=i\left(\dot{\phi}^{*} \phi-\dot{\phi} \phi^{*}\right)
$$

while the baryon number violating operator is written as

$$
\mathcal{O} \sim\left[m_{3 / 2}^{2}+\frac{V_{0}}{|\phi|^{2}}\left(\ln \frac{|\phi|^{2}}{\langle S\rangle^{2}}\right)^{2}\right] \frac{1}{M_{*}^{2}}\left(\phi^{4}+\phi^{* 4}\right)
$$

\subsection{2 $\left|\phi_{0}\right| \gtrsim \phi_{\text {eq }}$}

For sufficiently large $\left|\phi_{0}\right|\left(\left|\phi_{0}\right| \gtrsim \phi_{\mathrm{eq}}\right)$, the supergravity contribution is initially important, and the field begins to roll down the potential when the expansion rate of the Universe $H$ is comparable to $H \sim m_{3 / 2}$. First, the primordial baryon number asymmetry will be estimated for $\left|\phi_{0}\right| \gtrsim \phi_{\mathrm{eq}}$. 
A rough estimation of the baryon asymmetry, which is generated just after the start of the oscillation of the $\phi$ field can be done only by using simple order of magnitude arguments. With the above baryon number violating operator, the time evolution of the baryon number is given by

$$
\dot{n}_{B}+3 H n_{B}=i\left(\frac{\partial \mathcal{O}}{\partial \phi} \phi-\frac{\partial \mathcal{O}}{\partial \phi^{*}} \phi^{*}\right)
$$

When the field begins to roll down the potential, its initial motion is slow, and one can neglect the $\dot{n}_{B}$ term in the equation (see Appendix D). Then the resulting baryon number can be estimated by [92]

$$
n_{B} \sim \frac{i}{3 H}\left(\frac{\partial \mathcal{O}}{\partial \phi} \phi-\frac{\partial \mathcal{O}}{\partial \phi^{*}} \phi^{*}\right)
$$

Using the approximate order of magnitude of the operator and $H \sim m_{3 / 2}$, one obtains

$$
n_{B} \sim \frac{m_{3 / 2} \operatorname{Im}\left(\phi_{0}^{4}\right)}{M_{*}^{2}}
$$

It depends on the imaginary part of the initial amplitude. The entropy of the radiation at this stage is given by $s \sim g_{*} T^{3}$ while the energy density $\rho_{\text {rad }} \sim g_{*} T^{4} \sim$ $m_{3 / 2}^{2} M_{*}^{2}$. By putting them together, one can estimate the baryon-to-entropy ratio,

$$
\frac{n_{B}}{s} \sim g_{*}^{-1 / 4} \frac{\operatorname{Im}\left(\phi_{0}^{4}\right)}{m_{3 / 2}^{1 / 2} M_{*}^{7 / 2}} \sim 4 \times 10^{10} \times\left(\frac{\left|\phi_{0}\right|}{M_{*}}\right)^{4}\left(\frac{m_{3 / 2}}{100 \mathrm{keV}}\right)^{-1 / 2} \sin 4 \theta_{0},
$$

where the initial amplitude is parameterized as $\phi_{0}=\left|\phi_{0}\right| e^{i \theta_{0}}$. As one can see, a large baryon asymmetry can be generated, if the initial amplitude of $\phi$ is not too small. Therefore, a large enough baryon number can remain in this scenario, even if there is a substantial entropy production after the Affleck-Dine baryogenesis. 
Note that one obtains exactly the same expression in the hidden sector models, but with a different $m_{3 / 2}$.

The present baryon-to-entropy ratio is also given by the above formula, if there is no significant entropy production. However, in a realistic situation, there can be entropy production. In particular, the decay of the Affleck-Dine field $\phi$ may produce a large amount of entropy. Furthermore, for $m_{3 / 2} \gtrsim 2 h^{2} \mathrm{keV}$, a non-negligible entropy production is needed to dilute the primordial gravitino. If there is an entropy production after the Affleck-Dine baryogenesis, the primordial baryon number density is also diluted. In the following, how an entropy production affects the results is discussed.

The entropy production due to the decay of the flat direction can be estimated. As discussed in the previous section, $\phi$ starts to oscillate when $T=T_{0} \sim$ $g_{*}^{-1 / 4} \sqrt{m_{3 / 2} M_{*}}$, if $\left|\phi_{0}\right| \gtrsim \phi_{\mathrm{eq}}$. During $|\phi| \gtrsim \phi_{\mathrm{eq}}$, the potential for $\phi$ is dominated by the supergravity contribution, and hence

$$
|\phi|^{2} R^{3}=\text { const. } \quad\left(\phi_{\text {eq }} \lesssim|\phi| \lesssim\left|\phi_{0}\right|\right)
$$

Thus, the temperature at $|\phi| \sim \phi_{\text {eq }}$, which is denoted $T_{\text {eq }}$, is estimated as

$$
T_{\mathrm{eq}} \sim T_{0}\left(\frac{\phi_{\mathrm{eq}}}{\left|\phi_{0}\right|}\right)^{2 / 3}
$$

For $|\phi| \lesssim \phi_{\text {eq }}$, the potential for the flat direction is dominated by the LEGM piece, and the evolution for $\phi$ does not obey the relation (4.5.9). By using the virial theorem, the evolution of the flat direction can be estimated, and is given 
by

$$
|\phi| R^{3}=\text { const. } \quad\left(\langle S\rangle \lesssim|\phi| \lesssim \phi_{\text {eq }}\right)
$$

(See Appendix C.) By using the above relations, one obtains the dilution factor due to the decay of the flat direction.

Now an important question is at what field amplitude $\phi$ decays into radiation. When the motion is dominated by a parabolic term, the time dependence of the oscillation is known (just a harmonic oscillator), and one can calculate the rate of particle production in such a background. The result is known to be the same as the single particle decay rate, if the amplitude is not too large compared to the oscillation frequency. Once the amplitude becomes comparable to $\langle S\rangle$, the potential is almost parabolic, and one finds that the coherent oscillation decays into radiation rapidly. On the other hand, a corresponding calculation is difficult when the potential is dominated by the logarithmic term. In the analysis, the decay amplitude, $\phi_{\mathrm{dec}}$ is regarded, as a free parameter, and the $\phi_{\mathrm{dec}}$-dependence of the results is discussed. ${ }^{\ddagger}$

The temperature of the background radiation at the decay time of the flat

\footnotetext{
${ }^{\mathfrak{t} F o r}$ a canonical parameter, $\phi_{\mathrm{dec}} \sim 10^{5} \mathrm{GeV}$ is considered for estimating dilution factors. Since $\phi$ decays at $\phi \sim\langle S\rangle \sim 3 \times 10^{4} \mathrm{GeV}$ at latest, this choice gives the minimum estimate of the baryon asymmetry. If $\phi$ decays earlier, the dilution factor is less and the baryon asymmetry is larger. The dilution factor is likely to be overestimated with this choice. This point will be addressed later, during the discussion of a possible dilution of gravitinos from the decay of the flat direction.
} 
direction, $T_{\mathrm{dec}}$, is given by

$$
T_{\mathrm{dec}} \sim T_{\mathrm{eq}}\left(\frac{\phi_{\mathrm{dec}}}{\phi_{\mathrm{eq}}}\right)^{1 / 3} \sim T_{0}\left(\frac{\phi_{\mathrm{dec}}}{\phi_{\mathrm{eq}}}\right)^{1 / 3}\left(\frac{\phi_{\mathrm{eq}}}{\left|\phi_{0}\right|}\right)^{2 / 3}
$$

On the contrary, the energy density of the flat direction is

$$
\rho_{\text {flat }} \sim V_{0}
$$

Then, if $\rho_{\text {flat }} \gtrsim \rho_{\text {rad }}$, the dilution factor from the decay is given by

$$
\begin{aligned}
D & \sim\left(\frac{\rho_{\text {flat }}}{\rho_{\text {rad }}}\right)^{3 / 4} \sim\left(\frac{V_{0}}{g_{*} T_{\text {dec }}^{4}}\right)^{3 / 4} \\
& \sim 6 \times 10^{8}\left(\frac{\left|\phi_{0}\right|}{M_{*}}\right)^{2}\left(\frac{\phi_{\text {dec }}}{10^{5} \mathrm{GeV}}\right)^{-1}\left(\frac{m_{3 / 2}}{100 \mathrm{keV}}\right)^{-1 / 2}\left(\frac{V_{0}^{1 / 4}}{3 \times 10^{3} \mathrm{GeV}}\right)
\end{aligned}
$$

Note that $D \sim 1$ if $\rho_{\text {flat }} \lesssim \rho_{\text {rad }}$, or in terms of the initial amplitude, $\phi_{0} \lesssim 10^{14} \mathrm{GeV}$.

Combining the above dilution factor with the estimation of the primordial baryon number density given in Eq. (4.5.8), one obtains the present baryon number asymmetry. In order to make a pessimistic estimate of the resulting baryon asymmetry, it is assumed that the flat direction decays only when its amplitude is as small as $\langle S\rangle$. This assumption maximizes the entropy production, and hence, gives the minimum value for the baryon asymmetry. If it decays earlier, then the entropy production is less and hence the baryon asymmetry is larger. With this caveat in mind, one can make an estimate of the resulting baryon-to-entropy ratio, and in the case with entropy production $(D>1)$, the resulting baryon-to-entropy ratio is given by

$$
\begin{aligned}
\frac{n_{B}}{s} & \sim D^{-1} g_{*}^{-1 / 4} \frac{\operatorname{Im}\left(\phi_{0}^{4}\right)}{m_{3 / 2}^{1 / 2} M_{*}^{7 / 2}} \\
& \sim 70 \times\left(\frac{\left|\phi_{0}\right|}{M_{*}}\right)^{2}\left(\frac{\phi_{\mathrm{dec}}}{10^{5} \mathrm{GeV}}\right)\left(\frac{V_{0}^{1 / 4}}{3 \times 10^{3} \mathrm{GeV}}\right) \sin 4 \theta_{0}
\end{aligned}
$$


Note that the result is independent of the gravitino mass. It is intriguing that the final result is more or less the same as in the hidden sector case Eq. (E.9).

\subsection{3 $\left|\phi_{0}\right| \lesssim \phi_{\text {eq }}$}

Next, the case of $\left|\phi_{0}\right| \lesssim \phi_{\text {eq }}$ is discussed. ${ }^{\S}$ In this case, the potential for the flat direction is dominated by the LEGM-piece, and the flat direction starts to move when $H \sim \sqrt{\left|V^{\prime}\left(\phi_{0}\right)\right| /\left|\phi_{0}\right|} \sim \sqrt{V_{0}\left(\ln \left|\phi_{0}\right|^{2} /\langle S\rangle^{2}\right)} /\left|\phi_{0}\right|$. The temperature at this stage, $T_{0}$, is estimated as

$$
T_{0}^{2} \sim g_{*}^{-1 / 2} M_{*} \frac{V_{0}^{1 / 2}}{\left|\phi_{0}\right|}\left(\ln \frac{\left|\phi_{0}\right|^{2}}{\langle S\rangle^{2}}\right)^{1 / 2}
$$

Then, by using Eq.(4.5.6), one can estimate the resulting baryon number density, and hence the baryon-to-entropy ratio. Note that the baryon-number-violating operator $\mathcal{O}$ is different from the previous case.

Following exactly the same steps as in the previous case, one finds

$$
n_{B} \sim \frac{V_{0}^{1 / 2} \operatorname{Im}\left(\phi_{0}^{4}\right)}{M_{*}^{2}\left|\phi_{0}\right|}\left(\ln \frac{\left|\phi_{0}\right|^{2}}{\langle S\rangle^{2}}\right)^{3 / 2}=\frac{V_{0}^{1 / 2}\left|\phi_{0}\right|^{3}}{M_{*}^{2}}\left(\ln \frac{\left|\phi_{0}\right|^{2}}{\langle S\rangle^{2}}\right)^{3 / 2} \sin 4 \theta_{0}
$$

when the flat direction starts to move. Therefore, the baryon-to-entropy ratio is

$\S$ As noted before, the no-scale supergravity does not generate a potential term proportional to $m_{3 / 2}^{2}$ and hence the evolution of the flat direction is always dominated by the LEGM piece. Then the formulae presented in this subsection must be used even for a larger $\left|\phi_{0}\right| \sim M_{*}$. Such a large $\left|\phi_{0}\right|$ is indeed expected in the no-scale case because the flat directions remain flat even during the inflation [90]. 
given by

$$
\begin{aligned}
\frac{n_{B}}{s} & \sim g_{*}^{-1 / 4} \frac{\left|\phi_{0}\right|^{9 / 2}}{V_{0}^{1 / 4} M_{*}^{7 / 2}}\left(\ln \frac{\left|\phi_{0}\right|^{2}}{\langle S\rangle^{2}}\right)^{3 / 4} \sin 4 \theta_{0} \\
& \sim 6 \times 10^{-14} \times\left(\frac{\left|\phi_{0}\right|}{10^{12} \mathrm{GeV}}\right)^{9 / 2}\left(\frac{V_{0}^{1 / 4}}{3 \times 10^{3} \mathrm{GeV}}\right)^{-1} \sin 4 \theta_{0}
\end{aligned}
$$

which is typically too small. It is useful to note that the above formula is larger by a factor of the logarithm than the corresponding formula Eq. (4.5.8) for the case $\phi_{0} \gtrsim \phi_{\mathrm{eq}}$ when $\phi_{0}=\phi_{\mathrm{eq}}$ is substituted. Of course such a discontinuity cannot exist. It simply means that there is a transition region at $\phi_{0} \sim \phi_{\mathrm{eq}}$ where there is a slight rise in $n_{B} / s$ when $\phi_{0} \sim \phi_{\text {eq }}$ is crossed from above.

The dilution factor can be estimated also along the lines of the previous case. One has

$$
T_{\text {dec }} \sim T_{0}\left(\frac{\phi_{\text {dec }}}{\phi_{0}}\right)^{1 / 3}
$$

and hence (if $\rho_{\text {flat }}>\rho_{\text {rad }}$ ),

$$
\begin{aligned}
D & \sim\left(\frac{\rho_{\text {flat }}}{\rho_{\text {rad }}}\right)^{3 / 4} \sim\left(\frac{V_{0}}{g_{*} T_{\mathrm{dec}}^{4}}\right)^{3 / 4} \sim \frac{\left|\phi_{0}\right|^{5 / 2}}{M_{*}^{3 / 2} \phi_{\mathrm{dec}}\left(\ln \left|\phi_{0}\right|^{2} /\langle S\rangle^{2}\right)^{3 / 4}} \\
& \sim 2 \times 10^{-4}\left(\frac{\left|\phi_{0}\right|}{10^{12} \mathrm{GeV}}\right)^{5 / 2}\left(\frac{\phi_{\mathrm{dec}}}{10^{5} \mathrm{GeV}}\right)^{-1}
\end{aligned}
$$

(More correctly, the dilution factor is $D=\left(\left(\rho_{\text {fat }}+\rho_{\text {rad }}\right) / \rho_{\text {rad }}\right)^{3 / 4}$ and cannot be less than unity.) Therefore, the dilution factor is much less important than in the previous case.

\subsubsection{Numerical Analysis}

A more detailed behavior of the baryon-to-entropy ratio can be studied by a 
numerical calculation. Here, in order to better see the behavior of the results, the resulting baryon-to-entropy ratio for a particular set of parameters: $V_{0}=$ $\left(3 \times 10^{3} \mathrm{GeV}\right)^{4},\langle S\rangle=3 \times 10^{4} \mathrm{GeV}, \phi_{\text {dec }}=10^{5} \mathrm{GeV}$, and $\sin 4 \theta_{0}=1$ is shown. The results for other sets of parameters can be easily estimated by using Eqs.(4.5.8) and (4.5.15). First, the equation of motion for the flat direction,

$$
\ddot{\phi}+3 H \dot{\phi}+\frac{\partial V}{\partial \phi^{*}}=0
$$

is solved, with the potential ${ }^{9}$

$$
V=m_{3 / 2}^{2}|\phi|^{2}+V_{0}\left(\ln \frac{|\phi|^{2}}{\langle S\rangle^{2}}\right)^{2}-\left[m_{3 / 2}^{2}+\frac{V_{0}}{|\phi|^{2}}\left(\ln \frac{|\phi|^{2}}{\langle S\rangle^{2}}\right)^{2}\right] \frac{1}{M_{*}^{2}}\left(\phi^{4}+\phi^{* 4}\right) .
$$

The calculation starts at a temperature much higher than $T_{0}\left(T=10 T_{0}\right)$, and follow the evolution of the flat direction as well as the temperature of the thermal bath. With the initial value $\phi_{0}=0.2 M_{*} e^{i \pi / 8}$, the initial motion is shown in Fig. 4.2. As one can see, $\phi$ starts an elliptical motion due to the baryon-number violating term in the potential. This means that a non-vanishing baryon number is generated once $\phi$ starts to oscillate. The generated baryon number was indeed found to be consistent with the estimates in the previous subsections within a factor of a few.

With the motion of the flat direction, one can calculate the baryon number by using Eq.(4.5.3), and hence the baryon-to-entropy ratio. After several cycles

\footnotetext{
The potential given in Eq.(4.5.22) is unbounded-below for $\phi \gtrsim M_{*}$, and higher-dimension operators are supposed to stabilize it. However, it is only necessary to consider initial amplitudes less than $\sim M_{*}$ in the present analysis, and hence the postulated potential is a good enough approximation.
} 


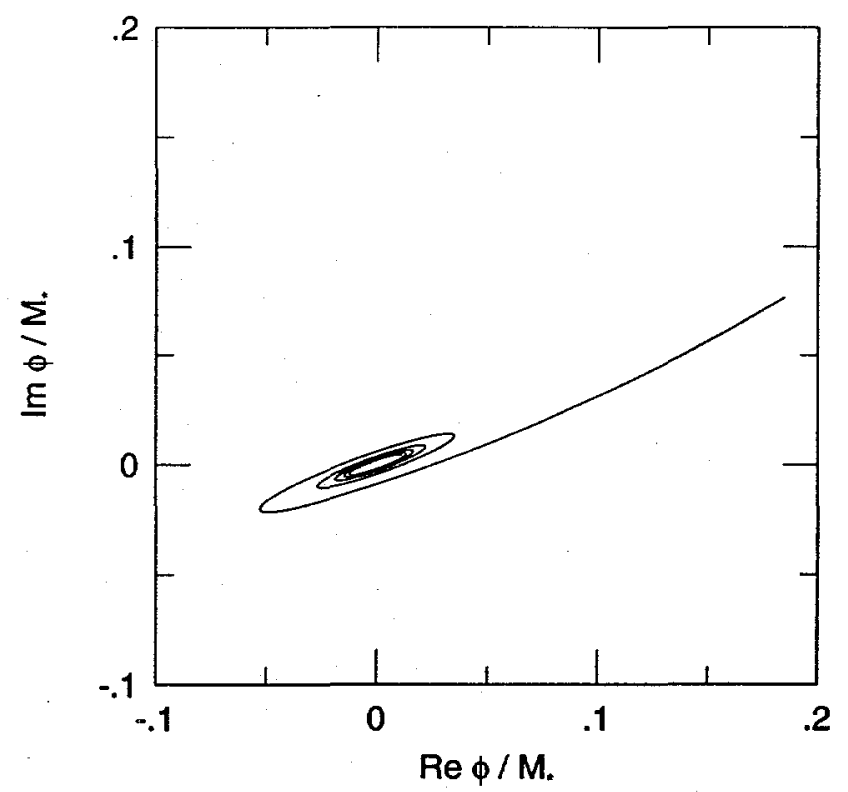

Figure 4.2: The initial motion of the flat direction with the potential given in Eq. (4.5.22). Here, $m_{3 / 2}=100 \mathrm{keV}$, and $\phi_{0}=0.2 M_{*} e^{i \pi / 8}$ was taken. 
of oscillation, the baryon-to-entropy ratio becomes almost constant. Then, the evolution of $\phi$ and $T$ can be easily traced by using Eqs.(4.5.9) and (4.5.11) with $R T=$ const. Finally, the dilution factor, $D=\left(\left(\rho_{\mathrm{rad}}+\rho_{\phi}\right) / \rho_{\mathrm{rad}}\right)^{3 / 4}$, at the decay time of $\phi$ is calculated, and the primordial baryon-to-entropy ratio is multiplied by $D^{-1}$ to obtain the resulting baryon asymmetry.

Fig. 4.3 shows the $\left|\phi_{0}\right|$ dependence of the present baryon-to-entropy ratio, $n_{B} / s$. From the figure, one can see that the results based on the order of magnitude estimations provide good approximations. For a sufficiently large $\phi_{0}$ such that the entropy production is significant, the resultant baryon-to-entropy ratio is independent of the gravitino mass, and is proportional to $\left|\phi_{0}\right|^{2}$. It was also checked that the approximate formula (4.5.15) reproduces the behavior for the large $\left|\phi_{0}\right|$ region. For a smaller value of $\left|\phi_{0}\right| \lesssim 10^{14} \mathrm{GeV}$, the entropy production from the decay becomes negligible. The result then is proportional to $\left|\phi_{0}\right|^{4}$ (Eq. (4.5.8)). For an even smaller $\left|\phi_{0}\right| \lesssim \phi_{\mathrm{eq}} \sim 7 \times 10^{11} \mathrm{GeV} \times\left(100 \mathrm{keV} / m_{3 / 2}\right)$, the behavior goes over to $\left|\phi_{0}\right|^{9 / 2}$ (Eq. (4.5.18)). As noted in the paragraph below Eq. (4.5.18), there is a transition region from $\sim 10 \phi_{\mathrm{eq}}$ to $\phi_{\mathrm{eq}}$, where the curves fall less steeply because a logarithmic enhancement factor comes in. In any case, the baryon-to-entropy can clearly be sufficiently large in this scenario, as required by the standard big-bang nucleosynthesis $n_{B} / s \sim 10^{-10}$, if the initial amplitude is larger than $10^{13-14} \mathrm{GeV}$.

A more precise estimate of the baryon asymmetry requires the specification of the flat direction, the relevant operator, and the size of the initial amplitude. The usual caveat concerning the $B-L$ invariance applies: If one employs an operator 


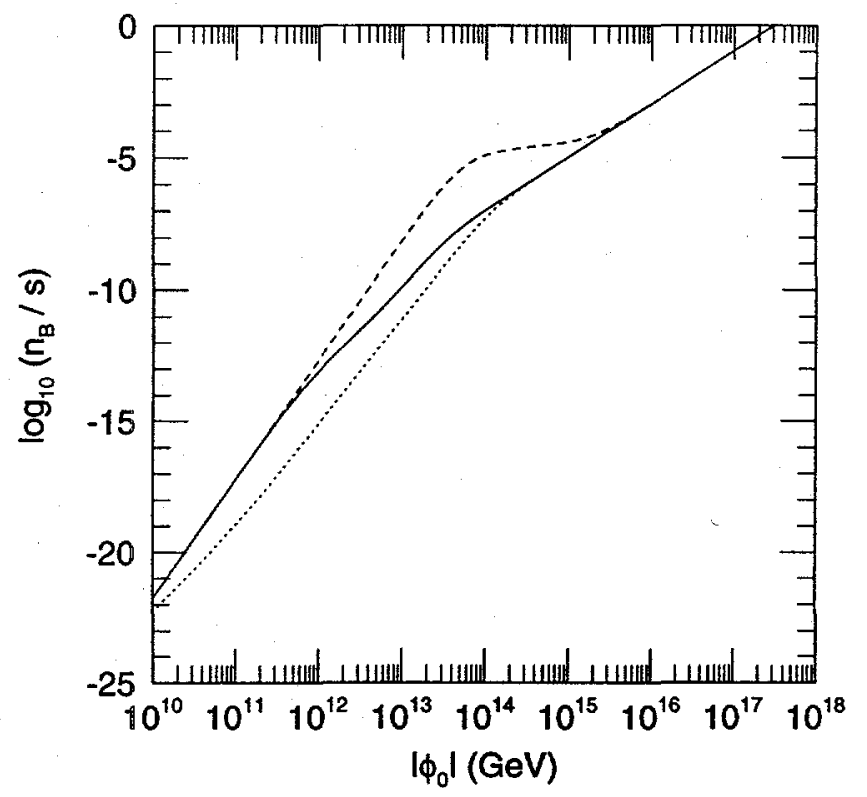

Figure 4.3: The resulting baryon-to-entropy ratio as a function of the initial amplitude $\phi_{0}$. The parameters are taken to be $V_{0}=\left(3 \times 10^{3} \mathrm{GeV}\right)^{4}, \theta_{0}=\pi / 8, \phi_{\mathrm{dec}}=10^{5}$ $\mathrm{GeV}$, and $m_{3 / 2}=1 \mathrm{keV}$ (dotted line), $m_{3 / 2}=100 \mathrm{keV}$ (solid line), and $m_{3 / 2}=10$ $\mathrm{MeV}$ (dashed line). 
which preserves $B-L$ symmetry like that generated by $S U(5)$ grand unified models, it may be wiped out again by the electroweak sphaleron effect [94]. One needs to either preserve the $B$ and $L$ asymmetries using a Bose condensate [93], or by generating a $B-L$ asymmetry $[95,96]$. In view of the discussions of the next section, we find that the protection of $B$ via a Bose condensate is a likely scenario.

\subsubsection{Diluting Gravitinos}

In the LEGM models, the mass of the gravitino is about $100 \mathrm{keV}$, and its mass density exceeds the closure limit if $T_{\max }$ is larger than $(1-10) \mathrm{TeV}$, as discussed in Sec. 4.2. Since the reheating after the primordial inflation raises the temperature typically above $T_{\mathrm{RH}} \gtrsim 10^{8} \mathrm{GeV}$ or so, it is assumed that the gravitinos were once thermalized, which is of course the worse case scenario. It will be discussed whether the decay of the flat direction can generate a large enough entropy to dilute gravitinos below the closure limit.

Before discussing the implication of the entropy production to the gravitino, it is useful to estimate the freeze-out temperature of the gravitino, $T_{\text {freeze }}$, which is the temperature at which the expansion rate of the Universe becomes comprable to the production rate of the gravitino: $H\left(T_{\text {freeze }}\right) \sim \Sigma_{\text {tot }} n_{\text {rad }}\left(T_{\text {freeze }}\right)$. By using the gravitino production cross section given in Eq. (4.2.5), one obtains

$$
T_{\text {freeze }} \sim 200 \mathrm{TeV} \times\left(\frac{m_{3 / 2}}{100 \mathrm{keV}}\right)^{2}\left(\frac{m_{\mathrm{G} 3}}{1 \mathrm{TeV}}\right)^{-2} .
$$


Below $T_{\text {freze }}$, the expansion rate of the Universe becomes larger than the production rate of the gravitino, and hence the gravitino cannot be thermalized. Therefore, an entropy production at $T \lesssim T_{\text {freeze }}$ dilutes gravitinos produced before the entropy production. On the contrary, even if the entropy is produced when $T \gtrsim T_{\text {freeze }}$, the gravitino is thermalized again, and its number density is determined by the thermal distributions. If the energy density of the flat direction dominates the energy density of the Universe, decay of the flat direction $\phi$ reheats the Universe. The reheating temperature is estimated as $T_{\mathrm{R}} \sim\left(V_{0}\right)^{1 / 4} \sim 1-10$ $\mathrm{TeV}$. Comparing this reheating temperature to Eq. (4.5.23), one can see that the gravitino cannot be thermalized after the decay of $\phi$. In other words, gravitinos produced before the decay of $\phi$ are diluted with a dilution factor given in Eq. (4.5.14), if the decay of $\phi$ produces the entropy.

If the gravitino mass is larger than $\sim 1 \mathrm{keV}$, a substantial entropy production is needed; otherwise, the Universe is overclosed by the mass density of the gravitino. By assuming that the gravitino is thermalized, one can estimate the number density of the gravitino as

$$
n_{3 / 2}(T)=\frac{3}{2} \frac{g_{*}(T)}{g_{*}\left(T_{\text {freeze }}\right)} n_{\mathrm{rad}}(T)
$$

Then, requiring $\Omega_{3 / 2}=D^{-1} m_{3 / 2} n_{3 / 2} / \rho_{\mathrm{c}} \leq 1$, one obtains

$$
D \gtrsim 50 \times h^{-2}\left(\frac{m_{3 / 2}}{100 \mathrm{keV}}\right) .
$$

Comparing the above constraint with Eq. (4.5.14), one can see that the decay of the flat direction can produce enough entropy to dilute the gravitinos away. 
For example, for $\phi_{\text {dec }} \sim 10^{5} \mathrm{GeV}$ and $m_{3 / 2} \sim 100 \mathrm{keV}$, the dilution factor is large enough, if $\left|\phi_{0}\right| \gtrsim 10^{14-15} \mathrm{GeV}$. Even with such a large dilution, the present baryon-to-entropy ratio can be sufficiently large (see Eq. (4.5.15)).

If the flat direction decays at an amplitude larger than $\sim 10^{5} \mathrm{GeV}$, the dilution factor given in Eq. (4.5.14) becomes smaller, and the entropy production due to the decay of $\phi$ may not be enough to decrease the gravitino density. In addition, for $m_{3 / 2} \lesssim 100 \mathrm{keV}$, the reheating temperature $T_{\mathrm{R}} \sim V_{0}^{-1 / 4} \sim 1-10 \mathrm{TeV}$ may be higher than the freeze-out temperature of the gravitino. In these cases, one has to assume an extra source of the entropy production of $O\left(m_{3 / 2} / 2 h^{2} \mathrm{keV}\right)$ after the reheating. Even in this case, the estimation of the primordial baryon-to-entropy ratio (4.5.8) is still valid, and the final baryon asymmetry can be as large as the one estimated by Eq. (4.5.15) and an additional dilution factor Eq. (4.5.25) required to dilute the gravitinos. Therefore, Affleck-Dine baryogenesis can generate a big enough baryon asymmetry to explain the present value of the baryon-to-entropy ratio.

In fact, one can crudely estimate the decay amplitude $\phi_{\mathrm{dec}}$ even when it is in the region of the logarithmic potential, if its motion is circular rather than elliptic. The change from the original Affleck-Dine estimate of the decay rate Eq. (E.7) is that the rotation frequency of the $\phi$ field is given by $\left(V_{0} \ln \left(|\phi|^{2} /\langle S\rangle^{2}\right)\right)^{1 / 2} /|\phi|$ rather than $m_{3 / 2}$. Since the quantity of interest is a dilution factor, it is assumed 
that the flat direction dominates the Universe, and the field decays when

$$
\Gamma_{\phi} \sim\left(\frac{\alpha_{s}}{\pi}\right)^{2} \frac{1}{\left|\phi_{\mathrm{dec}}\right|^{2}}\left(\frac{V_{0} \ln \left(\left|\phi_{\mathrm{dec}}\right|^{2} /\langle S\rangle^{2}\right)}{\left|\phi_{\mathrm{dec}}\right|^{2}}\right)^{3 / 2} \sim H \sim \frac{V_{0}^{1 / 2}}{M_{*}} \ln \frac{\left|\phi_{\mathrm{dec}}\right|^{2}}{\langle S\rangle^{2}}
$$

and

$$
\begin{aligned}
\left|\phi_{\mathrm{dec}}\right| & \sim\left[\left(\frac{\alpha_{s}}{\pi}\right)^{2} V_{0} M_{*}\left(\ln \frac{\left|\phi_{\mathrm{dec}}\right|^{2}}{\langle S\rangle^{2}}\right)^{1 / 2}\right]^{1 / 5} \\
& \sim 8 \times 10^{5} \mathrm{GeV} \times\left(\frac{V_{0}}{\left(3 \times 10^{3} \mathrm{GeV}\right)^{4}}\right)^{1 / 5} .
\end{aligned}
$$

Therefore, the decay amplitude does not change much from the value assumed before. On the other hand, the case with an elliptic orbit is more difficult to deal with. We are not aware of any study on the decay rate of $\phi$ for an arbitrary elliptic motion even for the parabolic potential. The other limit of almost linear motion is discussed in the literature and tends to give a larger decay rate, and hence a larger $\phi_{\text {dec }}$ [97]. However, we believe the motion of the $\phi$ field in the case of our interest here, namely for $\left|\phi_{0}\right| \gtrsim 10^{14-15} \mathrm{GeV}$ to dilute gravitinos, to be quite far from a linear one, and $\phi_{\text {dec }}$ is not likely to be much larger than our estimate. One concludes that $\phi_{\mathrm{dec}}$ is not much larger than the minimum possible value $10^{5} \mathrm{GeV}$, which is used in most of the present discussions.

\subsection{Cosmology of String Moduli}

It is pointed out in this section that the moduli fields in the string theory, if they acquire masses in the LEGM models, are stable and drastically overclose the Universe. 
According to a general analysis [98], string moduli acquire masses comparable to the gravitino mass $m_{3 / 2}$. Their initial amplitude is likely to be of the order of the string or Planck scale because it is the only scale in the problem. The cosmological problem of the moduli fields is discussed extensively in the literature in the context of hidden sector models (for the original chapter, see [99]). There, the moduli fields acquire masses of the order of $1 \mathrm{TeV}$, and decay after nucleosynthesis, thereby spoiling the success of the nucleosynthesis theory. Even if one pushes the mass to $10 \mathrm{TeV}$ so that the moduli fields decay before nucleosynthesis, the enormous production of entropy with a dilution factor of order $M_{*} / m_{3 / 2} \sim 10^{14}$ wipes out all pre-existing baryon asymmetry. This problem may be solved by adopting the Affleck-Dine baryogenesis [100], or by the thermal inflation [81] (see Appendix E for more discussions).

In the LEGM models, the situation is completely different.* The string moduli are stable within the cosmological time scale, and are still oscillating around their potential minima. A dimensional analysis gives the decay rate of a moduli field to be $\Gamma \sim m_{3 / 2}^{3} / 8 \pi M_{*}^{2} \sim\left(3 \times 10^{18} \text { years }\right)^{-1}$, for $m_{3 / 2} \sim 100 \mathrm{keV}$. Therefore there is a problem concerning its energy density.

The estimation of the moduli energy density is straight-forward. When a moduli field begins to oscillate, the expansion rate is $H \sim m_{3 / 2}$. The entropy at

*It was argued that the problem does not exist [101] if SUSY is broken dynamically, which is true for scalar fields which directly participate in the dynamical SUSY breaking. However, the string moduli fields were not considered in this discussion. 
this stage is given by $s \sim g_{*}^{1 / 4}\left(m_{3 / 2} M_{*}\right)^{3 / 2}$. Assuming the initial amplitude to be of order $M_{*}$, the ratio of the moduli energy density to the entropy is given by

$$
\frac{\rho_{\text {moduli }}}{s} \sim g_{*}^{-1 / 4}\left(m_{3 / 2} M_{*}\right)^{1 / 2} \sim 1.3 \times 10^{6} \mathrm{GeV}\left(\frac{m_{3 / 2}}{100 \mathrm{keV}}\right)^{1 / 2} .
$$

Since both the energy density of the moduli and the entropy are diluted by the expansion with the same rate $R^{-3}$, the ratio remains constant until now unless there is entropy production. On the other hand, the total energy density is bounded from above by the critical density $\rho_{c}$,

$$
\frac{\rho_{\text {moduli }}}{s} \leq \frac{\rho_{c}}{s_{\text {now }}}=3.6 \times 10^{-9} h^{2} \mathrm{GeV}
$$

where $s_{\text {now }}$ is the present value of the entropy density. The predicted ratio Eq. (4.6.1) is in gross conflict with the constraint Eq. (4.6.2).

It is not clear how such an enormous energy density can be diluted. First of all, the necessary dilution factor is at least $10^{15}$. Furthermore, one needs such an entropy production at a very late stage of the Universe, with $H \leq m_{3 / 2}$, or equivalently, $T \leq g_{*}^{-1 / 4}\left(m_{3 / 2} M_{*}\right)^{1 / 2}=1.3 \times 10^{6} \mathrm{GeV}$ for $m_{3 / 2}=100 \mathrm{keV}$. One needs to create a baryon asymmetry either after such an enormous entropy production at a very late stage, or large enough to survive the enormous entropy production.

Actually, the Affleck-Dine mechanism may create a large enough baryon asymmetry to survive the enormous entropy production which dilutes the string moduli below the critical density as will be shown below.

A quantity which remains constant over an entropy production is the ratio of 
the baryon number and the moduli energy density, because both of them scale as $R^{-3}$. In the previous section, it was estimated that the initial baryon number density is $n_{B} \sim m_{3 / 2} \operatorname{Im} \phi_{0}^{4} / M_{*}^{2}$, at the time when the flat direction begins to oscillate, i.e., $H \sim m_{3 / 2}$. In fact, this is the same time as when the moduli fields begin to oscillate, and the energy density of the moduli is $\rho_{\text {moduli }} \sim m_{3 / 2}^{2} M_{*}^{2}$. Therefore, their ratio is determined at this stage:

$$
\frac{\rho_{\text {moduli }}}{n_{B}} \sim m_{3 / 2}\left(\operatorname{Im} \frac{M_{*}^{4}}{\phi_{0}^{4}}\right)
$$

or equivalently,

$$
\frac{n_{B}}{s} \sim \frac{\rho_{\text {moduli }}}{s} \times m_{3 / 2}^{-1}\left(\frac{\left|\phi_{0}\right|}{M_{*}}\right)^{4} \sin 4 \theta_{0}
$$

Combining the above equation with the constraint (4.6.2), one finds

$$
\frac{n_{B}}{s} \lesssim \frac{\rho_{c}}{s_{\text {now }}} \times m_{3 / 2}^{-1}\left(\frac{\left|\phi_{0}\right|}{M_{*}}\right)^{4} \sin 4 \theta_{0} \sim 4 \times 10^{-5} h^{2}\left(\frac{m_{3 / 2}}{100 \mathrm{keV}}\right)^{-1}\left(\frac{\left|\phi_{0}\right|}{M_{*}}\right)^{4} \sin 4 \theta_{0}
$$

As one can see, if $\left|\phi_{0}\right| \gtrsim 10^{17} \mathrm{GeV}$, the baryon-to-entropy ratio may be larger than $\sim 10^{-10}$ even if one assumes a large entropy production to dilute the moduli field. The important question is whether one can have a brief period of inflation at such a late stage of Universe to dilute string moduli in the LEGM models. The inflationary expansion rate $H_{\text {inf }}$ must be less than $H_{\text {inf }} \lesssim m_{3 / 2} \sim 100 \mathrm{keV}$, i.e. the energy density of the inflation $\rho_{\text {inf }} \lesssim\left(m_{3 / 2} M_{*}\right)^{2} \sim\left(10^{7} \mathrm{GeV}\right)^{2}$. Moreover, the $e$-folding should not exceed 20 or so in order to keep the primordial density fluctuation generated by a "standard" inflation with $H_{\text {inf }} \sim 10^{11}-10^{13} \mathrm{GeV}[103]$. 
On the other hand, an $e$-folding of $N \gtrsim 5$ is sufficient to dilute the string moduli by $10^{-15}$. A thermal inflation [81] may offer a natural solution to these questions.

Fortunately, it seems to be unnecessary to introduce new energy scales into the model in the framework of the thermal inflation. Suppose a positive mass squared of $m^{2} \sim(100 \mathrm{GeV})^{2}$ is generated for a scalar field $\chi$ due to higher order loops at the energy scale of $\Lambda_{\mathrm{DSB}} \sim 10^{7} \mathrm{GeV}$. The renormalization group running of the mass squared may drive it negative at a scale slightly below $\Lambda_{\mathrm{DSB}}$. If the scalar field is a flat direction of both $F$ - and $D$-terms in the potential, it develops a minimum at $v \lesssim \Lambda_{\text {DSB }}$. This is an ideal potential for a thermal inflation. The scalar field may initially be stuck at the origin because of the thermal effects, giving a cosmological constant. As the radiation gets red-shifted, the thermal effects turn off and the field rolls down the potential to its true minimum $\chi=v$. The $e$-folding in this case is roughly $N \simeq \frac{1}{2} \ln (v / m) \sim \frac{1}{2} \ln \left(\Lambda_{\mathrm{DSB}} / m\right) \sim 5$ [81] which is exactly what is needed to dilute the string moduli below the critical density.

It may be interesting to compare this result with the case of the hidden sector SUSY breaking scenario with the Polonyi field or with the string moduli. (Hereafter, they will be called generically as "Polonyi fields". See Appendix E for the estimation of baryon asymmetry in this case.) Note that Eq. (4.6.4) is valid. Thus, the question is the constraint on the energy density of the Polonyi field, $\rho_{z}$. In this case, the Polonyi field decays much faster than the LEGM case since its 
mass is larger, and a typical lifetime for the Polonyi field is given by

$$
\tau_{z} \sim\left(\frac{N_{\mathrm{ch}}}{4 \pi} \frac{m_{3 / 2}^{3}}{M_{*}^{2}}\right)^{-1} \sim 10^{3} \mathrm{sec} \times\left(\frac{m_{3 / 2}}{1 \mathrm{TeV}}\right)^{3}
$$

where $N_{\mathrm{ch}} \sim O(10)$ is the number of the decay channel. Thus, it does not contribute to the mass density of the present Universe, and the constraint (4.6.2) cannot be applied. However, it may affect the great success of the standard bigbang nucleosynthesis (BBN) scenario.

The mass density of the Polonyi field speeds up the expansion rate of the Universe when the neutron decouples from the thermal bath (i.e., $T \sim 1 \mathrm{MeV}$ ), which may result in an over production of ${ }^{4} \mathrm{He}$. Furthermore, the radiative decay of the Polonyi field induces cascade photons which cause the photofission process and change the primordial abundances of the light nuclei. The constraint on the primordial density of the Polonyi field strongly depends on its lifetime $\tau_{z}$ [102]. If $\tau_{z} \lesssim 10^{4} \mathrm{sec}$, nucleosynthesis requires $\rho_{z} / s \lesssim 10^{-5} \mathrm{GeV}$. For a Polonyi field with a longer lifetime, the constraint becomes more stringent. For a Polonyi field with $\tau_{z} \lesssim 10^{(4-5)}$ sec, which is the case for the Polonyi mass typically larger than a few $\mathrm{TeV}$, its mass density is constrained as $\rho_{z} / s \lesssim 10^{-7} \mathrm{GeV}$. These constraints on $\rho_{z} / s$ are compared to the estimate of the baryon-to-entropy ratio (E.14) which holds irrespective of the presence of a substantial dilution of Polonyi field by, e.g., a late inflation,

$$
\frac{n_{B}}{s} \sim \frac{\rho_{z}}{s} \times m_{3 / 2}^{-1}\left(\frac{\left|\phi_{0}\right|}{M_{*}}\right)^{4} \sin 4 \theta_{0}
$$

Thus, for this range of the Polonyi mass, the resulting baryon-to-entropy ratio 
may still be as large as $10^{-10}$ if $\left|\phi_{0}\right| \sim M_{*}$, and hence the Affleck-Dine scenario may provide us a reasonable value for the baryon-to-entropy ratio. However, if the Polonyi field has a longer lifetime, as for a sub- $\mathrm{TeV}$ Polonyi mass as usually expected, the constraint on $\rho_{z}$ becomes even more stringent. In particular, for the case with $\tau_{z} \gtrsim 10^{7} \mathrm{sec}$, which typically corresponds to $m_{z} \lesssim 100 \mathrm{GeV}, \rho_{z} / s \lesssim$ $10^{-13} \mathrm{GeV}$. In this case, the result is too small to be identified as the present baryon asymmetry of the Universe.

\subsection{Conclusion}

The cosmology of the LEGM models was studied. First, the lower bound on the gravitino mass was estimated, and it was shown that the bound conflicts with the cosmological constraint if the primordial gravitino is not diluted. This fact indicates a huge entropy production at a relatively low temperature, and the conventional scenario of baryogenesis may not work well.

In this case, the Affleck-Dine baryogenesis is one interesting possibility. The size of the baryon number violating operators is much smaller than in the hidden sector models. However the flat direction begins to move at a much later stage which in turn increases the baryon number. The dilution factor due to the decay of flat direction also has a complicated dependence on parameters. After putting all the effects together, it was found that the Affleck-Dine baryogenesis works efficiently for an initial amplitude of the flat direction, $\left|\phi_{0}\right| \gtrsim 10^{13} \mathrm{GeV}$. It was also discussed that the decay of the MSSM flat direction may provide enough 
entropy to dilute the primordial gravitino for a relatively large initial amplitude of the flat direction, $\left|\phi_{0}\right| \gtrsim 10^{14-15} \mathrm{GeV}$. Therefore, the gravitino problem in the LEGM models may be solved if one assumes such a large initial amplitude.

The cosmological implication of the moduli fields in string theory was also discussed. Their masses are of the order of the gravitino mass, and their lifetime is much larger than the present age of the Universe in the LEGM models. The mass density of the moduli field may overclose the Universe. To dilute the moduli fields, a very late inflation is needed. It was shown that the baryon asymmetry generated by Affleck-Dine baryogenesis can be large enough to survive such a late inflation for $\left|\phi_{0}\right| \gtrsim 10^{17} \mathrm{GeV}$, even if one assumes a huge entropy production to dilute the primordial moduli field below the critical density. 


\section{Chapter 5}

\section{The $\mu$-Problem and the Next-to-Minimal SSM}

\subsection{Introduction}

The primary motivation for supersymmetry (SUSY) is to stabilize the smallness of the electroweak scale against radiative corrections $[106,107,108]$, which can be as large as the Planck scale if the Higgs bosons are truly elementary. Once the electroweak scale is set in the tree-level Lagrangian, it only receives logarithmic radiative corrections, and hence its order of magnitude is not changed. Moreover, the electroweak symmetry remains unbroken in the Minimal Supersymmetric Standard Model (MSSM) in the absence of explicit SUSY-breaking parameters. Therefore, one can view the electroweak symmetry breaking as being triggered by the soft SUSY breaking. Indeed, the soft SUSY-breaking mass-squared of the Higgs boson can be driven negative due to the top quark loop [109] while all the other scalar bosons still have positive mass-squared. In this sense, there is nothing special about the Higgs boson. It is just one of many scalar bosons, which happens to acquire a negative mass-squared due to the top quark loop. This idea eliminates one of the least appealing features of the Standard Model. However, there are at least two open questions. First, SUSY by itself does not explain why the electroweak scale is small to begin with. Therefore, SUSY makes the smallness of the 
electroweak scale "technically natural," but not truly natural. Second, the MSSM contains one dimensionful parameter (the $\mu$-parameter), allowed by SUSY, in the superpotetial. The natural values of $\mu$ are either the Planck mass (the only natural dimensionful parameter available) or zero, but recent experimental constraints imposed by LEP2 imply that a nonzero $\mu \gtrsim 50 \mathrm{GeV}$ is required [110].

SUSY, fortunately, can potentially explain the smallness of the electroweak scale if it is broken dynamically [107]. The perturbative non-renormalization theorem forbids the generation of a mass scale in the superpotential if it is absent at the tree-level. However, non-perturbative effects can violate the nonrenormalization theorem, and a mass scale can be generated by dimensional transmutation: $\Lambda_{\text {SugY }} \sim M_{\text {Planck }} e^{-8 \pi^{2} / g^{2}\left|b_{0}\right|}$, if an asymptotically free gauge theory is responsible for SUSY breaking. There has been major progress in building models of dynamical SUSY breaking $[111,112,113,114,115,116,117]$, which became possible with the detailed understanding of the non-perturbative dynamics of SUSY gauge theories [118]. Furthermore, the so-called gauge mediation of SUSY breaking (GMSB) $[108,119]$ can generate soft SUSY-breaking parameters in the SUSY Standard Model in a phenomenologically desired form. Therefore, there is hope of understanding the smallness of the electroweak scale in a truly natural manner.

However, the other question remains largely unanswered: how can the dimensionful parameters in the superpotential naturally be of the order of the SUSYbreaking parameters? There have been extensive discussions on this subject in the literature, which are briefly summarized in Sec. 5.2. Unfortunately, many of the 
proposed mechanisms rely on either small parameters, accidental cancellations, or the absence of interactions allowed by symmetries. The current situation is found to be rather unsatisfactory.

A natural direction to follow is to start with a superpotential which does not contain a dimensionful parameter and hope that the electroweak scale is generated solely due to the soft SUSY-breaking parameters. The simplest model which can potentially work along this line is the Next-to-Minimal Supersymmetric Standard Model (NMSSM) [120], which replaces the $\mu$-parameter by the vacuum expectation value of an electroweak singlet superfield. This possibility is revisited with detailed quantitative studies in this chapter. Unfortunately, the conclusion reached here is negative. The NMSSM by itself does not produce a phenomenologically viable electroweak symmetry breaking even if one varies the messenger scale. The major experimental constraints include Higgs boson and slepton searches. Certain simple modifications can evade phenomenological constraints, but require a cancellation among parameters accurate to a few percent. All of these points are presented quantitatively in this chapter, and we hope that the results prompt further investigations in understanding the origin of the $\mu$-parameter in models with the GMSB.

The chapter is organized as follows. In the next section, the situation of the $\mu$-problem in models with the GMSB is reviewed, and various proposals to explain the origin of the $\mu$-parameter are discussed. None of them, however, are found to be entirely satisfactory. Even if one accepts one of the proposed models, it is 
still necessary to check whether the generated $\mu$-parameter is phenomenologically allowed. This question is addressed in Sec. 5.3, and it is determined that the currently available experimental lower bounds on superparticle masses already require a cancellation of order $10 \%$ between the $\mu$-parameter and soft SUSYbreaking parameters to reproduce the observed $M_{Z}$. Then, the quantitative results of electroweak symmetry breaking in the NMSSM with the GMSB are presented in Sec. 5.4, and it is found that there is no phenomenologically viable parameter set even if one varies the messenger scale from $10^{5}$ to $10^{16} \mathrm{GeV}$. Various simple modifications of the NMSSM are studied in Sec. 5.5, and it is shown that they either do not break electroweak symmetry in a phenomenologically viable manner or require a cancellation among parameters of order $1 \%$. Sec. 5.6 contains the conclusions.

\subsection{The $\mu$-problem in the GMSB}

In this section, the $\mu$-problem in the MSSM is reviewed, and various attempts to solve it in the context of the GMSB are presented.

The parameter $\mu$ is the only dimensionful quantity present in the superpotential of the MSSM

$$
W=\mu H_{u} H_{d}+\lambda_{i j}^{l} L_{i} e_{j} H_{d}+\lambda_{i j}^{d} Q_{i} d_{j} H_{d}+\lambda_{i j}^{u} Q_{i} u_{j} H_{u}
$$

Here, $Q_{i}, L_{i}, u_{i}, d_{i}, e_{i}$ are the matter chiral superfields with the obvious notation, and $H_{u}, H_{d}$ the Higgs doublets. Note that $\mu$ is part of the supersymmetric 
Lagrangian, and hence its origin is, naively, unrelated to the origin of the soft SUSY-breaking terms

$$
\begin{aligned}
V_{\text {soft }}= & m_{H_{d}}^{2}\left|H_{d}\right|^{2}+m_{H_{u}}^{2}\left|H_{u}\right|^{2} \\
& +m_{\tilde{Q}}^{2 i j} \tilde{Q}_{i}^{\dagger} \tilde{Q}_{j}+m_{\tilde{L}}^{2 i j} \tilde{L}_{i}^{\dagger} \tilde{L}_{j}+m_{\tilde{u}}^{2 i j} \tilde{u}_{i}^{\dagger} \tilde{u}_{j}+m_{\tilde{d}}^{2 i j} \tilde{d}_{i}^{\dagger} \tilde{d}_{j}+m_{\tilde{e}}^{2 i j} \tilde{e}_{i}^{\dagger} \tilde{e}_{j} \\
& -m_{3}^{2} H_{u} H_{d}+\mathcal{A}_{d}^{i j} \tilde{Q}_{i} \tilde{d}_{j} H_{d}+\mathcal{A}_{u}^{i j} \tilde{Q}_{i} \tilde{u}_{j} H_{u}+\mathcal{A}_{l}^{i j} \tilde{L}_{i} \tilde{e}_{j} H_{d} .
\end{aligned}
$$

Phenomenology, on the other hand, dictates that the values of both $\mu$ and the soft SUSY-breaking masses should be around the weak scale $(100 \mathrm{GeV})$, if SUSY is to be responsible for stabilizing the Higgs mass. Therefore, the important question is how the mechanism of SUSY breaking can induce a $\mu$-parameter naturally, at the same order of magnitude as the other soft SUSY-breaking parameters in the Lagrangian.

One popular scenario of SUSY breaking is the so-called "hidden sector" SUSY breaking in supergravity (SUGRA) [121]. In hidden sector models, SUSY is broken in the hidden sector by some mechanism, such as the Polonyi model [122], gaugino condensation [123], or the O'Rafeartaigh model [124], and the effects of SUSY breaking are mediated to the fields in the MSSM only by interactions suppressed by the Planck scale. It therefore requires SUSY breaking at a scale $\Lambda \sim 10^{10} \mathrm{GeV}$ if the soft SUSY-breaking masses are generated as $\Lambda^{2} / M_{\text {Planck }}$. This class of models is able to generate the appropriate soft SUSY-breaking masses and $\mu$-parameter given that the $\mu$-term is forbidden in the supersymmetric limit by appropriate symmetries, and arises due to SUSY breaking (see, for example, the Giudice- 
Masiero mechanism [125]). Hidden sector models have, on the other hand, to face serious bounds imposed by flavor-changing neutral currents (FCNC) [126]. Lowenergy constraints such as the smallness of $K^{0}-\overline{K^{0}}$ mixing require the matrices $m_{\tilde{Q}}^{2 i j}, m_{\tilde{d}}^{2 i j}$ to have eigenvalues degenerate to a few percent, or their eigenvectors to be strongly "aligned" with the eigenvectors of the Yukawa matrices $\lambda_{i j}^{d}$ (the same is true for $\mathcal{A}_{d}^{i j}$ ). Within the SUGRA framework alone, there is no natural mechanism to guarantee the degeneracy or the alignment [127]. In this case, flavor symmetries are probably necessary to ensure either degeneracy [128] or alignment [127] and suppress FCNC, and some of the models presented are also capable of generating the $\mu$-term through flavor symmetry breaking $[129,130]$. There is also the possibility that string theory generates degenerate squark masses if, for instance, the dilaton field provides the dominant contribution to the soft SUSYbreaking masses [131].

The gauge mediation of supersymmetry breaking is an alternative mechanism which can naturally ensure the degeneracy of squarks masses and therefore suppress the dangerous FCNC effects. SUSY is somehow broken (hopefully dynamically via dimensional transmutation to generate a large hierarchy), and SUSYbreaking effects are mediated to the fields in the supersymmetric Standard Model by the Standard Model gauge interactions. Mediating SUSY breaking via gauge interactions is not a novel idea $[108,119]$. It allows for SUSY breaking at a lower scale (when compared to SUGRA inspired models) and, because all SUSYbreaking effects are transmitted by flavor blind interactions (the Standard Model 
gauge interactions), squarks of different families have the same mass. This scheme has attracted a lot of interest after the pioneering works by the authors of references $[111,112,113]$, which showed that one can successfully mediate the SUSYbreaking effects via gauge interactions with the help of a so-called "messenger sector." Their scheme can easily incorporate dynamical SUSY breaking and can explain the origin of the large hierarchy between the Planck (string, grand unified (GUT)) scale and the weak scale.

The GMSB itself, however, has nothing to say about the $\mu$-parameter unless one introduces extra fields which couple to the particle content of the MSSM. The $\mu$-problem in the GMSB is the primary interest of this chapter. Many solutions to the $\mu$-problem have been suggested by different authors and all of them require the introduction of new fields and/or interactions. Some of these solutions will be reviewed shortly.

In the original models $[111,112,113]$, SUSY is broken dynamically in a so-called SUSY-breaking sector and the breaking effects are transmitted to the supersymmetric Standard Model via a messenger sector. The energy scale of the messenger sector is given by $\Lambda \simeq 10^{4}-10^{5} \mathrm{GeV}$. There are, however, models which do not have a separate messenger sector so that the sector which breaks SUSY dynamically is directly coupled to the Standard Model gauge group $[115,116,117]$. In this case, the effective messenger scale tends to be much higher. For the purposes of this chapter, it is enough to employ a simple version of the messenger sector, as in the original models, and take the messenger scale $\Lambda$ as a free parameter. 
The messenger sector can be described by the superpotential

$$
W_{S}=\frac{1}{3} \lambda S^{3}+\kappa S \Phi^{+} \Phi^{-}+\kappa_{q} S q \bar{q}+\kappa_{l} S l \bar{l}
$$

where $S$ is a singlet superfield, $\Phi^{ \pm}$are charged under a $U(1)$ associated with the SUSY-breaking sector and are singlets under the Standard Model $S U(3) \times S U(2) \times$ $U(1)$ gauge group. The superfield $q(\bar{q})$ transforms as a $(3(\overline{3}), 1, \pm 1 / 3)$ under the Standard Model, while $l(\bar{l})$ transforms as $(1,2, \mp 1 / 2)$.

It is assumed that the scalar components of $\Phi^{ \pm}$acquire negative SUSYbreaking masses-squared due to its interaction with the SUSY-breaking sector (usually accomplished by the so-called "messenger $U(1)$ " gauge interaction $[112,113])$, and the potential associated with the scalar component of $S$ reads

$$
V_{S}=-\left|m_{\Phi}\right|^{2}\left(\left|\Phi^{+}\right|^{2}+\left|\Phi^{-}\right|^{2}\right)+\left|\kappa S \Phi^{+}\right|^{2}+\left|\kappa S \Phi^{-}\right|^{2}+\left|\kappa \Phi^{+} \Phi^{-}+\lambda S^{2}\right|^{2}
$$

neglecting terms containing $l$ or $q$. It is easy to see that the scalar and the $F$ components of $S$ acquire vacuum expectation values (VEVs) $\langle S\rangle$ and $\left\langle F_{S}\right\rangle$ and therefore $q$ and $l$ acquire supersymmetric masses proportional to $\langle S\rangle$ and SUSYbreaking masses-squared proportional to $\left\langle F_{S}\right\rangle .^{*}$ This effect feeds down to the MSSM through loop corrections. Gauginos acquire Majorana masses at one loop, while sfermions acquire SUSY-breaking masses-squared at two loops. The calculation of these soft SUSY-breaking parameters was done long ago (see $[108,119])$

*There is a run-away direction $q=\bar{q}, l=\bar{l}$ in this potential [132]. This problem can be avoided by introducing more $S$ fields to the messenger sector. Such details are, however, irrelevant for the rest of this discussion. 
and its result is well known. At the messenger scale:

$$
\begin{gathered}
M_{i}=\frac{\alpha_{i}}{4 \pi} n B \\
m_{\tilde{f}_{i}}^{2}=2 n B^{2}\left(\frac{3}{5} Y_{i}^{2}\left(\frac{\alpha_{1}}{4 \pi}\right)^{2}+C_{2 i}\left(\frac{\alpha_{2}}{4 \pi}\right)^{2}+C_{3 i}\left(\frac{\alpha_{3}}{4 \pi}\right)^{2}\right) .
\end{gathered}
$$

Here and below, all $\alpha_{i}=g_{i}^{2} / 4 \pi$ are in the $S U(5)$ normalization, $B=\left\langle F_{S}\right\rangle /\langle S\rangle$ in the messenger sector discussed above, and $n$ determines the number of messenger sector superfields responsible for mediating SUSY breaking. In the example described above, which will be referred to as the model with the minimal GMSB, $n=1 . \quad Y$ is the hypercharge of the particle, $C_{2}=3 / 4$ for weak $S U(2)$ doublets (zero for singlets) and $C_{3}=4 / 3$ for color triplets (zero for color singlets). Eq. (5.2.6) guarantees that squarks of different families are degenerate at the messenger scale and therefore FCNC effects are safely suppressed. It is interesting to note that, for small $n$, gaugino masses and sfermion masses are comparable. For very large $n$, on the other hand, sfermion masses can be significantly smaller than gaugino masses (by a factor $\sqrt{n}$ ).

In the mechanism described above, trilinear couplings are not generated at the same order (in loop expansion) at the messenger scale. This is not the case in general, and some models can generate trilinear couplings with values comparable to the other soft SUSY-breaking parameters even at the messenger scale [116]. For most of the discussions in this chapter,

$$
\mathcal{A}_{l, u, d}^{i j}(\Lambda)=0,
$$

will be considered, unless otherwise noted. 
The GMSB does not generate a $\mu$-term because of the non-renormalization theorem. Therefore $\mu$ is an input of the model, and, because it has dimensions of mass, its only nonzero natural value is $M_{P l a n c k}\left(M_{\text {string }}, M_{G U T}\right)$. This is clearly not allowed phenomenologically. The $\mu$-term must, therefore, be forbidden at the Planck scale (by, say, a $Z_{3}$ symmetry) and generated dynamically. Below, various attempts to generate the $\mu$-term in the context of the GMSB are reviewed. The following list is not meant to be exhaustive and the descriptions of the various attempts are by no means complete. The review below only intends to show that many attempts have been made while none of them appears to be entirely satisfactory.

The simplest solution would be to introduce a term in the superpotential [112]

$$
W \supset k S H_{d} H_{u}
$$

where $S$ is the singlet superfield in Eq. (5.2.3). In such a scenario $\mu=k\langle S\rangle$ and $m_{3}^{2}=k\left\langle F_{S}\right\rangle \cdot m_{3}^{2}$ is the SUSY-breaking Higgs mixing mass-squared in Eq. (5.2.2).

Phenomenology imposes that both $\mu$ and $\sqrt{m_{3}^{2}}$ are of the order of the weak scale, unless one is willing to accept a drastic cancellation among parameters to reproduce the observed $M_{Z}$. Therefore,

$$
\begin{aligned}
(k\langle S\rangle)^{2} \sim k\left\langle F_{S}\right\rangle & \sim(100 \mathrm{GeV})^{2}, \\
\frac{\left\langle F_{S}\right\rangle}{\langle S\rangle} \sim k\langle S\rangle & \sim 100 \mathrm{GeV}
\end{aligned}
$$

and

$$
m_{3}^{2}=\mu \frac{\left\langle F_{S}\right\rangle}{\langle S\rangle}
$$


This situation is already excluded experimentally. Eq. (5.2.5) states that the gluino mass is given by $\left(\alpha_{3} / 4 \pi\right)\left\langle F_{S}\right\rangle /\langle S\rangle$, and if Eq. (5.2.10) is satisfied one would arrive at $M_{\tilde{g}} \simeq 1 \mathrm{GeV}$, which is unacceptable. The same is true for all the other soft SUSY-breaking masses. This is a general consequence of Eq. (5.2.11). It implies that $\sqrt{m_{3}^{2}} \gg \mu$ if all experimental bounds on the SUSY spectrum are to be satisfied, while $S U(2) \times U(1)$ breaking requires Eq. (5.2.9). Some authors refer to this puzzle as the $\mu$-problem in the GMSB [133].

Another simple solution that does not require the introduction of any extra superfields into the theory couples the Higgs superfields to the $q$ superfields present in Eq. (5.2.3) [133]. In the minimal messenger sector $[112,113]$, one may have, instead of $q$ and $l$, a complete $\mathbf{5}+\overline{\mathbf{5}}$ multiplet of $S U(5)$ to preserve the gauge coupling unification. One can also use a $\mathbf{1 0}+\overline{\mathbf{1 0}}$ for this purpose, and generate gaugino masses and scalar masses-squared with $n=3$. In this case, one can couple the components $Q$ in $\mathbf{1 0}$ that have the same quantum numbers as left-handed quark doublets and the components $u$ that have the same quantum numbers as right-handed up quarks (or their corresponding components in $\overline{10}$ ) to the Higgs doublets. Explicitly, $W \supset \lambda_{1} H_{d} \bar{Q} \bar{u}+\lambda_{2} H_{u} Q u$. This will induce, in the Lagrangian, a one-loop term proportional to

$$
\frac{\lambda_{1} \lambda_{2}}{16 \pi^{2}} \int d^{4} \theta \frac{H_{d} H_{u} S^{\dagger} S^{\dagger}}{S^{\dagger} S}
$$

The $F$ vacuum expectation value of $S$ will generate $\mu \simeq \frac{\lambda_{1} \lambda_{2}}{16 \pi^{2}} \frac{\left\langle F_{S}\right\rangle}{\langle S\rangle}$ and $m_{3}^{2} \simeq$ $\frac{\lambda_{1} \lambda_{2}}{16 \pi^{2}}\left(\frac{\left\langle F_{S}\right\rangle}{\langle S\rangle}\right)^{2}$. Again one runs into Eq. (5.2.11) and must hunt for other solutions. 
All of the models described above couple the MSSM Higgs superfields to those in the messenger sector. Not only did one encounter the problem of Eq. (5.2.11), but some of the coupling constants introduced had to be made fairly small because of the magnitude of $\langle S\rangle$ and $\left\langle F_{S}\right\rangle$. Another class of solutions tries to get around this issue by introducing another singlet superfield, whose vacuum expectation value would generate the $\mu$-term.

One motivation for such models is to utilize the extra singlet to solve the doublet-triplet Higgs splitting in $S U(5)$ grand unified theories via a sliding singlet mechanism [134]. This mechanism is known to be unstable against radiative corrections if the soft SUSY-breaking parameters are generated at a scale higher than the GUT scale, but can be stable for the low-energy GMSB [135]. Ciafaloni and Pomarol [136] claim that such a solution would generate a viable $\mu$-term. We believe, however, that the conditions that they impose on the soft SUSY-breaking parameters can never be satisfied in the context of the GMSB, where all soft SUSY-breaking masses are tightly related. This will be discussed in Sec. 5.5.

The simplest model with the addition of an extra singlet one can imagine, referred to as the NMSSM [120], involves substituting the $\mu$-term in the MSSM superpotential by

$$
\lambda H_{d} H_{u} N-\frac{k}{3} N^{3} .
$$

The minimization of the scalar potential for $H_{d}, H_{u}$ and $N$ at the weak scale should produce VEVs $v_{d}$ and $v_{u}$ for both Higgs bosons, thus breaking $S U(2) \times$ $U(1)$, and $x$ for the singlet. $\mu$ would be equal to $\lambda x$. The $m_{3}^{2}$ term would arise 
due to renormalization group (RG) running of the $A$-term $\lambda A_{\lambda} H_{d} H_{u} N$ from the messenger scale to the weak scale. $m_{3}^{2}$ would be equal to $\lambda A_{\lambda} x$.

Dine and Nelson [111] claim that this model does not work for the low-energy GMSB. A detailed analysis was not presented in their papper, and the problem will be explained in Sec. 5.4. They suggest the introduction of an extra light pair of $q^{\prime}+\bar{q}^{\prime}$ and $l^{\prime}+\bar{l}^{\prime}$ as a means to produce a viable spectrum. They did not, however, publish a quantitative analysis of the model, and say nothing about its naturalness. Agashe and Graesser [137] study this scenario and show that there is indeed a solution, but it is fine-tuned. They present a possibility to ease the fine-tuning by employing many lepton-like messengers while keeping the number of quark-like messengers small. In Secs. 5.4 and 5.5, the case for both the highand low-energy GMSB is analyzed in great detail.

There are ways of giving $N$ a VEV which are not related to electroweak symmetry breaking. In Ref. [112] two mechanisms are introduced, neither of them very appealing, where the $N$ VEV is generated at the messenger scale. Namely,

$$
W \supset-\frac{1}{2} k_{S} N^{2} S
$$

or

$$
W \supset-k_{q} N q \bar{q}-k_{l} N l \bar{l}
$$

in addition to the NMSSM. $S, q$ and $l$ are the messenger sector superfields present in Eq. (5.2.3). 
In the case of Eq. (5.2.14), a potential

$$
V_{N}=\left|k N^{2}+k_{S}\langle S\rangle N\right|^{2}-k_{S} N^{2}\left\langle F_{S}\right\rangle
$$

is generated for $N$ in the presence of $\langle S\rangle$ and $\left\langle F_{S}\right\rangle$ VEVs. If one assumes $k_{S}$ to be small, $N$ develops a VEV $x=\sqrt{\frac{k_{S}\left(F_{S}\right)}{k^{2}}}$, and $\mu=\frac{\lambda}{k} \sqrt{k_{S}\left\langle F_{S}\right\rangle}$ assuming all other couplings to be of order one. It is easy to see, a posteriori, that $k_{S}$ must indeed be small if one is to generate a phenomenologically viable $\mu$. Unfortunately this case requires that the soft SUSY-breaking masses-squared $\sim\left(\alpha_{i} / 4 \pi\right)^{2}\left(\left\langle F_{S}\right\rangle /\langle S\rangle\right)^{2}$ and $\mu^{2} \sim k_{S}\left\langle F_{S}\right\rangle$ are accidentally of the same order of magnitude. ${ }^{\dagger}$

The superpotential coupling Eq. (5.2.15) would lead to a potential

$$
V_{N} \supset\left|k N^{2}\right|^{2}-k_{q} N \frac{1}{32 \pi^{2}} \frac{\left(\kappa_{q}\left\langle F_{S}\right\rangle\right)^{2}}{\kappa_{q}\langle S\rangle}-(l \leftrightarrow q)
$$

The linear terms in $N$ arise via tadpole one-loop diagrams involving $q$ 's and $l$ 's. This would lead to $x^{3}=\frac{k_{q}}{k^{2}} \frac{1}{32 \pi^{2}} \frac{\kappa_{q}\left\langle F_{S}\right\rangle^{2}}{\langle S\rangle}+(l \leftrightarrow q)$. Again $k_{q}$ and $k_{l}$ would have to be small. This solution still faces the problem of explaining why a term $N S^{2}$ is not present in the superpotential. Note that the presence of such a term would lead to an unacceptably large VEV for $N$. One may argue, however, that this is "technically natural" because the absence of a term in the superpotential is preserved by radiative corrections. An even more serious problem is the need to

\footnotetext{
${ }^{\dagger}$ Although $k_{S}$ has to be small, its smallness is natural in the sense of 't Hooft. It can be interpreted as being generated due to the breaking of some global symmetry, such as $N \rightarrow$ $e^{2 \pi i / 3} N$ and $H_{1,2} \rightarrow e^{2 \pi i / 3} H_{1,2}$, while $S$ is invariant. This type of symmetry would also explain the suppression of a term $N S^{2}$ in the superpotential, which would be of order $\left(k_{S}\right)^{2}$.
} 
suppress the kinetic mixing $\int d^{4} \theta S^{\dagger} N+$ h.c. to ensure $F_{N} \ll F_{S}$; an unacceptably large $m_{3}^{2}=\lambda F_{N}$ would be generated otherwise. An order unity kinetic mixing can be induced via radiative corrections between the ultraviolet cutoff, say the Planck scale, and the messenger scale, and the bare parameter has to be chosen very carefully so that the unwanted mixing term can be canceled at the messenger scale. This kinetic mixing can be forbidden if there are two sets of messenger fields and if the field $N$ couples off-diagonally, e.g., $W=N q_{1} \bar{q}_{2}$ etc [138]. Then the tadpole term mentioned above is also forbidden, but a negative mass squared for the $N$ field can be generated instead. This would lead to the NMSSM in a successful manner; again the parameters must be carefully chosen as in the NMSSM with extra light quark pairs (see Sec. 5.5).

Another solution with extra singlets, which points an interesting way around Eq. (5.2.11), was suggested by Dvali, Giudice and Pomarol [133]. Their idea is to generate the $\mu$-term via the following one-loop effective term in the Lagrangian:

$$
\int d^{4} \theta \frac{H_{d} H_{u} D^{\alpha} D_{\alpha}\left(S^{\dagger} S\right)}{\langle S\rangle^{3}}
$$

where $D_{\alpha}$ is the supersymmetric covariant derivative. This works because $D^{2}$ cancels $\theta^{2}$ in $S$, while leaving $\bar{\theta}^{2}$ in $S^{\dagger}$. Then the integral over $d \bar{\theta}^{2}$ can be done and the $\mu$-term is generated, while $m_{3}^{2}$ is not. The $m_{3}^{2}$ term would arise at higher loops, or via some other mechanism.

An explicit realization of this mechanism [133] is the following. Suppose a singlet field $N$ acquires a linear term $M^{2} N$ in the superpotential due to its coupling to 
the messenger sector. Then the superpotential $W=N\left(Y^{2}+H_{u} H_{d}-M^{2}\right)$ leads to a minimum with $\langle Y\rangle=M, N=0$. However, by further coupling $N$ to the messenger superfields, i.e. $N q \bar{q}$ etc, a one-loop diagram of messenger fields generates the operator $\frac{1}{16 \pi^{2}} \int d^{4} \theta N S^{\dagger}\left(S^{\dagger} S\right) /\left\langle S^{\dagger} S\right\rangle$, which contains $V \sim \frac{1}{16 \pi^{2}} N\left\langle F_{S}\right\rangle^{2} /\langle S\rangle$. Note that this is the same linear potential generated in the case of Eq. (5.2.15). This tadpole term induces a VEV for $N$ of order $\langle N\rangle \sim \frac{1}{16 \pi^{2}}\left\langle F_{S}\right\rangle^{2} /\langle S\rangle /\langle Y\rangle^{2}$ which is of the order of the weak scale if $\langle Y\rangle^{2}=M^{2} \sim\left\langle F_{S}\right\rangle$. The $Y$ field plays a crucial role: it slides to cancel the $F$-component VEV of $N$ before the tadpole is added and, after SUSY is broken, its VEV is shifted and leads to $\left\langle F_{N}\right\rangle=m_{3}^{2} \sim \mu^{2}$, as required by phenomenology. Note that the $\mu$-parameter obtained here can be understood as a consequence of the effective Lagrangian Eq. (5.2.18), which is generated upon integrating out $N$ and $Y$ before substituting the effect of the VEVs of $S$.

The necessary linear term $\left(M^{2} N\right)$ in the superpotential for $N$ can be easily generated by the kinetic mixing between $N$ and $S$ or also by other mechanisms, as pointed out in reference [139]. One apparent drawback of this realization is that one needs a set of new fields whose interactions are arranged in a rather special way. Furthermore one would expect the presence of a term proportional to $S H_{u} H_{d}$ in the superpotential. This happens because both $S$ and $N$ couple to the messengers, that is, $W \supset S q \bar{q}+N q \bar{q}$, and have, therefore, the same quantum numbers. It has already been argued that a coupling $S H_{u} H_{d}$ has to vanish (see Eq. (5.2.8)). Finally it is worthwhile to point out that this model also suffers from the cancellation problem present in the MSSM (see Sec. 5.3). 
Dine, Nelson, Nir and Shirman [113] suggest yet another way of generating a $\mu$ term with the introduction of an extra singlet. It was inspired by flavor symmetry models in [129], and resembles a modified version of the NMSSM + Eq. (5.2.14):

$$
W \supset \lambda_{n} \frac{N^{n+1}}{M_{P l}^{n}} H_{d} H_{u}+\lambda_{m} \frac{N^{m+3}}{M_{P l}^{m}}+\lambda_{p} \frac{N^{2+p}}{M_{P l}^{p}} S
$$

where $M_{P l}$ is the Planck mass. When $m=2, n=1$ and $p=2$, it is easy to check that $\mu \sim \lambda_{n} \sqrt{\left\langle F_{S}\right\rangle}$. The other couplings are assumed to be of order 1 . It is also easy to see that one would require a very small, carefully chosen coupling $\lambda_{n}$ in order to guarantee $\mu \sim 100 \mathrm{GeV}$. It is worth noting that this mechanism does not generate an $m_{3}^{2}$ term.

At last it is interesting to mention another interesting possibility, pointed out by Yanagida [140] and Nilles and Polonsky [141]. Their models utilize the accidental equality $\left(\Lambda_{\mathrm{DSB}} / M_{*}\right)^{1 / 3} \sim(\alpha / 4 \pi)^{2}$, where $\Lambda_{\mathrm{DSB}} \sim 10^{7} \mathrm{GeV}$ is the scale of dynamical SUSY breaking (DSB) in models with the low-energy GMSB and $M_{*}=M_{P l} / \sqrt{8 \pi}$ the reduced Planck mass. By introducing a new SUSYpreserving sector with strong gauge dynamics, Yanagida's model generates a VEV for the superpotential which cancels the cosmological constant from the DSB sector. The constant superpotential in turn generates a $\mu$-term of order $\Lambda_{\mathrm{DSB}}\left(\Lambda_{\mathrm{DSB}} / M_{*}\right)^{1 / 3} \sim(\alpha / 4 \pi)^{2} \Lambda_{\mathrm{DSB}} \sim 1 \mathrm{TeV}$. The phenomenology of this model is the same as the previous one (see Eq. (5.2.19)). The model by Nilles and Polonsky makes use of the Planck-scale suppressed Kähler potential, $\int d^{4} \theta N\left(z^{*} z\right) / M_{*}$, where $z$ is a chiral superfield in the DSB sector with an $F$-component VEV. This 
operator may be present at the tree-level, but may also be generated by gravitational effects. It generates a tadpole for the singlet $N: V=\left(\Lambda_{\mathrm{DSB}}^{4} / M_{*}\right) N$. Together with the $\frac{k}{3} N^{3}$ superpotential of the NMSSM, it generates a VEV for $N$ of order $\left.\langle N\rangle \sim\left(\Lambda_{\mathrm{DSB}}^{4} / M_{*}\right)\right)^{1 / 3}$. Even though these models generate the correct $\mu$-term of order the weak scale in the models with the low-energy GMSB, this would not work for the high-energy GMSB.

None of the mechanisms outlined above are entirely satisfactory. Most of them require a very specific choice of parameters and the introduction of extra matter at or slightly above the weak scale. Furthermore, most of them have not been studied quantitatively (see, however, Ref. [142]), and there is no guarantee that they indeed generate the correct electroweak symmetry breaking pattern and an experimentally viable spectrum. And last, but not least, there is no study of how natural such a solution is, given that a viable pattern of electroweak symmetry breaking can be generated.

It is, therefore, part of goal of this chapter to study the simplest of the models mentioned above in detail. Before that, it is important to review the status of electroweak symmetry breaking in the MSSM, where the $\mu$-term is introduced "by hand." It will be pointed out that, in the case of the GMSB, the current lower bounds on superparticle masses already require an order $10 \%$ cancellation between the $\mu$-parameter and the soft SUSY-breaking parameters. 


\subsection{The $\mu$-parameter in the MSSM}

Various proposals to generate the $\mu$-parameter in models with the GMSB were reviewed. This section contains a review of how electroweak symmetry breaking occurs in the MSSM, assuming that the $\mu$-parameter and $m_{3}^{2}$ are somehow generated. In particular, there is a need for an order $10 \%$ cancellation between $\mu$ parameter and soft SUSY-breaking parameters in models with the GMSB given the current experimental lower bounds on superparticle masses. Note that the case of the NMSSM is different because the $\mu$-parameter is generated together with electroweak symmetry breaking and hence the two problems cannot be clearly separated. This will be discussed in the next two sections.

The tree-level Higgs potential in the MSSM is given by

$$
\begin{aligned}
V= & m_{1}^{2}\left|H_{d}\right|^{2}+m_{2}^{2}\left|H_{u}\right|^{2}-m_{3}^{2}\left(H_{d} H_{u}+\text { c.c. }\right)+ \\
& +\frac{g_{2}^{2}}{8}\left(H_{d}^{\dagger} \vec{\sigma} H_{d}+H_{u}^{\dagger} \vec{\sigma} H_{u}\right)^{2}+\frac{g^{2}}{8}\left(\left|H_{d}\right|^{2}-\left|H_{u}\right|^{2}\right)^{2},
\end{aligned}
$$

where the mass parameters involve both the supersymmetric $\mu$-term and the soft SUSY-breaking terms,

$$
\begin{aligned}
& m_{1}^{2}=\mu^{2}+m_{H_{d}}^{2}, \\
& m_{2}^{2}=\mu^{2}+m_{H_{u}}^{2} .
\end{aligned}
$$

In the MSSM, one can show that the vacuum can always be gauge rotated to the following configuration

$$
H_{d}=\left(\begin{array}{c}
v_{d} \\
0
\end{array}\right), \quad H_{u}=\left(\begin{array}{c}
0 \\
v_{u}
\end{array}\right) .
$$


The two expectation values need to satisfy $v_{d}^{2}+v_{u}^{2}=v^{2}=(174 \mathrm{GeV})^{2}$ in order to reproduce the observed $M_{Z}$, and it is conventional to parametrize them by $v_{d}=v \cos \beta, v_{u}=v \sin \beta$. The minimization condition of the potential can be rewritten in the following form:

$$
\begin{aligned}
\frac{M_{Z}^{2}}{2} & =-\mu^{2}+\frac{m_{H_{d}}^{2}-m_{H_{u}}^{2} \tan ^{2} \beta}{\tan ^{2} \beta-1} \\
2 m_{3}^{2} & =\left(2 \mu^{2}+m_{H_{d}}^{2}+m_{H_{u}}^{2}\right) \sin 2 \beta .
\end{aligned}
$$

It has been claimed that electroweak symmetry breaking is natural in the MSSM because $m_{H_{u}}^{2}$ is easily driven negative due to the presence the top Yukawa coupling in its RG evolution. In models with the minimal GMSB such as the original ones in $[112,113]$, the boundary condition for the supersymmetry breaking parameters are given by Eqs. $(5.2 .5,5.2 .6,5.2 .7)$. A simple one-loop approximation is valid in the case of the low-energy GMSB because of the small logarithm between the messenger scale $\Lambda$ and the electroweak scale, and one finds

$$
m_{H_{u}}^{2}\left(M_{Z}\right) \simeq m_{H_{d}}^{2}\left(M_{Z}\right)-\frac{6}{16 \pi^{2}} h_{t}^{2} 2 m_{\tilde{t}}^{2} \log \frac{\Lambda}{M_{Z}}
$$

which is always negative.

The need for a cancellation between the $\mu$-parameter and soft SUSY-breaking masses can be seen as follows. Experimental constraints bound the superparticle masses from below, which hence set a lower limit for the ratio $B=\left\langle F_{S}\right\rangle /\langle S\rangle$. Therefore one finds that $\left|m_{H_{u}}^{2}\right|$ is bounded from below. On the other hand, in order for the observed $M_{Z}^{2}$ to be reproduced, the $\mu$-parameter is constrained by Eq. (5.3.5). For a moderately large $\tan \beta \gtrsim 2, m_{H_{d}}^{2}$ can be completely neglected 
and one finds

$$
\frac{M_{Z}^{2}}{2} \sim-\mu^{2}-m_{H_{u}}^{2}
$$

This equation requires a cancellation between $\mu^{2}$ and (negative) $m_{H_{u}}^{2}$ to reproduce $M_{Z}^{2} / 2 \sim(70 \mathrm{GeV})^{2}$ correctly. The degree of cancellation is given by $\left(M_{Z}^{2} / 2\right) / \mu^{2}$. $^{\ddagger}$

To determine the lower limit on $\left|m_{H_{u}}^{2}\right|$, a number of experimental constraints are considered [144]. One is that the gluino must be heavier than $190 \mathrm{GeV}$, which becomes stronger if the squarks have comparable masses. The second is that the right-handed selectron must be heavier than $80 \mathrm{GeV} .{ }^{\S}$ For large $\tan \beta$, the righthanded stau may become rather light; it is then required that $m_{\tilde{\tau}}>55 \mathrm{GeV}$ if it decays into tau and a neutralino or gravitino, and $m_{\tilde{\tau}}>73 \mathrm{GeV}$ if it does not decay inside the detector. The lightest chargino was also considered to be heavier than $63 \mathrm{GeV}$. The most recent lower bound on the chargino mass [110] is $m_{\chi^{+}} \gtrsim 67 \mathrm{GeV}$, which leaves the analysis virtually unchanged.

The case of the minimal low-energy GMSB with small $\tan \beta$ is discussed first, to make the argument clear. Here $\Lambda \sim 10^{5} \mathrm{GeV}$ is considered. The gluino mass

\footnotetext{
${ }^{\ddagger}$ The degree of cancellation is defined as follows: it is a percentage quantity that measures how much a given input parameter (in this case $\mu^{2}$ ) is free to vary before a given output parameter (in this case $M_{Z}^{2}$ ) changes significantly. Explicitly, the degree of cancellation is $\left(\mathrm{d}\left(\log M_{Z}^{2}\right) / \mathrm{d}\left(\log \mu^{2}\right)\right)^{-1}$. This definition corresponds to the inverse of the Barbieri-Giudice function [143].

${ }^{\S}$ This bound depends on the mass of the neutralino into which the selectron decays. However, since $\mu$ turns out to be large, it is a posteriori justified to assume that the lightest neutralino is almost pure bino. Then the GMSB predicts the relation between selectron and the bino masses, and hence one has a fairly reliable lower bound.
} 
constraint requires

$$
B>23 \mathrm{TeV}
$$

This bound itself is independent from the messenger scale. However, the gluino mass bound depends on the mass of the squarks, and it strengthens if the squark masses are comparable to the gluino mass. For the minimal low-energy GMSB, squarks are significantly heavier than the gluino and the bound above can be used. A more stringent constraint is derived from the requirement that the right-handed sleptons are heavier than $80 \mathrm{GeV}$. Including the one-loop renormalization group evolution and the $D$-term, one finds

$$
\begin{aligned}
m_{\tilde{e}}^{2} & =2 \frac{3}{5}\left(\frac{\alpha_{1}}{4 \pi}\right)^{2} B^{2}+\frac{2}{11}\left(\left(\frac{\alpha_{1}\left(M_{m e s s}\right)}{\alpha_{1}\left(M_{Z}\right)}\right)^{2}-1\right) M_{1}^{2}\left(M_{Z}\right)+M_{Z}^{2} \sin ^{2} \theta_{W} \cos 2 \beta \\
& =2.89 \times 10^{-6} B^{2}-0.232 M_{Z}^{2} \cos 2 \beta
\end{aligned}
$$

Therefore one finds

$$
B>39 \mathrm{TeV}
$$

for the most conservative case $\cos 2 \beta=-1$. With this lower bound one finds

$$
m_{\tilde{t}}^{2}>2 \frac{4}{3}\left(\frac{\alpha_{3}\left(M_{m e s s}\right)}{4 \pi}\right)^{2} B^{2}>(430 \mathrm{GeV})^{2}
$$

Using the one-loop running of $m_{H_{u}}^{2}$, one obtains

$$
m_{H_{u}}^{2}\left(M_{Z}\right)<-(260 \mathrm{GeV})^{2}
$$

and as a result of the minimization condition,

$$
\mu>250 \mathrm{GeV}
$$



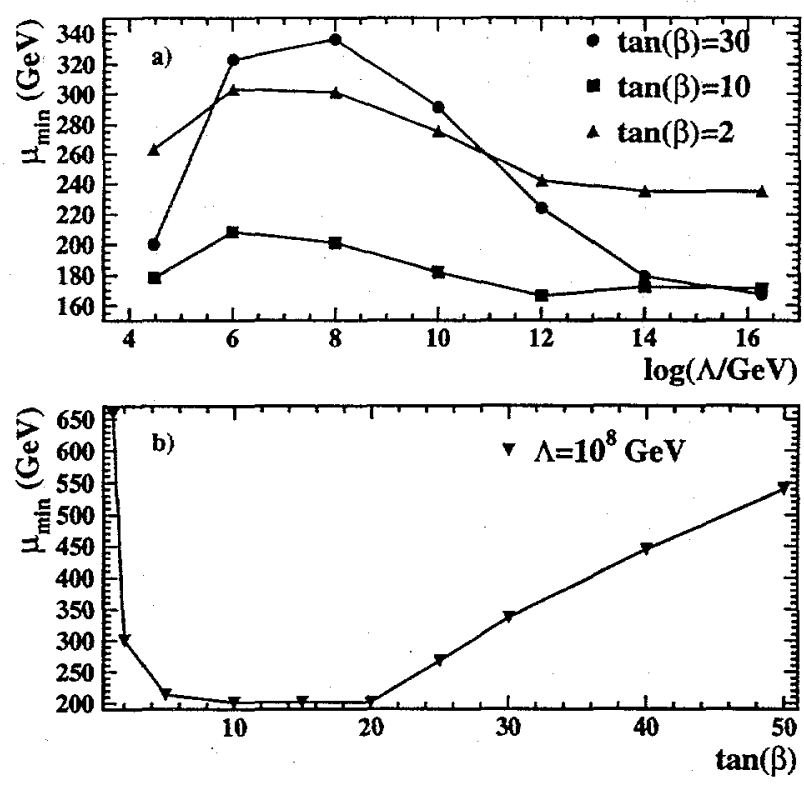

Figure 5.1: Lower bounds on $\mu$ in models with the GMSB subject to the constraint $M_{Z}=91 \mathrm{GeV}$ and to the lower bounds on superparticle masses (see text), (a) as a function of the messenger scale, for $\tan \beta=2,10$, and 30 , and (b) as a function of $\tan \beta$ for a fixed messenger scale of $10^{8} \mathrm{GeV}$.

This requires a cancellation of $7 \%$ in order to obtain the correct $M_{Z}^{2}$. Even though this level of cancellation is not of immediate concern, this analysis shows the need for a certain amount of cancellation which will become worse as experimental lower bounds on superparticle masses improve.

As it is clear from the argument above, the actual lower bound on $\mu$ depends on the messenger scale and $\tan \beta$. This issue was studied numerically using the experimental bounds quoted above and the lowest possible value of $\mu$ as a function of the messenger scale found. In Fig. 5.1(a) the bounds for three values of $\tan \beta$ 
are plotted. The lower bound on $\mu$ comes from one of the various experimental constraints. For instance, the $\tan \beta=2$ case is dominated by the lower bound on $m_{\tilde{e}_{R}}$ up to a messenger scale of $10^{12} \mathrm{GeV}$, after which the gluino mass bound is more important. The case of $\tan \beta=10$ has a similar behavior. The situation is more complex and interesting for $\tan \beta=30$. For a messenger scale of up to $10^{10} \mathrm{GeV}$, the stau is the lightest supersymmetric particle (except for the gravitino). It decays inside the detector to tau and gravitino for the lowest messenger scale, but leaves the detector without decaying for higher messenger scales. This stable stau provides the strongest constraint. From messenger scales above $\sim 10^{12} \mathrm{GeV}$ the stau decays inside the detector to tau and neutralino. This bound dominates up to $\sim 10^{16} \mathrm{GeV}$, when the gluino bound dominates. The chargino bound is comparable to that of the gluino for the GUT scale $\left(M_{G U T}=1.86 \times 10^{16} \mathrm{GeV}\right)$.

Fig. 5.1(b) depicts the minimum value of $\mu$ as a function of $\tan \beta$ for a fixed messenger scale $\left(\Lambda=10^{8} \mathrm{GeV}\right)$. The $\tan \beta$ dependence can be easily understood as follows. Starting from low $\tan \beta$, increasing $\tan \beta$ decreases the top Yukawa coupling, and hence $m_{H_{u}}^{2}$ receives a less negative contribution from the top-stop loop. Therefore a lower value of $\mu$ is allowed. This part is dominated by the $\tilde{e}_{R}$ bound. However beyond $\tan \beta \sim 20$, the bottom and tau Yukawa coupling become important. In fact, the scalar tau mass is pushed down both because of the loop effect and left-right mixing, and the experimental lower bound on $B$

\footnotetext{
TOnly the case of one messenger $(n=1)$ was analyzed. For larger $n$ the gluino bound becomes less important and the slepton bounds dominate up to the GUT scale.
} 
becomes stronger. Beyond $\tan \beta \sim 30$, the stau does not decay inside the detector for this choice of the messenger scale and the constraint is even more stringent. This in turn leads to a more negative $m_{H_{u}}^{2}$ and hence a larger $\mu$.

Combining both the messenger scale dependence and $\tan \beta$ dependence, one concludes that the most conservative current limit is

$$
\mu>160 \mathrm{GeV} \text {. }
$$

The required cancellation between $\mu^{2}$ and soft SUSY-breaking parameters in order to reproduce the observed $M_{Z}$ is $M_{Z}^{2} / 2 \mu^{2}=16 \%$. Note that this level of cancellation is the absolute minimum, and a more accurate cancellation is required for most of the parameter space.

In the case of minimal supergravity models, where all scalars have the universal SUSY-breaking mass-squared $m_{0}^{2}$, all gauginos have mass $M_{1 / 2}$ and all $A$-terms are given by $\mathcal{A}_{f}^{i j}=A_{0} \lambda_{f}^{i j}$ for $f=u, d, l$, at the GUT scale, the situation appears to be better. The renormalization group equations can be solved numerically for each choice of $\tan \beta$. As an example, for $\tan \beta=2$

$$
\begin{aligned}
m_{H_{1}}^{2} & =m_{0}^{2}+0.50 M_{1 / 2}^{2}, \\
m_{H_{2}}^{2} & =-0.32 m_{0}^{2}-2.49 M_{1 / 2}^{2}-0.05 A_{0}^{2}-0.20 M_{1 / 2} A_{0}, \\
m_{\tilde{e}}^{2} & =m_{0}^{2}+0.15 M_{1 / 2}^{2} .
\end{aligned}
$$

By requiring $m_{\tilde{e}}>80 \mathrm{GeV}$ and $M_{1 / 2}>60 \mathrm{GeV}$ (this is a rough bound inferred from the gluino bound $M_{\tilde{g}} \gtrsim 190 \mathrm{GeV}$ ), one finds $\mu>82 \mathrm{GeV}$. This basically does not require any cancellation, since $M_{Z}^{2} / 2 \mu^{2}=65 \%$. 
The situation can be somewhat ameliorated in the MSSM if there is a FayetIllioupoulos $D$-term for the $U(1)_{Y}$ gauge group. Such a $D$-term is known to arise in many ways, such as kinetic mixing of the $U(1)_{Y}$ and $U(1)_{\text {mess }}$ gauge fields [145]. The running of all the parameters remains the same except that one adds another contribution from the $D_{Y}$ at the weak scale (see Chap. 3 ). If the sign is appropriate, it increases $m_{\tilde{e}}^{2} \rightarrow m_{\tilde{e}}^{2}+D_{Y}$ and $m_{H_{u}}^{2} \rightarrow m_{H_{u}}^{2}+\frac{1}{2} D_{Y}$ (less negative) while decreasing $m_{H_{d}}^{2} \rightarrow m_{H_{d}}^{2}-\frac{1}{2} D_{Y}$. All of these help push the parameters relevant for electroweak symmetry breaking in the right direction. Larger $m_{\tilde{e}}^{2}$ reduces the lower bound on $\left\langle F_{S}\right\rangle /\langle S\rangle$, and a less negative $m_{H_{u}}^{2}$ is also welcome. Therefore the sensitivity to $\mu$ (required cancellation between $\mu$ and soft SUSYbreaking parameters) in the MSSM can be improved in the presence of a $D_{Y}$ with the appropriate sign.

It will be shown in the next two sections that the situation in the NMSSM is much worse. There is no phenomenologically viable solution to electroweak symmetry breaking. One can modify the model to generate a large negative masssquared for the singlet field and then find a viable solution. This solution also requires a cancellation among parameters which has the same origin as the cancellation present in the MSSM. It will also be shown that the addition of the Fayet-Illiopoulos $D$-term does not improve the situation within the NMSSM.

\subsection{The NMSSM with the GMSB}

In this section the feasibility of implementing the GMSB in the framework of 
the Next-to-Minimal Supersymmetric Standard Model (NMSSM) is studied. The presentation begins by introducing the NMSSM: its particle content, superpotential, and soft SUSY-breaking terms. The major steps in the analysis are briefly reviewed: the boundary conditions for the breaking terms, the RG evolution, and the minimization of the weak-scale one-loop effective potential. Then, the results of a numerical scan of a large portion of the model's parameter space are described. It is found that it is impossible to evade the present-day experimental constraints. This argument is further strengthened by providing a semi-analytical explanation for the inevitability of this conclusion.

\subsubsection{The NMSSM}

The NMSSM represents an attempt to solve the $\mu$-problem of the MSSM in the simplest and most direct way: the spectrum of the MSSM is augmented by a gauge singlet superfield $N$, which couples to $H_{d} H_{u}$ and plays the role of the $\mu$-term once it develops a nonzero vacuum expectation value [120]. The original $\mu$-term is banned from the theory so that there are no dimensionful parameters left in the superpotential.

The VEV of the scalar component of $N$ is determined by minimizing the scalar potential with respect to $H_{d}, H_{u}$, and $N$ simultaneously. It is natural to expect the VEVs to be of the same order of magnitude for all three fields, thus generating an effective $\mu$-parameter of order the weak scale, as required by phenomenology.

The complex scalar $N$ introduces two additional degrees of freedom to the Higgs 
sector. Therefore, the particle spectrum of the NMSSM contains three CP-even Higgs scalars, two CP-odd Higgs scalars, and one charged Higgs scalar. Immediately, there is a problem: one of the pseudoscalar Higgs bosons is massless. This happens because the superpotential $W=\lambda N H_{d} H_{u}$ has a Peccei-Quinn symmetry $N \rightarrow N e^{i \alpha}, H_{d} H_{u} \rightarrow H_{d} H_{u} e^{-i \alpha}$. This symmetry is spontaneously broken by the VEVs of the fields, making one of the pseudoscalars massless.

The standard solution to this problem is to introduce a term cubic in $N$, which explicitly breaks the symmetry mentioned above. This term is allowed by the gauge symmetries of the model and does not contain a dimensionful coupling constant, so it is generically expected to be present in the superpotential. One, however, still has to worry about a light pseudoscalar Higgs boson. As will be shown shortly, its mass can also be small because of the presence of a different (approximate) $U(1)$ symmetry.

Overall, the only change made to the MSSM superpotential is the following:

$$
\mu H_{d} H_{u} \longrightarrow \lambda N H_{d} H_{u}-\frac{k}{3} N^{3},
$$

while the corresponding change to the soft SUSY-breaking part of the potential is:

$$
-m_{3}^{2}\left(H_{d} H_{u}+\text { c.c. }\right) \longrightarrow-\left(\lambda A_{\lambda} N H_{d} H_{u}+\frac{1}{3} k A_{k} N^{3}+\text { h.c. }\right)+m_{N}^{2}|N|^{2} .
$$

One can determine the VEVs of the Higgs fields $H_{d}, H_{u}$, and $N$ by minimizing the scalar potential, which at the tree-level consists of the $F$-terms, $D$-terms, and 
soft SUSY-breaking terms:

$$
\begin{aligned}
V_{\text {Higgs }}^{\text {tree }}= & V_{F}+V_{D}+V_{\text {soft }}, \\
V_{F}= & \left|\lambda H_{d} H_{u}-k N^{2}\right|^{2}+\lambda^{2}|N|^{2}\left(\left|H_{d}\right|^{2}+\left|H_{u}\right|^{2}\right), \\
V_{D}= & \frac{g_{2}^{2}}{8}\left(H_{d}^{\dagger} \vec{\sigma} H_{d}+H_{u}^{\dagger} \vec{\sigma} H_{u}\right)^{2}+\frac{g^{2}}{8}\left(\left|H_{d}\right|^{2}-\left|H_{u}\right|^{2}\right)^{2}, \\
V_{\text {soft }}= & m_{H_{d}}^{2}\left|H_{d}\right|^{2}+m_{H_{u}}^{2}\left|H_{u}\right|^{2}+m_{N}^{2}|N|^{2}-\left(\lambda A_{\lambda} H_{d} H_{u} N+\text { h.c. }\right)- \\
& -\left(\frac{k}{3} A_{k} N^{3}+\text { h.c. }\right) .
\end{aligned}
$$

An important fact to notice is that both $V_{F}$ and $V_{D}$ remain unchanged when $H_{d}, H_{u}$, and $N$ are all rotated by the same phase. In fact, only the soft SUSYbreaking $A$-terms are not invariant under this transformation. This can be potentially dangerous, because, in general, the $A$-terms are considered to be zero at the messenger scale, and their sizes at the weak scale are determined by the RG evolution. If the generated values of $A_{\lambda}$ and $A_{k}$ are not large enough, the scalar potential has an approximate $U(1)$ symmetry. This symmetry is spontaneously broken by the vacuum expectation values of the Higgs fields, and, as before, one has to worry about a light pseudoscalar Higgs boson.

The VEVs of the neutral components of the Higgs fields are denoted by $v_{d}$ and $v_{u}$, as in Sec. 5.3, and the VEV of the singlet field by $x$ :

$$
\langle N\rangle=x .
$$

As a function of these VEVs, the potential has the form

$$
\begin{aligned}
V_{\text {neutral }}^{\text {tree }}= & \left|\lambda v_{d} v_{u}-k x^{2}\right|^{2}+\lambda^{2}|x|^{2}\left(\left|v_{d}\right|^{2}+\left|v_{u}\right|^{2}\right)+m_{H_{d}}^{2}\left|v_{d}\right|^{2}+m_{H_{u}}^{2}\left|v_{u}\right|^{2}+m_{N}^{2}|x|^{2}- \\
& -\left(\lambda A_{\lambda} v_{d} v_{u} x+\text { h.c. }\right)-\left(\frac{k}{3} A_{k} x^{3}+\text { h.c. }\right)+\frac{g_{2}^{2}+g^{\prime 2}}{8}\left(\left|v_{d}\right|^{2}-\left|v_{u}\right|^{2}\right)^{2} .
\end{aligned}
$$


It is well known that some of the Higgs boson masses receive significant contributions from radiative corrections. In the numerical analysis this is accounted for by employing the one-loop effective potential

$$
V_{\text {neutral }}^{1-\text { loop }}\left(v_{i}\right)=V_{\text {neutral }}^{\text {tree }}\left(v_{i}, \mu\right)+\frac{1}{64 \pi^{2}} \operatorname{STr} \mathcal{M}^{4}\left(v_{i}\right)\left(\log \frac{\mathcal{M}^{2}\left(v_{i}\right)}{\mu^{2}}-\frac{3}{2}\right)
$$

In this expression $\mathcal{M}^{2}\left(v_{i}\right)$ is a field-dependent scalar mass-squared matrix, and $\mu$ is the $\overline{M S}$ renormalization scale. As indicated explicitly, the values of the various parameters entering $V^{\text {tree }}$ depend on the choice of this scale. To the leading order this dependence is canceled when the second term on the right hand side of Eq. (5.4.6) is included, and the result of minimizing $V^{1-\text { loop }}$ is less sensitive to the choice of the scale where one stops running the RG equations. (Canceling out this dependence completely would require calculating radiative corrections to all orders.)

The matrix $\mathcal{M}^{2}$ depends on the field VEVs $v_{i}$ through the Yukawa couplings of the Higgs fields to various other particles. What plays a crucial role here is not the absolute values of the masses, but rather the rate of their change as one changes $v_{i}$. Therefore, the most important contribution comes from the field-dependent masses of the top quark and squarks, which have the largest Yukawa coupling. Denoting their mass eigenvalues by $m_{t}, m_{\tilde{t}_{1}}$ and $m_{\tilde{t}_{2}}$ respectively, the contribution to $V^{1-\text { loop }}$ from radiative corrections due to these states is 


$$
\begin{aligned}
& \Delta V=\frac{3}{32 \pi^{2}}\left[m_{\tilde{t}_{1}}^{4}\left(v_{i}\right)\left(\log \frac{m_{\tilde{t}_{1}}^{2}\left(v_{i}\right)}{\mu^{2}}-\frac{3}{2}\right)+m_{\tilde{t}_{2}}^{4}\left(v_{i}\right)\left(\log \frac{m_{\tilde{t}_{2}}^{2}\left(v_{i}\right)}{\mu^{2}}-\frac{3}{2}\right)\right. \\
& \left.-2 m_{t}^{4}\left(v_{i}\right)\left(\log \frac{m_{t}^{2}\left(v_{i}\right)}{\mu^{2}}-\frac{3}{2}\right)\right] .
\end{aligned}
$$

\subsubsection{Numerical Analysis}

In models with the GMSB the values of the soft SUSY-breaking terms are specified at the messenger scale by Eqs. (5.2.5), (5.2.6) and (5.2.7). Their values at the weak scale can be determined by solving the $R G$ equations given in Appendix $F$.

The model has five input parameters: $h_{t}, \lambda, k, B$, and $n$. (Note that the only dimensionful input parameter is $B$, and its magnitude will determine the overall scale of the VEVs and the soft SUSY-breaking masses.) There are, however, two constrains which must be satisfied at the weak scale: $v \equiv \sqrt{v_{d}^{2}+v_{u}^{2}}=174 \mathrm{GeV}$ and $h_{t} v_{u}=165 \pm 5 \mathrm{GeV} . \| \mathrm{A}$ common approach is to use the minimization conditions and RG equations to solve for the inputs, given a phenomenologically allowed set of weak-scale outputs. In the case of a high messenger scale, however, no easily invertible solution for the $R G$ equations is available. Instead, the problem is tackled numerically. After running down the RG equations and minimizing the Higgs potential once, this procedure is iterated, each time adjusting the value of the parameter $B$ to fix the overall scale of the VEVs and masses, while simultaneously

\footnotetext{
"Notice that this number is not equal to the top quark pole mass, the experimentally measured quantity, because of QCD corrections. The relationship between the two is given, at 1-loop, by $m_{\text {pole }}=\bar{m}\left(1+\frac{4}{3} \frac{\alpha_{0}}{\pi}\right)$.
} 
changing the dimensionless couplings to correctly reproduce the top quark mass. This iteration process, in fact, converges fairly quickly.

Using the procedure above, a numerical scan of a large portion of the parameter space is performed. The low-energy particle spectrum is studied for various messenger scales $\Lambda$, numbers of messengers $n$, and values of the couplings $\lambda$ and $k$. It is interesting to note that it is very easy to generate non-zero VEVs for $H_{d}, H_{u}$, and $N$, even when $m_{N}^{2}$ is a small positive number. This is because the terms $\left|\lambda v_{d} v_{u}-k x^{2}\right|^{2}$ and $A_{\lambda} \lambda v_{d} v_{u} x$, when $\lambda v_{d} v_{u}$ and $k$ are of the same sign, both "push" the VEV of the real component of the singlet away from the origin. Unfortunately, one finds that, for any choice of values of the input parameters, there are always particles with unacceptably small masses. To illustrate the situation, Table 5.1 presents numerical results for several representative points in different "corners" of the parameter space. The first two points represent the typical situation for the case of the low-energy GMSB, the next two are representative of the case of the high-energy GMSB, and the last one explores the extreme case of $\Lambda=10^{15}$ GeV. Points 1 and 3 have relatively large values of $k$, while points 2 and 4 have $k \ll 1$. Notice that in the table a similar limiting case for $\lambda$ was not considered. This is not a coincidence. It turns out that, for $\lambda \lesssim 0.2$, the dominant term in the potential is $V_{D}$, and $\tan \beta$ is forced to values very close to one. In this case, in order to correctly reproduce the top quark mass, one is forced to choose $h_{t}$ at the weak scale such that $h_{t}$ hits the Landau pole below the GUT scale. Only the cases where the couplings in the superpotential remain perturbative up to the 
GUT scale were considered. However, no such assumption is made in the analysis in the next subsection.

It can be seen that, in all the cases presented in Table 5.1, there are particles with unacceptably small masses. The result for the low-energy GMSB is not new and has been known for several years [111]. On the other hand, the situation with a high messenger scale had not been quantitatively studied in the literature to date. One expected feature that is indeed seen in points 3 and 5 is the increase of the pseudoscalar Higgs boson mass with $\Lambda$. This happens because the magnitude of $A_{\lambda}$, generated by running the RG equations, increases with the messenger scale, and it is $A_{\lambda}$ that breaks the $U(1)$ symmetry of the potential, as discussed before. Another result that could have been anticipated is the smallness of the mass of the light pseudoscalar Higgs when $k \ll 1$ (points 2 and 4 ). This is due to the PecceiQuinn symmetry, which is restored in this limit. What is surprising is that raising the messenger scale by 10 orders of magnitude does not bring any other significant changes to the particle spectrum. The masses of the gluino, right-handed selectron, and scalar Higgs boson still remain small.

\subsubsection{Analytical Considerations}

This subsection contains a rather simple semi-analytical argument which explains why there can be no phenomenologically acceptable solution to the NMSSM with the GMSB. It is shown that if one assumes that such a solution exists, one arrives at a contradiction. Some of the features of the numerical solutions presented 
Table 5.1: The numerically determined NMSSM parameters for five sample points in the parameter space. Here $m_{h_{i}}$ and $m_{A_{i}}$ refer to the eigenvalues of the scalar and pseudoscalar Higgs mass matrices respectively, and $m_{\tilde{e}}$ denotes the mass of the right-handed selectron. The values of $\lambda, k$, and $h_{t}$ are given at the weak scale. All the other quantities have been defined earlier in the text.

\begin{tabular}{|c|c|c|c|c|c|c|}
\hline & \multicolumn{7}{|c|}{ Input Parameters } \\
\hline point & $\Lambda(\mathrm{GeV})$ & $\lambda$ & $k$ & $\mathrm{~B}(\mathrm{TeV})$ & $n$ & $h_{t}$ \\
\hline 1 & $5 \times 10^{4}$ & 0.25 & 0.1 & 6.4 & 1 & 1.12 \\
\hline 2 & $10^{5}$ & 0.28 & $3 \times 10^{-4}$ & 3.6 & 3 & 1.08 \\
\hline 3 & $10^{12}$ & 0.32 & 0.3 & 0.99 & 10 & 1.07 \\
\hline 4 & $10^{12}$ & 0.25 & $3 \times 10^{-4}$ & 6.0 & 1 & 1.11 \\
\hline 5 & $10^{15}$ & 0.28 & 0.3 & 6.9 & 1 & 1.07 \\
\hline
\end{tabular}

\begin{tabular}{|c|c|c|c|c|c|}
\hline & \multicolumn{5}{|c|}{ Soft SUSY-breaking Parameters at the Weak Scale } \\
\hline point & $m_{H_{u}}^{2}\left(\mathrm{GeV}^{2}\right)$ & $m_{H_{d}}^{2}\left(\mathrm{GeV}^{2}\right)$ & $m_{N}^{2}\left(\mathrm{GeV}^{2}\right)$ & $A_{\lambda}(\mathrm{GeV})$ & $A_{k}(\mathrm{GeV})$ \\
\hline 1 & $-2.4 \times 10^{3}$ & $5.3 \times 10^{2}$ & 4.6 & -1.5 & $-4.0 \times 10^{-3}$ \\
\hline 2 & $-2.8 \times 10^{3}$ & $5.7 \times 10^{2}$ & 6.8 & -2.6 & $-6.2 \times 10^{-3}$ \\
\hline 3 & $-3.1 \times 10^{3}$ & $4.8 \times 10^{2}$ & 29 & -11.4 & -0.15 \\
\hline 4 & $-2.5 \times 10^{3}$ & $6.8 \times 10^{2}$ & 12 & -8.0 & -0.11 \\
\hline 5 & $-2.9 \times 10^{3}$ & $1.0 \times 10^{3}$ & -8.1 & -9.4 & $-6.0 \times 10^{-3}$ \\
\hline
\end{tabular}

\begin{tabular}{|c|c|c|c|c|c|c|}
\hline & \multicolumn{2}{|c|}{ Field VEVs } & \multicolumn{4}{c|}{ Particle Masses } \\
\hline point & $\tan \beta$ & $x(\mathrm{GeV})$ & $M_{3}(\mathrm{GeV})$ & $m_{\tilde{e}}(\mathrm{GeV})$ & $m_{h_{i}}(\mathrm{GeV})$ & $m_{A_{i}}(\mathrm{GeV})$ \\
\hline 1 & 1.59 & -3.7 & 61 & 32 & $85,39,35$ & $51,1.8$ \\
\hline 2 & 1.84 & -3.7 & 103 & 35 & $87,48,38$ & $48,0.2$ \\
\hline 3 & 1.97 & -40 & 94 & 36 & $87,53,28$ & 76,25 \\
\hline 4 & 1.63 & -14 & 57 & 34 & $85,43,37$ & $44,0.5$ \\
\hline 5 & 1.88 & -49 & 66 & 40 & $88,50,27$ & 71,24 \\
\hline
\end{tabular}


in the previous subsection are also explained.

Suppose that for some point in the parameter space an acceptable solution exists. The problem to be addressed is the smallness of the selectron, gluino, and Higgs masses. For simplicity the analysis is based on the right-handed selectron mass constraint. The magnitude of $m_{\tilde{e}}$ is directly proportional to the size of the $B$-parameter. In our numerical procedure the value of $B$ is chosen in such a way that $v=174 \mathrm{GeV}$. A typical value of $B$ obtained in this way yields a very small selectron mass $\left(m_{\tilde{e}} \sim 35 \mathrm{GeV}\right)$, gluino mass $\left(M_{3} \lesssim 100 \mathrm{GeV}\right)$, and soft SUSYbreaking masses for the Higgs bosons $\left(m_{H_{u}}^{2} \sim-3000 \mathrm{GeV}^{2}, m_{H_{d}}^{2} \sim 500 \mathrm{GeV}^{2}\right)$.

It is, therefore, obvious that the only chance of obtaining an acceptable value for the selectron mass is to raise $B$, by a factor of three or more, and try to arrange the other parameters in such a way that $\sqrt{v_{d}^{2}+v_{u}^{2}}$ remains $174 \mathrm{GeV}$. Since $B$ feeds into all soft SUSY-breaking masses, their absolute values will also increase. For example, imposing $m_{\tilde{e}}>80 \mathrm{GeV}$ forces $m_{H_{u}}^{2}<-(215 \mathrm{GeV})^{2}$ for a messenger scale of $10^{16} \mathrm{GeV}$. For different messenger scales the bound becomes even more stringent, as shown in Fig 5.2(a).

To determine the consequences of raising the soft SUSY-breaking masses, the Higgs potential (Eq. (5.4.5)) will be analyzed. The extremization conditions at tree level are

$$
\begin{aligned}
\frac{\partial V_{\text {neutral }}^{\text {tree }}}{\partial v_{d}}= & 2\left(\lambda v_{d} v_{u}-k x^{2}\right) \lambda v_{u}+2 \lambda^{2} x^{2} v_{d}+2 m_{H_{d}}^{2} v_{d}-2 A_{\lambda} \lambda v_{u} x+ \\
& +\frac{g^{\prime 2}+g_{2}^{2}}{4} 2 v_{d}\left(v_{d}^{2}-v_{u}^{2}\right)
\end{aligned}
$$



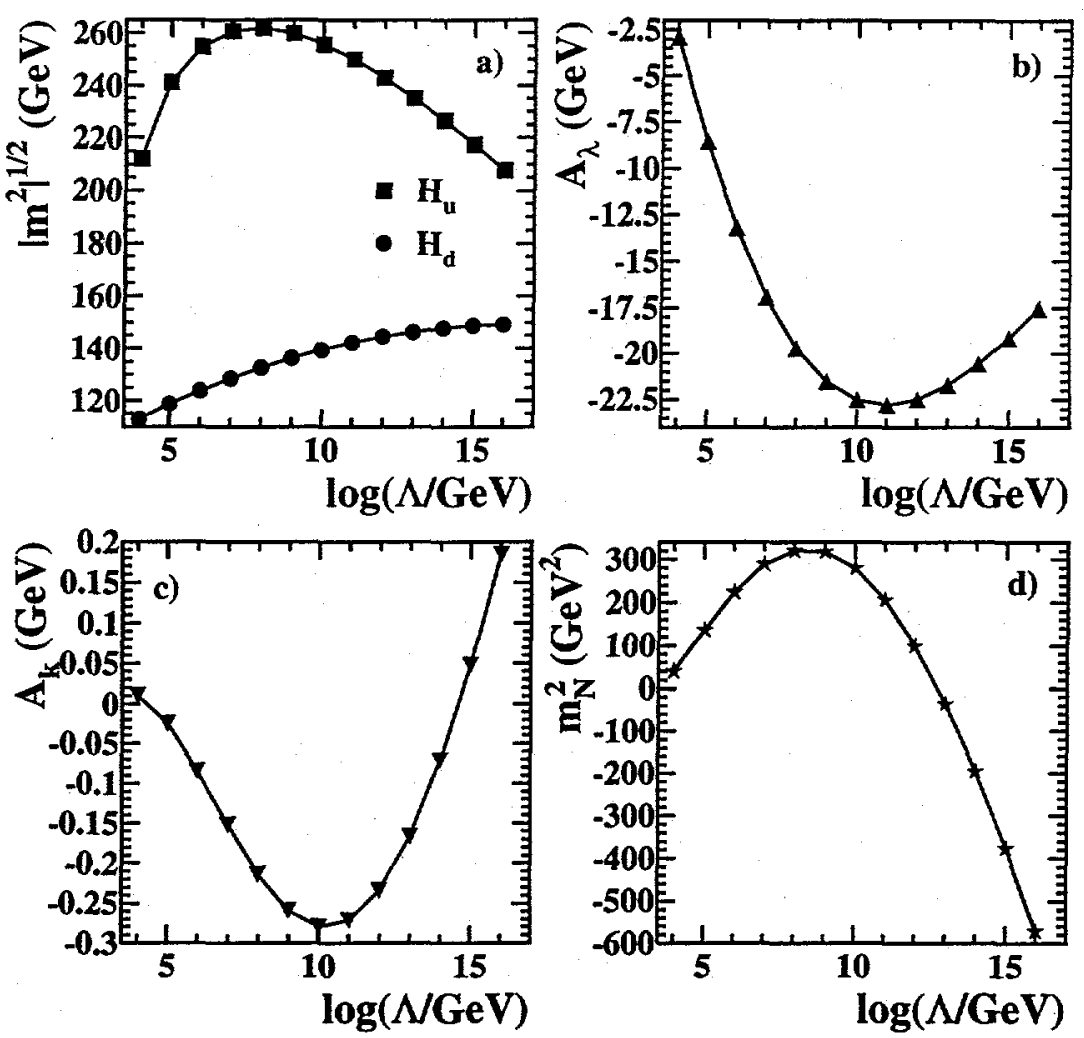

Figure 5.2: (a) Lower bounds on $\left|m_{H_{u}}^{2}\right|^{1 / 2}$ and $\left|m_{H_{d}}^{2}\right|^{1 / 2}$ as a function of the messenger scale $\Lambda$ from the selectron mass constraint $m_{\tilde{e}}>80 \mathrm{GeV}$. Here $n=1$, $h_{t}=1.07, k=0.3$ and $\lambda=0.29$ at the weak scale. These bounds do not change for different values of $k$ or $\lambda$. The other plots show typical values of (b) $A_{\lambda}$, (c) $A_{k}$, and (d) $m_{N}^{2}$, for the same choice of parameters that yielded (a). The values of these parameters do not change significantly for different values of $k$ or $\lambda$. 


$$
\begin{aligned}
\frac{\partial V_{n e u t r a l}^{\text {tree }}}{\partial v_{u}}= & 2\left(\lambda v_{d} v_{u}-k x^{2}\right) \lambda v_{d}+2 \lambda^{2} x^{2} v_{u}+2 m_{H_{u}}^{2} v_{u}-2 A_{\lambda} \lambda v_{d} x+ \\
& +\frac{g^{2}+g_{2}^{2}}{4} 2 v_{u}\left(v_{u}^{2}-v_{d}^{2}\right) \\
\frac{\partial V_{\text {neutral }}^{\text {tree }}=}{\partial x}= & 2\left(\lambda v_{d} v_{u}-k x^{2}\right)(-2 k x)+2 x \lambda^{2}\left(v_{d}^{2}+v_{u}^{2}\right)+2 m_{N}^{2} x- \\
& -2 A_{\lambda} \lambda v_{d} v_{u}-2 k A_{k} x^{2}
\end{aligned}
$$

The first two equations (Eqs. (5.4.8) and (5.4.9)) closely resemble the corresponding ones in the MSSM case. In fact, the only difference in the NMSSM is the presence of the first term on the right-hand side of Eq. (5.4.8) and Eq. (5.4.9). This term originates from $\left|\frac{\partial W}{\partial x}\right|^{2}=\left|\lambda v_{d} v_{u}-k x^{2}\right|^{2}$ and is, therefore, absent in the MSSM. Dividing Eq. (5.4.8) by $v_{u}$, Eq. (5.4.9) by $v_{d}$, and subtracting the two expressions, this term can be canceled. As a result, one obtains

$$
\begin{aligned}
\lambda^{2} x^{2} & =-\frac{M_{Z}^{2}}{2}-\frac{1}{2}\left(m_{H_{d}}^{2}+m_{H_{u}}^{2}\right)-\frac{1}{2} \frac{m_{H_{d}}^{2}-m_{H_{u}}^{2}}{\cos 2 \beta} \\
& =-\frac{M_{Z}^{2}}{2}+\frac{m_{H_{d}}^{2}-m_{H_{u}}^{2} \tan ^{2} \beta}{\tan ^{2} \beta-1} .
\end{aligned}
$$

Note that this equation is identical to Eq. (5.3.5) with $\mu \equiv \lambda x$. To obtain the NMSSM analog of Eq. (5.3.6) one divides Eq. (5.4.8) by $v_{d}$, Eq. (5.4.9) by $v_{u}$ and add the two. Solving for $A_{\lambda} \lambda x\left(A_{\lambda} \lambda x \equiv m_{3}^{2}\right)$ one finds:

$$
A_{\lambda} \lambda x=\left(m_{H_{d}}^{2}+m_{H_{u}}^{2}+2 \lambda^{2} x^{2}\right) \frac{\sin 2 \beta}{2}+\lambda\left(\lambda v_{d} v_{u}-k x^{2}\right)
$$

Eqs. (5.3.6) and (5.4.12) differ only by the contribution from $\left|\frac{\partial W}{\partial x}\right|^{2}$.

Eq. (5.4.11) states that the value of the effective $\mu$-parameter generated in this model is subject to a rather stringent bound: $\lambda^{2} x^{2}>-m_{H_{u}}^{2}-M_{Z}^{2} / 2$, which, if one imposes $m_{H_{u}}^{2}<-(212 \mathrm{GeV})^{2}$, translates into $\lambda x>200 \mathrm{GeV}$. Notice that the 
origin of this bound is the same as of the bound on the size of the $\mu$-parameter derived in Sec. 5.3, since the condition given by Eq. (5.4.11) is the same in both cases. In the present case, however, the bound is stronger because $\tan \beta$ is no longer a free parameter but is determined by minimizing the Higgs potential.

So far only the first two extremization conditions were looked at. The attention is now turned to Eq. (5.4.10). Solving for $x^{2}$ in Eq. (5.4.11), one can rewrite Eq. (5.4.10) as $2 \frac{k^{2}}{\lambda^{2}}\left(\frac{m_{H_{d}}^{2}-m_{H_{u}}^{2} \tan ^{2} \beta}{\tan ^{2} \beta-1}-\frac{M_{Z}^{2}}{2}\right)=\lambda v^{2}(k \sin 2 \beta-\lambda)-m_{N}^{2}+A_{\lambda} \lambda v^{2} \frac{\sin 2 \beta}{2 x}+k A_{k} x$.

While it was shown that phenomenology requires the expression in parenthesis on the left-hand side to be larger than $(200 \mathrm{GeV})^{2}$, the terms on the right-hand side are all much smaller, because $m_{N}^{2}, A_{\lambda}$, and $A_{k}$ are zero at the messenger scale and the effects of the RG running are relatively small (see Fig. 5.2). This means that the above equation can never be satisfied unless $k \ll \lambda$.

An immediate consequence of the $k \rightarrow 0$ limit is that the mass of the lightest pseudoscalar Higgs goes to zero, as it becomes a Nambu-Goldstone boson. (It is for this reason that $k$ was introduced in the first place.) Furthermore in the limit of large $\mu$ and small $k$ the determinant of the scalar Higgs mass-squared matrix becomes negative, which means that the extremum point given by Eqs. (5.4.8-5.4.10) ceases to be a minimum. To show this it is necessary to first derive a relationship between $k$ and $\sin 2 \beta$. Such a relationship can be derived from Eq. (5.4.11) and Eq. (5.4.12). Neglecting $M_{Z}^{2}, A_{\lambda} \lambda x$, and $\lambda^{2} v^{2}$ in comparison to $m_{H_{d}}^{2}$ and $m_{H_{u}}^{2}$, one 
finds that

$$
\sin 2 \beta=\frac{k}{\lambda} \frac{1}{1+r^{2}\left(\frac{k}{\lambda}\right)^{2}}\left(1+r \sqrt{1+\left(r^{2}-1\right)\left(\frac{k}{\lambda}\right)^{2}}\right)
$$

Here $r \equiv-\left(m_{H_{d}}^{2}+m_{H_{u}}^{2}\right) /\left(m_{H_{d}}^{2}-m_{H_{u}}^{2}\right){ }^{* *}$ For $k \ll \lambda$, Eq. (5.4.14) reduces to

$$
\sin 2 \beta \approx 2 \frac{k}{\lambda}\left[\frac{-m_{H_{u}}^{2}}{m_{H_{d}}^{2}-m_{H_{u}}^{2}}\right]
$$

Equipped with the last result, one can consider the determinant of the scalar Higgs mass-squared matrix. The full expression for it is given in Eq. G.1; here only the leading terms need to be identified. One is interested in the case $\mu>\lambda v$, and, as was argued before, the soft trilinear couplings $A_{\lambda}$ and $A_{k}$ can be neglected. For this reason, the dominant terms will be the ones containing the highest power of $\mu$ :

$$
\operatorname{det} \mathcal{M}_{s c a l a r}^{2} \simeq \frac{2 v^{2} \mu^{4}}{\lambda^{3} \sin (2 \beta)}\left(-4 k \lambda^{4}+k^{3} \bar{g}^{2}+k^{3} \cos (4 \beta) \bar{g}^{2}+8 k^{2} \lambda^{3} \sin (2 \beta)\right)
$$

That these are indeed the largest terms was checked numerically.

Taking into account the fact that $k$ and $\sin 2 \beta$ are proportional to each other for small $k$, one can easily see that, in the limit $k \rightarrow 0$, the first term dominates and the determinant is negative. ${ }^{\dagger \dagger}$

This completes the argument, and one is now able to state that there can be no phenomenologically viable solution in the context of the NMSSM. One could have

${ }^{* *}$ In deriving Eq. (5.4.14) it was necessary to assume that $\sin 2 \beta>k / \lambda$. This translates into two requirements: $r>0$ and $k / \lambda<1$. One concludes that for large soft SUSY-breaking Higgs masses-squared it is necessary to have $\lambda>k$.

${ }^{\dagger}$ Because $\sin 2 \beta \propto k / \lambda$ there is no ambiguity with sign redefinitions of $\lambda$ or $k$ in Eq. (5.4.16). 
also based the argument on the gluino mass bound. The experimental constraint $M_{3}>190 \mathrm{GeV}$ translates into the requirement $m_{H_{u}}^{2}<-(212 \mathrm{GeV})^{2}$ (assuming $n=1$ ), and the rest of the argument follows unchanged. Notice, however, that the bound on $m_{H_{u}}^{2}$ weakens if the number of messenger fields is taken to be very large.

Now the issue of interpreting the numerical results of the previous subsection is addressed. It is important to understand, for instance, why the values of the singlet VEV $x$ in Table 5.1 are always smaller than the VEVs of the Higgs doublets and, furthermore, why $x$ is only several $\mathrm{GeV}$ for a low messenger scale.

The answer comes from considering the extremization condition for $x$ :

$$
2 k^{2} x^{3}+\lambda(\lambda-k \sin (2 \beta)) v^{2} x-\frac{v^{2} \lambda \sin (2 \beta) A_{\lambda}}{2} \simeq 0
$$

where the terms $m_{N}^{2}$ and $k A_{k} x$ were omitted $\left(\left|m_{N}^{2}\right| \ll \lambda^{2} v^{2}\right.$ for all the points in the table). For most of the parameter space the cubic term in $x$ can also be neglected, giving

$$
x \simeq \frac{A_{\lambda}}{\lambda} \frac{\sin (2 \beta)}{2\left(1-\frac{k}{\lambda} \sin (2 \beta)\right)} .
$$

Thus the smallness of $x$ is related to the fact that $A_{\lambda}$ is small. The above approximation holds as long as

$$
A_{\lambda}^{2}<\lambda^{2} v^{2} \frac{\lambda^{2}}{k^{2}} \frac{2\left(1-\frac{k}{\lambda} \sin 2 \beta\right)^{3}}{\sin ^{2} 2 \beta}
$$

which is not satisfied only for point 5 in Table 5.1. For point 5 the value of $x$ can 
be approximated by

$$
x \simeq\left(A_{\lambda} v^{2} \frac{\lambda \sin 2 \beta}{4 k^{2}}\right)^{1 / 3} .
$$

Again $x<v$ and therefore $\lambda x \ll 175 \mathrm{GeV}$.

Knowing that $x$ is small in this model one can derive another interesting relation. Neglecting all the terms containing $x$ in Eq. (5.4.12), one obtains:

$$
\lambda^{2} v^{2} \simeq-\left(m_{H_{d}}^{2}+m_{H_{u}}^{2}\right) .
$$

This explains why the values of the soft SUSY-breaking masses for the Higgs bosons are so similar for very different values of the messenger scale.

Finally, one can say a few words about the scalar Higgs boson masses. In the limit of small $x$ (and hence small $\mu$ ), the dominant term in the determinant of the scalar Higgs mass-squared matrix (see Appendix G) is

$$
\operatorname{det} \mathcal{M}_{\text {scalar }}^{2} \simeq \frac{3 A_{\lambda} v^{6} \lambda^{4} \bar{g}^{2}}{32 \mu \sin (2 \beta)}
$$

Taking into account the fact that, for small $x, \mu \equiv \lambda x \sim A_{\lambda}$ (see Eq. (5.4.18)), the equation above gives:

$$
m_{h_{1}}^{2} m_{h_{2}}^{2} m_{h_{3}}^{2} \sim \frac{3 v^{6} \lambda^{4} \bar{g}^{2}}{32 \sin (2 \beta)}
$$

This explains why changes in the messenger scale have almost no effect on the product of the scalar Higgs boson masses (see Table 5.1), as long as $\lambda$ is unchanged. For $\sin (2 \beta) \sim 0.8-0.9$, which is what one typically finds in this case, Eq. (5.4.23) gives a "geometrical average" value of the scalar Higgs boson mass of only about 
$50 \mathrm{GeV}$. This means that, as long as $x$ is small, the model necessarily yields phenomenologically unacceptable Higgs boson masses.

\subsection{Possible Modifications to the NMSSM}

In this section the expressions derived in Sec. 5.4 are reexamined and one attempts to modify the NMSSM to make it phenomenologically viable. Several possibilities are studied and the problems that arise are identified and discussed. Overall, none of the possibilities are found to be entirely satisfactory.

\subsubsection{Extra Vector-like Quarks}

One wants to modify the NMSSM in a way that allows one to avoid the conclusions of Sec. 5.4. Recall that the crucial step in the analysis there was the observation that Eq. (5.4.13) could not be satisfied: the left-hand side was always greater than the right-hand side. To obtain a consistent solution one has to somehow make both sides equal. One possibility is to make $m_{N}^{2}$ of the same order of magnitude (and sign) as $m_{H_{u}}^{2}$. That could be accomplished by coupling the singlet to some new fields and arranging the parameters in such a way that the SUSYbreaking mass-squared of the singlet is driven sufficiently negative. This idea was first proposed by Dine and Nelson in Ref. [111], who introduced new color-triplet fields $q^{\prime}$ and $\bar{q}^{\prime}$ and coupled them to $N$. The corresponding superpotential is

$$
W=h_{u} Q H_{u} u^{c}+h_{d} Q H_{d} d^{c}+h_{e} L H_{d} e^{c}+\lambda N H_{d} H_{u}-\frac{1}{3} k N^{3}+\lambda_{q} N q^{\prime} \bar{q}^{\prime}
$$


According to Eq. (5.2.6), the scalar components of $q^{\prime}$ and $\bar{q}^{\prime}$ acquire large SUSYbreaking masses, which can drive $m_{N}^{2}$ sufficiently negative.

Agashe and Graesser in Ref. [137] did a quantitative study of this scenario for the case of the low-energy GMSB. They showed that it is indeed possible to generate a large negative $m_{N}^{2}$, in the range $-(150 \mathrm{GeV})^{2}$ to $-(200 \mathrm{GeV})^{2}$, and further demonstrated that, with $m_{N}^{2}$ of this magnitude, one can choose the input parameters in such a way that $v=174 \mathrm{GeV}$ and all experimental constraints are evaded. They also pointed out that in this scenario the input parameters need to be fine-tuned in order to reproduce the above value of $v$. In what follows, a set of input parameters that yields an acceptable particle spectrum is given, and then one proceeds to analyze the sensitivity of the Higgs boson VEVs to the NMSSM coupling constants. The origin of this sensitivity is described and also extend the analysis to the case of the high-energy GMSB.

As an example of an allowed solution, consider the case of the low-energy GMSB with $B=50 \mathrm{TeV}, n=1$, and $\Lambda=100 \mathrm{TeV}$. For $m_{N}^{2}=-(190 \mathrm{GeV})^{2}$, to correctly reproduce $M_{Z}$ and $m_{t}$ one takes $h_{t}=0.99, k=-0.045$ and $\lambda=0.11$ at the weak scale. It is found that $\tan \beta$ equals -2.9 for this point. Because the magnitude of the product $B n$ is now quite large, the masses of the gluino and right--handed selectron are safe: $M_{3}=477 \mathrm{GeV}, m_{\tilde{e}}=93 \mathrm{GeV}$. The vacuum expectation value of the singlet is also large, $x=2.97 \mathrm{TeV}$, which, as was argued earlier, is required by Eq. (5.4.11). The eigenvalues of the scalar Higgs mass matrix are 404,270 , and $90 \mathrm{GeV}$, and those of the pseudoscalar Higgs mass matrix are 
400 and $6.7 \mathrm{GeV}$. The last number appears alarmingly small at first sight but, as shown in Ref. [137], has not been excluded. The reason is that the corresponding eigenstate $a$ is almost a pure singlet:

$$
|a\rangle=0.031\left|H_{d}\right\rangle-0.011\left|H_{u}\right\rangle-0.999|N\rangle
$$

The quantitative criterion given in Ref. [137], based on the constraint from the $\Upsilon \rightarrow a \gamma$ decay, is

$$
\frac{\sin 2 \beta \tan \beta}{\sqrt{\left(\frac{x}{250 \mathrm{GeV}}\right)^{2}+\sin ^{2} 2 \beta}}<0.43,
$$

and for the parameter set above the left-hand side equals 0.15 .

In this scheme it is, therefore, possible to find a point in the parameter space which leads to a phenomenologically viable solution. Unfortunately, as was already mentioned, this solution is very sensitive to the choice of the superpotential coupling constants $\lambda$ and $k$. In the remainder of this subsection, this issue is discussed in detail.

The values of the parameters for the set just described had to be chosen in such a way that the top quark and $Z$-boson masses were fixed at their known experimental values. It is interesting to investigate what values of $M_{Z}$ would be predicted for a generic choice of the parameters. Fig. 5.3 depicts the magnitude of the quantity $v \equiv \sqrt{v_{d}^{2}+v_{u}^{2}}$ as a function of $\lambda$ and $k$. The figure shows that small changes in both $\lambda$ and $k$ lead to large changes in $v$. This is very similar to the situation in the MSSM which was considered in Sec. 5.3. There it was shown that the value of the $\mu$-parameter had to be chosen very carefully in order to yield 


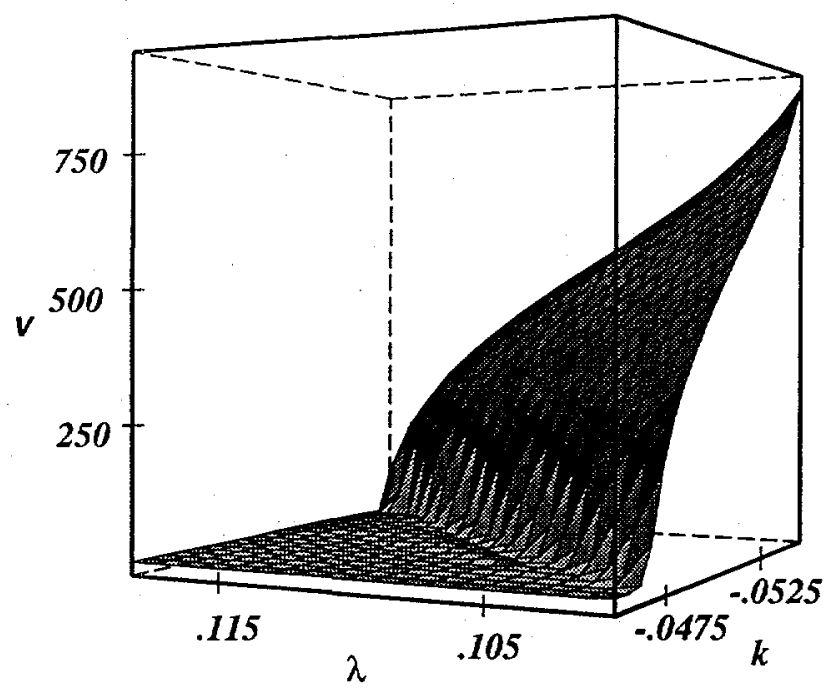

Figure 5.3: The value of $v \equiv \sqrt{v_{d}^{2}+v_{u}^{2}}$ as a function of $\lambda$ and $k$. The inputs are $n=1, m_{N}^{2}=-(190 \mathrm{GeV})^{2}, B=50 \mathrm{TeV}, \Lambda=100 \mathrm{TeV}, h_{t}=0.99$.

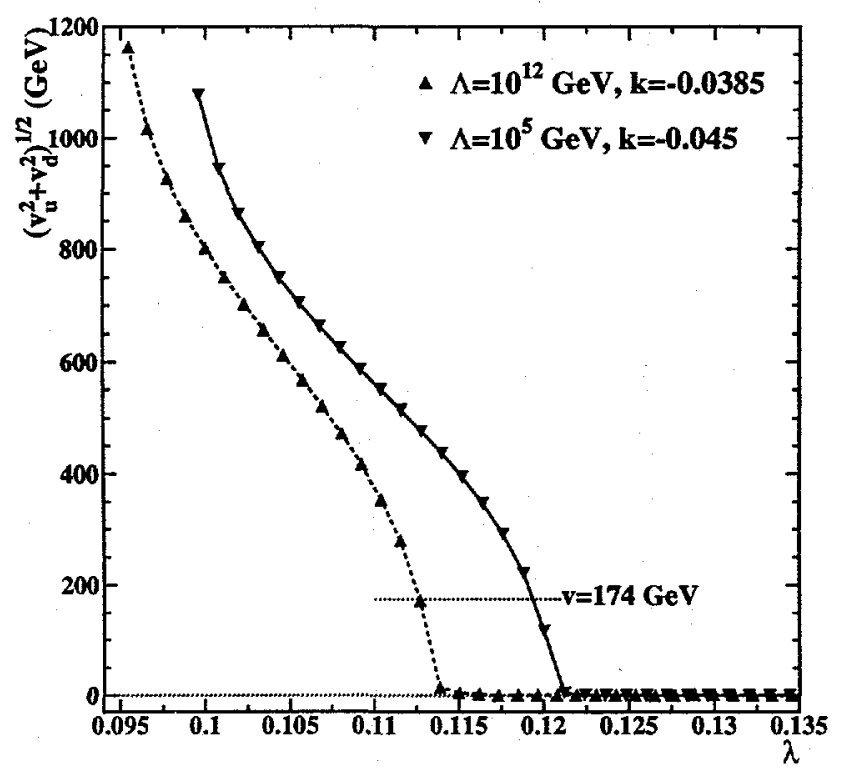

Figure 5.4: The dependence of $v$ on the value of $\lambda$ for the high- and low-energy GMSB. The other input parameters are the same as in Fig. 5.3. 
the correct value of $v$. In the present case, the points in the parameter space that correspond to values of $v$ around $174 \mathrm{GeV}$ lie in a very thin band on the $\lambda-k$ plane. Also notice that, for this range of $\lambda$ and $k$, the slope is the steepest. (See Appendix $\mathrm{H}$ for comments on this point.)

It is possible to perform the same type of analysis for a higher messenger scale. The same problem is found in that case as well. Fig. 5.4 depicts the dependence of $v$ on $\lambda$ for fixed values of $k$. For comparison, the curve for $\Lambda=10^{12} \mathrm{GeV}$ is plotted next to the curve for $\Lambda=100 \mathrm{TeV}$. From the slopes of these curves one can determine the degree of sensitivity with respect to $\lambda$, using the definition in Sec. 5.3. The degree of sensitivity, given by $\mathrm{d}(\log v) / \mathrm{d}(\log \lambda)$, is $2 \%$ for the lowenergy curve and $1 \%$ for the high-energy curve. The numerical results presented here agree with those in Ref. [137] for the low-energy GMSB, if the same inputs parameters are used.

In order to understand this behavior, one should once again turn to the extremization conditions Eqs. (5.4.8-5.4.10). First, some qualitative observations are presented. Recall that phenomenology requires $|x|$ to be rather large (of the order $\left.\sqrt{\left|m_{H_{u}}^{2}\right|} / \lambda \gtrsim 1 \mathrm{TeV}\right)$, while $v$ has to remain "small" $(v=174 \mathrm{GeV})$ to correctly reproduce $M_{Z}$. As a result, the terms containing high powers of $x$ and the terms containing $m_{i}^{2}\left(i=H_{d}, H_{u}, N\right)$ dominate, while the terms with $v_{u}$ and $v_{d}$ are not fixed, and have to absorb the residual difference between the dominant terms. Therefore, small percentile changes in the dominant terms can result in large percentile changes in the Higgs boson VEVs. This is to be contrasted with 
the situation in the previous section, where $\lambda^{2} v^{2}$ was tied to the value of the sum $m_{H_{d}}^{2}+m_{H_{u}}^{2}$ (see Eq. (5.4.21)).

Next, the main source of this sensitivity is identified. First consider the dependence of $v$ on $\lambda$ for fixed $B, k$, and $h_{t}$. One can use Eq. (5.4.11) to solve for $v^{2}$ and then isolate the largest contribution to $\partial v / \partial \lambda$.

$$
\begin{aligned}
\frac{\partial v^{2}}{\partial \lambda}=\frac{4}{\bar{g}^{2}}\left[-2 \lambda x^{2}-2 \lambda^{2} x \frac{\partial x}{\partial \lambda}\right. & +\frac{\partial}{\partial \tan \beta}\left(\frac{m_{H_{d}}^{2}-m_{H_{u}}^{2} \tan ^{2} \beta}{\tan ^{2} \beta-1}\right) \frac{\partial \tan \beta}{\partial \lambda} \\
& \left.+\frac{1}{\tan ^{2} \beta-1} \frac{\partial m_{H_{d}}^{2}}{\partial \lambda}-\frac{\tan ^{2} \beta}{\tan ^{2} \beta-1} \frac{\partial m_{H_{d}}^{2}}{\partial \lambda}\right] .
\end{aligned}
$$

Using the data that led to Fig. 5.3, one may numerically evaluate the derivative around the point $\lambda=0.11, k=-0.045$. The following are the results of evaluating each of the terms on the right-hand side, respectively: $-1.4 \times 10^{7},-2.4 \times$ $10^{6},-3.9 \times 10^{6},-1.2 \times 10^{3}, 1.9 \times 10^{1}\left(\mathrm{GeV}^{2}\right)$. The largest term is the first one, the next two terms combined provide a $45 \%$ correction, and the derivatives of the soft SUSY-breaking masses can be completely neglected. In Appendix I it is shown how these numbers can be understood by studying the minimization conditions.

The fact that the dominant contribution to $\partial v / \partial \lambda$ comes from the first term in Eq. (5.5.4) has a very important implication. It means that the problerns of cancellation in the NMSSM and the MSSM are not merely similar, but have exactly the same origin. Indeed, Eq. (5.4.11) is the same as Eq. (5.3.5), and, because in the NMSSM $v$ depends on $\lambda$ mainly through the combination $\lambda x$, which plays the role of the $\mu$-term, the two models require roughly the same degree of cancellation. The degree of cancellation quoted in Sec. 5.3 for the MSSM is most conservatively 
$16 \%$, but this is so because one can choose $\tan \beta$ freely in the MSSM. On the other hand, $\tan \beta$ is determined by minimizing the potential for the NMSSM and cannot be chosen arbitrarily to ease the cancellation. For the value of $\tan \beta$ obtained in the NMSSM, the degree of cancellation is actually comparable (order a few percent) in the MSSM. The small difference between the two models is due to the dependence of $x$ and $\tan \beta$ on $\lambda$.

The $\lambda$ dependence of the Higgs boson VEV has been discussed, and now the $k$ dependence is studied. Fig. 5.3 shows that the points that yield $v=174 \mathrm{GeV}$ form an almost straight line on the $\lambda-k$ plane. It can be shown (see I) that in order to keep $v$ constant one has to change $k$ and $\lambda$ according to $\Delta k / k=\Delta \lambda / \lambda$. The sensitivity of $v$ to $k$ is, thus, related to the sensitivity of $v$ to $\lambda$, which, in turn, originates from the need to carefully choose the $\mu$-parameter in the MSSM as discussed in Sec. 5.3.

To summarize, it was shown that this model requires a very particular choice of parameters to yield the correct $Z$-boson mass. Furthermore, it was explained that the sensitivity of the $Z$-boson mass to the NMSSM couplings has the same origin as the sensitivity of the $Z$-boson mass to the value of the $\mu$-parameter in the MSSM. It is worthwhile to emphasize that the problem is present for both high and low messenger scales, simply because the bound on the $\mu$-parameter does not weaken as one raises the messenger scale. 


\subsubsection{Hypercharge $D$-term}

This subsection investigates what happens if the $D$-term contributions described at the end of Sec. 5.3 are included. First, the case of the NMSSM with no extra particles added is considered. One should try to determine if, by introducing the $D$-terms, it is possible to make $v$ smaller. If that happened, $v$ could be rescaled back by increasing $B$, and that would raise all masses in the model, as desired. It turns out that this is not the case. Upon adding the $D$-terms both $\tan \beta$ and $\langle x\rangle$ change, but $v_{d}^{2}+v_{u}^{2}$, curiously enough, remains virtually constant. This happens because, in the limit $x^{2} \ll v^{2}, v^{2}$ is constrained by Eq. (5.4.21), and the change $m_{H_{d}}^{2} \rightarrow m_{H_{d}}^{2}-\frac{1}{2} D_{Y}, m_{H_{u}}^{2} \rightarrow m_{H_{u}}^{2}+\frac{1}{2} D_{Y}$ preserves the quantity $m_{H_{d}}^{2}+m_{H_{u}}^{2}$.

The next question to ask is whether the $D$-terms can decrease the degree of cancellation for the case with $q^{\prime}$ and $\vec{q}^{\prime}$ added. The answer is again negative and the reason can be seen from Eq. (5.4.12). Recall that the degree of cancellation is controlled by the magnitude of $x^{2}$. As long as $A_{\lambda} \lambda x$ and $\lambda^{2} v_{d} v_{u}$ can be neglected compared to $m_{H_{d}}^{2}+m_{H_{u}}^{2}$, Eq. (5.4.12) yields

$$
x^{2} \simeq-\frac{\left(m_{H_{d}}^{2}+m_{H_{u}}^{2}\right)}{2 \lambda\left(\lambda-\frac{k}{\sin 2 \beta}\right)},
$$

and the relevant quantity is again $m_{H_{d}}^{2}+m_{H_{u}}^{2}$.

\subsubsection{Large Trilinear Couplings}

At last, the scenario proposed by Ciafaloni and Pomarol [136] is considered. They consider a modified version of the NMSSM, where $k=0, \lambda \ll 1$ and the value 
of $A_{\lambda}$ is large at the messenger scale. Their model also contains, in the potential at the weak-scale, a linear term in $N$ which is generated by tadpole diagrams and solves the problem of a light pseudoscalar. They find that the requirement of the positivity of the determinant of the scalar Higgs boson mass-squared matrix is very restrictive. Part of their analysis is repeated in order to determine if their choice of parameters could indeed lead to a phenomenologically viable electroweak symmetry breaking spectrum. Note that, as far as the following is concerned, their model is identical to the NMSSM.

The full expression for the determinant can be found in Eq. G.1. In the limit of $k \rightarrow 0$ and $\lambda \rightarrow 0$

$$
\operatorname{det} \mathcal{M}_{\text {scalar }}^{2} \simeq \frac{A_{\lambda}^{2} v^{2} M_{Z}^{2} \lambda^{2} \sin ^{2}(4 \beta)}{4(1+y)^{3}}\left[1+y-\frac{y^{2}}{\cos ^{2}(2 \beta)}\left(\frac{A_{\lambda}^{2}}{M_{Z}^{2}}+1\right)-\frac{y^{3}}{\cos ^{2}(2 \beta)}\right]
$$

where a variable $y \equiv \bar{g}^{2} m_{N}^{2} /\left(2 M_{W}^{2}\right)$ is introduced to conform to the notation used in Ref. [136]. From the extremization conditions for the potential, Eqs. (5.4.85.4.10), one can show that $\mu=A_{\lambda} \sin (2 \beta) /(2(1+y))$. There are two intervals of $y$ over which the determinant is greater than zero. One interval is where both the expression in the brackets and the denominator are positive. It is given approximately by the following bound on $|y|$ :

$$
|y|<\left|\cos 2 \beta\left(1+\frac{A_{\lambda}^{2}}{M_{Z}^{2}}\right)^{-1 / 2}\right| .
$$

The other interval, not mentioned in [136], is approximately $\left(-\left(\frac{A_{\lambda}^{2}}{M_{Z}^{2}}+1\right),-1\right)$, where both the denominator and the bracketed expression are negative. 
The first interval, for $A_{\lambda}>M_{Z}$, corresponds to rather small values of $m_{N}^{2}$ and

$$
\mu \simeq \frac{1}{2} A_{\lambda} \sin (2 \beta)
$$

Using this equation together with Eq. (5.4.12), one can derive the following result:

$$
A_{\lambda}^{2}\left(1-\frac{\sin ^{2}(2 \beta)}{2}\right) \simeq\left(m_{H_{u}}^{2}+m_{H_{d}}^{2}\right)
$$

The above equation is impossible to satisfy in models with the GMSB, because the combination $\left(m_{H_{u}}^{2}+m_{H_{d}}^{2}\right)$ is always negative at the weak scale for the messengerscale boundary conditions given by Eqs. (5.2.5) and (5.2.6). To satisfy Eq. (5.5.9), a drastic modification of the boundary conditions would be required.

Now, the second possibility is analyzed. It requires a relatively large negative value of the singlet soft SUSY-breaking mass-squared: $m_{N}^{2}<-2 /\left(\bar{g}^{2}\right) \times M_{W}^{2}=$ $-(132 \mathrm{GeV})^{2}$. This value is impossible to generate unless, as before, one introduces fields $q^{\prime}$ and $\vec{q}^{\prime}$ and couples them to $N$. Even with the introduction of these fields, if $k=0, \lambda \ll 1$, the extremization conditions cannot be simultaneously satisfied. This can be seen in the following way. For $k=0$ Eq. (5.4.13) takes on the form

$$
x=\frac{A_{\lambda} \lambda v^{2} \sin (2 \beta)}{m_{N}^{2}+\lambda^{2} v^{2}}
$$

which implies $x \rightarrow 0$ as $\lambda \rightarrow 0$. This is incompatible with Eq. (5.4.11), which requires that $x \rightarrow \infty$ as $\lambda \rightarrow 0$.

\subsection{Conclusion}


The issue of electroweak symmetry breaking in models with the gauge mediation of supersymmetry breaking (GMSB) was studied. First, a review of various proposals in the literature to generate the $\mu$-parameter of the MSSM with the same order of magnitude as the soft SUSY-breaking parameters such as squark, slepton, and gaugino masses was presented. It was determined that most of them require small parameters which are accidentally of the same magnitude as the loop factors, cancellation of the kinetic mixing terms at the level of $10^{-4}$, omission of interactions allowed by symmetries, or many new degrees of freedom not motivated otherwise.

Even if one could generate the $\mu$-parameter with the same order of magnitude as the soft SUSY-breaking parameters, it has to have particular values to reproduce $M_{Z}=91 \mathrm{GeV}$. This question was studied numerically and the following was found. The current experimental lower bounds on superparticle masses limit the overall scale of SUSY breaking from below, which in turn limits $m_{H_{u}}^{2}<0$ from above (i.e., $\left|m_{H_{u}}^{2}\right|$ from below). To reproduce $M_{Z}, \mu^{2}$ needs to cancel (too-negative) $m_{H_{u}}^{2}$ and is hence bounded from below. Therefore, there is some cancellation required between $\mu^{2}$ and $m_{H_{u}}^{2}$. Even with the most conservative set of parameters, it was determined that a cancellation of $16 \%$ is necessary. The situation is worse for most of the parameter space. This situation was contrasted to the minimal supergravity scenario, where the current experimental lower bounds on superparticle masses do not require a significant cancellation among parameters.

The simplest mechanism to generate the $\mu$-parameter would be the NMSSM, 
the minimal extension of the MSSM without dimensionful parameters in the superpotential. The NMSSM is known not to work with the low-energy GMSB, but there was hope that it might work with higher messenger scales. It was shown that this is unfortunately not the case. The current bounds on the superparticles masses are already strong enough to exclude the model completely. A semi-analytic discussion to clarify why the NMSSM fails was presented.

Various possible modifications to the NMSSM were also discussed, in particular whether they could lead to a viable electroweak symmetry breaking. The introduction of extra vector-like quarks coupled to the NMSSM singlet produces a large negative mass-squared for the singlet, and leads to a viable electroweak symmetry breaking. One needs to adjust the parameters to a few percent, which is comparable to the MSSM case for the same $\tan \beta$ range. A Fayet-Illiopoulos $D$-term for $U(1)_{Y}$ does not improve the situation.

The overall prospect of electroweak symmetry breaking with the GMSB remains unclear. It is hoped that the detailed investigation presented here prompts further studies on this issue. 


\section{Chapter 6}

\section{Conclusions}

In this dissertation, aspects of supersymmetric versions of the Standard Model of particle physics were studied. First, low energy supersymmetry (SUSY) was presented as one of the possible solutions to the gauge hierarchy problem.

In Chap. 2, the Minimal Supersymmetry Standard Model was introduced, including its many parameters and degrees of freedom. If SUSY is to stabilize the Higgs mass, it is necessary that a number of new "super-particles" be relatively light $(M \sim 100 \mathrm{GeV})$, and therefore accessible to the next generation of collider experiments. It was pointed out that already a significant portion of the MSSM parameter space is constrained, mainly due to the study of rare and forbidden processes and direct superparticle searches. It was also mentioned that SUSY searches are in general very model dependent.

The published OPAL 1991 and 1992 data on the QCD color factors [29] was reanalyzed in order to constrain possible additional contributions to four-jet events in $Z$ decays due to $q \bar{q} \tilde{g} \tilde{g}$ final states. It was determined that a light gluino with a mass below $1.5 \mathrm{GeV} / c^{2}$ is excluded at better than $90 \%$ confidence level. The result is insensitive to assumptions about what bound state it forms, the definition of its mass, and the gluino fragmentation provided that it does not decay inside 
the detectors. It is worthwhile to note that the currently available data set should be much more sensitive to a possible additional contribution from the light gluino. As a by-product of the analysis, the effect of finite bottom quark mass on $\mathrm{BZ}$ and NR distributions was discussed in detail, which turned out to be not negligible when extracting QCD color factors at current precisions.

In Chap. 3, the standard parameterizations of the MSSM soft SUSY breaking Lagrangian were described. These "simplifying" assumptions are necessary in order to render the MSSM phenomenologically viable and in order to allow one to make predictions of SUSY signals at colliders. One should, of course, try to avoid oversimplifying assumptions, which may disregard important phenomenological signatures for low energy SUSY. It was shown that the so-called "Minimal Supergravity Inspired" Supersymmetric Standard Model (VMSSM) is too restrictive as far as collider phenomenology is concerned. The addition of only one extra parameter to the VMSSM, the Fayet-Iliopoulos $D$-term for $U(1)_{Y}$, was proposed (this is referred to as the LSSM), and it was shown that it is capable of yielding a much more diverse phenomenology while still satisfying all experimental constraints. While the VMSSM almost always yields a B-ino-like $\tilde{\chi}_{1}^{0}$ LSP, the LMSSM also allows $\tilde{\nu}, \tilde{\tau}$ or Higgsino-like $\tilde{\chi}_{1}^{0}$ LSP. It was verified that for each one of these cases there are important phenomenological consequences, including new signatures for SUSY and the disappearance of other "standard" signatures.

In Chap. 4, the cosmology of models with the low-energy gauge mediation of SUSY breaking was studied. Initially, the lower bound on the gravitino mass 
was estimated, and it was shown that the bound conflicts with the cosmological constraint if the primordial gravitino is not diluted. This fact indicates a huge entropy production at a relatively low temperature, and the conventional baryogenesis scenario may not work well.

The Affleck-Dine baryogenesis was studied as an alternative and it was found that it works efficiently for an initial amplitude of the MSSM flat direction, $\left|\phi_{0}\right| \gtrsim 10^{13} \mathrm{GeV}$. It was also discussed that the decay of the flat direction may provide enough entropy to dilute the primordial gravitino for a relatively large initial amplitude of the flat direction, $\left|\phi_{0}\right| \gtrsim 10^{14-15} \mathrm{GeV}$. Therefore, the gravitino problem in model with the low-energy gauge mediation of SUSY breaking seems to be resolved if one assumes such a large initial amplitude.

The cosmological implication of the moduli fields in string theory was also discussed. Their masses are of the order of the gravitino mass, and their lifetime is much larger than the present age of the Universe in models with the low-energy gauge mediation of SUSY breaking. The mass density of the moduli field may overclose the Universe. To dilute the moduli fields, a very late inflation is needed. It was shown that the baryon asymmetry generated by Affleck-Dine baryogenesis can be large enough to survive such a late inflation for $\left|\phi_{0}\right| \gtrsim 10^{17} \mathrm{GeV}$, even if one assumes a huge entropy production to dilute the primordial moduli field below the critical density.

In Chap. 5, The issue of electroweak symmetry breaking in models with the gauge mediation of supersymmetry breaking (GMSB) was studied. First, a review 
of various proposals in the literature to generate the $\mu$-parameter of the MSSM with the same order of magnitude as the soft SUSY-breaking parameters such as squark, slepton, and gaugino masses was presented. It was determined that most of these proposals require small parameters which are accidentally of the same magnitude as the loop factors, cancellation of the kinetic mixing terms at the $10^{-4}$ level, omission of interactions allowed by symmetries, or many new degrees of freedom not motivated otherwise.

Even if one could generate the $\mu$-parameter with the same order of magnitude as the soft SUSY-breaking parameters, it has to have particular values to reproduce $M_{Z}=91 \mathrm{GeV}$. It was found that a cancellation between $\mu^{2}$ and (a too negative, due to experimental constraints on superparticle masses) $m_{H_{u}}^{2}$ is required. Even with the most conservative set of parameters, it was determined that a cancellation of $16 \%$ is necessary. The situation is worse for most of the parameter space. This situation is to be contrasted to the minimal supergravity scenario, where the current experimental lower bounds on superparticle masses do not require a significant cancellation among parameters.

The simplest mechanism to generate the $\mu$-parameter would be the NMSSM, the minimal extension of the MSSM without dimensionful parameters in the superpotential. The NMSSM is known not to work with the low-energy GMSB, but there was hope that it might work with higher messenger scales. It was shown that this is not the case. The current bounds on the superparticles masses are already strong enough to exclude the model completely. 
Various possible modifications to the NMSSM were also discussed, in particular whether they could lead to a viable electroweak symmetry breaking. The introduction of extra vector-like quarks coupled to the NMSSM singlet produces a large negative mass-squared for the singlet, and leads to a viable electroweak symmetry breaking. One needs to adjust the parameters to a few percent, which is comparable to the MSSM case for the same $\tan \beta$ range. A Fayet-Illiopoulos $D$-term for $U(1)_{Y}$ does not improve the situation. The overall prospect of electroweak symmetry breaking with the GMSB remains unclear, and more work is required.

In conclusion, SUSY is one of the most appealing solutions to the hierarchy problem. In order to test the hypothesis that Nature indeed is supersymmetric, phenomenological models of the low energy manifestations of SUSY are required. Such models have been developed over the past 20 years, and a great deal was/is being learned. Most important, independent of details of spectific models, it is clear to the community that the next generation of collider experiments will either find evidence for low energy SUSY or exclude it all together in the next 10 years. 


\section{Appendix A}

\section{Computing the Effective Potential}

The effective potential for the flat direction $\phi$ can be computed by the following usual procedure. One lets it have an expectation value, and calculates the vacuum energy in the presence of $\phi$ background. The vacuum energy is identically zero if one does not pick the effect of SUSY breaking. The lowest order contribution is from two-loop diagrams, where the standard model gauge multiplets couple to the vector-like messenger fields whose mass spectrum breaks SUSY. The gauge multiplets acquire masses because of the $\phi$ background and hence the result depends on $\phi$.

The mass spectrum of the messenger sector is $M$ for fermions, and $M_{ \pm}^{2}=M^{2} \pm$ $M B$ for scalars. One vector-like multiplet with unit $\mathrm{U}(1)$ charge is assumed, and the contribution from a $\mathrm{U}(1)$ gauge multiplet exchange is calculated. This $U(1)$ gauge group is the toy-model version of the standard model gauge groups. The flat

direction $\phi, \bar{\phi}$ also has \pm 1 charge under U(1), with $D$-flatness condition $\phi=\bar{\phi}$. The result can be easily generalized to arbitrary gauge groups and messenger multiplets. The $U(1)$ gauge coupling constant is referred to as $g$. The $U(1)$ gauge multiplet acquires a mass $m=2 g|\phi|$. The task is to calculate the vacuum energy as a function of $M, B, g$ and $m$. 


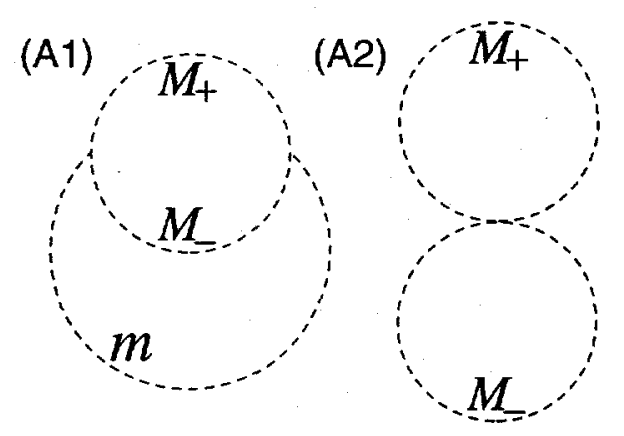

Figure A.1: Feynman diagrams which contribute to the vacuum energy in the background of the flat direction $\phi=\bar{\phi}$. The vertices are due to the $D$-term potential. The scalar field with mass $m=2 g\langle\phi\rangle$ is the scalar component of the massive gauge multiplet in the presence of the background $\phi$. The scalar fields with masses $M_{+}$and $M_{-}$are the messenger scalars.

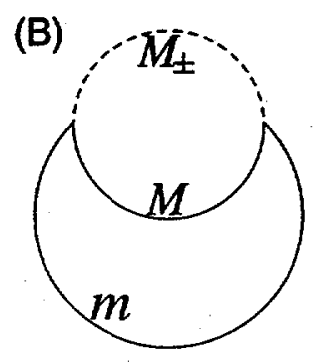

Figure A.2: A Feynman diagram with the gaugino of mass $m$, the messenger fermion of mass $M$, and the messenger scalars of mass $M_{ \pm}$. 

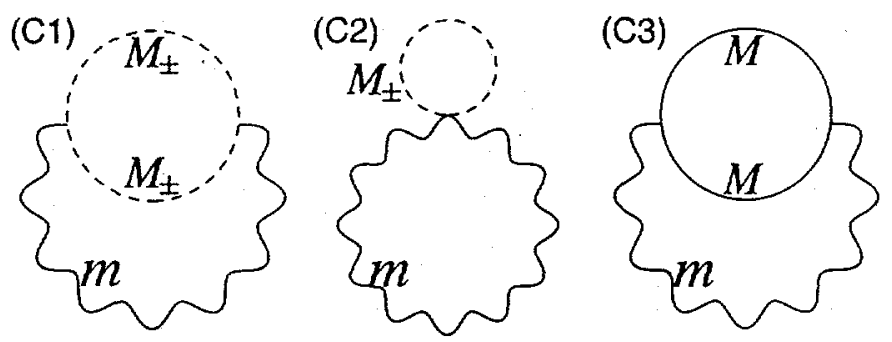

Figure A.3: Feynman diagrams with vacuum polarization due to (C1) messenger scalar loops, (C2) "seagull" diagram with messenger scalars, and (C3) messenger fermions.

The Feynman diagrams are shown in Figs. A.1-A.3. In all the calculations the amplitudes are expanded in terms of $M B / M^{2}$ and only the leading non-trivial terms of $O(M B)^{2}$ are kept. $M B / M^{2}<1$ is required in oreder to avoid a color- or charge-breaking vacuum, and this expansion is known to be a good approximation for the SUSY breaking mass squared for the flat direction unless $M B$ is very close to $M^{2}[105]$.

Start with diagrams (A1) and (A2) in Fig. A.1,

$$
\begin{aligned}
& (\mathrm{A} 1)=i g^{2} m^{2} \int \frac{d^{4} p}{(2 \pi)^{4}} \int \frac{d^{4} k}{(2 \pi)^{4}} \frac{1}{p^{2}-m^{2}}\left[\frac{1}{\left(k^{2}-M_{+}^{2}\right)\left((p+k)^{2}-M_{-}^{2}\right)}\right] \\
& (\mathrm{A} 2)=i g^{2} \int \frac{d^{4} p}{(2 \pi)^{4}} \int \frac{d^{4} k}{(2 \pi)^{4}}\left[\frac{1}{\left(k^{2}-M_{+}^{2}\right)\left(p^{2}-M_{-}^{2}\right)}\right]
\end{aligned}
$$

Since the sum of all diagrams vanishes in the supersymmetric limit $M B \rightarrow 0$, the corresponding amplitude in the supersymmetric limit is subtracted from each diagram. The diagrams (A1) and (A2) yield, after the subtraction:

$$
(\mathrm{A} 1)_{s}+(\mathrm{A} 2)_{s}=-i g^{2} \int_{0}^{1} d x \int \frac{d^{4} p}{(2 \pi)^{4}} \int \frac{d^{4} k}{(2 \pi)^{4}} \frac{p^{2}}{p^{2}-m^{2}}
$$




$$
\left[\frac{1}{\left(k^{2}+x(1-x) p^{2}-M^{2}+(1-2 x) M B\right)^{2}}-(M B \rightarrow 0)\right] .
$$

Here and hereafter, the subscript $s$ refers to the subtraction of amplitudes in the supersymmetric limit.

The integrand is expanded in powers of $M B / M^{2}$. The linear terms in $M B$ vanish upon the $x$ integration, and one is left with the following expression, to $O\left((M B)^{2}\right)$ in the integrand,

$$
\begin{aligned}
(\mathrm{A} 1)_{s}+(\mathrm{A} 2)_{s}= & i g^{2} \int_{0}^{1} d x \int \frac{d^{4} p}{(2 \pi)^{4}} \int \frac{d^{4} k}{(2 \pi)^{4}} \frac{p^{2}}{p^{2}-m^{2}} \frac{(4-1)(1-2 x)^{2}(M B)^{2}}{\left(k^{2}+x(1-x) p^{2}-M^{2}\right)^{4}} \\
& +O\left(B^{4}\right)
\end{aligned}
$$

The same strategy as above is followed to compute the contribution from the diagram (B) in Fig. A.2 containing the messenger fermions,

$$
\begin{aligned}
(\mathrm{B})_{s}= & -i(\sqrt{2} g)^{2} \int \frac{d^{4} p}{(2 \pi)^{4}} \int \frac{d^{4} k}{(2 \pi)^{4}} \frac{2(k \cdot p)}{\left(p^{2}-m^{2}\right)\left(k^{2}-M^{2}\right)} \\
& \left(\frac{1}{(p+k)^{2}-M_{+}^{2}}+\left(M_{+}^{2} \rightarrow M_{-}^{2}\right)-2\left(M_{+}^{2} \rightarrow M^{2}\right)\right) \\
= & -4 i g^{2} \int_{0}^{1} d x \int \frac{d^{4} p}{(2 \pi)^{4}} \int \frac{d^{4} k}{(2 \pi)^{4}} \frac{x p^{2}}{p^{2}-m^{2}} \\
= & -4 i g^{2} \int_{0}^{1} d x \int \frac{d^{4} p}{(2 \pi)^{4}} \int \frac{d^{4} k}{(2 \pi)^{4}} \frac{p^{2}}{p^{2}-m^{2}} \frac{(M B)^{2} 6 x^{3}}{\left(k^{2}+x(1-x) p^{2}-M^{2}\right)^{4}} \\
& +O\left(B^{4}\right) .
\end{aligned}
$$

Finally the diagrams (C1), (C2), (C3) with the gauge boson loop in Fig. A.3. The vacuum polarization diagrams of messenger fields is first calculated. Note that the contribution of messenger fermions (C3) is the same as the one in the 
supersymmetric limit, and hence cancels after the subtraction. The scalar loop gives

$$
\begin{aligned}
(\mathrm{C} 1)_{s}= & g^{2} \int \frac{d^{4} k}{(2 \pi)^{4}}(2 k+p)^{\mu}(2 k+p)^{\nu} \\
& {\left[\frac{1}{k^{2}-M_{+}^{2}} \frac{1}{(k+p)^{2}-M_{+}^{2}}+\left(M_{+}^{2} \rightarrow M_{-}^{2}\right)-2\left(M_{+}^{2} \rightarrow M^{2}\right)\right] }
\end{aligned}
$$

and the "seagull" diagram gives

$$
(\mathrm{C} 2)_{s}=-2 g^{2} g^{\mu \nu} \int \frac{d^{4} k}{(2 \pi)^{4}}\left[\frac{1}{k^{2}-M_{+}^{2}}+\left(M_{+}^{2} \rightarrow M_{-}^{2}\right)-2\left(M_{+}^{2} \rightarrow M^{2}\right)\right]
$$

Their sum is

$$
\begin{aligned}
(\mathrm{C} 1)_{s}+(\mathrm{C} 2)_{s}= & g^{2}\left(-g^{\mu \nu} p^{2}+p^{\mu} p^{\nu}\right) \int \frac{d^{4} k}{(2 \pi)^{4}} \int_{0}^{1} d z \frac{6(1-2 z)^{2}(M B)^{2}}{\left(k^{2}+z(1-z) p^{2}-M^{2}\right)^{4}} \\
& +O\left(B^{4}\right)
\end{aligned}
$$

Now including the gauge boson loop, the total contribution of the vacuum polarization diagrams is

$$
(\mathrm{C})_{s}=i 9 g^{2}(M B)^{2} \int \frac{d^{4} k}{(2 \pi)^{4}} \int \frac{d^{4} p}{(2 \pi)^{4}} \int_{0}^{1} d z \frac{p^{2}}{p^{2}-m^{2}} \frac{(1-2 z)^{2}}{\left(k^{2}+z(1-z) p^{2}-M^{2}\right)^{4}} \text {. }
$$

Adding all diagrams, one obtains $-i V_{\text {eff }}\left(m^{2}\right)=(\mathrm{A})_{s}+(\mathrm{B})_{s}+(\mathrm{C})_{s}$ up to $O(M B)^{2}$

$$
V_{e f f}\left(m^{2}\right)=-12 g^{2}(M B)^{2} \int_{0}^{1} d x \int \frac{d^{4} p}{(2 \pi)^{4}} \int \frac{d^{4} k}{(2 \pi)^{4}} \frac{p^{2}}{p^{2}-m^{2}}\left[\frac{(1-2 x)^{2}-2 x^{3}}{\left(k^{2}+x(1-x) p^{2}-M^{2}\right)^{4}}\right]
$$

After a Wick rotation in the $k$ and $p$ spaces, the $d^{4} k$ integration can be carried out trivially. Note also that the denominator is symmetric under the interchange 
of $x \leftrightarrow(1-x)$. Therefore, the $x$ integration is symmetrized by substituting the polynomial in the numerator $P(x)=(1-2 x)^{2}-2 x^{3}$ by $\frac{1}{2}(P(x)+P(1-x))=$ $-x(1-x)$, yielding

$$
V_{e f f}\left(m^{2}\right)=\frac{-g^{2}(M B)^{2}}{128 \pi^{4}} \int_{0}^{1} d x \int_{0}^{\infty} p^{2} d\left(p^{2}\right)\left[\frac{x(1-x) p^{2}}{\left(p^{2}+m^{2}\right)\left(x(1-x) p^{2}+M^{2}\right)^{2}}\right] .
$$

The $p^{2}$ integral is logarithmically divergent. Fortunately, the divergent piece is $m^{2}$ independent, and hence is the renormalization of the cosmological constant. $V_{\text {eff }}(0)$ is subtracted from the above expression and redefined as $V_{\text {eff }}$. The final integral is convergent for any $m^{2} \in[0, \infty)$ :

$$
V_{e f f}\left(m^{2}\right)=\frac{g^{2}(M B)^{2} m^{2}}{128 \pi^{4}} \int_{0}^{1} d x \int_{0}^{\infty} p^{2} d\left(p^{2}\right)\left[\frac{x(1-x)}{\left(p^{2}+m^{2}\right)\left(x(1-x) p^{2}+M^{2}\right)^{2}}\right] .
$$

The $p$ integration can be computed using the following tricks. First, change the integration variable to $q^{2}=x(1-x) p^{2}$. Then the $q^{2}$ integration can be done in the standard way, and one obtains

$$
V_{\text {eff }}\left(z^{2}\right)=\frac{g^{2}(M B)^{2}}{128 \pi^{4}} \int_{0}^{1} d x\left[\frac{1 / z^{2}-x(1-x)+x(1-x) \ln \left[x(1-x) z^{2}\right]}{\left(1 / z^{2}-x(1-x)\right)^{2}}\right] .
$$

Here and below, $z^{2} \equiv m^{2} / M^{2}$ is used.

The $x$ integration can be further performed using dilogarithms. Using the roots of the denominator $a \equiv\left(1-\sqrt{1-4 / z^{2}}\right) / 2$ and $1-a$,

$$
V_{e f f}(a)=\frac{g^{2}(M B)^{2}}{128 \pi^{4}} \int_{0}^{1} d x\left[\frac{(x-a)(x-1+a)+x(1-x) \ln \left(\frac{x(1-x)}{a(1-a)}\right)}{(x-a)^{2}(x-1+a)^{2}}\right] .
$$

After the final integral is carried out one is left with an expression for the effective potential as a function of $a$ :

$$
V_{e f f}(a)=\frac{g^{2}(M B)^{2}}{64 \pi^{4}}\left\{\frac{\ln (a(1-a))}{(1-2 a)^{2}}+\frac{1-2 a(1-a)}{(1-2 a)^{3}} \times\right.
$$




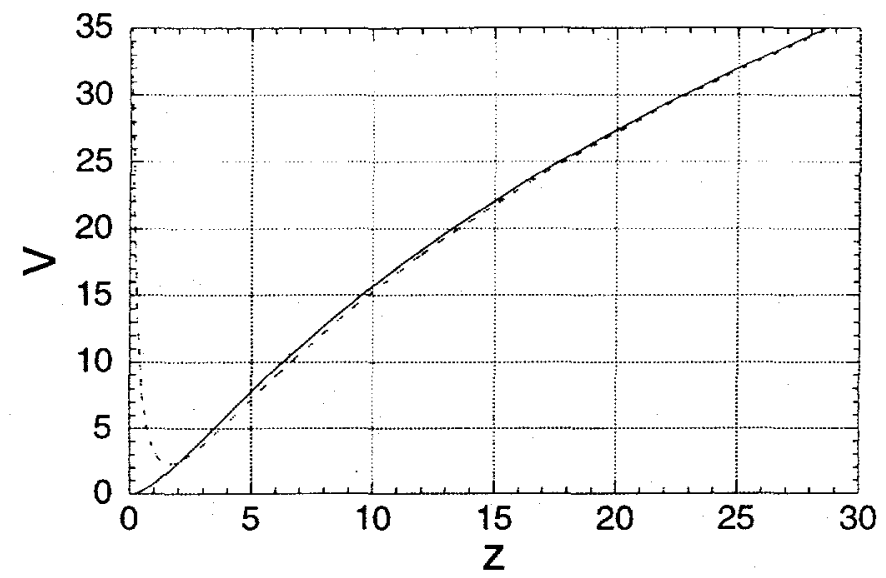

Figure A.4: A plot of the effective potential Eq. (A.15) for $z \equiv 2 g|\phi| / M<30$, in the unit of $g^{2}(M B)^{2} /\left(128 \pi^{4}\right)$. The solid line is the exact result, and the dotted line shows the asymptotic form Eq. (A.16) valid for large $z$.

$$
\left.\left[\frac{1}{2} \ln ^{2}(a)-\frac{1}{2} \ln ^{2}(1-a)-\mathrm{Li}_{2}(a)+\operatorname{Li}_{2}(1-a)\right]\right\} .
$$

The form of the effective potential is shown in Fig. A.4 as a function of $z$ as a solid line.

The expression is manifestly real for $z^{2} \geq 4$. In the limit $z \rightarrow \infty, a \approx 1 / z^{2}$ and the potential behaves as

$$
\begin{aligned}
V_{\text {eff }} & =\frac{g^{2}(M B)^{2}}{64 \pi^{4}}\left[\frac{1}{2} \ln ^{2}\left(z^{2}\right)-\ln \left(z^{2}\right)+\frac{\pi^{2}}{6}+O\left(\frac{1}{z^{2}} \ln ^{2} z^{2}\right)\right] . \\
& \sim \frac{g^{2}(M B)^{2}}{128 \pi^{4}}\left(\ln \frac{4 g^{2}|\phi|^{2}}{e M^{2}}\right)^{2} .
\end{aligned}
$$

This asymptotic form of the effective potential is also shown in Fig. A.4 as a dotted line.

In the case $z^{2}<4, a$ is complex, $a=1 / 2+i \sqrt{4 / z^{2}-1}$. The effective potential 
can be made manifestly real, using the following dilog relations:

$$
\begin{aligned}
\mathrm{Li}_{2}(a)+\frac{1}{2} \ln ^{2}(a) & =-\mathrm{Li}_{2}\left(\frac{1}{a}\right)-i \pi \ln (a)+\frac{\pi^{2}}{2}-\zeta(2), \\
\mathrm{Li}_{2}(1-a)+\frac{1}{2} \ln ^{2}(1-a) & =-\mathrm{Li}_{2}\left(\frac{1}{1-a}\right)+i \pi \ln (1-a)+\frac{\pi^{2}}{2}-\zeta(2)(\mathrm{A} .18)
\end{aligned}
$$

One finds

$$
\begin{aligned}
& V_{e f f}\left(z^{2}<4\right)=\frac{g^{2}(M B)^{2}}{64 \pi^{4}}\left\{-\frac{\ln \left(\frac{1}{z^{2}}\right)}{\frac{4}{z^{2}}-1}+\frac{\left(1-\frac{2}{z^{2}}\right)}{\left(\frac{4}{z^{2}}-1\right)^{\frac{3}{2}}} \times\right. \\
& \left.\times\left[-\ln \left(\frac{1}{z^{2}}\right)\left(\pi+\arctan \left(-\sqrt{\frac{4}{z^{2}}-1}\right)\right)+i\left(\operatorname{Li}_{2}\left(\frac{1}{a}\right)-\operatorname{Li}_{2}\left(\frac{1}{1-a}\right)\right)\right]\right\} .
\end{aligned}
$$

Note that $\mathrm{Li}_{2}(z)-\mathrm{Li}_{2}\left(z^{*}\right)$ is pure imaginary. In the above form, it is simple to take the limit $z^{2} \rightarrow 0$, and one obtains

$$
\begin{aligned}
V_{e f f} & =\frac{g^{2}(M B)^{2}}{64 \pi^{4}}\left[\frac{z^{2}}{4} \ln \left(z^{2}\right)+\left(\frac{z^{3}}{8}-\frac{z}{4}\right)\left(z \ln \left(z^{2}\right)+\frac{i z^{2}}{2}-z-\frac{i z^{2}}{2}-z\right)+O\left(z^{3} \ln \left(z^{2}\right)\right)\right] \\
& =\frac{g^{2}(M B)^{2}}{64 \pi^{4}}\left(\frac{z^{2}}{2}+O\left(z^{3} \ln \left(z^{2}\right)\right)\right) \\
& =\frac{g^{2} m^{2}}{128 \pi^{4}}\left(\frac{(M B)^{2}}{M^{2}}\right)+O\left(m^{4}\right) \\
& =4\left(\frac{\alpha}{4 \pi}\right)^{2}\left(\frac{M B}{M}\right)^{2}\left(|\phi|^{2}+|\bar{\phi}|^{2}\right)+O\left(\phi^{4}\right) .
\end{aligned}
$$

The approximate form Eq. (A.20) truncated at $O\left(z^{2}\right)$ is shown in Fig. A.5 as a dotted line together with the exact form Eq. (A.15) (or equivalently, Eq. (A.19)) as a solid line. From the last expression (A.21) with $\alpha \equiv g^{2} / 4 \pi$, one can read off the mass of the flat direction. For messengers in $5+5^{*}$ representation, the final result is multiplied by a group theory factor $T^{a} T^{b} \operatorname{tr}\left(T^{a} T^{b}\right)=\frac{1}{2} C_{f}$ where the trace is taken over the messenger fields and $C_{f}$ is the second order Casimir for the flat direction. One obtains $m_{\phi}^{2}=2 C_{f}\left(\frac{\alpha}{4 \pi}\right)^{2}\left(\frac{M B}{M}\right)^{2}$, which agrees with that in Ref. [74] 


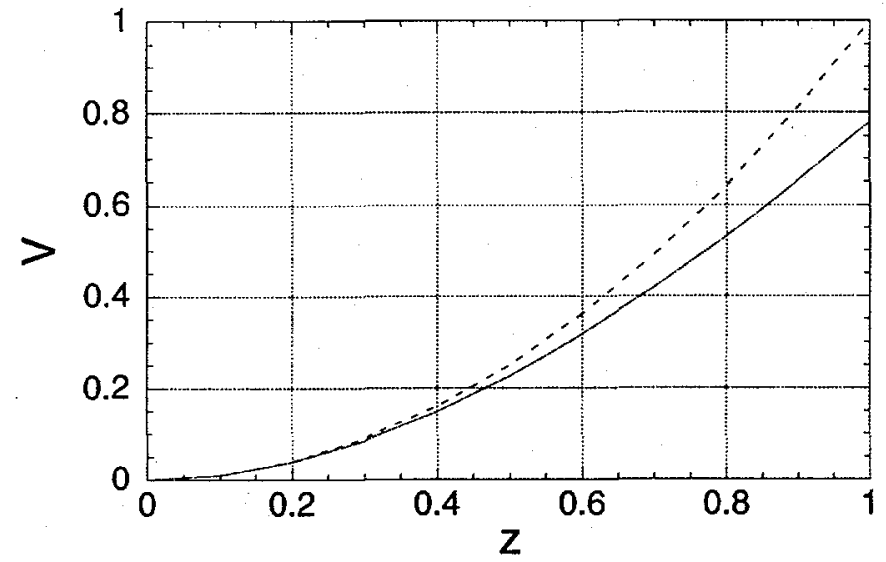

Figure A.5: A plot of the effective potential Eq. (A.15) (or equivalently, Eq. (A.19)) for the small field amplitude, $z \equiv 2 g|\phi| / M<1$, in the unit of $g^{2}(M B)^{2} /\left(123 \pi^{4}\right)$. The solid line is the exact result, and the dotted line shows the approximate form Eq. (A.20) valid for small $z$. 


\section{Appendix B}

\section{Effective Potential and Wave-function}

\section{Renormalization}

When one computes an effective potential, one can determine the location of the minimum. It is well-known that one also needs to evaluate the wave-function renormalization $Z(\phi)(\partial \phi)^{2}$ in order to discuss the time evolution of the scalar field in general. Fortunately, such a calculation is not necessary in the case of interest.

First, recall the simple fact that the effective potential in the case at hand comes at 2-loop order: $V \sim(\alpha / 4 \pi)^{2}$. Since it is a flat direction in the supersymmetric limit, this is the only term in the potential. The equation of motion in the flat space is

$$
\ddot{\phi}+\frac{Z^{\prime}(\phi)}{Z(\phi)}(\dot{\phi})^{2}+\frac{1}{Z(\phi)} V^{\prime}(\phi)=0
$$

Here the friction term $3 H \dot{\phi}$ is dropped, but the essence of the following discussions does not depend on such simplifying assumptions.

Because $V^{\prime}$ is of order $(\alpha / 4 \pi)^{2}$, the motion is suppressed by a power in the coupling constant. Note that $Z^{\prime}(\phi)$ is at most order $(\alpha / 4 \pi)$. By factoring out the coupling constant factors,

$$
V=\left(\frac{\alpha}{4 \pi}\right)^{2} v(\phi)
$$




$$
Z=1+\left(\frac{\alpha}{4 \pi}\right) \zeta(\phi)
$$

one finds

$$
\ddot{\phi}+\left(\frac{\alpha}{4 \pi}\right) \frac{\zeta^{\prime}(\phi)}{Z(\phi)}(\dot{\phi})^{2}+\frac{1}{Z(\phi)}\left(\frac{\alpha}{4 \pi}\right)^{2} v^{\prime}(\phi)=0
$$

It is convenient to rescale the time variable $t$ by

$$
\tau \equiv \frac{\alpha}{4 \pi} t
$$

and one finds

$$
\frac{\partial^{2} \phi}{\partial \tau^{2}}+\left(\frac{\alpha}{4 \pi}\right) \frac{\zeta^{\prime}(\phi)}{Z(\phi)}\left(\frac{\partial \phi}{\partial \tau}\right)^{2}+\frac{1}{Z(\phi)} v^{\prime}(\phi)=0
$$

It is clear that the leading terms in the equation of motion are given by $\partial^{2} \phi / \partial \tau^{2}+$ $v^{\prime}(\phi)=0$, and all dependences on the wave function renormalization occur only at higher orders in perturbation theory. Therefore, the calculation of the effective potential is enough for the case considered here, and $Z(\phi)$ is not needed. 


\section{Appendix $\mathrm{C}$}

\section{Time Evolution of the Flat Direction}

The evolution of the flat direction $\phi$ is interesting in the LEGM models. Once the amplitude is dominated by the gauge-mediated piece, the potential is approximately proportional to $\left(\ln |\phi|^{2}\right)^{2}$, and the dilution of the coherent oscillation occurs much slower than in the parabolic potential case. In this Appendix, the evolution of the flat direction is investigated by using the virial theorem.

The virial theorem states that,

$$
2\langle K\rangle=\left\langle\frac{\partial V}{\partial \phi} \phi+\frac{\partial V}{\partial \phi^{*}} \phi^{*}\right\rangle
$$

where $K=\dot{\phi}^{*} \dot{\phi}$ is the kinetic energy. In the case of interest, $V \sim V_{0}\left(\ln |\phi|^{2} /\langle S\rangle^{2}\right)^{2}$ with $\langle S\rangle \sim 3 \times 10^{4} \mathrm{GeV}$, and

$$
\langle K\rangle=\left\langle\frac{2}{\ln |\phi|^{2} /\langle S\rangle^{2}} V\right\rangle
$$

For $\ln |\phi|^{2} /\langle S\rangle^{2} \gg 1$, the energy density of the field is potential dominated.

The field equation is

$$
\ddot{\phi}+3 H \dot{\phi}+\frac{\partial V}{\partial \phi^{*}}=0
$$

Multiplying it by $\dot{\phi}^{*}$ and using the energy density $\mathcal{E}=K+V$,

$$
0=\dot{\mathcal{E}}+6 H K \sim \dot{\mathcal{E}}+6 H \frac{2}{\ln |\phi|^{2} /\langle S\rangle^{2}} \mathcal{E}
$$


Since the energy density is dominated by the potential term, one can write down the approximate evolution equation of the amplitude $|\phi|$,

$$
\frac{d|\phi|}{d t} \sim-3 H|\phi|,
$$

and hence

$$
|\phi(t)| R(t)^{3} \sim \text { constant }
$$

This formula is valid when $\ln |\phi|^{2} /\langle S\rangle^{2} \gg 1$. 


\section{Appendix D}

\section{Estimation of the Primordial Baryon Asymmetry}

In this Appendix, Linde's formula, Eq. (4.5.6) is justified in the case of interest. In fact, the validity of the formula depends on the nature of the operator $\mathcal{O}$ and the time evolution of the flat direction $\phi$. The reason why the formula is valid is clarified in the cases of interest. Start from the equation of motion for the baryon number density, Eq. (4.5.5),

$$
\dot{n}_{B}+3 H n_{B}=i\left(\frac{\partial \mathcal{O}}{\partial \phi} \phi-\frac{\partial \mathcal{O}}{\partial \phi^{*}} \phi^{*}\right)
$$

It is useful to rewrite the equation in terms of baryon-to-entropy ratio $Y_{B} \equiv n_{B} / s$, to find

$$
\dot{Y}_{B}=\frac{1}{s} i\left(\frac{\partial \mathcal{O}}{\partial \phi} \phi-\frac{\partial \mathcal{O}}{\partial \phi^{*}} \phi^{*}\right)
$$

where the relation $s R^{3}=$ constant is assumed. Assuming a vanishing initial value $Y_{B}\left(t_{0}\right)=0$, one obtains

$$
Y_{B}(\infty)=\int_{t_{0}}^{\infty} d t \frac{1}{s} i\left(\frac{\partial \mathcal{O}}{\partial \phi} \phi-\frac{\partial \mathcal{O}}{\partial \phi^{*}} \phi^{*}\right)
$$

A crucial question is whether the $t$ integral is dominated by $t \sim t_{0}$ or $t \sim \infty$.

In the following analysis, the Universe is assumed to be radiation dominated when the field begins to roll down the potential, $R \propto t^{1 / 2}$. Another assumption is that the baryon-number violating operator $\mathcal{O}$ can be treated as a small 
perturbation to the evolution of the field $\phi$.

In the case $\phi_{0} \gtrsim \phi_{\text {eq }}$, or in the general hidden sector case, $\mathcal{O} \propto \phi^{4}$. The evolution of $\phi$ is essentially determined by $m_{3 / 2}^{2} \phi^{2}$ by assumption and hence $\phi \propto$ $R^{-3 / 2} \propto t^{-3 / 4}$. On the other hand, $s \propto R^{-3} \propto t^{-3 / 2}$. The integrand in Eq. (D.3) therefore behaves as $t^{-3 / 2}$ and hence it is dominated by $t \sim t_{0}$. By putting them together,

$$
\begin{aligned}
Y_{B}(\infty) & =\int_{t_{0}}^{\infty} d t \frac{1}{s_{0}} i\left(\frac{\partial \mathcal{O}}{\partial \phi} \phi-\frac{\partial \mathcal{O}}{\partial \phi^{*}} \phi^{*}\right) \frac{t^{-3 / 2}}{t_{0}^{-3 / 2}} \\
& =\frac{1}{2} t_{0} \times \frac{8 m_{3 / 2}^{2} \operatorname{Im}\left(\phi_{0}^{4}\right)}{s_{0} M_{*}^{2}}=\frac{2 m_{3 / 2}^{2} \operatorname{Im}\left(\phi_{0}^{4}\right)}{s_{0} M_{*}^{2} H_{0}},
\end{aligned}
$$

which essentially justifies Eq. (4.5.6).

In the other case of interest, $\phi_{0} \lesssim \phi_{\text {eq }}$, both the behavior of the operator and time-evolution are completely different as discussed in detail in Sec. 4.5. One has $\mathcal{O} \propto \phi^{2}$, while $\phi \propto R^{-3} \propto t^{-3 / 2}$. Then the integrand behaves as $t^{-3 / 2}$, which is unexpectedly the same as in the previous case. By putting them together, one obtains

$$
\begin{aligned}
Y_{B}(\infty) & =\int_{t_{0}}^{\infty} d t \frac{1}{s_{0}} i\left(\frac{\partial \mathcal{O}}{\partial \phi} \phi-\frac{\partial \mathcal{O}}{\partial \phi^{*}} \phi^{*}\right) \frac{t^{-3 / 2}}{t_{0}^{-3 / 2}} \\
& =\frac{1}{2} t_{0} \times \frac{8 V_{0} \operatorname{Im}\left(\phi_{0}^{4}\right)}{s_{0}\left|\phi_{0}\right|^{2} M_{*}^{2}}=\frac{2 V_{0} \operatorname{Im}\left(\phi_{0}^{4}\right)}{s_{0}\left|\phi_{0}\right|^{2} M_{*}^{2} H_{0}},
\end{aligned}
$$

which again essentially justifies Eq. (4.5.6).

As it is clear from above the derivations, Eq. (4.5.6) is not necessarily valid if the integral is dominated at $t \sim \infty$ rather than $t \sim t_{0}$. We have not seen an explicit discussion on this point in the literature. 


\section{Appendix E}

\section{Affleck-Dine Baryogenesis in Hidden Sector}

\section{Scenarios}

In this appendix, the Affleck-Dine baryogenesis based on the scenario with SUSY breaking in the hidden sector is discussed. In this case, the gravitino mass is much larger than in the LEGM case, and all the scalar fields also have SUSY breaking masses of the order of the gravitino mass. ${ }^{*}$ In particular, the potential for the flat direction is always given by the supergravity contribution, which is essentially parabolic with a curvature of the order of the gravitino mass,

$$
V(\phi) \sim m_{3 / 2}^{2}|\phi|^{2}
$$

with $m_{3 / 2} \sim 1 \mathrm{TeV}$. Due to this fact, the evolution of the flat direction is much simpler than in the LEGM case.

Even if the gravitino mass is about $1 \mathrm{TeV}$, Eq.(4.5.6) is still valid since the baryon number is generated when $\phi$ starts to oscillate. With the baryon number violating operator (4.5.4), one obtains

$$
\left.n_{B}\right|_{H \sim m_{3 / 2}} \sim \frac{m_{3 / 2}\left|\phi_{0}\right|^{4}}{M_{*}^{2}} \sin 4 \theta_{0} .
$$

${ }^{*}$ In this Appendix, all the soft SUSY breaking masses for the scalar fields are denoted by $m_{3 / 2}$ for simplicity. 
and hence

$$
\left.\frac{n_{B}}{s}\right|_{H \sim m_{3 / 2}} \sim g_{*}^{-1 / 4} \frac{\left|\phi_{0}\right|^{4}}{m_{3 / 2}^{1 / 2} M_{*}^{7 / 2}} \sin 4 \theta_{0}
$$

If there is no entropy production after this stage, the above formula yields the resulting baryon-to-entropy ratio.

If there is entropy production, the primordial baryon number is diluted. The primary source of the entropy is the decay of the flat direction. Here, the potential for the flat direction is always parabolic, and $\phi$ starts to oscillate when $T=T_{0} \sim$ $g_{*}^{-1 / 4} \sqrt{m_{3 / 2} M_{*}}$, as discussed in Sec. 4.5. Then, by using the relation $|\phi|^{2} T^{-3}=$ constant, the background temperature at the $\phi$ decay is given by

$$
T_{\mathrm{dec}} \sim g_{*}^{-1 / 4} \sqrt{m_{3 / 2} M_{*}}\left(\frac{\phi_{\mathrm{dec}}}{\left|\phi_{0}\right|}\right)^{2 / 3}
$$

where $\phi_{\mathrm{dec}}$ is the amplitude of the flat direction when it decays. Furthermore, the reheating temperature due to the decay of $\phi, T_{R}$, is given by

$$
T_{\mathrm{R}} \sim g_{*}^{-1 / 4} \sqrt{m_{3 / 2} \phi_{\mathrm{dec}}}
$$

Then, the dilution factor is given by

$$
D \sim \frac{T_{\mathrm{R}}^{3}}{T_{\mathrm{dec}}^{3}} \sim \frac{\left|\phi_{0}\right|^{2}}{\phi_{\mathrm{dec}}^{1 / 2} M_{*}^{3 / 2}}
$$

Usually, $\phi$ decays when the expansion rate of the Universe, $H$, becomes comparable to the decay rate of $\phi, \Gamma_{\phi}$. In Ref.[80], $\Gamma_{\phi}$ is estimated as

$$
\Gamma_{\phi} \sim\left(\frac{\alpha_{s}}{\pi}\right)^{2} \frac{m_{3 / 2}^{3}}{|\phi|^{2}}
$$


and hence $H \sim \Gamma_{\phi}$ results in

$$
\phi_{\mathrm{dec}} \sim\left(\frac{\alpha_{s}}{\pi}\right)^{2 / 3} m_{3 / 2}^{2 / 3} M_{*}^{1 / 3} .
$$

Combining the above results, one obtains

$$
\begin{aligned}
\frac{n_{B}}{s} \sim & \left(\frac{\alpha_{s}}{\pi}\right)^{1 / 3}\left(\frac{M_{*}}{m_{3 / 2}}\right)^{1 / 6}\left(\frac{\left|\phi_{0}\right|}{M_{*}}\right)^{2} \sin 4 \theta_{0} \\
& =120 \times\left(\frac{\left|\phi_{0}\right|}{M_{*}}\right)^{2}\left(\frac{\alpha_{s}}{0.1}\right)^{1 / 3}\left(\frac{m_{3 / 2}}{1 \mathrm{TeV}}\right)^{-1 / 6} \sin 4 \theta_{0} .
\end{aligned}
$$

Another potential source of entropy is the Polonyi field related to SUSY breaking, or the moduli fields in the string theory, which also have masses of order $m_{3 / 2}$. The critical difference between the flat direction and the Polonyi field $z$ is the formula of their decay width; since the Polonyi field couples to particles in the observable sector only through interactions suppressed by the gravitational scale, its decay width $\Gamma_{z}$ is much smaller than the width of $\phi$. As discussed in Sec. 4.6, $\Gamma_{z}$ is estimated as

$$
\Gamma_{z} \sim \frac{N_{\mathrm{ch}}}{4 \pi} \frac{m_{3 / 2}^{3}}{M_{*}^{2}}
$$

Even with this decay rate, one can apply an argument similar to the case of the entropy production due to $\phi$ decay; Eqs.(E.2) and (E.3) are still valid, and one also obtains equations similar to Eqs.(E.4) - (E.6) where $\phi$ 's are replace by $z$ 's. The remainder is to evaluate the amplitude of $z$ at its decay time, $z_{\text {dec }}$, by using the relevant formula for $\Gamma_{z}$. By solving the equation $H \sim \Gamma_{z}$ with $H \sim m_{3 / 2} z / M_{*}$, one obtains

$$
z_{\mathrm{dec}} \sim \frac{m_{3 / 2}^{2}}{M_{*}}
$$


Then, assuming the initial amplitude of $z$ to be $z_{0} \sim M_{*}$, the dilution factor is given by $D \sim M_{*} / m_{3 / 2}$, and hence

$$
\frac{n_{B}}{s} \sim D^{-1} \frac{m_{3 / 2} \operatorname{Im}\left(\phi_{0}^{4}\right)}{M_{*}^{2}} \sim\left(\frac{m_{3 / 2}}{M_{*}}\right)^{1 / 2}\left(\frac{\left|\phi_{0}\right|^{4}}{M_{*}^{4}}\right) \sin 4 \theta_{0}
$$

Thus, the baryon-to-entropy ratio may be larger than $\sim 10^{-10}$ even after the decay of the Polonyi field.

Howeyer the reheating temperature after the decay of $z$ is likely to be too low. By using Eq. (E.10), the reheating temperature is estimated as

$$
T_{\mathrm{R}} \sim g_{*}^{-1 / 4} \sqrt{\Gamma_{z} M_{*}} \sim 1 \mathrm{MeV} \times\left(\frac{m_{3 / 2}}{10 \mathrm{TeV}}\right)^{3 / 2}
$$

Thus, if the gravitino mass is heavier than about $10 \mathrm{TeV}$, the Polonyi field may decay before the big-bang nucleosynthesis (BBN), and the scenario which gives Eq.(E.12) may be viable. ${ }^{\dagger}$ However, for a favorable range of the gravitino mass $\left(m_{3 / 2} \lesssim 1 \mathrm{TeV}\right)$, the reheating temperature is less than $100 \mathrm{keV}$ which is lower than the temperature where the big-bang nucleosynthesis (BBN) starts. This means that the decay of $z$ significantly affects the results of the standard BBN scenario. In this case, some mechanism to reduce the energy density of the Polonyi field is needed. A thermal inflation [81] is an interesting candidate for it. The baryon-toentropy ratio in this case is discussed in Sec. 4.6. By using the fact that the ratio

\footnotetext{
${ }^{\dagger}$ In fact, even if $m_{3 / 2} \gtrsim 10 \mathrm{TeV}$, there may still be a problem since the lightest superparticle produced by the decay of the Polonyi field may overclose the Universe $[100,104]$. To solve this difficulty, one may have to accept a much larger gravitino mass, or a scenario in which the lightest superparticle in the MSSM sector is unstable.
} 
of $n_{B}$ to $\rho_{z}$ is constant in time, one obtains

$$
\frac{n_{B}}{s} \sim \frac{\rho_{z}}{s} \times m_{3 / 2}^{-1}\left(\frac{\left|\phi_{0}\right|}{M_{*}}\right)^{4} \sin 4 \theta_{0} .
$$

Thus, once the ratio $\rho_{z} / s$ after the late inflation is fixed, the baryon-to-entropy ratio can be estimated. 


\section{Appendix $\mathbf{F}$}

\section{The Renormalization Group Equations of the}

\section{(N)MSSM .}

In this appendix, all of the RG equations for the NMSSM (and the MSSM) are presented, at 1-loop [146]. These are the equations used, in Sec. 5.4, to determine the coupling constants and SUSY-breaking parameters of the NMSSM at the weak scale, given their values at the messenger scale.

$$
\begin{aligned}
16 \pi^{2} \frac{d}{d t} g^{\prime} & =11 g^{\prime 3}, \\
16 \pi^{2} \frac{d}{d t} g_{2} & =g_{2}^{3} \\
16 \pi^{2} \frac{d}{d t} g_{3} & =(-3) g_{3}^{3} \\
16 \pi^{2} \frac{d}{d t} h_{t} & =\left(6 h_{t}^{2}+h_{b}^{2}+\lambda^{2}-\frac{13}{9} g^{2}-3 g_{2}^{2}-\frac{16}{3} g_{3}^{2}\right) h_{t}, \\
16 \pi^{2} \frac{d}{d t} h_{b} & =\left(6 h_{b}^{2}+h_{t}^{2}+h_{\tau}^{2}+\lambda^{2}-\frac{7}{9} g^{2}-3 g_{2}^{2}-\frac{16}{3} g_{3}^{2}\right) h_{b}, \\
16 \pi^{2} \frac{d}{d t} h_{\tau} & =\left(4 h_{\tau}^{2}+3 h_{b}^{2}+\lambda^{2}-3 g^{2}-3 g_{2}^{2}\right) h_{\tau}, \\
16 \pi^{2} \frac{d}{d t} \lambda & =\left(4 \lambda^{2}+2 k^{2}+3 h_{t}^{2}+3 h_{b}^{2}+h_{\tau}^{2}-g^{2}-3 g_{2}^{2}\right) \lambda, \\
16 \pi^{2} \frac{d}{d t} k & =6\left(\lambda^{2}+k^{2}\right) k .
\end{aligned}
$$

In the above equations $g^{\prime}$ is the $U(1)_{Y}$ gauge coupling; explicitly $g^{\prime}=e / \cos \theta_{W} \cdot g_{2}$ and $g_{3}$ are, respectively, the weak and strong coupling constants. One defines $g_{1}$ to be the hypercharge coupling constant in the GUT normalization, i.e. $g_{1} \equiv \sqrt{\frac{5}{3}} g^{\prime}$ 
and $\alpha_{1} \equiv \frac{5}{3} \alpha^{\prime}$. Gauge couplings at the messenger scale are defined in such a way that they match their experimental values at the $Z$-mass. Only the effect of third generation Yukawa couplings, namely, $h_{t}, h_{b}$ and $h_{\tau}$, were considered. In the case of the MSSM, $k=\lambda \equiv 0$.

$$
\begin{aligned}
16 \pi^{2} \frac{d}{d t} A_{u_{a}} & =6 h_{t}^{2}\left(1+\delta_{a 3}\right) A_{t}+2 h_{b}^{2} \delta_{a 3} A_{b}+2 \lambda^{2} A_{\lambda} \\
& -4\left(\frac{13}{18} g^{2} M_{1}+\frac{3}{2} g_{2}^{2} M_{2}+\frac{8}{3} g_{3}^{2} M_{3}\right) \\
16 \pi^{2} \frac{d}{d t} A_{d_{a}} & =6 h_{b}^{2}\left(1+\delta_{a 3}\right) A_{b}+2 h_{t}^{2} \delta_{a 3} A_{t}+2 h_{\tau}^{2} \delta_{a 3} A_{\tau}+2 \lambda^{2} A_{\lambda} \\
& -4\left(\frac{7}{18} g^{\prime 2} M_{1}+\frac{3}{2} g_{2}^{2} M_{2}+\frac{8}{3} g_{3}^{2} M_{3}\right) \\
16 \pi^{2} \frac{d}{d t} A_{e_{a}} & =2 h_{\tau}^{2}\left(1+3 \delta_{a 3}\right) A_{\tau}+6 h_{b}^{2} A_{b}+2 \lambda^{2} A_{\lambda} \\
& -6\left(g^{2} M_{1}+g_{2}^{2} M_{2}\right), \\
16 \pi^{2} \frac{d}{d t} A_{\lambda} & =8 \lambda^{2} A_{\lambda}-4 k^{2} A_{k}+6 h_{t}^{2} A_{t}+6 h_{b}^{2} A_{b}+2 h_{\tau}^{2} A_{\tau} \\
& -2\left(g^{2} M_{1}+3 g_{2}^{2} M_{2}\right), \\
16 \pi^{2} \frac{d}{d t} A_{k} & =12\left(k^{2} A_{k}-\lambda^{2} A_{\lambda}\right) .
\end{aligned}
$$

$A_{i}$ are the soft SUSY-breaking trilinear couplings, given in Secs. 5.2 and 5.4. Note that only third generation trilinear couplings, namely $A_{t} h_{t}=\mathcal{A}_{u}^{33}, A_{b} h_{b}=\mathcal{A}_{d}^{33}$, $A_{\tau} h_{\tau}=\mathcal{A}_{l}^{33}$ are considered. $M_{i}(i=1,2,3)$ are the soft SUSY-breaking gaugino masses and they evolve, at one loop, identically to $\alpha_{i}$. Explicitly

$$
\frac{M_{i}(Q)}{M_{\frac{1}{2}}}=\frac{g_{i}^{2}(Q)}{g_{X}^{2}}
$$

where $g_{X}$ is the value of all $g_{i}$ at the GUT scale, while $M_{\frac{1}{2}}$ is the common gaugino 
mass at the GUT scale. In the case of the MSSM, $A_{k}=A_{\lambda} \equiv 0$.

$$
\begin{aligned}
& 16 \pi^{2} \frac{d}{d t} m_{\tilde{Q}_{a}}^{2}=2 \delta_{a 3} h_{t}^{2}\left(m_{\tilde{Q}_{3}}^{2}+m_{H_{u}}^{2}+m_{\tilde{t}}^{2}+A_{t}^{2}\right)+2 \delta_{a 3} h_{b}^{2}\left(m_{\tilde{Q}_{3}}^{2}+m_{H_{d}}^{2}+m_{\tilde{b}}^{2}+A_{b}^{2}\right) \\
& \text { - } 8\left(\frac{1}{36} g^{\prime 2} M_{1}^{2}+\frac{3}{4} g_{2}^{2} M_{2}^{2}+\frac{4}{3} g_{3}^{2} M_{3}^{2}\right)+\frac{1}{3} g^{r 2} \xi, \\
& 16 \pi^{2} \frac{d}{d t} m_{\tilde{u}_{a}}^{2}=4 \delta_{a 3} h_{t}^{2}\left(m_{\tilde{Q}_{3}}^{2}+m_{H_{u}}^{2}+m_{\tilde{t}}^{2}+A_{t}^{2}\right) \\
& -8\left(\frac{4}{9} g^{\prime 2} M_{1}^{2}+\frac{4}{3} g_{3}^{2} M_{3}^{2}\right)-\frac{4}{3} g^{2} \xi \\
& 16 \pi^{2} \frac{d}{d t} m_{\tilde{d}_{a}}^{2}=4 \delta_{a 3} h_{b}^{2}\left(m_{\tilde{Q}_{3}}^{2}+m_{H_{d}}^{2}+m_{\tilde{b}}^{2}+A_{b}^{2}\right) \\
& -8\left(\frac{1}{9} g^{2} M_{1}^{2}+\frac{4}{3} g_{3}^{2} M_{3}^{2}\right)+\frac{2}{3} g^{\prime 2} \xi \\
& 16 \pi^{2} \frac{d}{d t} m_{\tilde{L}_{a}}^{2}=2 \delta_{a 3} h_{\tau}^{2}\left(m_{\tilde{L}_{3}}^{2}+m_{H_{d}}^{2}+m_{\tilde{\tau}}^{2}+\dot{A}_{\tau}^{2}\right) \\
& \text { - } 8\left(\frac{1}{4} g^{\prime 2} M_{1}^{2}+\frac{3}{4} g_{2}^{2} M_{2}^{2}\right)-g^{\prime 2} \xi \\
& 16 \pi^{2} \frac{d}{d t} m_{\tilde{e}_{a}}^{2}=4 \delta_{a 3} h_{\tau}^{2}\left(m_{\tilde{L}_{3}}^{2}+m_{H_{d}}^{2}+m_{\tilde{\tau}}^{2}+A_{\tau}^{2}\right) \\
& -8 g^{2} M_{1}^{2}+2 g^{2} \xi, \\
& 16 \pi^{2} \frac{d}{d t} m_{H_{d}}^{2}=6 h_{b}^{2}\left(m_{\tilde{Q}_{3}}^{2}+m_{H_{d}}^{2}+m_{\tilde{b}}^{2}+A_{b}^{2}\right)+2 h_{\tau}^{2}\left(m_{\tilde{L}_{3}}^{2}+m_{H_{d}}^{2}+m_{\tilde{\tau}}^{2}+A_{\tau}^{2}\right) \\
& +2 \lambda^{2}\left(m_{H_{d}}^{2}+m_{H_{u}}^{2}+m_{N}^{2}+A_{\lambda}^{2}\right) \\
& \text { - } 8\left(\frac{1}{4} g^{\prime 2} M_{1}^{2}+\frac{3}{4} g_{2}^{2} M_{2}^{2}\right)-g^{\prime 2} \xi \\
& 16 \pi^{2} \frac{d}{d t} m_{H_{u}}^{2}=6 h_{t}^{2}\left(m_{\tilde{Q}_{3}}^{2}+m_{H_{u}}^{2}+m_{\tilde{t}}^{2}+A_{t}^{2}\right)+2 \lambda^{2}\left(m_{H_{d}}^{2}+m_{H_{u}}^{2}+m_{N}^{2}+A_{\lambda}^{2}\right) \\
& -8\left(\frac{1}{4} g^{\prime 2} M_{1}^{2}+\frac{3}{4} g_{2}^{2} M_{2}^{2}\right)+g^{\prime 2} \xi \\
& 16 \pi^{2} \frac{d}{d t} m_{N}^{2}=4 \lambda^{2}\left(m_{H_{d}}^{2}+m_{H_{u}}^{2}+m_{N}^{2}+A_{\lambda}^{2}\right)+4 k^{2}\left(3 m_{N}^{2}+A_{k}^{2}\right) \text {. }
\end{aligned}
$$

$\xi$ is the hypercharge-weighted sum of all soft SUSY-breaking masses-squared

$$
\xi=\sum_{i} Y_{i} m_{i}^{2}
$$


where $i$ runs over all scalar particles. With the boundary conditions in Eqs. (5.2.5,5.2.6), $\xi=0$ and remains zero throughout the RG evolution. All soft SUSY-breaking mass-squared terms were taken to be diagonal. Again, only the running of third generation soft SUSY-breaking masses-squared were considered. $m_{N}^{2}$ is defined in Sec. 5.4. Again, in the case of the MSSM, $m_{N}^{2} \equiv 0$.

The $\mu$ and $B \mu \equiv m_{3}^{2}$ parameters of the MSSM obbey

$$
\begin{aligned}
16 \pi^{2} \frac{d}{d t} \mu & =\mu\left(3 h_{t}^{2}+3 h_{b}^{2}+h_{\tau}^{2}-g^{2}-3 g_{2}^{2}\right) \\
16 \pi^{2} \frac{d}{d t} B & =-2\left(3 h_{t}^{2} A_{t}+3 h_{b}^{2} A_{b}+h_{\tau}^{2} A_{\tau}-g^{\prime 2} M_{1}-3 g_{2}^{2} M^{2}\right) .
\end{aligned}
$$




\section{Appendix G}

\section{Scalar Higgs Mass-Squared Matrix}

In this appendix, the $3 \times 3$ scalar Higgs mass-squared matrix of the NMSSM is explicitly shown.

$$
\begin{aligned}
& \mathcal{M}_{\text {scalar }}^{2}=\frac{1}{2} \frac{\partial^{2} V_{\text {tree }}}{\partial v_{i} \partial v_{j}}=\frac{1}{2} \times \\
& \left(\begin{array}{ccc}
\bar{g}^{2} v_{1}^{2}+\left(A_{\lambda}+\frac{k \mu}{\lambda}\right) \frac{2 \mu v_{2}}{v_{1}} & \left(4 \lambda^{2}-\bar{g}^{2}\right) v_{1} v_{2}-2 \mu\left(A_{\lambda}+\frac{k \mu}{\lambda}\right) & 4 \lambda \mu v_{1}-2 A_{\lambda} \lambda v_{2}-4 k \mu v_{2} \\
\left(4 \lambda^{2}-\bar{g}^{2}\right) v_{1} v_{2}-2 \mu\left(A_{\lambda}+\frac{k \mu}{\lambda}\right) & \bar{g}^{2} v_{2}^{2}+\left(A_{\lambda}+\frac{k \mu}{\lambda}\right) \frac{2 \mu v_{1}}{v_{2}} & -2 A_{\lambda} \lambda v_{1}-4 k \mu v_{1}+4 \lambda \mu v_{2} \\
4 \lambda \mu v_{1}-2 A_{\lambda} \lambda v_{2}-4 k \mu v_{2} & -2 A_{\lambda} \lambda v_{1}-4 k \mu v_{1}+4 \lambda \mu v_{2} & -2 A_{k} k \mu \\
\lambda & \frac{8 k^{2} \mu^{2}}{\lambda^{2}}+\frac{2 A_{\lambda} \lambda^{2} v_{1} v_{2}}{\mu}
\end{array}\right),
\end{aligned}
$$

where $v_{i}$ for $i=1,2,3$ corresponds, respectively, to $v_{d}, v_{u}$ and $x$. All other parameters were defined in previous sections.

The determinant of the matrix above can be evaluated explicitly, and its full expression is given bellow. Various limits of this determinant are considered in the body of the chapter.

$$
\begin{aligned}
& \operatorname{det} \mathcal{M}_{\text {scalar }}^{2}= \\
& \frac{v^{2}}{32 \lambda^{3} \mu \sin (2 \beta)}\left(-6 A_{\lambda} v^{4} \lambda^{9}-32 A_{\lambda}^{3} \lambda^{5} \mu^{2}-64 A_{\lambda} v^{2} \lambda^{7} \mu^{2}-32 A_{k} A_{\lambda} k \lambda^{4} \mu^{3}-160 A_{\lambda}^{2} k \lambda^{4} \mu^{3}-\right. \\
& 128 k v^{2} \lambda^{6} \mu^{3}-32 A_{k} k^{2} \lambda^{3} \mu^{4}-128 A_{\lambda} k^{2} \lambda^{3} \mu^{4}-256 A_{\lambda} \lambda^{5} \mu^{4}-256 k \lambda^{4} \mu^{5}+8 A_{\lambda} v^{4} \lambda^{9} \cos (4 \beta)+ \\
& 32 A_{\lambda}^{3} \lambda^{5} \mu^{2} \cos (4 \beta)+64 A_{\lambda} v^{2} \lambda^{7} \mu^{2} \cos (4 \beta)+32 A_{k} A_{\lambda} k \lambda^{4} \mu^{3} \cos (4 \beta)+160 A_{\lambda}^{2} k \lambda^{4} \mu^{3} \cos (4 \beta)+ \\
& 128 k v^{2} \lambda^{6} \mu^{3} \cos (4 \beta)+32 A_{k} k^{2} \lambda^{3} \mu^{4} \cos (4 \beta)+128 A_{\lambda} k^{2} \lambda^{3} \mu^{4} \cos (4 \beta)-2 A_{\lambda} v^{4} \lambda^{9} \cos (8 \beta)+ \\
& 3 A_{\lambda} v^{4} \lambda^{7} \bar{g}^{2}+32 A_{\lambda} v^{2} \lambda^{5} \mu^{2} \bar{g}^{2}-16 A_{k} A_{\lambda} k \lambda^{2} \mu^{3} \bar{g}^{2}+64 k v^{2} \lambda^{4} \mu^{3} \bar{g}^{2}-16 A_{k} k^{2} \lambda \mu^{4} \bar{g}^{2}+ \\
& 64 A_{\lambda} k^{2} \lambda \mu^{4} \bar{g}^{2}+64 k^{3} \mu^{5} \bar{g}^{2}-4 A_{\lambda} v^{4} \lambda^{7} \cos (4 \beta) \bar{g}^{2}-32 A_{\lambda} v^{2} \lambda^{5} \mu^{2} \cos (4 \beta) \bar{g}^{2}- \\
& 16 A_{k} A_{\lambda} k \lambda^{2} \mu^{3} \cos (4 \beta) \bar{g}^{2}-64 k v^{2} \lambda^{4} \mu^{3} \cos (4 \beta) \bar{g}^{2}-16 A_{k} k^{2} \lambda \mu^{4} \cos (4 \beta) \bar{g}^{2}+
\end{aligned}
$$




$$
\begin{aligned}
& 64 A_{\lambda} k^{2} \lambda \mu^{4} \cos (4 \beta) \bar{g}^{2}+64 k^{3} \mu^{5} \cos (4 \beta) \bar{g}^{2}+A_{\lambda} v^{4} \lambda^{7} \cos (8 \beta) \bar{g}^{2}+48 A_{\lambda}^{2} v^{2} \lambda^{7} \mu \sin (2 \beta)+ \\
& 24 A_{k} k v^{2} \lambda^{6} \mu^{2} \sin (2 \beta)+120 A_{\lambda} k v^{2} \lambda^{6} \mu^{2} \sin (2 \beta)+256 A_{\lambda}^{2} \lambda^{5} \mu^{3} \sin (2 \beta)+96 v^{2} \lambda^{7} \mu^{3} \sin (2 \beta)+ \\
& 768 A_{\lambda} k \lambda^{4} \mu^{4} \sin (2 \beta)+512 k^{2} \lambda^{3} \mu^{5} \sin (2 \beta)-12 A_{\lambda}^{2} v^{2} \lambda^{5} \mu \bar{g}^{2} \sin (2 \beta)-12 A_{k} k v^{2} \lambda^{4} \mu^{2} \bar{g}^{2} \sin (2 \beta)- \\
& 60 A_{\lambda} k v^{2} \lambda^{4} \mu^{2} \bar{g}^{2} \sin (2 \beta)-16 k^{2} v^{2} \lambda^{3} \mu^{3} \bar{g}^{2} \sin (2 \beta)-48 v^{2} \lambda^{5} \mu^{3} \bar{g}^{2} \sin (2 \beta)-16 A_{\lambda}^{2} v^{2} \lambda^{7} \mu \sin (6 \beta)- \\
& 8 A_{k} k v^{2} \lambda^{6} \mu^{2} \sin (6 \beta)-40 A_{\lambda} k v^{2} \lambda^{6} \mu^{2} \sin (6 \beta)-32 v^{2} \lambda^{7} \mu^{3} \sin (6 \beta)+4 A_{\lambda}^{2} v^{2} \lambda^{5} \mu \bar{g}^{2} \sin (6 \beta)+ \\
& 4 A_{k} k v^{2} \lambda^{4} \mu^{2} \bar{g}^{2} \sin (6 \beta)+4 A_{\lambda} k v^{2} \lambda^{4} \mu^{2} \bar{g}^{2} \sin (6 \beta)-16 k^{2} v^{2} \lambda^{3} \mu^{3} \bar{g}^{2} \sin (6 \beta)+ \\
& \left.16 v^{2} \lambda^{5} \mu^{3} \bar{g}^{2} \sin (6 \beta)\right) .
\end{aligned}
$$

All parameters were defined previously. Recall that $\mu=\lambda x$. 


\section{Appendix $\mathbf{H}$}

\section{Comments on Naturalness}

The NMSSM with extra vector-like quarks was studied in Sec. 5.5.1 and it was argued that the model requires a delicate cancellation among independent parameters. In this appendix, further comments on the naturalness of this model are made.

From Fig. 5.4, one can easily note that not only does the experimentally allowed value of $v$ lie on a steep region of the parameter space, which requires a degree of cancellation of order $1 \%$, but it lies on the steepest region of the parameter space.

One may, therefore, try to address the following question: if all parameters are kept fixed (and this choice of parameters yields an experimentally allowed spectrum) except one (e.g. $\lambda)$, what is the likelihood of obtaining a certain value of $v$ upon a random choice of the free parameter? In other words, what is the probability $P(v) \mathrm{d} v$ of finding the value of $\sqrt{v_{u}^{2}+v_{d}^{2}}$ between $v$ and $v+\mathrm{d} v$ given a random choice of $\lambda$ ? This line of reasoning is related to the definition of fine-tuning introduced by Anderson and Castaño [147]. It is easy to note that

$$
P(v) \propto\left(\frac{\mathrm{d} v}{\mathrm{~d} \lambda}\right)^{-1}
$$

This "probability density" is plotted in Fig. H.1. Note that $\lambda$ is restricted to lie on a range where the same "qualitative" physics is obtained, that is, electroweak 


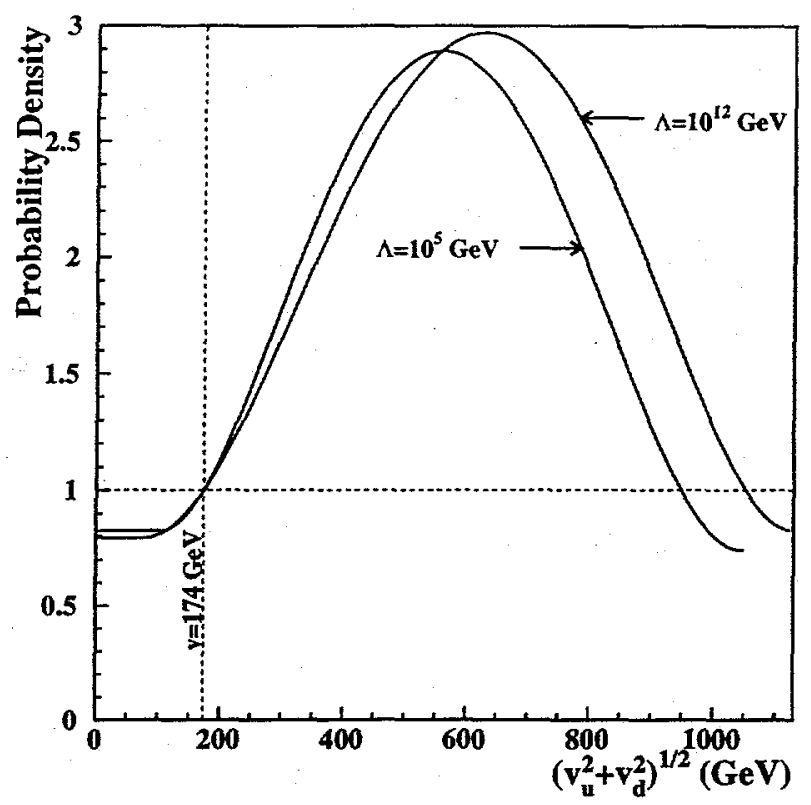

Figure H.1: The probability densities of finding specific values of $v$ in the NMSSM with extra vector-like quarks upon random choices of $\lambda$. All other parameters are the same as in Fig. 5.4. The probability densities are normalized so that $P(v=174 \mathrm{GeV})=1$.

symmetry is broken and $\tan (\beta)>1$. The plot has been normalized in such a way that $P(v=174 \mathrm{GeV})=1$.

It is interesting to note that, in some sense, the probability of living in our universe is smaller, if this model is to be taken seriously, than the probability of living in a universe where $v \simeq 600 \mathrm{GeV}$ by a factor of three. One can turn this picture around and say that the NMSSM, with the above choice of parameters, "prefers" (or predicts) $v \simeq 600 \mathrm{GeV}$.

This does not happen in the MSSM. The analog of Fig. 5.4 would be Eq. (5.3.5), 
which is a straight line $\left(M_{Z}^{2}=M_{Z}^{2}\left(\mu^{2}\right)\right)$ if all parameters except $\mu^{2}$ are kept fixed. In the language introduced above, the MSSM does not "prefer" (or predict) any particular value of $M_{Z}^{2}$, that is, the "probability density" of $M_{Z}^{2}$ upon random choices of $\mu^{2}$ is flat. 


\section{Appendix I}

\section{The Dependence of the Higgs VEVs on the couplings of the modified NMSSM}

In Subsec. 5.5.1 it was shown that the values of the Higgs boson VEVs were extremely sensitive to small variations of the superpotential couplings $\lambda$ and $k$. These variations were evaluated numerically after Eq. (5.5.4) for one particular set of $\lambda$ and $k$. In this appendix, this issue is studied analytically and it is shown that one can estimate the effects of small variations $\Delta \lambda$ and $\Delta k$ on $v, \tan \beta$, and $x$.

The following three equations, derived in Sec. 5.4, are used:

$$
\begin{aligned}
\frac{\bar{g}^{2} v^{2}}{4} & =-\lambda^{2} x^{2}+f \\
2 k^{2} x^{2} & \simeq-m_{N}^{2}+\lambda(k \sin 2 \beta-\lambda) v^{2}, \\
\sin 2 \beta & \simeq 2 \frac{k}{\lambda}\left[\frac{-m_{H_{u}}^{2}}{m_{H_{d}}^{2}-m_{H_{u}}^{2}}\right],
\end{aligned}
$$

where

$$
f \equiv-\frac{1}{2}\left(m_{H_{d}}^{2}+m_{H_{u}}^{2}\right)-\frac{1}{2} \frac{m_{H_{d}}^{2}-m_{H_{u}}^{2}}{\cos 2 \beta}=\frac{m_{H_{d}}^{2}-m_{H_{u}}^{2} \tan ^{2} \beta}{\tan ^{2} \beta-1}
$$

and the $A$-terms in Eq. (I.2) were dropped. Notice that, because the term on the left-hand side of Eq. (I.1) is much smaller then each of the terms on the right-hand side, $f \simeq \lambda^{2} x^{2}$. 
For the purpose of the following estimates only the largest terms in the variations will be kept. According to the numbers presented after Eq. (5.5.4), for a small variation of $\lambda$ the largest variation on the right-hand side of Eq. (I.1) is $2 \lambda x^{2} \Delta \lambda$. One can therefore write

$$
\Delta\left(v^{2}\right) \approx-4 \frac{2 \lambda x^{2} \Delta \lambda}{\bar{g}^{2}}
$$

This approximation will be justified a posteriori. Also, in the following analysis the dependence of the soft-breaking masses-squared on $\lambda$ and $k$ will be completely neglected. This dependence is very weak, as seen in the numbers presented after Eq. (5.5.4).

A small change in $\lambda$ results in a large change in $v$. Hence, to determine the corresponding change in $x$, one can use Eq. (1.2) and only consider the variation of $v^{2}$, which is approximately given by Eq. (I.5). One finds

$$
4 k^{2} x \Delta x \approx \lambda(k \sin 2 \beta-\lambda) \Delta\left(v^{2}\right) \approx \lambda(k \sin 2 \beta-\lambda)\left(-4 \frac{2 \lambda x^{2} \Delta \lambda}{\bar{g}^{2}}\right)
$$

so that

$$
\frac{\Delta x}{x} \approx-\frac{2 \lambda^{3}(k \sin 2 \beta-\lambda)}{k^{2} \bar{g}^{2}} \frac{\Delta \lambda}{\lambda}
$$

For the point considered in the text $(\lambda=0.11, k=-.045, \tan \beta=-2.9)$ one finds $(\Delta x) / x \approx 0.2(\Delta \lambda) / \lambda$.

Under a small change $\Delta k$, again using Eqs. (I.2,I.5),

$$
4\left(k \Delta k x^{2}+k^{2} x \Delta x\right) \approx \lambda(k \sin 2 \beta-\lambda)\left(-4 \frac{2 \lambda^{2} x \Delta x}{\bar{g}^{2}}\right)
$$


Solving for $\Delta x / x$.

$$
\frac{\Delta x}{x} \approx \frac{\Delta k}{k}\left(-1-\frac{2 \lambda^{3}(k \sin 2 \beta-\lambda)}{k^{2} \bar{g}^{2}}\right)^{-1}
$$

Numerically, $(\Delta x) / x \approx-1.2(\Delta k) / k$.

Next, the effect of $\Delta \lambda$ on $f$ is considered. The problem comes down to estimating $\Delta(\cos 2 \beta)^{-1}$, which can be done with the aid of Eq. (I.3):

$$
\Delta(\cos 2 \beta)^{-1}=-\frac{\sin 2 \beta}{\cos ^{3} 2 \beta} \Delta(\sin 2 \beta) \approx-\frac{\sin 2 \beta}{\cos ^{3} 2 \beta}\left(-2 \frac{k}{\lambda^{2}} \frac{-m_{H_{u}}^{2}}{m_{H_{d}}^{2}-m_{H_{u}}^{2}}\right) \Delta \lambda
$$

Thus,

$$
\frac{\Delta f}{f} \approx-\frac{k}{\lambda} \frac{\left(-m_{H_{u}}^{2}\right)}{\lambda^{2} x^{2}} \frac{\sin 2 \beta}{\cos ^{3} 2 \beta} \frac{\Delta \lambda}{\lambda}
$$

Plugging in the numerical values of the parameters, one finds that the right-hand side of Eq. (I.11) equals $-0.5 \Delta \lambda / \lambda$. Thus, a $1 \%$ change in $\lambda$ results in a $0.5 \%$ change in the value of $f$. Since $\lambda^{2} x^{2}$ changes by $2 \%$ in this case, the contribution of $f$ to the variation of $v$ is approximately one fourth of that of $\lambda^{2} x^{2}$, consistent with the numbers given in Subsec. 5.5.1.

The above argument can be repeated to find the effect of $\Delta k$ on $f$. Notice that $\sin 2 \beta$ depends on the ratio $k / \lambda$ (Eq. (I.3)), and hence changing $k$ by $+1 \%$ has the same effect on $f$ as changing $\lambda$ by $-1 \%$.

Finally, it is shown that the condition for $v$ to remain constant is $\Delta k / k=$ $\Delta \lambda / \lambda$. It has already been argued that $\sin 2 \beta$, and therefore $f$, stays unchanged in this case and now it is shown that the same is true for $\lambda^{2} x^{2}$. Under $\lambda \rightarrow \lambda+\Delta \lambda$ the term $\lambda^{2} x^{2}$ changes by $2 \lambda^{2} x^{2}((\Delta \lambda / \lambda)+(\Delta x / x))=2 \lambda^{2} x^{2}(1+0.2)(\Delta \lambda / \lambda)$, while 
under $k \rightarrow k+\Delta k$ it changes by $2 \lambda^{2} x^{2}(\Delta x / x)=2 \lambda^{2} x^{2}(-1.2)(\Delta k / k)$. These variations can be made to cancel by imposing $\Delta k / k=\Delta \lambda / \lambda$. 


\section{Bibliography}

[1] for a pedagogical description see e.g., M.E. Peskin and D.V. Schroeder, "An Introduction to Quantum Field Theory", Addison-Wesley Publishing Co. (1995).

[2] Y. Fukuda et al., Phys. Rev. Lett. 81, 1562 (1998).

[3] C. Caso et al., Eur. Phys. J. C3, 1 (1998).

[4] S. Weinberg, Phys. Rev D19, 1277 (1979); L. Susskind, Phys. Rev D20, 2619 (1979).

[5] N. Arkani-Hamed, S. Dimopoulos and G. Dvali, Phys.Lett. B429,263 (1998).

[6] for a pedagogical introduction see e.g. J. Wess and J. Bagger, "Supersymmetry and Supergravity", Second Edition, Princeton University Press (1992).

[7] For a detailed description of the MSSM, including its particle content and interactions see, e.g. H. Haber and G. Kane, Phys. Rep. 117, 75 (1985); S. Dawson, hep-ph/9712464.

[8] for a recent review on the upper bounds on R-parity violating couplings, see V. Bednyakov, A. Faessler, and S. Kovalenko, hep-ph/9904414.

[9] H. Haber, hep-ph/9901365, to appear in the Proceedings of the Fourth International Symposium on Radiative Corrections (RADCOR 98): Applica- 
tions of Quantum Field Theory to Phenomenology, Universitat Autónoma de Barcelona, Barcelona, Spain, 8-12 September 1998.

[10] M. Chanowitz, Phys. Rev. Lett. 80, 2521 (1998).

[11] J. Ellis and D.V. Nanopoulos, Phys. Lett. B110, 44 (1982); R. Barbieri and R. Gatto, Phys. Lett. B110, 211 (1982); M. Duncan, Nucl. Phys. E221, 285 (1983); J. Donoghue, H.P. Nilles, and D. Wyler, Phys. Lett. B128, 55 (1983); A. Bouquet, J. Kaplan, and C.A. Savoy, Phys. Lett. B148, 69 (1984); L.J. Hall, V. Kostelecky, and S. Raby, Nucl. Phys. B267, 415 (1985).

[12] F. Gabbiani and A. Massiero, Nucl. Phys. B322, 235 (1989).

[13] F. Gabbiani, E. Gabrielli, A. Massiero, and L. Silvestrini, Nucl. Phys. B.477, 321 (1996).

[14] H. Baer et al., LBL-37016, hep-ph/9503479, in "Electroweak Symmetry Breaking and Beyond the Standard Model," eds. T. Barklow, S. Dawson, H. Haber, and J. Siegrist, World Scientific.

[15] S. Lammel, FERMILAB-CONF-98-055-E, hep-ex/9802010.

[16] J.L. Feng, et al., Phys. Rev. D52, 1418 (1995).

[17] D. Descamp, it et al., Phys. Lett. B235, 399 (1990).

[18] D. Dicus, S. Nandi, and X. Tata, Phys. Lett. B129, 451 (1983); A. Chamseddine, P. Nath, and R. Arnowitt, Phys. Lett. B129, 445 (1983). 
[19] I. Hinchliffe, it et al., Phys. Rev. D55, 5520 (1997).

[20] L. Clavelli, Phys. Rev. D46, 2112 (1992).

[21] G. R. Farrar, Phys. Rev. D51, 3904 (1995).

[22] R. M. Barnett, in Proceedings of "Beyond the Standard Model IV," eds. J. F. Gunion, T. Han, and J. Ohnemus, World Scientific, 1995.

[23] H. E. Haber, in Proceedings of the International Workshop on Supersymmetry and Unification of Fundamental Interactions (SUSY 93), ed. Pran Nath, World Scientific, 1993.

[24] B. Adeva et al., Phys. Lett. B 248, 227 (1990).

[25] M. Z. Akrawy et al., Z. Phys. C 49, 49 (1991).

[26] P. Abreu et al., Phys. Lett. B255, 466 (1991).

[27] D. Decamp et al., Phys. Lett. B284, 151 (1992).

[28] P. Abreu et al., Z. Phys C59, 357 (1993).

[29] R. Akers et al., Z. Phys. C65, 367 (1995).

[30] R. M. Barnett et al., to appear in Phys. Rev. D 54, 1 (1996).

[31] G. R. Farrar, Phys. Rev. Lett., 53, 1029 (1984).

[32] S. Dawson et al., Phys. Rev., D31, 1581 (1985). 
[33] F. Bucella, G. R. Farrar, and A. Pugliese, Phys. Lett., B153, 311 (1985).

[34] M. Çakir and G. R. Farrar, Phys. Rev. D 50, 3268 (1994).

[35] C. Albajar et al., Phys. Lett. 198B, 261 (1987).

[36] R. M. Barnett, H. E. Haber, and G.L. Kane, Nucl. Phys. B267, 625 (1986).

[37] L. Clavelli, P.W. Coulter, and K.-j. Yuan, Phys. Rev. D47, 1973 (1993).

[38] L. Clavelli, and P.W. Coulter, Phys. Rev. D51, 1117 (1995).

[39] I. Antoniadis, J. Ellis, and D. V. Nanopoulos, Phys. Lett. B262, 109 (1991).

[40] T. Hebbeker, Z. Phys. C60, 63 (1993).

[41] J. Ellis, D.V. Nanopoulos, and D. A. Ross, Phys. Lett. B305, 375 (1993).

[42] For instance, see I. Hinchliffe in [30].

[43] R.G. Roberts and W.J. Stirling, Phys. Lett. B313, 453 (1993).

[44] J. Blümlein and J. Botts, Phys. Lett. B325, 190 (1994); Erratum, ibid., B331, 450 (1994).

[45] R. Muñoz-Tapia and W.J. Stirling, Phys. Rev. D52, 3984 (1995).

[46] G. R. Farrar, Phys. Lett. B265, 395 (1991).

[47] R. Muñoz-Tapia and W.J. Stirling, Phys. Rev. D49, 3763 (1994).

[48] M. Bengtsson and P.M. Zerwas, Phys. Lett. 208B, 306 (1988). 
[49] O. Nachtmann and A. Reiter, Z. Phys. C16, 45 (1982); M. Bengtsson, Z. Phys. C42, 75 (1989).

[50] A. Ballestrero, E. Maina, and S. Moretti, Phys. Lett. B294, 425 (1992); Nucl. Phys. B415, 265 (1994).

[51] C. D. Carone and H. Murayama, Phys. Rev. Lett. 74, 3122 (1995).

[52] H. Murayama, I. Watanabe, and K. Hagiwara, KEK-91-11, Jan 1992.

[53] A. Djouadi, B. Lampe, and P. M. Zerwas, Z. Phys. C67, 123 (1995).

[54] A. Signer and L. Dixon, Phys. Rev. Lett. 78811 (1997); E.W.N. Glover, and D.J. Miller, Phys. Lett. B396, 257 (1997); Z. Bern, L. Dixon, D. A. Kosower, and S. Weinzierl, Nucl. Phys. B489, 3 (1997).

[55] L. Dixon, private communication.

[56] R. Barate et al., Z. Phys. C76, 1 (1997).

[57] See, e.g., S. Dawson, hep-ph/9712464.

[58] H. Baer et al in "Electroweak Symmetry Breaking and New Physics at the TeV Scale," ed. by T. Barklow et al (World Scientific, Singapore, 1996).

[59] See, e.g., G. Giudice and R. Rattazzi, CERN-TH-97-380, hep-ph/9801271.

[60] D. Lyth and E. Stewart, Phys. Rev. D53, 1784 (1996).

[61] C. Carone, L. Hall and H. Murayama, Phys. Rev. D54, 2328 (1996). 
[62] see e.g., S.F. King and P.L. White, Phys. Rev. D52, 4183 (1995) for a list of the MSSM RG equations.

[63] D. Pierce et al, Nucl. Phys. B491, 3 (1997).

[64] M. Drees and M. Nojiri, Nucl. Phys. B369, 54 (1992).

[65] K. Dienes, C. Kolda and J. March-Russell, Nucl. Phys. B492, 104 (1997).

[66] S. Dimopoulos, S. Thomas and J. Wells, Nucl. Phys. B488, 39 (1997).

[67] J. Feng and T. Moroi, Phys. Rev. D58, 035001 (1998).

[68] See, e.g., G. Kane, Nucl. Phys. Proc. Suppl. 62, 144 (1998) and references therein.

[69] A. Datta, M. Guchait and N. Parua, Phys. Lett. B395, 54 (1997).

[70] H. Murayama, hep-ph/9410285, Invited talk presented at the 22nd INS International Symposium on Physics with High Energy Colliders, Tokyo, Japan, March 8-10, 1994, appeared in Proceedings of INS Symposium, World Scientific, 1994.

[71] F. Gabbiani, E. Gabrielli, A. Masiero, and L. Silvestrini, Nucl. Phys. B477, $321(1996)$.

[72] M. Dine and A.E. Nelson, Phys. Rev. D48, 1277 (1993).

[73] M. Dine, A. E. Nelson, and Y. Shirman, Phys. Rev. D51, 1362 (1995). 
[74] M. Dine, A. E. Nelson, Y. Nir, and Y. Shirman, Phys. Rev. D53, 2658 (1996).

[75] C.D. Carone, H. Murayama, Phys. Rev. D53, 1658 (1996).

[76] S. Dimopoulos, M. Dine, S. Raby, and S. Thomas, Phys. Rev. Lett. 76, 3494 (1996); G. Dvali, G.F. Giudice, and A. Pomarol, Nucl. Phys. B478, 31 (1996); K.S. Babu, C. Kolda, and F. Wilczek, Phys. Rev. Lett. 77, 3070 (1996); A. Riotto, O. Tornkvist, and R.N. Mohapatra, Phys. Lett. B388, 599 (1996); S. Dimopoulos and G.F. Giudice, Phys. Lett. B393, 72 (1997); S.P. Martin, Phys. Rev. D55, 3177 (1997); S. Dimopoulos, S. Thomas, and J.D. Wells, Nucl. Phys. B488, 39 (1997); J.A. Bagger, K. Matchev, D.M. Pierce, and R.-J. Zhang, SLAC-PUB-7310, Phys. Rev. D55, 3188 (1997).

[77] S. Dimopoulos, G.F. Giudice, and A. Pomarol, Phys. Lett. B389, 37 (1996).

[78] D. Nemeschansky, Nucl. Phys. B234, 379 (1984).

[79] T. Moroi, H. Murayama, and M. Yamaguchi, Phys. Lett. B303, 289 (1993).

[80] I. Affleck and M. Dine, Nucl. Phys. B249, 361 (1985).

[81] D.H. Lyth and E.D. Stewart, Phys. Rev. Lett. 75, 201 (1995); Phys. Rev. D53, 1784 (1996).

[82] H. Pagels and J.R. Primack, Phys. Rev. Lett. 48223 (1982).

[83] P. Fayet, Phys. Lett. B175 471 (1986). 
[84] N. Arkani-Hamed, C.D. Carone, L.J. Hall, and H. Murayama, hepph/9607298, Phys. Rev. D54, 7032 (1996).

[85] I. Dasgupta, B.A. Dobrescu, and L. Randall, BUHEP-96-25, Nucl. Phys. B483, 95 (1997).

[86] L. Alvarez-Gaume, M. Claudson, and M.B. Wise, Nucl. Phys. B207, 96 (1982); M. Dine and W. Fischler, Phys. Lett. B110, 227 (1982).

[87] K.-I. Izawa and T. Yanagida, Prog. Theor. Phys. 95, 829 (1996).

[88] K. Intriligator and S. Thomas, Nucl. Phys. B473, 121 (1996).

[89] H. Murayama, H. Suzuki, T. Yanagida, and J. Yokoyama, Phys. Rev. D50, 2356 (1994).

[90] M.K. Gaillard, H. Murayama, and K.A. Olive, Phys. Lett. B355, 71 (1995).

[91] M. Dine, L. Randall, and S. Thomas, Nucl. Phys. B458, 291 (1996).

[92] A.D. Linde, Phys. Lett. 160B, 243 (1985).

[93] S. Davidson, H. Murayama, and K.A. Olive, Phys. Lett. B328, 354 (1994).

[94] V.A. Kuzmin, V.A. Rubakov, and M.E. Shaposhnikov, Phys. Lett. B191, 171 (1987); S.Yu. Khlebnikov and M.E. Shaposhnikov, Nucl. Phys. B308, 885 (1988).

[95] B.A. Campbell, S. Davidson, and K.A. Olive, Phys. Lett. B303, 63 (1993); Nucl. Phys: B399, 111 (1993). 
[96] H. Murayama and T. Yanagida, Phys. Lett. B322, 349 (1994).

[97] A.D. Dolgov and D.P. Kirilova, Yad. Fiz. 51, 273 (1990).

[98] B. de Carlos, J.A. Casas, F. Quevedo, and E. Roulet, Phys. Lett. B318, 447 (1993).

[99] G.D. Coughlan, R. Holman, P. Ramond, and G.G. Ross, Phys. Lett. B140, 44 (1984).

[100] T. Moroi, M. Yamaguchi, and T. Yanagida, Phys. Lett. B342, 105 (1995).

[101] T. Banks, D.B. Kaplan, and A.E. Nelson, Phys. Rev. D49, 779 (1994).

[102] M. Kawasaki and T. Moroi, Astrophys. J. 452506 (1995).

[103] L. Randall and S. Thomas, Nucl. Phys. B449, 229 (1995).

[104] M. Kawasaki, T. Moroi and T. Yanagida, Phys. Lett. B370 52 (1996).

[105] K. Agashe, N. Arkani-Hamed, and M. Graesser, private communication.

[106] S. Dimopoulos and S. Raby, Nucl. Phys. B192, 353 (1981).

[107] E. Witten, Nucl. Phys. B188, 513 (1981).

[108] M. Dine, W. Fischler, and M. Srednicki, Nucl. Phys. B189, 575 (1981).

[109] K. Inoue, A. Kakuto, H. Komatsu and S. Takeshita, Prog. Theor. Phys. 68, 927 (1982); Prog. Theor. Phys. 71, 413 (1984); L.E. Ibáñez, Phys. 
Lett. B118, 73 (1982); Nucl. Phys. B218, 514 (1983); L. Alvarez-Gaumé, J. Polchinski and M. Wise Nucl. Phys. B221, 495 (1983).

[110] P. Abreu et al., Eur. Phys. J. C1, 1 (1998); K. Ackerstaff et al., Eur. Phys. J. C2, 213 (1998); R. Barate et al., Eur. Phys. J. C2, 417 (1998); M. Acciari et al., Eur. Phys. J. C4, 207 (1998).

[111] M. Dine and A.E. Nelson, Phys. Rev.'D48, 1277 (1993).

[112] M. Dine, A.E. Nelson and Y. Shirman, Phys. Rev. D51, 1362 (1995).

[113] M. Dine, A.E. Nelson, Y. Nir and Y. Shirman, Phys. Rev. D53, 2658 (1996).

[114] K. Intriligator, N. Seiberg and S. Shenker, Phys. Lett. B342 152 (1995); H. Murayama, Phys. Lett. B355, 187 (1995); E. Poppitz and S. P. Trivedi, Phys. Lett. B365, 125 (1996); K.-I. Izawa and T. Yanagida, Prog. Theor. Phys. 95, 829 (1996); K. Intriligator and S. Thomas, Nucl. Phys. B473, 121 (1996); C. Csaki, L. Randall, and W. Skiba, Nucl. Phys. B479, 65 (1996); E. Poppitz, Y. Shadmi, and S.P. Trivedi, Nucl. Phys. B480, 125 (1996); C.-L. Chou, Phys. Lett. B391, 329 (1997); T. Hotta, K.-I. Izawa, and T. Yanagida, Phys. Rev. D55, 415 (1997); E. Poppitz, Y. Shadmi, and S. P. Trivedi, Phys. Lett. B388, 561 (1996); C. Csaki, L. Randall, W. Skiba, and R. G. Leigh, Phys. Lett. B387 791 (1996); K. Intriligator and S. Thomas, SLAC-PUB-7143; E. Poppitz and S.P. Trivedi, Phys. Rev. D55, 5508 (1997); C. Csaki, M. Schmaltz, and W. Skiba, Phys. Rev. D55, 
7840 (1997); L. Randall, Nucl. Phys. B495, 37 (1997); N. Haba, N. Maru, and T. Matsuoka, Nucl. Phys. B497, 31 (1997); N. Arkani-Hamed, J. MarchRussell, and H. Murayama, Nucl. Phys. B509, 3 (1998); E. Poppitz, and S.P. Trivedi, Phys. Lett. B401, 38 (1997); N. Haba, N. Maru, and T. Matsuoka, Phys. Rev. D56, 4207 (1997); Y. Shadmi, Phys. Lett. B405, 99 (1997); R. G. Leigh, L. Randall, and R. Rattazzi, Nucl. Phys., B501, 375 (1997); K.-I. Izawa, Y. Nomura, K. Tobe, and T. Yanagida, Phys. Rev. D56, 2886 (1997); C. Csaki, L. Randall, and W. Skiba, Phys. Rev. D57, 383 (1998); Y. Nomura, K. Tobe, and T. Yanagida, Phys. Lett. B425, 107 (1998).

[115] H. Murayama, Phys. Rev. Lett. 79, 18 (1997).

[116] S. Dimopoulos, G. Dvali, R. Rattazzi, and G.F. Giudice, Nucl. Phys. B510, $12(1998)$.

[117] M. Luty, Phys. Lett. B414, 71 (1997); M. Luty and J. Terning, Phys. Rev. D57, 6799 (1998); Y. Shirman, PUPT-1721, Phys. Lett. B417, 281 (1998).

[118] K. Intriligator and N. Seiberg, Nucl. Phys. Proc. Suppl. 45BC, 1 (1996).

[119] C. Nappi and B. Ovrut, Phys. Lett. B113, 175 (1982); M. Dine and W. Fischler, Nucl. Phys. B204, 346 (1982); L. Alvarez-Gaumé, M. Claudson and M. Wise, Nucl. Phys. B207 96 (1982). 
[120] J. Ellis, J.F. Gunion, H. E. Haber, L. Roszkowski, and F. Zwirner, Phys. Rev. D39, 844 (1989), and references therein.

[121] R. Barbieri, S. Ferrara, and C. A. Savoy, Phys. Lett. 119B, 343 (1982); A. H. Chamseddine, R. Arnowitt, and P. Nath, Phys. Rev. Lett. 49, 970 (1982); L. J. Hall, J. Lykken and S. Weiberg, Phys. Rev. D27, 2359 (1983).

[122] J. Polonyi, Budapest preprint, KFKI-93 (1977).

[123] P. Nilles, Phys. Lett. 115B, 193 (1982); Nucl. Phys. B217, 366 (1983).

[124] L. O'Rafeartaigh, Nucl. Phys. B96, 331 (1975).

[125] G.F. Giudice and A. Massiero, Phys. Lett. B206, 480 (1988).

[126] S. Dimopoulous and H. Georgi, Nucl. Phys. B193, 150 (1981).

[127] Y. Nir and N. Seiberg, Phys. Lett. B309, 337 (1993).

[128] P. Pouliot and N. Seiberg, Phys. Lett. B318, 169 (1993); D. B. Kaplan and M. Schmaltz, Phys. Rev. D49, 3741 (1994); L. J. Hall and H. Murayama, Phys. Rev. Lett., 75, 3985 (1995); C. D. Carone, L. J. Hall, and H. Murayama, Phys. Rev. D53, 6282 (1996); ibid., D54, 2328 (1996); R. Barbieri, G. Dvali, and L. J. Hall, Phys. Lett. B377, 76 (1996); R. Barbieri, L. J. Hall, S. Raby, and A. Romanino, Nucl. Phys. B493, 3 (1997); R. Barbieri, L. J. Hall, A. Romanino, Phys. Lett. B401, 47 (1997); P. H. Frampton and O. C. W. Kong, Phys. Rev. D55, 5501 (1997); Phys. Rev. Lett. 77, 1699 (1996). 
[129] M. Leurer, Y. Nir, and N. Seiberg, Nucl. Phys. B420 468, (1994).

[130] Y. Nir, Phys. Lett. B354 107, (1995); Y. Nir and R. Rattazzi, Phys. Lett. B382, 363 (1996).

[131] V. S. Kaplunovsky and J. Louis, Phys. Lett. B306, 269 (1993).

[132] N. Arkani-Hamed, C. D. Carone, L. J. Hall, and H. Murayama, Phys. Rev. D54, 7032 (1996); I. Dasgupta, B. A. Dobrescu, and L. Randall, Nucl. Phys. B483, 95 (1997).

[133] G. Dvali, G.F. Giudice, and A. Pomarol, Nucl. Phys. B478 31, (1996).

[134] E. Witten, Phys. Lett. 105B, 267 (1981).

[135] D. Nemeschansky, Nucl. Phys. B234, 379 (1984).

[136] P. Ciafaloni and A. Pomarol, Phys. Lett. B404, 83 (1997).

[137] K. Agashe and M. Graesser, Nucl. Phys. B507, 3 (1997).

[138] G.F. Giudice and R. Rattazzi, CERN-TH-97-145, Nucl. Phys. B511, 25 (1998).

[139] S. Dimopoulos, G. Dvali and R. Rattazzi, CERN-TH/97-186, Phys. Lett. B413, 336 (1997).

[140] T. Yanagida, Phys. Lett. B400 109, (1997).

[141] P. Nilles and N. Polonsky, Phys. Lett. B412, 69 (1997). 
[142] R. Rattazzi and U. Sarid, Nucl. Phys. B501, 297 (1997); F.M. Borzumati, WIS-96/50/Dec.-PH, hep-ph/9702307.

[143] R. Barbieri and G.F. Giudice, Nucl. Phys. B306 63 (1989).

[144] P. Janot, talk presented at E.P.S. HEP Conference, Jerusalem (1997); S. Assay, talk presented at E.P.S. HEP Conference, Jerusalem (1997); D. Buskulic et al., Phys. Lett. B373, 246 (1996).

[145] K.R. Dienes, C. Kolda, and J. March-Russell, Nucl. Phys. B492, 104 (1997); S. Dimopoulos, S. Thomas, and J.D. Wells, Nucl. Phys. B488, 39 (1997).

[146] T. Elliott, S.F. King, and P.L. White, Phys. Lett. B351, 213 (1995); S.F. King and P.L. White, Phys. Rev. D52, 4183 (1995).

[147] G. Anderson and D. Castaño, Phys. Lett. B347, 300 (1995). 